\title{
On the Uniqueness of Solution to the Cauchy Problem for Elliptic Equations in Two Variables
}

Dedicated to Professor Shigetake Matsuura on the sixtieth anniversary of his birthday

By

Shin-ichi FUJII*

\section{Contents}

§1. Introduction

$\S 2$. Reduction of the proof of Theorem

$\S 3$. Factorization of some third degree polynomial

$\S 4$. Pseudodifferential operators used in the proof of Proposition 2.3

$\S 5$. The basic estimates

$\S 6$. Lemmas on commutation

$\S 7 . \quad$ Lemmas on estimates

\$8. The derivation of (2.13) and (2.14)

§. Proof of Proposition 2.3

§10. Appendix. Proof of Lemma 4.7

References

\section{§1. Introduction}

The famous example of Plis ([11] Theorem 4) has shown us that the uniqueness in the Cauchy problem for four-fold characteristics does not hold in general even in smooth coefficients and two independent variables. Similar but more general examples of two independent variables have been constructed by Nakane [7]. But when we consider triple characteristics case the results are not ap-

Communicated by S. Matsuura, April 12, 1938.

* Research Institute for Mathematical Sciences, Kyoto University, Kyoto 606, Japan. 
plicable and we have few of information. There exists only a counter example with non-smooth coefficients. (See [3]).

In this paper we shall give a positive result for the uniqueness in case of equations with smooth coefficients of two independent variables and with triple characteristics, in spite of the existence of such a counterexample. This result will show us the uniqueness almost always holds in such a case.

In two dimensional case, there is an effective reduction technique to a simple case that the set, on which the multiplicity of characteristic roots vary coincides with the initial surface. This will work well in our proof. (See [13], [14], [15] for other related results.) Our main result is the following.

Theorem. Let $P$ be a linear elliptic operator of order $m(m \geq 1)$ with $C^{\infty}$ coefficients in an open neighbourhood $\omega$ of the origin in $R^{2}$. Under the following assumptions (a), (b) one can find an open neighbourhood $\omega^{\prime} \subset \omega$ of the origin such that every $u \in C^{\infty}(\omega)$ satisfying $P u=0$ and $\left.u\right|_{t \leq 0}=0$ vanishes in $\omega^{\prime}$.

Assumptions. We denote by $P_{m}(t, x, \tau, \xi)$ the principal symbol.

(a) $p_{m}(t, x, \tau, \xi)=\prod_{i=1}^{3} \prod_{j=0}^{k_{i}} p_{i j}(t, x, \tau, \xi)$, where $k_{i} \geq 0, p_{i j}(t, x, \tau, \xi)=(\tau-$ $\left.a_{i j} \xi\right)^{i}+\sum_{l=0}^{i-1} a_{i j l}(t, x) \xi^{i-l}\left(\tau-a_{i j} \xi\right)^{l}$ for $j \neq 0$, and $p_{i 0}=1$, with distinct non real constants $a_{i j}$ and $a_{i j l} \in C^{\infty}(\omega)$ satisfying $a_{i j l}(0,0)=0$.

(b) Let $\Delta_{i j}(t, x), i \neq 1, j \neq 0$ (resp. $\Delta_{j}^{\prime}(t, x), j \neq 0$ ) is the discriminant of $p_{i j}(t, x, \tau, 1)$ (resp. $\partial_{\tau} p_{3 j}(t, x, \tau, 1)$ ) as a polynomial in $\tau$. We assume that $\Delta_{i j}(t, x)\left(\right.$ resp. $\left.\Delta_{j}^{\prime}(t, x)\right)$ has a finite order zero at $(0,0)$ unless $\Delta_{i j} \equiv 0$ (resp. $\left.\Delta_{j}^{\prime} \equiv 0\right)$ in $\omega$, where we say that $g \in C^{\infty}(\omega)$ has a finite order zero at $(0,0)$ when $g(0,0)=0$ and $\partial_{t, x}^{\infty} g(0,0) \neq 0$ for some $\alpha$.

We shall mention the organization of this paper. In section two we carry out a reduction as above and the proof of the main theorem is reduced to that of Proposition 2.3. We devote ourselves to prove this from section three to section ten. The essential part of proof is section three and section eight. The idea of proof is a factorization of the symbol of the operator modulo terms of order less than its order minus one, which is the same as in papers Matsumoto [6], Ōkaji [9]. Another sections except for section nine is those for tedious technicalities. The argument in section nine is routine one.

Remark on notations. The set of all positive integers and all non-negative integers are denoted by $\boldsymbol{N}$ and $\boldsymbol{Z}_{+}$respectively. 


\section{Acknowledgement}

The author thanks Professor S. Matsuura, Professor N. Iwasaki, and Professor K. Nishiwada for helpful advice and comments.

\section{§2. Reduction of the Proof of Theorem}

Reduction mentioned above works well also in our case by virtue of Watanabe's theorem in [12].

First of all, it is easy to see that by making a linear change of coordinates which preserves $x$-axis one can reduce (b) to a special case that $\Delta_{i j}(t, 0)$ (resp. $\left.\Delta_{j}^{\prime}(t, 0)\right)$ has a finite order zero at $t=0$ (namely, $\Delta_{i j}(0,0)=0$ and $\left(\partial_{t}^{k_{i j}} \Delta_{i j}\right)(0,0)$ $\neq 0$ for some $k_{i j}$ for example) unless $\Delta_{i j} \equiv 0$ (resp. $\Delta_{j}^{\prime} \equiv 0$ ) in $\omega$, since (b) is invariant under such change of coordinates. Indeed, we note that for $a(t, x) \in C^{\infty}$ near the origin in $\boldsymbol{R}^{2}$ and $c \in \boldsymbol{R}$,

$$
\left.\partial_{t}^{j}(a(t, x+c t))\right|_{(0,0)}=\sum_{k=0}^{j}\left(\begin{array}{l}
j \\
k
\end{array}\right)\left(\partial_{t}^{j-k} \partial_{x}^{k} a\right)(0,0) c^{k} .
$$

By the change of coordinates $(t, x) \rightarrow\left(t^{\prime}, x^{\prime}\right)=(t, x-c t)$, the principal symbol of $P$ is transformed to $\prod_{i=1}^{3} \prod_{j=0}^{k_{i}} \tilde{p}_{i j}$ where $\tilde{p}_{i j}=p_{i j}\left(t^{\prime}, x^{\prime}+c t^{\prime}, \tau^{\prime}+c \xi^{\prime}, \xi^{\prime}\right)$ so that (a) clearly holds for $\tilde{p}_{i j}$ and the discriminant of $\tilde{p}_{i j}\left(t, x, \tau^{\prime}, 1\right)$ and $\partial_{\tau} \tilde{p}_{3 j}\left(t, x, \tau^{\prime}, 1\right)$ are $\Delta_{i j}\left(t^{\prime}, x^{\prime}+c t^{\prime}\right)$ and $\Delta_{j}^{\prime}\left(t^{\prime}, x^{\prime}+c t^{\prime}\right)$ respectively. Therefore the above special case of (b) holds for a suitable choise of $c$ by virtue of (b). So we may assume this instead of (b). Set $\Lambda=\left\{(i, j) ; \Delta_{i j} \neq 0\right\} \cup\left\{j ; \Delta_{j}^{\prime} \neq 0\right\} . \quad \Lambda=\phi$ means that the number of distinct characteristic roots is constant in $\omega$ and at most three. So our theorem immediately follows from Watanabe's theorem in [9] when $\Lambda=\phi$. Thus we may assume $\Lambda \neq \phi$. We will prove Theorem by contradiction when $\Lambda \neq \phi$. Suppose that the assertion of Theorem is not true. By Malgrange's preperation theorem one can find $T>0, X>0$ such that $\omega_{1} \equiv(-T, T) \times(-X, X)$ $\subset \omega$, and that for $(t, x) \in \omega_{1}$,

$$
\begin{aligned}
& \Delta_{i j}(t, x)=b_{i j}(t, x) r_{i j}(t, x), \quad(i, j) \in \Lambda, \\
& \Delta_{j}^{\prime}(t, x)=b_{j}^{\prime}(t, x) r_{j}^{\prime}(t, x), \quad j \in \Lambda, \\
& \Delta(t, x) \equiv \prod_{(i, j) \in \Lambda} \Delta_{i j}(t, x) \prod_{j \in \Lambda} \Delta_{j}^{\prime}(t, x)=b(t, x) r(t, x), \\
& r_{i j}(t, x)=t^{k_{i j}}+\sum_{i=0}^{k_{i j}-1} d_{i j l}(x) t^{l}, \\
& r_{j}^{\prime}(t, x)=t^{k_{j}^{\prime}}+\sum_{l=0}^{k_{j}^{\prime}-1} \tilde{d}_{i l}(x) t^{l}, \\
& r(t, x)=t^{k}+\sum_{l=0}^{k-1} d_{l}(x) t^{l},
\end{aligned}
$$

where $b_{i j}, b_{j}^{\prime}, b \in C^{\infty}\left(\omega_{1}\right), d_{i j l}, \tilde{d}_{j l}, d_{l} \in C^{\infty}((-X, X))$ satisfying that $b_{i j}(t, x) \neq 0$, 
$b_{j}^{\prime}(t, x) \neq 0, b(t, x) \neq 0$ for any $(t, x) \in \omega_{1}$, and that $d_{i j l}(0)=0, \tilde{d}_{j l}(0)=0, d_{l}(0)=0$, and $k_{i j}, k_{j}^{\prime}, k$ are positive integers. We may assume that $r(z, x) \neq 0$ when $z \in C_{\text {, }}$ $|z| \geq T / 2$, since $d_{l}(0)=0$. We set

$$
\begin{aligned}
& J_{i}=\{x \in(-X, X) ; \#(\{z \in C ; r(z, x)=0\}) \geq i\}, i=1, \cdots, k ; \\
& J_{k}^{\prime}=J_{k} ; J_{i}^{\prime}=J_{i} \backslash \operatorname{cl}\left(J_{i+1}\right), i=1, \cdots, k-1,
\end{aligned}
$$

where $\mathrm{cl}\left(J_{i}\right)$ denotes the closure of $J_{i}$ in $(-X, X)$. Then $J_{i}^{\prime}$ are open in $(-X, X)$, $\cup_{i} J_{i}^{\prime}$ is dense in $(-X, X)$, and $\#(\{z \in C ; r(t, x)=0\})=i$ for $x \in J_{i}^{\prime}$. Next we set

$$
J_{i, j}=\left\{x \in J_{i}^{\prime} ; \text { 莽 }(\{z \in \boldsymbol{C} \backslash \boldsymbol{R} ; r(t, x)=0\}) \geq j\right\}, j=1, \cdots, i ; J_{i, i}^{\prime}=J_{i, i} ;,
$$

$J_{i, j}^{\prime}=J_{i, j} \backslash \operatorname{cl}\left(J_{i, j+1}\right), j=0, \cdots, i-1$, where $\operatorname{cl}\left(J_{i, j+1}\right)$ is the closure of $J_{i, j+1}$ in $(-X, X)$. Then $J_{i, j}^{\prime}$ are open in $(-X, X), \cup_{i} \cup_{j} J_{i, j}^{\prime}$ is dense in $-(X, X)$, and $\#(\{z \in \boldsymbol{C} \backslash \boldsymbol{R} ; r(z, x)=0\})=j$ for $x \in J_{i, j}^{\prime}$. By the assumption one can find $u \in C^{\infty}(\omega)$ satisfying $P u=0$ in $\omega,\left.u\right|_{t \leq 0}=0$, and that supp $u \cap \omega_{1} \neq \phi$. So there exists $J_{i, j}^{\prime}$ and an open interval $I$ such that $I \subset J_{i, j}^{\prime}$ and $((-T, T) \times I) \cap$ supp $u \neq \phi$. Since both of the numbers of distinct roots and distinct non real roots of the equation in $\sigma, r(t, x)=0$, are constant when $x \in I$, it follows that for $i>j$, there exists $\theta_{l} \in C^{\infty}(I), l=1, \cdots, i-j$ with $-T / 2<\theta_{1}(x)<\cdots<\theta_{i-j}(x)$ $<T / 2$, positive integers $r_{l}, l=1, \cdots, i$, and $\tilde{b} \in C^{\infty}((-T, T) \times I)$ with $b(t, x) \neq 0$ for all $(t, x)$ such that $\Delta(t, x)=\tilde{b}(t, x) \prod_{l=1}^{i_{1}}\left(t-\theta_{1}(x)\right)^{r_{l}}$, and that if $i=j, \Delta(t, x) \neq 0$ for all $(t, x) \in(-T, T) \times I$. We set $i_{1}=i-j, \theta_{0}=-T, \theta_{i_{1}+1}=T$, and $A_{l}=\{(t, x) \in$ $\left.[-T, T] \times I ; \theta_{l}(x) \leq t<\theta_{l+1}(x)\right\}, l=0, \cdots, i_{1}$. We can take $i_{0}=\inf \left\{i ; A_{i} \cap\right.$ supp $u \neq \phi\}$ and two cases arise, in one supp $u \cap\left\{t=\theta_{i_{0}}(x)\right\}=\phi$, in the other supp $u \cap\left\{t=\theta_{i_{0}}(x)\right\} \neq \phi$.

In the former case we take $\left(t_{0}, x_{0}\right) \in A_{i_{0}} \cap \operatorname{supp} u$ and $\varepsilon>0$ so that $\left[x_{0}-\varepsilon, x_{0}+\varepsilon\right] \subset I$. Consider paraboles $\mathscr{Q}_{c}: \psi_{c}=t+\varepsilon^{-2}\left(x-x_{0}\right)^{2}(c+T)-c=0$. We can take $\varepsilon$ so small that

$$
\left\{(t, x) \in[-T, T] \times I ; \psi_{t_{0}}=0, t=b_{i_{0}+1}(x)\right\}=\phi .
$$

We can take

$$
\begin{aligned}
& c_{0}=\inf \left\{c ; \operatorname{supp} u \cap \mathscr{P}_{c} \neq \phi\right\}\left(t_{0} \geq c_{0} \geq 0\right) \quad \text { and } \\
& \left(t_{1}, x_{1}\right) \in \operatorname{supp} u \cap \mathscr{Q}_{c} \cap A_{i_{0}} .
\end{aligned}
$$

Then $\Delta\left(t_{1}, x_{1}\right) \neq 0$ implies that the multiplicities of roots of the equation $p_{m}(t, x, \tau, 1)=0$ are constant near the point $\left(t_{1}, x_{1}\right)$. Since in this case by virtue of the fact that the dimension is two, the multiplicities of roots of the charac- 
teristic equation of $P$ in $\sigma$ defined for $\eta, N \in \boldsymbol{R}^{2} \backslash(0)$ with $\langle N, \eta\rangle=0$, where $\langle$,$\rangle is the standard inner product in \boldsymbol{R}^{2}, p_{m}(t, x, \sigma N+\eta)=0$, are constant when $N, \eta$ are fixed and $(t, x)$ is close to $\left(t_{1}, x_{1}\right)$ and since $u(t, x)=0$ when $\psi_{c_{0}}(t, x) \leq 0$, after the suitable linear change of coordinates at $\left(t_{1}, x_{1}\right)$ by an orthogonal matrix Watanabe's theorem can be applied to conclude that $\left(t_{1}, x_{1}\right) \notin$ supp $u$ which is contradiction.

In the latter case there exists $\left(t_{1}, x_{1}\right) \in \operatorname{supp} u \cap\left\{t=\theta_{i_{0}}(x)\right\}$. Since the number of distinct roots of the equation $r(t, x)=0$ in $t$ is constant in $x \in I$, any of $\Delta_{i j}$ and $\Delta_{j}^{\prime}$ is of the form $\left(t-\theta_{i_{0}}(x)\right)^{r} c(t, x)$ with a positive integer $r$ and a smooth $c$ satisfying $c\left(t_{1}, x_{1}\right) \neq 0$, unless it is identically zero or does not vanish at $\left(t_{1}, x_{1}\right)$. We need a lemma.

Lemma 2.1. Suppose that the discriminant of a polynomial in $\sigma$,

$$
\begin{aligned}
& q(\sigma, s, y)=\sigma^{l}+a_{1}(s, y) \sigma^{l-1}+\cdots+a_{l}(s, y), \\
& a_{i} \in C^{\infty}(U), U=[-S, S] \times[-Y, Y]
\end{aligned}
$$

is equal to $s^{n} D(s, y)$ with a positive integer $n$ and $D \in C^{\infty}(U)$ satisfying $\inf _{U}$ $|D(s, y)|>0$. Then the roots of the equation in $\sigma, q(\sigma, s, y)=0$, is expressed by $l$ continuous functions $\mu_{j}(s, y), j=1, \cdots, l$ for $(s, y) \in\left[0, S_{1}\right) \times(-Y, Y)$ with a $S_{1} \in(0, \min (S, 1))$, satisfying there exists a positive integer $h$ such that $\mu_{j}\left(s^{h}, y\right)$ belongs to $C^{\infty}\left(\left[0, S_{1}\right) \times(-Y, Y)\right)$ for all $j$.

Proof. Set

$$
a_{i}^{\prime}(z, y, w)=\sum_{j<N}(j !)^{-1} z^{j}\left(\partial_{s}^{j} a_{i}\right)(0, y)+((N-1) !)^{-1} z^{N} w_{i}, i=1, \cdots, l \text { for }
$$
$z \in \boldsymbol{C}, w=\left(w_{1}, \cdots, w_{l}\right) \in \boldsymbol{C}^{l}$, and

$$
\begin{aligned}
\tilde{a}_{i}(s, y) & =\int_{0}^{1}\left(\partial_{s}^{N} a_{i}\right)(\theta s, y)(1-\theta)^{N-1} d \theta, \\
q^{\prime}(\sigma, z, y, w) & =\sigma^{l}+\sum_{i<l} a_{i}^{\prime}(z, y, w) \sigma^{i} .
\end{aligned}
$$

Since $a_{i}(s, y)-a_{i}^{\prime}(s, y, w)=s^{N}\left(\tilde{a}_{i}(s, y)-w_{i}\right) /(N-1)$ !, for any $N>n$ there exists $S^{\prime} \in(0, \min (1, S))$ such that when $|z| \leq S^{\prime},|w| \leq M \equiv \max _{i} \sup _{U}\left(\left|\tilde{a}_{i}\right|+1\right)$, the discriminant of polynomial in $\sigma$ is equal to $z^{n} D^{\prime}(z, y, w)$ where $D^{\prime}$ is a polynomial in $(z, w)$ with coefficients in $C^{\infty}([-Y, Y])$ satisfying inf $\left\{\left|D^{\prime}(z, y, w)\right|\right.$; $\left.|z| \leq S^{\prime},|y| \leq Y,|w| \leq M\right\}>0$. We set

$$
\begin{aligned}
\Gamma=\Gamma_{a, b}= & \left\{(z, y, w) ;|z|<S^{\prime},|y|<Y,|w|<M, z \in\left\{\mathrm{re}^{v-1} \theta\right.\right. \\
& \left.\left.0<r<S^{\prime}, a<\theta<b\right\}\right\} \text { for } a, b \in \boldsymbol{R} \text { such that } b-a<\pi .
\end{aligned}
$$

Since $\Gamma$ is simply connected, the implicit function theorem and the monodromy 
theorem give $\mu_{j, \Gamma}(z, y, w) \in C^{\infty}(\Gamma), j=1, \cdots, l$ which are holomorphic in $(z, w)$ and satisfy $q^{\prime}\left(\mu_{j, \Gamma}(z, y, w), z, y, w\right)=0$ and $\mu_{i, \Gamma}(z, y, w) \neq \mu_{j, \Gamma}(z, y, w)$ if $i \neq j$, for all $(z, y, w) \in \Gamma$. Set $\Phi_{\Gamma}=\left\{\mu_{j, \Gamma} ; j=1, \cdots, l\right\}, R=\{(z, y, w)$; $\left.0<|z|<S^{\prime},|y|<Y,|w|<M\right\}, \mathcal{U}=\left\{\Gamma_{a, b} ; a, b\right\}$. Let $R^{*}$ be the set whose points are determined by a pair $(p, \varphi), p \in R, \varphi \in \Phi_{\Gamma}$ such that $p \in \Gamma, \Gamma \in \mathcal{U}$ and two pairs $\left(p_{1}, \varphi_{1}\right),\left(p_{2}, \varphi_{2}\right)$ are identified if and only if $p_{1}=p_{2}$ and $\varphi_{1}=\varphi_{2}$ in a neighbourhood of $p_{1}$. We define the topology of $R^{*}$ so that the fundamental neighbourhood system of $\left(p_{0}, \varphi_{0}\right) \in R^{*}$ with $p_{0} \in \Gamma$ and $\varphi_{0} \in \Phi_{\Gamma}$ is a family of sets $\{(p, \varphi) ; p \in V\}$ such that there exists $\Gamma_{1} \in \mathcal{U}$ such that $V \subset \Gamma_{1}, \varphi \in \Phi_{\Gamma_{1}}$, and such that $\varphi=\varphi_{0}$ in a neighbourhood of $p_{0}$ and $V$ is a open set with $p_{0} \in V$. This definition is well defined. We define a mapping $\pi: R^{*} \rightarrow R$ by $\pi\left(p^{*}\right)=p$ if $p^{*}=(p, \varphi)$. Then a pair $\left(R^{*}, \pi\right)$ is a covering space of $R$. (For the definition of the covering space we refer L. S. Pontryagin: Topological Groups. See also Ahlfors Sario Riemann Surfaces.) Since $R$ is arcwise connected, each component of $R^{*}$ with the restriction of $\pi$ to it is a arcwise connected covering space of $R$. It is clear that the set $V_{\varphi, \Gamma}^{*}=\{(p, \varphi) ; p \in \Gamma\}$ is connected for any $\Gamma \in \mathcal{U}$ and $\varphi \in \Phi_{\Gamma}$. Let $h_{i} \in \mathbb{Z}$ be the number of sheets of a covering space $\left(R_{i}^{*},\left.\pi\right|_{R_{i}^{*}}\right)$ of $R$ where $R_{i}^{*}$ is the component of $R^{*}$ containing $V_{\varphi, \Gamma}^{*}$ with $\Gamma=\Gamma_{-\pi / 4, \pi / 4}, \varphi=\mu_{i, \Gamma}$. We can construct in the same way as above a covering space $\left(R_{i 1}^{*}, \pi_{i}\right)$ of $R$ for any $i=1, \cdots, l$ by the family of functions $\mu_{i, j, \Gamma}(z, y, w)$ $=\mu_{j, \Gamma_{i}}\left(z^{h_{i}}, y, w\right), j=1, \cdots, l$ where $\Gamma=\Gamma_{a, b} \in \mathcal{Q}$ with $b-a<\pi / h_{i}$ and $\Gamma_{i}=$ $\left\{\left(z^{h_{i}}, y, w\right) ;(z, y, w) \in \Gamma\right\}$. Then since any closed curve in $R$ is homotopic to one of curves $C_{m}: t \rightarrow\left(S^{\prime} / 2 e^{2 \pi v-1} m t, 0,0\right), t \in[0,1] ; m \in \mathbb{Z}$, for any open interval is simply connected, the number of sheets of a covering space $\left(R_{i 2}^{*},\left.\pi_{i}\right|_{R_{i 2}^{*}}\right)$ of $R$ where $R_{i 2}^{*}$ is the component of $R_{i 1}^{*}$ containing a connected subset of $\{(p, \varphi)$; $\left.\varphi \in \Gamma_{0}\right\}$ with $\Gamma_{0}=\Gamma_{-\pi /\left(4 h_{i}\right), \pi /\left(4 h_{i}\right)}$ and $\varphi=\mu_{i, i, \Gamma}$, is one. So, one can find $F_{i} \in$ $C^{\infty}(R)$ which is holomorphic in $(z, w)$ satisfying $F_{i}=\mu_{i, i, \Gamma}$ on $\Gamma_{0}$ and can be extended to a function $\widetilde{F}$ in $C^{\infty}\left(\left\{(z, y, w) ;|z|<S^{\prime},|y|<Y,|w|<M\right\}\right)$ by Cauchy's integral formula. Therefore, $\mu_{i, \Gamma}\left(s^{h_{i}}, y, w\right)$ is $C^{\infty}$ in $\left[0, S^{\prime}\right) \times(-Y, Y)$ $\times\{|w|<M\}$ for $\Gamma=\Gamma_{-\pi / 4, \pi / 4}$. We will take $h=\prod_{i=1}^{l} h_{i}, S_{1}=S^{\prime}$, and with $\Gamma=\Gamma_{-\pi / 4, \pi / 4}, \mu_{i}(s, y)=\mu_{i, \Gamma}\left(s, y,\left(\tilde{a}_{i}(s, y)\right)\right)$ when $(s, y) \in\left(0, S_{1}\right) \times(-Y, Y)$, $=\widetilde{F}\left(0, y,\left(\tilde{a}_{i}(0, y)\right)\right)$ when $s=0$ and $y \in(-Y, Y)$. Then, these have required properties. The proof is complete.

We need a definition.

Definition 2.2. Let $U_{a, b}=[0, a]_{t} \times[-b, b]_{x}$ for $a>0, b>0$, and $h$ be a positive integer. We set 


$$
C_{h}^{\infty}\left(U_{a, b}\right)=\left\{g ; g \in C\left(U_{a, b}\right), g\left(t^{h}, x\right) \in C^{\infty}\left(U_{a^{1 / h}, b}\right)\right\} .
$$

Let $\varphi \in C^{\infty}([-b, b])$ be a non negative function with $\varphi^{1 / h} \in C^{\infty}([-b, b])$. We set

$$
\begin{aligned}
& F_{h, \varphi}\left(U_{a, b}\right) \text { (or simply } F_{h, \varphi} \text { if not confused) } \\
& =\left\{g ; g \in C_{h}^{\infty}\left(U_{a, b}\right) \text { with } g \equiv 0 \text {, or } g=(t \varphi)^{e} \tilde{g}, e h \in Z, e>0,\right. \\
& \left.\quad \tilde{g} \in C^{\infty}\left(U_{a, b}\right) \text { with } \inf _{\sigma_{a, b}}|\tilde{g}|>0\right\}, \\
& F_{h, \varphi}^{*}\left(U_{a, b}\right)=\left\{g \in F_{h, \varphi}, g \neq 0\right\} .
\end{aligned}
$$

We say for a function $g$ defined in a neighbourhood of $(0,0)$ in $[0, \infty)_{t} \times \boldsymbol{R}_{x}$ that $g$ is $C_{h}^{\infty}$ (resp. $F_{h, \varphi}, F_{h, \varphi}^{*}$ ) near $(0,0)$ if $g$ is defined in $U_{a, b}$ for some $a, b$ and $g \in C_{h}^{\infty}\left(U_{a, b}\right)$ (resp. $\left.F_{h, \varphi}\left(U_{a, b}\right), F_{h, \varphi}^{*}\left(U_{a, b}\right)\right)$.

We set $q_{i j}(t, x, \tau)=p_{i j}\left(t+\theta_{i_{0}}\left(x+x_{1}\right), x+x_{1}, \tau, 1\right), \tilde{\Delta}_{i j}(t, x)=\Delta_{i j}\left(t+\theta_{i_{0}}\left(x+x_{1}\right)\right.$, $\left.x+x_{1}\right)$, and $\widetilde{\Delta}_{j}^{\prime}(t, x)=\Delta_{j}^{\prime}\left(t+\theta_{i_{0}}\left(x+x_{1}\right), x+x_{1}\right)$, for $x \in\left(-\varepsilon_{0}, \varepsilon_{0}\right)$ with a small $\varepsilon_{0}$. We will factorize $q_{i j}$ when $(t, x)$ close to $(0,0)$ so that for some $h=h_{i j}$,

$$
\begin{aligned}
& q_{i j}(t, x, \tau)=\prod_{k=1}^{i}\left(\tau-\mu_{i j k}(t, x)\right), \mu_{i j k} \in C_{h}^{\infty} \quad \text { near the origin, and } \\
& \mu_{i j k}-\mu_{i j k^{\prime}} \in F_{h, 1} \quad \text { if } \quad \mu_{i j k}(0,0)-\mu_{i j k^{\prime}}(0,0)=0 .
\end{aligned}
$$

It is clear that $q_{2 j}$ will cause no problem because of the remark above Lemma 1. We want to consider $q_{3 j}$. When $\tilde{\Delta}_{3 j}(0,0) \neq 0$, there is also no problem. When $\widetilde{\Delta}_{j}^{\prime}(0,0) \neq 0$ and $\tilde{\Delta}_{3 j}(0,0)=0$,

$$
q_{3 j}(t, x, \tau)=\prod_{k=1}^{2} q_{j}^{(k)}(t, x, \tau) \quad \text { when }(t, x) \text { is close to }(0,0)
$$

so that $q_{j}^{(k)}$ is a polynomial in $\tau$ of degree $k$ with $C^{\infty}$ coefficients and the resultant of $q_{j}^{(1)}(0,0, \tau)$ and $q_{j}^{(2)}(0,0, \tau)$ is not zero. So if we let $\Delta_{j}^{\prime \prime}(t, x)$ be the discriminant of $q_{j}^{(2)}(t, x, \tau)$ as a polynomial in $\tau, \Delta_{j}^{\prime \prime} \in F_{1,1}$ near the origin which means an above factorization of $q_{3 j}$ is possible. Let us consider the case that $\widetilde{\Delta}_{3 j}(0,0)=0=\widetilde{\Delta}_{j}^{\prime}(0,0)$. We first note that it is always true in this case that when $(t, x)$ is close to $(0,0)$,

$$
\partial_{\tau} q_{3 j}(t, x, \tau)=3 \prod_{k=1}^{2}\left(\tau-\mu_{j k}^{\prime}(t, x)\right), \quad \mu_{j k}^{\prime} \in C_{2}^{\infty} \quad \text { and } \quad \mu_{j 1}^{\prime}-\mu_{j 2}^{\prime} \in F_{2,1}
$$

near $(0,0)$,

$$
q_{3 j}(t, x, \tau)=\left(\tau-\mu_{j k}^{\prime}(t, x)\right)^{2}\left(\tau-\mu_{j k}^{\prime}(t, x)+(-1)^{k} c_{j}(t, x)\right)+d_{j k}(t, x)
$$

where 


$$
c_{j}(t, x)=3 / 2\left(\mu_{j 2}^{\prime}(t, x)-\mu_{j 1}^{\prime}(t, x)\right), \quad d_{j k}(t, x)=q_{3 j}\left(t, x, \mu_{j k}^{\prime}(t, x)\right) .
$$

We also note that the well known relation between the discriminant of $q_{3 j}$ and $\partial_{\tau} q_{3 j}$ that

$$
1 / 27 \tilde{\Delta}_{3 j}=\prod_{k=1}^{2} \prod_{l=1}^{3}\left(\mu_{j k}^{\prime}-\mu_{3 j l}\right)=\prod_{k=1}^{2} q_{3 j}\left(t, x, \mu_{j k}^{\prime}\right)=\prod_{k=1}^{2} d_{j k}
$$

For this relation we refer B.L. van der Waerden: Algebra I. Clearly $\mu_{j k}^{\prime} \in C^{\infty}$ near $(0,0)$ when $\widetilde{\Delta}_{3 j} \equiv 0$ and $\widetilde{\Delta}_{j}^{\prime} \equiv 0$, and then $q_{3 j}(t, x, \tau)=\left(\tau-\mu_{j 1}^{\prime}(t, x)\right)^{3}$. When $\tilde{\Delta}_{3 j} \equiv 0$ and $\tilde{\Delta}_{j}^{\prime} \neq 0, \prod_{k=1}^{2} d_{j k}(t, x)=0$ for all $(t, x) \in\left(0, \varepsilon_{1}\right) \times\left(-\varepsilon_{1}, \varepsilon_{1}\right)$ with a small $\varepsilon_{1}$. Since both of two sets $\left\{(t, x) \in\left(0, \varepsilon_{1}\right) \times\left(-\varepsilon_{1}, \varepsilon_{1}\right) ; d_{j k}(t, x)=0\right\}, k=1,2$ are open in $\left(0, \varepsilon_{1}\right) \times\left(-\varepsilon_{1}, \varepsilon_{1}\right)$ if $\varepsilon_{1}$ is sufficiently small, for $\widetilde{\Delta}_{j}^{\prime} \in F_{1,1}^{*}$, one of them is empty. Thus by the above remark a desired factorization of $q_{3 j}$ is possible and by a change of numbering if neccessary, we have $d_{j 1}=0$ and $d_{j 2}(t, x)=-4 / 27 c_{j}(t, x)^{3}$ which means $d_{j 2} \in F_{2,1}^{*}$. When $\widetilde{\Delta}_{3 j} \neq 0$, by Lemma 1 one can find a positive integer $h$ and $\mu_{3 j k} \in C_{h}^{\infty}$ near $(0,0)$ such that $q_{3 j}(t, x, \tau)=\prod_{k=1}^{3}\left(\tau-\mu_{3 j k}(t, x)\right)$. Note that $\tilde{\Delta}_{3 j}(t, x)=\prod_{k>k^{\prime}}\left(\mu_{3 j k}(t, x)-\mu_{3 j k^{\prime}}(t, x)\right)^{2}=27 \prod_{k=1}^{2} d_{j k}(t, x)$, and that $\mu_{3 j k}\left(t^{2 h}, x\right)$ is $C^{\infty}$ in a neighbourhood of the origin in $\boldsymbol{R}^{2}$. Since $\widetilde{\Delta}_{3 j} \in F_{1,1}^{*}$ near $(0,0), \tilde{\Delta}_{3 j}(t, x)=t^{s} \tilde{\tilde{\Delta}}_{3 j}(t, x)$ in a neighbourhood of $(0,0)$ in $\boldsymbol{R}^{2}$ for some positive integer $s$ and $C^{\infty}$ function $\tilde{\tilde{\Delta_{3 j}}}$ with $\tilde{\tilde{\Delta}}_{3 j}(0,0) \neq 0$. So for all $k \neq k^{\prime}, \mu_{3 j k}\left(t^{2 h}, x\right)$ $-\mu_{3 j k^{\prime}}\left(t^{2 h}, x\right)$ has a finite order zero at $(0,0)$, and by Margrange's preperation theorem, in a neighbourhood of $(0,0)$ in $\boldsymbol{R}^{2}$

$$
\mu_{3 j k}\left(t^{2 h}, x\right)-\mu_{3 j k^{\prime}}\left(t^{2 h}, x\right)=\tilde{\mu}_{j k k^{\prime}}(t, x)\left(t^{s_{j k k^{\prime}}}+\sum_{l<s_{j k k^{\prime}}} c_{j k k^{\prime}}(x) t^{l}\right)
$$

for some positive integer $s_{j k k^{\prime}}$ and $C^{\infty}$ functions $\tilde{\mu}_{j k k^{\prime}}, c_{j k k^{\prime}}$ with $\tilde{\mu}_{j k k^{\prime}}(0,0) \neq 0$. Compairing orders of zeros at $(0,0)$ in the above equality, we see $h s=\sum_{k>k^{\prime}} s_{j k k^{\prime}}$, so we must have $c_{j k k^{\prime}} \equiv 0$ in a neighbourhood of the origin, since $\widetilde{\Delta}_{3 j}(t, x)=O\left(t^{s}\right)$ for all $x$ close to 0 . This means $\mu_{3 j k}-\mu_{3 j k^{\prime}} \in F_{2 h, 1}^{*}$ near $(0,0)$ for all $k \neq k^{\prime}$. Similar argument shows that $d_{j k} \in F_{2 h, 1}^{*}$ near $(0,0)$ for all $k$. Therefore a desired factorization of $q_{3 j}$ is possible. When the multiplicity of $\mu_{i j k}(t, x)$ is constant in $(t, x)$ near $(0,0)$ for some $(i, j, k), \mu_{i j k}(t, x)$ is $C^{\infty}$ in $(t, x)$ from the above argument.

In the latter case we perform following changes of coordinates with some $\delta>0$ succesively,

$$
\begin{aligned}
& t=t^{\prime}+\theta_{i_{0}}\left(x^{\prime}+x_{1}\right), \quad x=x^{\prime}+x_{1}, \quad\left|t^{\prime}\right| \leq 2 \delta, \quad\left|x^{\prime}\right| \leq 2 \delta \\
& t^{\prime}=\tilde{t} f(\tilde{x}), \quad x^{\prime}=\tilde{x}, \quad|\tilde{t}| \leq 2 \delta, \quad|\tilde{x}| \leq 2 \delta,
\end{aligned}
$$


where $f \in C_{0}^{\infty}(\boldsymbol{R})$ defined by

$$
f(s)=\exp \left(-\left(\delta^{2}-s^{2}\right)^{-1}\right) \text { when }|s|<\delta,=0 \text { when }|s| \geq \delta .
$$

From now on, $\delta$ denotes a fixed positive real number and $f(s)$ always denotes this function in this paper. We shall introduce some notation.

$B_{T}=\left\{a(t, x) \in C^{\infty}([0, T] \times R) ; \quad\right.$ Derivatives of $a$ of all orders are bounded\},

$$
\begin{aligned}
& B_{h, T}=\left\{a(t, x) \in C([0, T] \times R) ; a\left(t^{h}, x\right) \in B_{T}^{1 / h}\right\}, \\
& E_{h, T}=\left\{a(t, x) \in B_{h, T} ; a \equiv 0, \text { or } a(t, x)=(t f(x))^{e} \tilde{a}(t, x), e h \in N,\right. \\
& \left.\tilde{a} \in B_{h, T} \text { with inf }|\tilde{a}|>0\right\} .
\end{aligned}
$$

Then by denoting new variables $(\tilde{t}, \tilde{x})$ by $(t, x)$ anew and by a simple extention argument by the substitution into the variable $x$ of real valued $\varphi \in C^{\infty}(\boldsymbol{R})$ such that $|\varphi(s)|=3 \delta / 2$ when $|s| \geq 7 \delta / 4,3 \delta / 2 \geq|\varphi(s)| \geq 5 \delta / 4$ when $7 \delta / 4 \geq|s| \geq$ $5 \delta / 4, \varphi(s)=s$ when $|s|<5 \delta / 4$, in the latter case our proof of Theorem is reduced to the following Proposition.

Proposition 2.3. Let $\zeta \in B_{T_{0}}$ be real valued, $h$ an integer $>1$, and $p_{m}(t, x, \tau, \xi)$ a homogeneous polynomial in $(\tau, \xi)$ of degree $m \geq 1$ with $B_{T_{0}}$ coefficients such that

$$
p_{m}(t, x, \tau, \xi)=\prod_{i=1}^{3} \prod_{j=0}^{k_{i}} p_{i j}(t, x, \tau, \xi),(t, x, \tau, \xi) \in\left[0, T_{0}\right] \times \boldsymbol{R} \times \boldsymbol{R}^{2}, k_{i} \geq 0
$$

where $p_{i 0}=1$, and for $j \neq 1, p_{i j}$ is a homogeneous polynomial in $(\tau, \xi)$ of degree $i$ with $B_{T_{0}}$ coefficients,

for wihch we suppose that

$$
\begin{aligned}
& p_{i j}(t, x, \tau, \xi)=\prod_{k=1}^{i}\left(\tau-\mu_{i j k}(t, x) \xi\right),(t, x, \tau, \xi) \in\left[0, T_{0}\right] \times \boldsymbol{R} \times \boldsymbol{R}^{2} \text { with } \\
& \mu_{1 j 1} \in B_{h, T_{0}}, \mu_{i j k} \in B_{h, T_{0}} \text { for } i \neq 1, \text { satisfying that } \\
& \inf _{t, x}\left|\operatorname{Im} \mu_{i j k}(t, x)\right|>0 \text { for all }(i, j, k) \\
& \inf _{t, x}\left|\mu_{i j k}(t, x)-\mu_{i^{\prime} j^{\prime} k^{\prime}}(t, x)\right|>0 \text { if } i \neq i^{\prime}
\end{aligned}
$$

and that

$$
\mu_{i j k}-\mu_{i j k^{\prime}} \in E_{h, T_{0}} \text { for all }(i, j, k),\left(i, j, k^{\prime}\right) .
$$

We also suppose that for $j \neq 1$,

$$
\partial_{\tau} p_{3 j}(t, x, \tau, \xi)=3 \prod_{k=1}^{2}\left(\tau-\mu_{j k}^{\prime}(t, x) \xi\right),(t, x, \tau, \xi) \in\left[0, T_{0}\right] \times \boldsymbol{R} \times \boldsymbol{R}^{2}
$$


with

$$
\begin{aligned}
& \mu_{j k}^{\prime} \in B_{h, T_{0}}, k=1,2 \text { satisfying that } \\
& \underset{\omega}{\inf }\left|\operatorname{Im} \mu_{j k}^{\prime}(t, x)\right|>0 \text { for all } k, \mu_{j 1}^{\prime}-\mu_{j 2}^{\prime} \in E_{h, T_{0}}, \text { and that } \\
& p_{3 j}\left(t, x, \mu_{j k}^{\prime}(t, x), 1\right) \in E_{h, T_{0}} \text { for all } k .
\end{aligned}
$$

Then if $Q$ is a differential operator with the symbol $Q(t, x, \tau, \xi)=\tilde{Q}(t, x, \tau, f(x) \xi)$,

$$
\tilde{Q}(t, x, \tau, \xi)=p_{m}(t, x, \tau, \zeta(t, x) \tau+\xi)+f(x)^{1 / 2} p_{m-1}(t, x, \tau, \xi)+r(t, x, \tau, \xi),
$$

where $p_{m-1}$ and $r$ are respectively a homogeneous polynomial in $(\tau, \xi)$ of degree $m-1$ with $B_{T_{0}}$ coefficients and a polynomial in $(\tau, \xi)$ of degree $<m-1$ with $B_{T_{0}}$ coefficients, where we suppose $r=0$ if $m=1$, there exists $T_{1} \in\left(0, T_{0}\right)$ such that every $u \in C^{\infty}\left(\left[0, T_{0}\right] \times \boldsymbol{R}\right)$ which is flat on $t=0,=0$ when $|x| \geq \delta$, and satisfies $Q u=0$ vanishes when $t \leq T_{1}$.

Proposition 2.3 follows from a Carleman estimate: there exists $C>0$ such that for large $n>0$ and small $T>0$,

$$
C\|Q u\|^{(n)} \geq\|u\|^{(n)}, u \in \mathcal{S}_{T}, \quad \text { where }
$$

(2.11) $\mathcal{S}_{T}=\left\{u \in C^{\infty}((0, T) \times R) ; \sup (1+|x|)^{k} t^{-k}(T-t)^{-k}\left|\partial_{t}^{i} \partial_{x}^{j} u\right|<\infty\right.$ for all non negative integers $i, j, k\}$,

$$
\|u\|^{(n)}=\left\|t^{-n} u\right\|_{L^{2}((0, T) \times R)}, n \geq 1, u \in \mathcal{S}_{T} .
$$

To deduce (2.10) we shall prove that when under the assumption and notation of Proposition 2.3 with $m \leq 3, k_{m}=1, r=0$, there exists $C>0$ such that for large $n$ and small $T$,

$$
C\|Q u\|^{(n)} \geq\|u\|_{m}^{(n)} \quad \text { for } \quad u \in \mathcal{S}_{T},
$$

$$
C\left(\|Q u\|^{(n-1 /(20 h))}+n^{-1 / 2}\|Q u\|^{(n)}\right) \geq\left.\sum_{|\nu|+|\mu|=1}\left\|Q_{\nu \mu} u\right\|\right|^{(n)}, u \in \mathcal{S}_{T}
$$

where

$$
\begin{gathered}
\|u\|_{m}^{(n)}=\sum_{j+2 k \leq 2 m} n^{m / 2-j / 2-k}\left\|\Lambda_{j / 2} D_{t}^{k} u\right\|^{(n+m-j / 2-k)}, \\
\Lambda_{s}=\left(\langle f \xi\rangle^{s}\right)(x, D),
\end{gathered}
$$

that is,

$$
\begin{aligned}
& \Lambda_{s} u(t, x)=1 / 2 \pi \iint e^{i x \xi-i y \xi}\langle f(x) \xi\rangle^{s} u(t, y) d y d \xi \\
& Q_{\mu_{\nu}}=\left[\left(t\left\langle t^{1+1 /(10 h)} f \xi\right\rangle^{-1 / 2}\right)^{-\mu_{1}+\nu_{1}}\langle f \xi\rangle^{\left(\mu_{2}-\nu_{2}\right) / 2} f^{\nu_{2}} \partial_{t, x}^{y}\right. \\
& \left.\left\{p_{m}(t, x, 1, \zeta(t, x))^{-1}\left(\partial_{\tau, \xi}^{\mu} \tilde{Q}\right)(t, x, \tau, f(x) \xi)\right\}\right]\left(t, x, D_{t}, D_{x}\right) .
\end{aligned}
$$


Our main task is to prove (2.13.3) which we will devote, and when $m \neq 3$, (2.13.m) seems already known. Once (2.13) and (2.14) is established, one can easily pass to (2.10) using standard partial fraction decomposition.

\section{§3. Factorization of Some Third Degree Polynomial}

Definition 3.0. Let $n \in N, T>0$. We define

$$
\boldsymbol{R}_{T}^{n}:=\left\{x=\left(x_{1}, \cdots, x_{n}\right) \in \boldsymbol{R}^{n} ; x_{1} \in(0, T)\right\} .
$$

Let $p_{3}(t, x, \tau, \xi)$ and $p_{2}(t, x, \tau, \xi),(t, x, \tau, \xi) \in \boldsymbol{R}_{T}^{4}$, be elements in $C_{T}^{\infty}\left(\boldsymbol{R}^{4}\right)$ being homogeneous polynomials in $(\tau, \xi)$ respectively of degrees 3 and 2 with coefficients in $B_{T}$ in $(t, x)$. Let $h \in N$. We suppose that

(3.1) $\partial_{\tau} p_{3}(t, x, \tau, \xi)=3 \prod_{l=1}^{2}\left(\tau-\mu_{l}(t, x) \xi\right), \mu_{l} \in B_{h, T} \quad$ with inf $\left|\operatorname{Im} \mu_{l}\right|>0$, $c:=3 / 2\left(\mu_{2}-\mu_{1}\right) \in E_{h, T}, d_{l}:=p_{3}\left(t, x, \mu_{l}(t, x), 1\right) \in E_{h, T}$.

Then we have

$$
p_{3}(t, x, \tau, \xi)=\left(\tau-\mu_{l}(t, x) \xi\right)^{2}\left(\tau-\mu_{l}(t, x) \xi+(-1)^{l} c(t, x) \xi\right)+d_{l}(t, x) \xi^{3} .
$$

Let $\zeta(t, x) \in B_{T}$ be real valued and put

$$
L_{l}=\tau-\mu_{l}(t, x)(\zeta(t, x) \tau+\xi), l=1,2 .
$$

Then we can express uniquely

$$
\begin{gathered}
p_{2}=\sum_{i=0}^{2} a_{i l} L_{l}^{i} \xi^{2-i}, a_{i l} \in B_{h, T}, \\
\zeta \tau+\xi=a_{l} L_{l}+b_{l} \xi, a_{l}, b_{l} \in B_{h, T} \quad \text { with } \inf _{t, x} \quad\left|b_{l}\right|>0,
\end{gathered}
$$

since $\zeta$ is real and $\inf _{t, x}\left|\operatorname{Im} \mu_{l}\right|>0$. We set

$$
\begin{gathered}
q=p_{3}(t, x, \tau, \zeta(t, x) \tau+\xi)+f(x)^{1 / 2} p_{2}(t, x, \tau, \xi), \\
g_{l}=d_{l} b_{l}^{3} \xi^{3}+a_{0 l} f^{1 / 2} \xi^{2}, \\
G_{l}=\left\langle g_{l}\right\rangle, H_{l}=\left\langle\left(c b_{l} \xi\right)^{3}\right\rangle,
\end{gathered}
$$

where $f$ is the function defined by (2.2) and $\langle z\rangle=\left(1+|z|^{2}\right)^{1 / 2}$. Then a direct computation shows

$$
\begin{aligned}
& q=L_{l}^{2}\left(L_{l}+(-1)^{l} c b_{l} \xi\right)+g_{l}+A_{0 l} L_{l}^{3}+d_{l} \sum_{i=1}^{3} A_{i l} L_{l}^{i} \xi^{3-i} \\
& \quad+f^{1 / 2} \sum_{i=1}^{2} a_{i l} L_{l}^{i} \xi^{2-i} \\
& A_{i l}=3 a_{l}^{i} b_{l}^{3-i} \text { for } i \neq 0, A_{0 l}=(-1)^{l} c a_{l}+d_{l} a_{l}^{3}
\end{aligned}
$$


Definition 3.1. Let $T \in(0,1)$ and $h \in N$. We say $a(t, x, \xi) \in C^{\infty}\left(\boldsymbol{R}_{T}^{3}\right)$ belongs to $\Sigma_{\rho}^{d}=\Sigma_{\rho}^{d}(T, h)$ for $\rho \in[0,1 / 2]$ and $d=\left(d_{1}, d_{2}, d_{3}\right)$ with $d_{1} \leq 0$, if $a$ satisfies

$$
\left|\partial^{\alpha} a(t, x, \xi)\right| \leq C_{\alpha} \psi_{1}^{d_{1}-\alpha_{1}} \cdot \psi_{2^{2}}^{d_{2}-\alpha_{2} \cdot \psi_{3, \rho}^{d_{3}-\alpha_{3}}} \text { for all } \alpha
$$

where $\partial^{\alpha}=\partial_{t}^{\alpha} \partial_{x^{2}}^{\alpha} \partial_{\xi}^{\alpha}$,

$$
\begin{aligned}
& \psi_{1}=t\left\langle t^{e}{ } f^{1 /(10 h)} \xi\right\rangle^{-1 / 2}, \psi_{2}=\left\langle t^{e}{ }_{h} \xi\right\rangle^{-1 / 2}, \\
& \psi_{3, \rho}=\left\langle t^{e}{ }^{e} \xi\right\rangle^{-\rho}\langle\xi\rangle, e_{h}=1+1 /(10 h) .
\end{aligned}
$$

Note that

$$
a b \in \Sigma_{\rho}^{d+e} \text { if } a \in \Sigma_{\rho}^{d}, b \in \Sigma_{\rho}^{e} .
$$

Let $\Delta(t, x)$ and $\Delta_{1}(t, x)$ be respectively the discriminants of $p_{3}(t, x, \tau, 1)$ and $\partial_{\tau} p_{3}(t, x, \tau, 1)$ as polynomials in $\tau$. Then similarly as (2.1),

$$
\Delta=27 \prod_{l=1}^{2} d_{l}, \quad \Delta_{1}=4 / 9 c^{2} .
$$

Since $c, d_{l} \in E_{h, T}$, the possible cases are the followings.

$$
\text { (1) } \Delta \neq 0 \text {. }
$$

(2) $\Delta \equiv 0, \Delta_{1} \neq 0$.

(3) $\Delta \equiv 0, \Delta_{1} \equiv 0$.

So from the definition of $E_{h, T}$,

(3.14) the only one of $d_{1}$ and $d_{2}$ is identically zero in case (2), and we have

(3.15) $d_{l} \equiv 0$ for any $l$ and $\mu_{1} \equiv \mu_{2}$ in case (3)

so that

(3.16) we delete sub-1 from all above notations in case (3), since in fact they do not depend on $l$ in this case, namely we denote $\mu_{l}$ by $\mu$ and $L_{l}$ by $L$ for example.

Since $c, d_{l} \in E_{h, T}$ it follows from (3.9)

(3.17) $2 \geq\left|1+A_{0 l}\right| \geq 1 / 2$ when $t \leq T^{\prime}$ for some $T^{\prime} \in(0, T)$.

The aim of this section is to prove the following lemma.

Lemma 3.2. Let $T^{\prime}$ be as in (3.17). For each cases in (3.13) the following facts holds for some $T_{0} \in\left(0, T^{\prime}\right)$.

Case (1). There exists a finite partitions of unity of $\boldsymbol{R}_{T_{0}}^{3}, \sum_{i \in I} \varphi_{i}+\sum_{l=1}^{2} \varphi_{0 l}=1$ such that

$$
\varphi_{i}, \varphi_{0 b} \in \Sigma_{1 / 2}^{0}\left(T_{0}, h\right)
$$




$$
\left|g_{l}\right| \leq C t^{-\kappa}\langle\xi\rangle^{3 / 2} \text { on } \operatorname{supp} \varphi_{0 l}
$$

where

$$
\kappa=3 / 2-1 /(3 h) ;
$$

(3.20) $C\langle\xi\rangle \geq t^{-2 \kappa / 3}$ on supp $\varphi_{i}$ and on supp $d \varphi_{0 l}$; for every $i \in I$ there exists $l=l(i) \in\{1,2\}$ such that

$$
\begin{aligned}
& q=\left(1+A_{0 l}\right) \prod_{j=1}^{3}\left(L_{l}-\sigma_{i j}\right) \text { if }(t, x, \xi) \in \operatorname{supp} \varphi_{i} \\
& C\left|g_{l}\right| \geq t^{-\kappa}\langle\xi\rangle^{3 / 2} \quad \text { on supp } \varphi_{i} \text { with }
\end{aligned}
$$

(3.22) $\sigma_{i j} \in t^{1 /(6 h)} \Sigma_{1 / 2}^{(0,-1,1)}$,

$$
\partial_{t} \sigma_{i j} \in t^{1 /(6 h)-1} \Sigma_{1 / 2}^{(0,-1,1)}, \partial_{x} \sigma_{i j} \in t^{1 /(6 h)} \Sigma_{1 / 2}^{(0,-1,1)}, \partial_{\xi} \sigma_{i j} \in t^{1 /(6 h)} \Sigma_{1 / 2}^{0}
$$

where by definition for a topological space $X$

$$
a E=\{a e ; e \in E\} \quad \text { for } a \in C(X) \text { and } E \subset C(X) .
$$

Case (2). There exists a finite partitions of unity of $\boldsymbol{R}_{T_{0}}^{3}, \Sigma_{i \in I} \varphi_{i}+\Sigma_{l=1}^{2} \varphi_{0 l}=1$ such that (3.18) (3.20) hold, and such that $I=I_{1} \cup I_{2}$ with $I_{1} \cap I_{2}=\phi$ so that for every $i \in I_{1}$ (3.21) holds for some $l=l(i)$ and

$$
\sigma_{i j} \in \Sigma_{1 / 2}^{2 / 3(0,-1,1)} \quad \text { satisfying (3.22) }
$$

and for every $i \in I_{2}$ there exists $l=l(i) \in\{1,2\}$ and $\sigma_{i j}$ satisfying (3.23) such that

$$
q=\left(1+A_{01}\right)\left(L_{l}-c_{l} \xi-\sigma_{i 1}\right) \prod_{j=2}^{3}\left(L_{l}-\sigma_{i j}\right) \quad \text { if } \quad(t, x, \xi) \in \operatorname{supp} \varphi_{i}
$$

where

$$
c_{l}=(-1)^{l+1}\left(1+A_{0 l}\right)^{-1} b_{l} c .
$$

Moreover the following facts hold.

$$
\begin{aligned}
& \left|g_{l(i)}\right| \leq C\langle\xi\rangle^{2} \text { on supp } \varphi_{i}, i \in I, \\
& |c| \leq C\langle\xi\rangle^{-1 / 3} \text { on } \operatorname{supp} \varphi_{i}, i \in I_{1}, \\
& c \xi \cdot \sigma_{i j} \in \Sigma_{1 / 2}^{3 / 2(0,-1,1)} \text { for } j=1,2,3, i \in I_{2} .
\end{aligned}
$$

Case (3). There exists a finite partitions of unity of $\boldsymbol{R}_{T_{0}}^{3}, \sum_{i \in I} \varphi_{i}+\varphi_{0}=1$ such that (3.18) (3.19) replaced $\varphi_{0 l}$ by $\varphi_{0}$ hold and (3.21) hold for some

$$
\sigma_{i j} \in \Sigma_{0}^{2 / 3(0,0,1)} \quad \text { satifying }
$$

(3.22) $\sigma_{i j} \in t^{1 /(6 h)} \Sigma_{0}^{(0,0,1)}$, 


$$
\partial_{t} \sigma_{i j} \in t^{1 /(6 h)-1} \Sigma_{0}^{(0,0,1)}, \partial_{x} \sigma_{i j} \in t^{1 /(6 h)} \Sigma_{0}^{(0,0,1)}, \partial_{\xi} \sigma_{i j} \in t^{1 /(6 h)} \Sigma_{0}^{0}
$$

Furthermore there exists $\varphi_{i}^{*} \in \Sigma_{0}^{0}$ such that

$$
\begin{aligned}
& \varphi_{i}^{*}=1 \quad \text { on } \operatorname{supp} \varphi_{i} \\
& C\left|\sigma_{i j}-\sigma_{i k}\right| \geq \max _{s}\left|\sigma_{i s}\right|+t^{-\kappa / 3}\langle\xi\rangle^{1 / 2} \quad \text { on } \operatorname{supp} \varphi_{i}^{*} \text { if } j \neq k
\end{aligned}
$$

Proof. We may assume $T^{\prime}<1$. For $S \in\left(0, T^{\prime}\right)$ and $\varepsilon \in(0,1)$ we set

$$
\begin{aligned}
& \Gamma_{l}(S)=\left\{(t, x, \xi) \in \boldsymbol{R}_{S}^{3} ; t^{\kappa} G_{l}>\langle\xi\rangle^{3 / 2}\right\}, \\
& \Gamma^{l, i}(S, \varepsilon)=\Gamma_{l}(S) \cap \mathscr{D}_{l}^{(i)}(\varepsilon) \cap \mathcal{D}^{l},
\end{aligned}
$$

for $l=1,2, i=1,2,3$ where

$$
\begin{aligned}
& \mathscr{D}_{l}^{(1)}=\left\{G_{l}<10 \varepsilon H_{l}\right\}, \mathscr{D}_{l}^{(2)}=\left\{H_{l}<10 \varepsilon G_{l}\right\}, \\
& \mathscr{D}_{l}^{(3)}=\left\{\frac{\varepsilon}{10} H_{l}<G_{l}<\frac{10}{\varepsilon} H_{l}\right\}, \mathscr{D}^{l}=\left\{G_{l^{\prime}}>10^{-1} G_{l}\right\} \text { with } l \neq l^{\prime} .
\end{aligned}
$$

Then,

$$
\begin{aligned}
& 2^{-1} \leq\left|c b_{l} \xi\right| H_{l}^{-1 / 2} \leq 1 \text { on } \Gamma^{l, 1}(S, \varepsilon) \cup \Gamma^{l, 3}(S, \varepsilon) \text { if } \varepsilon S^{-\kappa}>20, \\
& 2^{-1} \leq\left|g_{l}\right| G_{l}^{-1} \leq 1 \text { on } \Gamma_{l}(S) \text { if } S^{-k}>2 \\
& \Gamma^{l, 1}(S, \varepsilon)=\Gamma^{l, 3}(S, \varepsilon)=\emptyset \text { if } \varepsilon S^{-\kappa}>10 \text { and } c \equiv 0 ;
\end{aligned}
$$

and there exist constant $C>0$ and $S_{0} \in\left(0, T^{\prime}\right)$ such that

$$
\begin{aligned}
& \max \left(2, C^{-1} t^{-2 \kappa / 3}\right)<\langle\xi\rangle \text { on } \Gamma_{l}(S) \text { for } S \in\left(0, S_{0}\right), \\
& S^{\max (1 / h, 2 \kappa / 3)} C G_{l}^{2 / 3}>\left|d_{l}\right|\langle\xi\rangle^{2} \text { on } \Gamma_{l}(S) \text { for } S \in\left(0, S_{0}\right) .
\end{aligned}
$$

Indeed, $H_{l}>\varepsilon S^{-\kappa} / 10$ on $\Gamma^{l, i}(S, \varepsilon)$ for $i=1,3$ and $G_{l}>S^{-\kappa}$ on $\Gamma_{l}(S)$ which means (3.31), since $\langle z\rangle \geq 2, z \in C$ implies $|z| \geq 1$. To see (3.32) we set for $S \in(0, T]$,

$$
|a|_{0, S}=\max _{[0, S] \times \boldsymbol{R}}|a(t, x)|, a \in B_{h, T} .
$$

It suffices to prove separately for each of (3.32) the existence of $C$ and $S_{0}$. Since $d_{l} \in E_{h, T}, d_{l}=t^{1 / h} \tilde{d_{l}}$ for some $\tilde{d_{l}} \in B_{h, T}$ so that $\left|g_{l}\right| \leq S^{1 / h}\left|\tilde{d}_{l}\right|_{0, s}|b|_{0, s}{ }^{3}\langle\xi\rangle^{3}+$ $\left|a_{0 l}\right|_{0, s}\langle\xi\rangle^{2}$ by using $0 \leq f \leq 1$. Thus there exists $C_{1}>0$ such that $\left.C_{1} t^{k}\langle\xi\rangle^{3}\right\rangle$ $\langle\xi\rangle^{3 / 2}$ on $\Gamma_{l}(S)$ for any $S$ which shows the first one of (3.32) and there exists $C_{2}$ such that $\left|g_{l}\right| \leq C_{2} S^{\min (1 / h, 2 \kappa / 3)}\langle\xi\rangle^{3}$ on $\Gamma_{l}(S)$ for any $S$. On the other hand $\left|g_{l}\right| \geq\left|b_{b}\right|^{3}\left|d_{l}\right||\xi|^{3}-\left|a_{0 l}\right||\xi|^{2}$ so that

$$
\begin{aligned}
& \left.\inf _{[0, T] \times \boldsymbol{R}}\left|b_{l}\right|\right)^{3}\left|d_{l}\right||\xi|^{3}-\left|a_{0 l}\right|_{0, s}\langle\xi\rangle t^{2 \kappa / 3} G_{l}^{2 / 3} \\
& \leq C_{2}^{1 / 3} S^{\min (1 / h, 2 \kappa / 3)} G_{l}^{2 / 3}\langle\xi\rangle \quad \text { on } \Gamma_{l}(S)
\end{aligned}
$$


in view of the definition of $\Gamma_{l}(S)$. This shows the second one of (3.32) in view of $\inf _{\left[0, T^{\prime}\right] \times \boldsymbol{R}}\left|b_{l}\right|>0$ and the first one of that.

Putting $L_{l}=H_{l}^{1 / 3} \sigma$ or $L_{l}=G_{l}^{1 / 3} \sigma$ in (3.9) we get

$$
\begin{aligned}
& \tilde{q}\left(H_{l}^{1 / 3} \sigma, \cdot\right)=\left(1+A_{0 l}\right) H_{l} r_{1}\left(\sigma, z^{l 1}, w^{l 1}\right), \\
& \tilde{q}\left(G_{l}^{1 / 3} \sigma, \cdot\right)=\left(1+A_{0 l}\right) G_{l} r_{2}\left(\sigma, z^{l 2}, w^{l 2}\right), \\
& \tilde{q}\left(H_{l}^{1 / 3} \sigma, \cdot\right)=H_{l} r_{3}\left(\sigma, z^{l 3}, w^{l 3}\right),
\end{aligned}
$$

where $\tilde{q}(\sigma, t, x, \xi)$ is the polynomial in defined by putting $L_{1}=\sigma$ in (3.9),

$$
\begin{aligned}
& r_{1}(\sigma, z, w)=\sigma^{3}+\left(w_{1}+w_{2} z\right) \sigma^{2}+w_{3} z^{2} \sigma+w_{4} z^{3}, \quad(z, w) \in \boldsymbol{C} \times \boldsymbol{C}^{4}, \\
& r_{2}(\sigma, z, w)=\sigma^{3}+w_{1} \sigma^{2}+w_{2} \sigma+z, \quad(z, w) \in \boldsymbol{C} \times \boldsymbol{C}^{2}, \\
& r_{3}(\sigma, z, w)=\left(1+w_{1}\right) \sigma^{3}+\left(w_{2}-z_{1}\right) \sigma^{2}+w_{3} \sigma+z_{2}, \quad(z, w) \in \boldsymbol{C}^{2} \times \boldsymbol{C}^{3} ; \\
& z^{l 1}=G_{l}^{1 / 3} H_{l}^{1 / 3}, w^{l 1}=\left(1+A_{0 l}\right)^{-1}\left(-\tilde{c}_{l} \xi H_{l}^{-1 / 3}, G_{l}^{-1 / 3} v_{2}, G_{l}^{-2 / 3} v_{l}, g_{l} G_{l}^{-1}\right), \\
& z^{l 2}=\left(1+A_{0 l}\right)^{-1} g_{l} G_{l}^{-1}, w^{l 2}=\left(1+A_{0 l}\right)^{-1}\left(-\tilde{c}_{l} \xi G_{l}^{-1 / 3}+G_{l}^{-1 / 3} v_{2}, G_{l}^{-2 / 3} v_{1}\right), \\
& z^{l 3}=\left(\tilde{c}_{l} \xi H_{l}^{-1 / 3}, g_{l} H_{l}^{-1}\right), w^{l 3}=\left(A_{0 l}, H_{l}^{-1 / 3} v_{2}, H_{l}^{-2 / 3} v_{1}\right), \\
& v_{i}=d_{l} A_{i l} \xi^{3-i}+f^{1 / 2} a_{i l} \xi^{2-i}, \tilde{c}_{l}=(-1)^{l+1} c b_{l} .
\end{aligned}
$$

\section{Lemma 3.3. Set for $\eta \in(0,1)$,}

$$
\begin{aligned}
X_{1, \eta}= & {[0, \eta] \times\left\{w \in \boldsymbol{C}^{4} ; \frac{1}{10} \leq\left|w_{j}\right| \leq 10 \text { for } j=1,4, \max _{j=2,3}\left|w_{j}\right| \leq 1\right\}, } \\
X_{2, \eta}= & \left\{z \in \boldsymbol{C} ; \frac{1}{10} \leq|z| \leq 10\right\} \times\left\{w \in \boldsymbol{C}^{2} ;\left|w_{1}\right| \leq \eta,\left|w_{2}\right| \leq \eta\right\}, \\
X_{3, \eta}= & \left\{(z, w) \in \boldsymbol{C}^{2} \times \boldsymbol{C}^{3} ; \frac{1}{10} \leq|z| \leq 10, \frac{\eta}{100} \leq\left|z_{2}\right| \leq \frac{100}{\eta}, \max _{j}\left|w_{j}\right| \leq \eta,\right. \\
& \left.\left|D_{3}(z, w)\right| \geq \frac{\eta^{2}}{500}\right\},
\end{aligned}
$$

where $D_{3}(z, w)$ denotes the discriminant of $r_{3}$ as a polynomial in $\sigma$. Then there exists $\eta_{0} \in(0,1)$ such that one can find for any $\eta \leq \eta_{0}$ finite open coverings $U_{i j}$ of $X_{i, \eta}$ as a subset of $\boldsymbol{C} \times \boldsymbol{C}^{4}, \boldsymbol{C} \times \boldsymbol{C}^{2}, \boldsymbol{C}^{2} \times \boldsymbol{C}^{3}$ respectively for $i$, holomorphic functions $\lambda_{i j k}$ on $U_{i j}, k=1,2,3$ for $i \neq 1, C^{\infty}$ functions $\lambda_{1 j k}(t, w)$ on $U_{1 j} \cap(0,+\infty) \times C^{4}$ satisfying

(3.37) $U_{1 j}=B\left(\left(0, w^{(j)}\right), \nu\right), w_{1}^{(j)} \neq 0, \nu<1$ where $B(*, \nu)$ denotes the Euclidean open ball with the center $*$ and the radius $\nu$,

$$
\begin{aligned}
& r_{1}(\sigma, t, w)=\prod_{k=1}^{3}\left(\sigma-\lambda_{1 j k}(t, w)\right),(t, w) \in U_{1 j} \cap(0,+\infty) \times \boldsymbol{C}^{4}, \\
& \left|\partial_{t}^{s} \partial_{w}^{\alpha}\left(\lambda_{1 j 1}(t, w)+w_{1}\right)\right| \leq C_{s \alpha} t^{1-s}, \\
& \left|\partial_{t}^{s} \partial_{w}^{\alpha} \lambda_{1 j k}(t, w)\right| \leq C_{s \alpha} t^{3 / 2-s} \text { for } k \neq 1,
\end{aligned}
$$




$$
\begin{aligned}
& r_{2}(\sigma, \cdot)=\prod_{k=1}^{3}\left(\sigma-\lambda_{2 j k}\right) \text { on } U_{2 j}, \\
& r_{3}(\sigma, \cdot)=\left(1+w_{1}\right) \prod_{k=1}^{3}\left(\sigma-\lambda_{3 j k}\right) \text { on } U_{3 j}, \\
& \lambda_{i j k} \neq \lambda_{i j k^{\prime}} \text { everywhere on } U_{i j} \text { if } i \neq 1 \text { and } k \neq k^{\prime}, \\
& \left|\partial_{z, w}^{\alpha} \lambda_{i j k}\right| \leq C_{\alpha} \text { on } U_{i j} \text { if } i \neq 1, \\
& C\left|\lambda_{2 j k}-\lambda_{2 j k^{\prime}}\right| \geq \max _{s}\left|\lambda_{2 j s}\right|+1 \text { on } U_{2 j} \text { if } k \neq k^{\prime},
\end{aligned}
$$

(3.40) $\sum_{j} \varphi_{i j}=1$ on a neighbourhood of $X_{i, \eta}$ and $\varphi_{i j}^{*}=1$ on $\operatorname{supp} \varphi_{i j}$,

where the constants $C_{s a}, C$ can be chosen independently of $\eta$, but it is not important in our argument.

Proof of Lemma 3.3. We first consider the case $i=1$. From the implicit function theorem, for any $w^{0} \in \mathbb{C}^{4}$ with $w_{j}^{0} \neq 0$ for $j=1,4$ one can write near $\left(0, w^{0}\right)$ $r_{1}=(\sigma+\lambda)\left(\sigma^{2}+\lambda_{1} \sigma+\lambda_{2}\right)$ where $\lambda, \lambda_{i}$ are holomorphic and $\lambda(0, w)=-w_{1}$, because $r_{1}(\sigma, 0, w)=\sigma^{3}+w_{1} \sigma^{2}$. Since $w_{4} z^{3}=r_{1}(0, z, w)=\lambda \lambda_{2}$ and $w_{3} z^{2}=\partial_{\sigma} r_{1}(0, z, w)=\lambda_{2}$ $+\lambda \lambda_{1}$, and since $w_{4}^{0} \neq 0$ and $\lambda\left(0, w^{0}\right) \neq 0$, near $\left(0, w^{0}\right), \lambda_{1}=O\left(z^{2}\right)$ as $z \rightarrow 0$ and one can write $\lambda_{1}^{2}-4 \lambda_{2}=z^{3} \lambda_{3}$ where $\lambda_{3}$ is holomorphic with $\lambda_{3}\left(0, w^{0}\right) \neq 0$. So the factorization as in (3.37) is possible near $\left(0, w^{0}\right)$, and for $i=1$ Lemma 3.3 follows now by a standard argument by using the compactness of $\left\{w \in C^{4} ; \frac{1}{10} \leq\left|w_{j}\right|\right.$ $\leq 10$ for $\left.j=1,4, \max _{j=2,3}\left|w_{j}\right| \leq 1\right\}$. When $i=2$, for any $z^{0} \in C \backslash\{0\}$ one can factorize $r_{2}(\sigma, \cdot)$ near $\left(z^{0}, 0\right)$ so that (3.38), (3.39) hold, since $r_{2}(\sigma, z, 0)=\sigma^{3}+z$, and Lemma 3.3 for $i=2$ follows from the compactness of $\left\{z \in C ; \frac{1}{10} \leq|z| \leq 10\right\}$. The case that $i=3$ also follows similarly, since $D_{3} \neq 0$ on $X_{3, \eta}$ and $X_{3, \eta}$ is compact. The proof is complete.

Lemma 3.4. There exists $\varepsilon_{0}, \varepsilon_{1} \in(0,1)$ with $\varepsilon_{1}<\varepsilon_{0}$ and $N_{0} \in[1, \infty)$ such that $\varepsilon_{0}$ satisfies the condition for the $\eta_{0}$ in the statement of Lemma 3.3, that $\varepsilon_{0}^{1-N_{0} k}>20$, and that $\varepsilon_{0}^{N} 0<S_{0}$ where $S_{0}$ is the constant in (3.32), and such that for the sets

$$
\tilde{\Gamma}^{l, i}(\varepsilon)=\left\{\left(z^{l i}(t, x, \xi), w^{l i}(t, x, \xi)\right) ;(t, x, \xi) \in \Gamma^{l, i}\left(\varepsilon_{0}^{N}, \varepsilon\right)\right\}, \varepsilon \in\left(0, \varepsilon_{0}\right),
$$

we have for any $\varepsilon \in\left(0, \varepsilon_{1}\right)$ that

$$
\begin{aligned}
& \tilde{\Gamma}^{l, i}(\varepsilon) \subset X_{i, \varepsilon_{0}} \text { for } i=1,2 ; \\
& \tilde{\Gamma}^{l, 3}(\varepsilon) \subset X_{3, \varepsilon}
\end{aligned}
$$

Proof of Lemma 3.4. From (3.32) and the definition of $\Gamma_{l}(S)$ there exists $C_{1}^{-}$such that 


$$
\left|v_{i}\right| G_{l}^{-(3-i) / 3} \leq C_{1} S^{(3-i) / 6 \min (1 / h, 2 \kappa / 3)} \text { on } \Gamma_{l}(S) \text { for } S \in\left(0, S_{0}\right), i=1,2
$$

where $S_{0}$ is the constant in (3.32). Using the fact that $c, d_{l} \in E_{h, T}$ we see as in the proof of (3.32) that there exists $C_{2}$ such that

$$
\left|A_{01}\right|_{0, s} \leq C_{2} S^{1 / h}, S \in(0, T] .
$$

Using (3.17), (3.31), (3.42), (3.44) one can find $S_{1} \in\left(0, S_{0}\right)$ and $C_{3}$ such that for any $\varepsilon$ with $\varepsilon S^{-\kappa}>20$,

$$
\begin{aligned}
& 0<z^{l 1}<C_{3} \varepsilon^{1 / 3}, 1 / 4 \leq\left|w_{i}^{l 1}\right| \leq 2 \text { for } i=1,4, \max _{i=2,3}\left|w_{i}^{l 1}\right| \\
& \quad \leq C_{3} S^{1 / 6 \min (1 / h, 2 \kappa / 3)} \text {, on } \Gamma^{l, 1}(S, \varepsilon) ; \\
& 1 / 4 \leq\left|z^{l 2}\right| \leq 2,\left|w_{1}^{l 2}\right| \leq C_{3}\left(\varepsilon^{1 / 3}+S^{1 / 6 \min (1 /, 3 k / 3)}\right), \\
& \left|w_{2}^{l 2}\right| \leq C_{3} S^{1 / 3 \min (1 / h, 2 \kappa / 3)}, \text { on } \Gamma^{l, 2}(S, \varepsilon) ; \\
& 1 / 2 \leq\left|z_{1}^{l 3}\right| \leq 1, \varepsilon / 20<\left|z_{2}^{l 3}\right|<10 / \varepsilon,\left|w_{1}^{l 3}\right| \leq C_{3} S^{1 / h}, \\
& \left|w_{1}^{l 3}\right| \leq C_{3}\left(\varepsilon^{-1} S^{1 / 2 \min (1 / h, 2 \kappa / 3)}\right)^{(i-1) / 3} \text { for } i=2,3 \text {, on } \Gamma^{l, 3}(S, \varepsilon) .
\end{aligned}
$$

We take $N_{0}=\left(6 \operatorname{deg} D_{3}+20\right) / \min (1 / h, 2 \kappa / 3)$ so that $\varepsilon^{-1} S^{\min (1 / h, 2 \kappa / 3) / 2}=\varepsilon^{3\left(\operatorname{deg} D_{3}+3\right)}$ if $S=\varepsilon^{N_{0}}$, where $D_{3}$ is as in Lemma 3.3 and deg $D_{3}$ is the degree of a polynomial $D_{3}$. One can choose $\varepsilon_{0} \in(0,1)$ such that $\varepsilon_{0}^{N} 0\left\langle S_{1} \cdot \varepsilon_{0}^{1-N_{0} \kappa}\right\rangle 20$, and satisfies the condition for $\eta_{0}$ in the statement of Lemma 3.3, since $N_{0}>1$ and $\kappa>1$. Then it follows from (3.45) that for any $\varepsilon \in\left(0, \varepsilon_{0}\right)$,

$$
\begin{aligned}
& 0<z^{l 1} \leq C_{3} \varepsilon^{1 / 3}, 1 / 4 \leq\left|w_{i}^{l 1}\right| \leq 2 \\
& \quad \text { for } i=1,4, \max _{i=2,3}\left|w_{i}^{l 1}\right| \leq C_{3} \varepsilon, \text { on } \Gamma^{l, 1}\left(\varepsilon^{N_{0}}, \varepsilon\right) ; \\
& 1 / 4 \leq\left|Z^{l 2}\right| \leq 2,\left|w^{l 2}\right| \leq 2 C_{3} \varepsilon^{1 / 3}, \text { on } \Gamma^{l, 2}\left(\varepsilon^{N_{0}}, \varepsilon\right) ; \\
& 1 / 2 \leq\left|z_{1}^{l 3}\right| \leq 1, \varepsilon / 20 \leq\left|z_{2}^{l 3}\right| \leq 10 / \varepsilon,\left|w^{l 3}\right| \leq C_{3} \varepsilon^{3\left(\operatorname{deg} D_{3}+3\right)}, \text { on } \Gamma^{l, 3}\left(\varepsilon^{N_{0}}, \varepsilon\right) ;
\end{aligned}
$$

where $|w|=\max _{1 \leq j \leq n}\left|w_{j}\right|$ if $\quad w=\left(w_{j}\right) \in \boldsymbol{C}^{n}$.

So the first one of (3.42) is now clear, and we can choose $\varepsilon^{(1)} \in\left(0, \varepsilon_{0}\right)$ such that this holds for any $\varepsilon \in\left(0, \varepsilon^{(1)}\right)$. We have to estimate $\left|D_{3}(z, w)\right|$ from below on $\Gamma^{l, 3}\left(\varepsilon^{N_{0}}, \varepsilon\right)$ to see the second one of (3.42).

By Taylor expansion at $w=0$ of $D_{3}$ and (3.46) one can find $C_{4}$ such that for any $\varepsilon \in\left(0, \varepsilon_{0}\right)$

$$
\left|D_{3}\left(z^{l 3}, w^{l 3}\right)\right| \geq\left|D_{3}\left(z^{l 3}, 0\right)\right|-C_{4}\left(1+\varepsilon^{-1}\right)^{\operatorname{deg} D_{3}-1} \varepsilon^{\operatorname{deg} D_{3}+3} \text { on } \Gamma^{l, 3}\left(\varepsilon^{N_{0}}, \varepsilon\right) .
$$

We see as in (2 1),

$$
D_{3}(z, 0)=27 r_{3}(0, z, 0) r_{3}\left(2 z_{1} / 3, z, 0\right)=\left(27 z_{2}-4 z_{1}^{3}\right) z_{2}
$$


so that

$$
\left|D_{3}\left(z^{l 3}, 0\right)\right|=27\left|g_{l}\right| H_{l}^{-1}\left|g_{l}-\frac{4}{27}\left(\tilde{c}_{l} \xi\right)^{3}\right| H_{l}^{-1}
$$

To compute $g_{b}-\frac{4}{27}\left(\tilde{c}_{b} \xi\right)^{3}$ we first note that from the uniquness of the expression in (3.4) and (3.5) and $L_{1}-L_{2}=\frac{2 c}{3}(\zeta \tau+\xi)$,

$$
a_{01}-a_{02}, b_{1}-b_{2} \in c B_{h, T},
$$

for indeed, from (3.4) for $l=1$, (3.5) for $l=2$, and the above equality we have $p_{2}=\sum_{j=0}^{2}\left(a_{j 1}+c \tilde{a}_{j}\right) L_{2}^{j} \xi^{2-j}$ for some $\tilde{a}_{j} \in B_{h, T}$ and the uniqueness implies $a_{j 1}+$ $c \tilde{a}_{j}=a_{j 2}$, and the latter follows similarly. Next we verify that

$$
d_{2}=p_{3}\left(t, x, \mu_{2}, 1\right)=p_{3}\left(t, x, \frac{2 c}{3}+\mu_{1}, 1\right)=d_{1}-\frac{4}{27} c^{3} .
$$

From this equality and (3.49) we obtain by noting $\inf _{t, x}\left|b_{l}\right|>0$

$$
\begin{aligned}
g_{l}-\frac{4}{27}\left(\tilde{c}_{l} \xi\right)^{3}= & \left(d_{l}-\frac{4}{27}(-1)^{3(l+1)} c^{3}\right) b_{1}^{3} \xi^{3}+a_{0 l} f^{1 / 2} \xi^{2} \\
= & g_{l^{\prime}}+b_{l l^{\prime}}\left(d_{l} \xi^{2}\right)\left(\tilde{c}_{l} \xi\right)+\tilde{b}_{l l^{\prime}}\left(\tilde{c}_{l} \xi\right) \xi \\
& +\tilde{\tilde{b}}_{l l^{\prime}} c\left(\tilde{c}_{l} \xi\right)^{3}
\end{aligned}
$$

for some $b_{l l^{\prime}}, \tilde{b}_{l l^{\prime}} \in B_{h, T}$ if $l \neq l^{\prime}$. Therefore by using (3.31) and noting $\left|g_{l}\right| \geq$ $1 / 2 G_{l^{\prime}}$ on $\Gamma^{l, 3}\left(\varepsilon^{N_{0}}, \varepsilon\right)$ for $\varepsilon \in\left(0, \varepsilon_{0}\right)$ which follows from $G_{l^{\prime}}>1 / 10 G_{l}>\varepsilon^{-N \kappa_{0} / 10>2}$ on $\Gamma^{l, 3}\left(\varepsilon^{N_{0}}, \varepsilon\right)$ for $\varepsilon \in\left(0, \varepsilon_{0}\right)$,

$$
\begin{aligned}
& \left|g_{l}-\frac{4}{27}\left(\tilde{c}_{l} \xi\right)^{3}\right| H_{l}^{-1} \geq \varepsilon / 200-\left|b_{l l^{\prime}}\right|_{0, T}\left|d_{l} \xi^{2}\right| H_{l}^{-2 / 3} \\
& \quad-\left|\tilde{b}_{l l^{\prime}}\right|_{0, T}|\xi| H_{l}^{-2 / 3} \text { on } \Gamma^{l, 3}\left(\varepsilon^{N}, \varepsilon\right), \varepsilon \in\left(0, \varepsilon_{0}\right) .
\end{aligned}
$$

By (3.32) and the definition of $N_{0}$ there exists $C_{4}$ such that for any $\varepsilon \in\left(0, \varepsilon_{0}\right)$ the right hand side is estimated from below by $\varepsilon / 200-C_{4} \varepsilon^{2\left(\operatorname{deg} D_{3}+3\right)}$ on $\Gamma^{l, 3}\left(\varepsilon^{N_{0}}, \varepsilon\right)$ so that one can find $\varepsilon^{(2)} \in\left(0, \varepsilon_{0}\right)$ such that $\left|g_{l}-\frac{4}{27}\left(\tilde{c}_{l} \xi\right)^{3}\right| H_{l}^{-1} \geq \varepsilon / 210$ on $\Gamma^{l, 3}\left(\varepsilon^{N_{0}}, \varepsilon\right)$ for $\varepsilon \in\left(0, \varepsilon^{(2)}\right)$, and consequently by this estimate, (3.48), (3.30), (3.31), and (3.47) we can conclude that

$$
\begin{aligned}
& \left|D_{3}\left(z^{l 3}, w^{l 3}\right)\right| \geq \varepsilon^{2} / 200-C_{4}\left(1+\varepsilon^{-1}\right)^{\operatorname{deg} D_{3}-1} \varepsilon^{\operatorname{deg} D_{3}+3} \\
& \text { on } \Gamma^{l, 3}\left(\varepsilon^{N_{0}}, \varepsilon\right) \text { for } \varepsilon \in\left(0, \varepsilon^{(2)}\right) .
\end{aligned}
$$

Hence one can find $\varepsilon^{(3)} \in\left(0, \varepsilon^{(2)}\right)$ such that $\tilde{\Gamma}^{l, 3}(\varepsilon) \subset X_{3, \varepsilon}$ for $\varepsilon \in\left(0, \varepsilon^{(3)}\right)$ in view 
of (3.46) and the definition of $X_{3, \varepsilon}$. Finally we take $\varepsilon_{1}=\min \left(\varepsilon^{(1)}, \varepsilon^{(3)}\right)$, and $N_{0}$, $\varepsilon_{0}$, and $\varepsilon_{1}$ in the proof have the desired properties for $N_{0}, \varepsilon_{0}$, and $\varepsilon_{1}$ in the statement. This completes the proof of Lemma 3.4.

Set with the notations in Lemma 3.4,

$$
T_{0}=\left(\varepsilon_{1} / 2\right)^{N_{0}}, \Gamma_{l}=\Gamma_{l}\left(T_{0}\right), \Gamma^{l, i}=\Gamma^{l, i}\left(T_{0}, \varepsilon_{1} / 2\right) .
$$

Let $\chi(s) \in C^{\infty}(\boldsymbol{R})$ with $\chi=1$ when $s \geq 1, \chi=0$ when $s \leq 1 / 2,0<\chi<1$ when $1 / 2<$ $s<1$, and define the functions on $\boldsymbol{R}_{T_{0}}^{3}$ by

$$
\begin{aligned}
& \varphi^{l i}=\varphi_{l} \cdot \chi_{l i, \varepsilon_{1} / 2}, \varphi_{*}^{l i}=\varphi_{l}^{*} \cdot \chi_{l i, \varepsilon_{1}} \text { for } i=1,2, \\
& \varphi^{l 3}=\varphi_{l} \cdot \chi_{l 3, \varepsilon_{1} / 2}, \varphi_{*}^{l 3}=\varphi_{l}^{*} \cdot \chi_{l 3, \varepsilon_{1} / 4}, \\
& \tilde{\varphi}_{l}=(1-\chi)\left(160^{-1} t^{\kappa} G_{l}\langle\xi\rangle^{-3 / 2}\right), l=1,2
\end{aligned}
$$

where $\varphi_{l}=\Phi_{l, l, 80}, \varphi_{l}^{*}=\Phi_{l, 2 l, 40}$ with

$$
\begin{aligned}
& \Phi_{l, e, R}=\chi\left(e G_{l}, G_{l}^{-1}\right) \cdot \chi\left(R^{-1} t^{\kappa} G_{l}\langle\xi\rangle^{-3 / 2}\right), l \neq 1^{\prime}, e \in \boldsymbol{R}, \quad \text { and } \\
& \chi_{l 1, e}=\chi\left(e H_{l} G_{l}^{-1}\right), \chi_{l 2, e}=\chi\left(e G_{l} H_{l}^{-1}\right), \\
& \chi_{l 3, e}=\chi\left(e^{-1} H_{l}^{-1} G_{l}\right) \cdot \chi\left(e^{-1} H_{l} G_{l}^{-1}\right), e \in \boldsymbol{R} .
\end{aligned}
$$

Then we have

$$
\operatorname{supp} \varphi_{*}^{l i} \subset \Gamma^{l, i}, \varphi_{*}^{l i}=1 \text { on supp } \varphi^{l i}, \Sigma_{l}\left(\Sigma_{i} \varphi^{l i}+\tilde{\varphi}\right) \geq 1
$$

We shall explain (3.53). The second one would be easily observed. $\varphi_{l}, \varphi_{l}^{*}$, and $\tilde{\varphi}_{l}$ are as in the following:

$$
\begin{aligned}
& \tilde{\varphi}_{l}=1 \text { when } t^{\kappa} G_{l} \leq 80\langle\xi\rangle^{3 / 2}, \text { and supp } \tilde{\varphi}_{l} \subset\left\{t^{\kappa} G_{l} \leq 160\langle\xi\rangle^{3 / 2}\right\} ; \\
& \varphi_{1}=1 \text { when } t^{\kappa} G_{1} \geq 80\langle\xi\rangle^{3 / 2} \text { and } G_{2} \geq G_{1}, \text { and } \\
& \text { supp } \varphi_{1}^{*} \subset\left\{t^{\kappa} G_{1} \geq 20\langle\xi\rangle^{3 / 2}\right\} \cap \mathscr{D}^{1} ; \\
& \varphi_{2}=1 \text { when } t^{\kappa} G_{2} \geq 80\langle\xi\rangle^{3 / 2} \text { and } G_{2} \leq 2 G \text {, and supp } \varphi_{2}^{*} \\
& \quad \subset\left\{t^{\kappa} G_{2} \geq 20\langle\xi\rangle^{3 / 2}\right\} \cap \mathscr{D}^{2} \text {. }
\end{aligned}
$$

So, supp $\varphi_{l}^{*} \subset \Gamma_{l} \cap \mathscr{D}^{l}$ and $\sum_{l}\left(\varphi_{l}+\widetilde{\varphi}_{l}\right) \geq 1$ from which the rest of (3.53) follows, because supp $\chi_{l i, \varepsilon_{1}} \subset \mathscr{D}_{l}^{(i)}\left(\varepsilon_{1} / 2\right)$ for $i=1,2$ and supp $\chi_{l 3, \varepsilon_{1} / 4} \subset \mathscr{D}_{l}^{(3)}\left(\varepsilon_{1} / 2\right)$, and because $\sum_{i} \chi_{i, \varepsilon} \geq 1$. From the property of $\varepsilon_{0}$ in Lemma 3.4 we can choose $U_{i j}$, $\lambda_{i j k}, \varphi_{i j}, \varphi_{i j}^{*}$ as in Lemma 3.3 for $\eta=\varepsilon_{0}$ when $i=1,2$ and for $\eta=\varepsilon_{1} / 2$ when $i=3$. We define

$$
\begin{aligned}
& \Gamma^{l, i, j}=\left\{(t, x, \xi) \in \Gamma^{l, i} ;\left(z^{l i}, w^{l i}\right)(t, x, \xi) \in U_{i j}\right\}, \\
& \tilde{\Gamma}^{l, i, j}=\left\{(t, x, \xi) \in \Gamma^{l, i} ;\left(z^{l i}, w^{l i}\right)(t, x, \xi) \in\left(\operatorname{supp} \varphi_{i j}^{*}\right)^{c}\right\} .
\end{aligned}
$$


We regard $\Gamma^{l, i, j}$ and $\tilde{\Gamma}^{l, i, j}$ as subsets of $\boldsymbol{R}_{T_{0}}^{3}$. Then,

$$
\begin{aligned}
& \Gamma^{l, i}=\Gamma^{l, i, j} \cup \tilde{\Gamma}^{l, i, j}, \boldsymbol{R}_{T_{0}}^{3}=\Gamma^{l, i} \cup\left(\operatorname{supp} \varphi_{*}^{l i}\right)^{c}, \\
& \Gamma^{l, i, j} \text { and } \tilde{\Gamma}^{l, i, j} \text { are open in } \boldsymbol{R}_{T_{0}}^{3} .
\end{aligned}
$$

Now we define the functions on $\boldsymbol{R}_{T_{0}}^{3}, \varphi_{l i j}, \varphi_{l i j}^{*}, \sigma_{l i j k}, k=1,2,3$ by the following:

$$
\varphi_{l i j}, \varphi_{l i j}^{*}, \sigma_{l i j k}=0 \text { on }\left(\Gamma^{l, i, j}\right)^{c}
$$

and on $\Gamma^{l, i, j}$,

$$
\begin{aligned}
& \varphi_{l i j}=\varphi^{l i} \cdot \varphi_{i j}\left(z^{l i}, w^{l i}\right), \varphi_{l i j}^{*}=\varphi_{*}^{l j} \cdot \varphi_{i j}^{*}\left(z^{l i}, w^{l i}\right), \\
& \sigma_{1 l j 1}=H_{l}^{1 / 3} \cdot \varphi_{*}^{l 1} \cdot\left(\lambda_{l j 1}\left(z^{l 1}, w^{l 1}\right)+w_{1}^{l 1}\right) \cdot \varphi_{l j}^{*}\left(z^{l 1}, w^{l 1}\right), \\
& \sigma_{l 1 j k}=H_{l}^{1 / 3} \cdot \varphi_{*}^{l 1} \cdot \lambda_{1 j k}\left(z^{l 1}, w^{l 1}\right) \cdot \varphi_{1 j}^{*}\left(z^{l 1}, w^{l 1}\right) \text { for } k \neq 1, \\
& \sigma_{l 2 j k}=G_{l}^{1 / 3} \cdot \varphi_{*}^{l 2} \cdot \lambda_{2 j k}\left(z^{l 2}, w^{l 2}\right) \cdot \varphi_{2 j}^{*}\left(z^{l 2}, w^{l 2}\right), \\
& \sigma_{l 3 j k}=H_{l}^{1 / 3} \cdot \varphi_{*}^{l 3} \cdot \lambda_{3 j k}\left(z^{l 3}, w^{l 3}\right) \cdot \varphi_{3 j}^{*}\left(z^{l 3}, w^{l 3}\right) .
\end{aligned}
$$

Then $\varphi_{l i j}, \varphi_{l i j}^{*}, \sigma_{l i j k} \in C^{\infty}\left(\boldsymbol{R}_{T_{0}}^{3}\right)$ by (3.55), because they are $C^{\infty}$ in $\Gamma^{l, i, j}, \tilde{\Gamma}^{l, i, j}$, and (supp $\left.\varphi_{*}^{l i}\right)^{c}$, and we see from (3.53) and (3.40),

$$
\varphi_{i j}^{*}=1 \text { on } \operatorname{supp} \varphi_{l i j} \text {. }
$$

From (3.37), (3.38), (3.58), and (3.33) we obtain

$$
\begin{aligned}
& \tilde{q}(\sigma, \cdot)=\left(1+A_{0 l}\right)\left(\sigma-c_{l} \xi-\sigma_{l 1 j 1}\right) \prod_{k=2}^{3}\left(\sigma-\sigma_{l 1 j k}\right) \\
& \quad \text { if }(t, x, \xi) \in \operatorname{supp} \varphi_{l i j}
\end{aligned}
$$

where $c_{l}=\left(1+A_{0 l}\right)^{-1}(-1)^{l+1} c b_{l}$ as in (3.25), and

(3.60) $\tilde{q}(\sigma, \cdot)=\left(1+A_{0 l}\right) \prod_{k=1}^{3}\left(\sigma-\sigma_{l i j k}\right)$ if $(t, x, \xi) \in \operatorname{supp} \varphi_{l i j}$ for $i=2,3$.

From (3.40) and (3.42) $\sum_{j} \varphi_{i j}\left(z^{l i}, w^{l i}\right)=1$ on $\Gamma^{l, i}$ so that $\sum_{j} \varphi_{l i j}=\varphi^{l i}$ by (3.57), since $\operatorname{supp}\left(\Sigma_{j} \varphi_{l i j}\right) \subset \cup_{j} \operatorname{supp} \varphi_{l i j} \subset \Gamma^{l, i}$, and since supp $\varphi^{l i} \subset \Gamma^{l, i}$ by (3.53). Thus in view of the last of (3.53),

$$
\sum_{l=1}^{2}\left(\sum_{i=1}^{3} \Sigma_{j} \varphi_{l i j}+\tilde{\varphi}_{l}\right) \geq 1
$$

We shall consider the estimates of derivatives of the functions constructed above. To do so we prove the basic estimates first. We remark that since $\varepsilon_{0}^{1-N_{0} \kappa}>20, \varepsilon_{0}^{N}<\left\langle S_{0}, N_{0}\right\rangle 1, \kappa>1$ with the notation in the statement of Lemma 3.4, $\varepsilon^{1-N \kappa}>20$ and $\varepsilon^{N_{0}}<S_{0}$ for any $\varepsilon \in\left(0, \varepsilon_{0}\right)$ so that from (3.31) and (3.32),

$$
2^{-1} \leq\left|c b_{l} \xi\right| H_{l}^{-1 / 3} \leq 1 \text { on } \Gamma^{l, 1} \cup \Gamma^{l, 3},
$$




$$
\begin{aligned}
& 2^{-1} \leq\left|g_{l}\right| G_{l}^{-1} \leq 1 \text { on } \Gamma_{l}, \\
& \Gamma^{l, 1}=\Gamma^{l, 3}=\emptyset \text { if } c \equiv 0 ;
\end{aligned}
$$

there exists a constant $C>0$ such that

$$
\begin{aligned}
& \max \left(2, C^{-1} t^{-2 \kappa / 3}\right)<\langle\xi\rangle \text { on } \Gamma_{l}, \\
& C G_{l}^{2 / 3}>\left|d_{l}\right|\langle\xi\rangle^{2} \text { on } \Gamma_{l} .
\end{aligned}
$$

We also note that

$$
\begin{aligned}
& \frac{d^{k}}{d x^{k}} f(x)=\left(\delta^{2}-x^{2}\right)^{-2 k} \beta_{k}(x) f(x),|x|<\delta \quad \text { where } \beta_{k} \text { is a } \\
& \text { polynomial, }
\end{aligned}
$$

and that

(3.65) if $a \in E_{h, T}$,

$$
\partial^{\alpha} a(t, x)=t^{-\alpha_{1}} \cdot\left(\delta^{2}-x^{2}\right)^{-2 \alpha_{2}} \cdot\left(\sum_{j} \beta_{\alpha j}(x) a_{\alpha j}(t, x)\right) a(t, x),|x|<\delta
$$

with a finite number of $a_{\alpha j} \in B_{h, T}$ and polynomials $\beta_{\alpha j}$.

Lemma 3.5. We denote $\partial^{\alpha}=\partial_{t}^{\alpha} \partial_{x}^{\alpha} \partial_{\xi}^{\alpha}$ for $\alpha \in Z_{+}^{3}$. Then we have the followings.

$$
\begin{aligned}
& \left|\partial^{\alpha} a\right| \leq C_{\alpha} t^{1 / h \cdot \min \left(1, \alpha_{1}\right)-\alpha_{1}}, t \neq 0 \text { for } a \in B_{h, T} .
\end{aligned}
$$

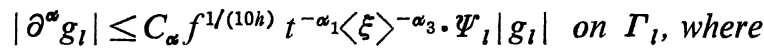

$$
\begin{aligned}
& \Psi_{l}=t^{1 /(6 h)}\langle\xi\rangle G_{l}^{-1 / 3} \\
& \left|\partial_{\xi}^{\alpha} g_{l}\right| \leq C_{k}\langle\xi\rangle^{-k}\left|g_{l}\right| \text { on } \Gamma_{l} \text { when } d_{l} \equiv 0 \text {. } \\
& \left|\partial^{\alpha}\left(d_{l} \xi^{3-i}\right)\right| \leq C_{\alpha}\left(t^{1 /(2 h)} f^{1 /(4 h)}\langle\xi\rangle G_{l}^{-1 / 3}\right)^{\min \left(3-i, \alpha_{2}\right)} \\
& \times t^{-\alpha_{1}}\langle\xi\rangle^{-\alpha_{3}} \cdot G_{l}^{(3-i) / 3} \text { on } \Gamma_{l} \text { for } i=1,2 \text {. } \\
& \left|\partial^{\alpha}(c \xi)\right| \leq C_{\alpha}\left(t^{1 /(2 h)} f^{1 /(4 h)}\langle\xi\rangle^{1 / 2} H_{l}^{-1 / 6}\right)^{\min \left(1, \alpha_{2}\right)} \\
& \times t^{-\alpha_{1}}\langle\xi\rangle^{-\alpha_{3}} \cdot H_{l}^{1 / 3} \text { on } \Gamma_{l} \text {. }
\end{aligned}
$$

We remark that from (3.62), $|x|<\delta$ on $\Gamma_{l}$, since $g_{l}=0$ when $|x| \geq \delta$.

Proof of Lemma 3.5. (3.65) is easy. We show (3.67). By (3.64), (3.65), and that $d_{l} \in E_{h, T}$,

$$
\begin{aligned}
\partial^{\alpha} g_{l}= & t^{-\alpha_{1}} \cdot\left(\delta^{2}-x^{2}\right)^{-2 \alpha_{2}} \cdot\left\{\left(\sum_{j} \beta_{\alpha j} a_{\alpha j}\right) g_{l}+\left(\sum_{j} \gamma_{\alpha j} b_{\alpha j}\right) f^{1 / 2} \xi^{2}\right\} \\
& \times \xi^{-\alpha_{3}} \text { if }|x|<\delta \text { and } \xi \neq 0
\end{aligned}
$$

with finite number of $a_{\alpha j}, b_{\alpha j} \in B_{h, T}$ and polynomials of $x, \beta_{\alpha j}, \gamma_{\alpha j}$. 
From (3.72),

$$
\begin{aligned}
& \left|\partial^{\alpha} g_{l}\right| \leq C_{\alpha^{\prime}} t^{-\alpha_{1}} \cdot\left(\delta^{2}-x^{2}\right)^{-2 \alpha_{2}}|\xi|^{-\alpha_{3}} \cdot\left(\left|g_{l}\right|+f^{1 / 2}|\xi|^{2}\right) \text { if }|x|<\delta \\
& \text { and } \xi \neq 0 \text {. }
\end{aligned}
$$

On the other hand,

$$
\begin{gathered}
\left|\delta^{2}-x^{2}\right|^{-k}\left|g_{l}\right|^{1 / 3} \leq C_{k} f^{1 /(10 h)}\left(t^{1 /(3 h)}|\xi|+|\xi|^{2 / 3}\right) \text { if }|x|<\delta ; \\
C^{-1} \Psi_{l} \leq\left(t^{1 /(6 h)}|\xi|+|\xi|^{2 / 3}\right) G_{l}^{-1 / 3}+|\xi|^{2} G_{l}^{-1}+1 \leq C \Psi_{l} \text { on } \Gamma_{b} .
\end{gathered}
$$

In fact, (3.74) follows easily from that $d_{l} \in E_{h, T}$, and the second inequality of (3.75) follows from the inequalities $\langle\xi\rangle^{-1 / 3}\left\langle C t^{2 \kappa / 9}\left\langle C t^{1 /(6 h)}\right.\right.$ on $\Gamma_{l}$ and $\langle\xi\rangle G_{l}^{-2 / 3}$ $<C t^{2 \kappa / 3}<C t^{1 /(6 h)}$ on $\Gamma_{l}$, both of which are consequences of (3.63) and that $2 \kappa / 9>1 /(6 h)$, and from which $G_{l}^{1 / 3} \leq\left(1+\left|g_{l}\right|\right)^{1 / 3} \leq C t^{1 /(6 h)}\langle\xi\rangle$. The first one of (3.75) is trivial. (3.67) is proved by combining (3.73) (3.75) and using the second fact of (3.62). (3.69) is trivially from that $|\xi|>1$ on $\Gamma_{l}$ by (3.63). We pass to (3.70). By (3.65) and the remark before proof,

$$
\left|\partial^{\alpha}\left(d_{l} \xi^{3-i}\right)\right| \leq C_{\alpha} t^{-\alpha_{1}} \cdot\left|\delta^{2}-x^{2}\right|^{-\alpha_{2}} \cdot\left|d_{l}\right||\xi|^{3-i-\alpha_{3}} \text { on } \Gamma_{l}
$$

from which (3.70) for $\alpha_{2}>3-i$ immediately follows, and when $\alpha_{2} \leq 3-i$,

$$
\begin{aligned}
& \left|\delta^{2}-x^{2}\right|^{-2 \alpha_{2}} \cdot\left|d_{l}\right| \cdot|\xi|^{3-i-\alpha_{3}} \\
& \quad \leq C\left(\left|\delta^{2}-x^{2}\right|^{-2 \alpha_{2}} \cdot\left|d_{l}\right|^{\alpha_{2} / 2}\right)\left|d_{l}\langle\xi\rangle^{2}\right|^{(3-i) / 2}\langle\xi\rangle^{-\alpha_{3}} \text { on } \Gamma_{l}
\end{aligned}
$$

from which (3.70) for $\alpha_{2} \leq 3-i$ follows immediately by using (3.63). (3.71) can be proved similarly as (3.70) by using that $c \in E_{h, T}$. The proof of Lemma 3.5 is complete.

Lemma 3.6. Let $U$ and $V$ be open sets of $\mathbb{R}_{y}^{m}$ and $\mathbb{R}_{x}^{n}$ respectively, let $F \in C^{\infty}(U)$, and let $\widetilde{F}=\left(\widetilde{F}_{1}, \cdots, \widetilde{F}_{n}\right)$ be $C^{\infty}$ mapping from $V$ into $U$. We assume that there exist positive functions $Z(y), N_{j}(y)$ on $U, j=1, \cdots, m$, and $M_{\alpha}(x)$ on $V, \alpha \in \mathbb{Z}_{+}^{n}$ satisfying $M_{\alpha+\beta}=M_{\alpha} \cdot M_{\beta}$ such that

$$
\sup _{y \in U}\left|\partial^{\alpha} F(y)\right| Z(y)^{-1} N(y)^{\alpha}<\infty \text { for all } \alpha \in \mathbb{Z}_{+}^{n}
$$

where $N=\left(N_{1}, \cdots, N_{m}\right)$ and $N^{\alpha}=\prod_{j=1}^{m} N_{j}^{\alpha}$, and such that

$$
\sup _{x \in V}\left|\partial^{\alpha} \widetilde{F}_{j}(x)\right| M_{\alpha}(x)^{-1} N_{j}(\tilde{F}(x))^{-1}<\infty \text { for all } \alpha \in \mathbb{Z}_{+}^{n} .
$$

Then, $\sup _{x \in V}\left|\partial^{\alpha}(F \circ \widetilde{F})(x)\right| Z(\widetilde{F}(x))^{-1} M_{\alpha}(x)^{-1}<\infty$ for all $\alpha \in \mathbb{Z}_{+}^{n}$.

This lemma is a simple consequence of the chain rule. We omit the details. 
Definition 3.7. Let $U$ be an open set of $\boldsymbol{R}^{n}$, and $Z(x) ; M_{\alpha x}(x), \alpha \in Z_{+}^{n}$ be positive functions on $U$ satisfying $M_{\alpha+\beta}=M_{\alpha} \cdot M_{\beta}$. Then we say that $a \in C^{\infty}(U)$ belongs to the set $\Xi\left(U, Z, M_{\alpha}\right)$ if

$$
\sup _{x \in \bar{U}}\left|\partial^{\alpha} a(x)\right| M_{\alpha}(x)^{-1} Z(x)^{-1}<\infty \text { for all } \alpha \in Z_{+}^{n} .
$$

Lemma 3.8. Let $U$ be an open set of $\boldsymbol{R}^{n}$. Then we have the followings.

1. If $a_{i} \in \Xi\left(U, Z_{i}, M_{\alpha}\right), i=1,2$, then $a_{1} a_{2} \in \Xi\left(U, Z_{1} \cdot Z_{2}, M_{\alpha}\right)$.

2. $\Xi\left(U, Z, M_{\alpha}\right) \subset \Xi\left(U, \tilde{Z}, \tilde{M}_{\alpha}\right)$ if $Z \leq \tilde{Z}$ and $M_{\alpha} \leq \tilde{M}_{\alpha}$ for alll $\alpha$.

3. Let $a=\left(a_{1}, \cdots, a_{m}\right)$ be $C^{\infty}$ mapping from $U$ into $\boldsymbol{R}^{m}$ (resp. $\left.(0,+\infty)^{m}\right)$ with $a_{i} \in \Xi\left(U, 1, M_{\alpha}\right)$ (resp. $a_{i} \in \Xi\left(U, a_{i}, M_{\alpha}\right)$ ), and let $F$ be $a C^{\infty}$ function on an open set of $\boldsymbol{R}^{m}$ (resp. $\left.(0,+\infty)^{m}\right)$ containing the image of a of $U$ such that $F \in$ $\Xi(V, Z, 1)$ (resp. $\Xi\left(V, Z,\left(y^{\alpha}\right)^{-1}\right)$. Then, $F \circ a \in \Xi\left(U, Z \circ a, M_{\alpha}\right)$.

4. Let $a=\left(a_{1}, \cdots, a_{m_{1}}, a_{m_{1}+1}, \cdots, a_{m}\right), m=m_{1}+m_{2}, m_{i} \geq 1$ be $C^{\infty}$ mapping from $U$ into $(0,+\infty)^{m}{ }_{1} \times \boldsymbol{R}^{m_{2}}$ with $a_{i} \in \Xi\left(U, a_{i}, M_{\alpha}\right)$ for $i=1, \cdots, m_{1}$ and $a_{i} \in \Xi\left(U, 1, M_{\alpha}\right)$ for $i=m_{1}+1, \cdots, m$, and $F$ be $a C^{\infty}$ function on an open set $V$ of $(0,+\infty)^{m_{1}} \times \boldsymbol{R}^{m_{2}}$ containing the image of a of $U$ such that $F \in \Xi\left(V, Z,\left(y^{\prime \alpha^{\prime}}\right)^{-1}\right)$ where $y^{\prime}=\left(y_{1}, \cdots\right.$, $\left.y_{m_{1}}\right)$ for $y \in \boldsymbol{R}^{m}$. Then, $F \circ a \in \Xi\left(U, Z \circ a, M_{\alpha}\right)$.

5. Let $a \in C^{\infty}(U)$, be positive, and $\in \Xi\left(U, a, M_{\alpha}\right)$. Then, $a^{s} \Xi\left(U, Z, M_{\alpha}\right)=$ $\Xi\left(U, a^{s} Z, M_{\alpha}\right)$ for all $s \in \boldsymbol{R}$.

Proof of Lemma 3.8. 1 follows from Leibniz rule, and 2 is trivial. The former (resp. latter) of 3 of the case that in Lemma 3.6, $V=U, U=V, Z=Z$, $\widetilde{F}=a, N_{j}=1$ (resp. $N_{j}=y_{j}$ ), $F=F$ where the right hand side denotes the notations in 3. 4 is similar to 3. To see 5 we take $F=t^{s}, t \in(0,+\infty), s \in \boldsymbol{R}$ in the latter of 3. Then, $a^{s} \in \Xi\left(U, a^{s}, M_{\alpha}\right)$ which implies from 1 that $a^{s} \Xi\left(U, Z, M_{\alpha}\right) \subset$ $\Xi\left(U, a^{s} Z, M_{\alpha}\right)$ and $a^{-s} \Xi\left(U, a^{s} Z, M_{\alpha}\right) \subset \Xi\left(U, Z, M_{\alpha}\right)$ as desired. The proof is complete.

Remark on notations. We denote a statement $*$ in a statement $* *$ by **-*. For example, 1 in Lemma 3.8 is denoted by Lemma 3.8-1.

We set for $l=1,2, \rho \in[0,1]$, an open set $U$ of $\boldsymbol{R}_{T_{0}}^{3}$, and a positive function $Z$ on $U$,

$$
\begin{aligned}
& \left.\Xi_{\rho}^{l}(U, Z) \text { (or simply, } \Xi_{\rho}^{l}(Z) \text { when } U=\boldsymbol{R}_{T_{0}}^{3}\right)=\Xi\left(U, Z, M_{\alpha}^{l, \rho}\right) \text { where } \\
& M_{\alpha^{\prime}}^{l, \rho}=t^{-\alpha_{1}}\left\langle f^{1 /(10 h)} \Psi_{l}>^{\alpha_{1}} \cdot \Psi_{l^{2}}^{\alpha^{2}+\alpha_{3}} \cdot\langle\xi\rangle^{-\alpha_{3}} .\right.
\end{aligned}
$$

We also define

$$
\rho_{l}=1 \text { when } d_{l} \neq 0, \rho_{l}=0 \text { when } d_{l} \equiv 0 \text {, for } l=1,2 \text {, and }
$$




$$
\rho_{0}=\max \left(\rho_{1}, \rho_{2}\right) \text {. }
$$

Fact 3.9. 1. $\left|\partial_{x, y}^{\alpha}\langle z\rangle^{s}\right| \leq C_{\alpha}\langle z\rangle^{s-|\alpha|}, z=x+\sqrt{-1} y$.

2. $\left|(d / d \xi)^{k} \varphi(\xi)\right| \leq C_{k}\langle\xi\rangle^{-k}$ if $\varphi \in C^{\infty}(\boldsymbol{R})$ and being constant if $|\xi| \geq R$ for some $R>0$.

3. $\left|(d / d t)^{k} t^{s}\right| \leq C_{s k} t^{s-k}, t>0, s \in \boldsymbol{R}$.

4. $\left|\partial_{t}^{s} \partial_{w}^{a}\left(\lambda_{1 j 1}+w_{1}\right)(t, w)\right| \leq C_{s a} t^{1-s}$ on $U_{1 j} \cap(0, \infty) \times C^{4}$.

5. $\left|\partial_{s}^{s} \partial_{w}^{\infty} \lambda_{1 j k}(t, w)\right| \leq C_{s \alpha} t^{3 / 2-s}$ on $U_{1 j} \cap(0, \infty) \times C^{4}$ for $k \neq 1$.

6. $\left|\partial_{z, w}^{a} \lambda_{i j k}(z, w)\right| \leq C_{a}$ on $U_{i j}$ for $i \neq 1$.

Fact 3.10. 1. $a \in \Xi_{0}^{l}(1)$ if $a \in B_{h, T}$, where we regard $a$ as a constant function in $\xi$. With similar convention,

2. $a \in \Xi_{0}^{l}\left(\langle\xi\rangle^{m}\right)$ if $a(x, \xi) \in S_{1,0}^{m}(\boldsymbol{R})$.

In view of Lemma 3.5 and that $\Psi_{l} \geq C_{1}>0$ on $\Gamma_{l}((3.75))$,

$$
\begin{aligned}
& g_{l} \in \Xi_{\rho_{l}}^{l}\left(\Gamma_{l}, G_{l}\right), c \xi \in \Xi_{0}^{l}\left(\Gamma^{l, 1} \cup \Gamma^{l, 3}, H_{l}^{1 / 3}\right), d_{l} \xi^{3-i} \in \Xi_{0}^{l}\left(\Gamma_{l}, G_{l}^{(3-i) / 3}\right) \\
& \text { for } i=1,2 .
\end{aligned}
$$

By noting Facts 3.11-1 and 3.9-1 an application of Lemma 3.8-1 to $c b_{l} \xi$ and that of Lemma 3.6 with $F=\langle z\rangle$ and (3.79) give

$$
G_{l}^{s} \in \Xi_{\rho_{l}}^{l}\left(\Gamma_{l}, G_{l}^{s}\right), H_{l}^{s} \in \Xi_{0}^{l}\left(\Gamma^{l, 1} \cup \Gamma^{l, 3}, H_{l}^{s}\right), s \in \boldsymbol{R} .
$$

In the similar way, applications of Lemma 3.9-1 to the last of (3.79), the former of (3.80), Fact 3.10-1, and those to Fact 3.10-1, 3.10-2, the former of (3.80) show that

$$
G_{l}^{-(3-i) / 3} \cdot v_{i} \in \Xi_{\rho_{l}}^{l}\left(\Gamma_{l}, 1\right),
$$

and by (3.80), (3.81), Lemma $3.8-2$,

$$
H_{l}^{-(3-i) / 3} \cdot v_{i} \in \Xi_{\rho_{l}}^{l}\left(\Gamma^{l, 1} \cup \Gamma^{l, 3},\left(G_{l} H_{l}^{-1}\right)^{(3-i) / 3}\right) .
$$

(3.71) shows that $\left|\partial^{\alpha}(c \xi)\right| \leq C_{\alpha} t^{1 /(2 h)-\alpha_{1}} \cdot f^{1 /(4 h)}\langle\xi\rangle^{1-\alpha_{3}}$ on $\Gamma_{l}$ when $\alpha_{2}>0$, and that $\left|\partial^{\alpha}(c \xi)\right| \leq C_{\alpha} M_{\alpha}^{l, 0} \cdot H_{l}^{1 / 3}$ on $\Gamma_{l}$ when $\alpha_{2}=0$. So, from that $M_{\alpha}^{l, \rho}$ is increasing respectively on $\alpha_{2}$ and $\rho$, from (3.80), and from the definition of $\Psi_{l}$, Leibniz rule gives

$$
c \xi \cdot G_{l}^{-1 / 3} \in \Xi_{\rho_{l}}^{l}\left(\Gamma^{l, 2}, 1\right) .
$$

Using (3.84), (3.79) (3.80), Fact 3.10-1, and Lemma $3.8-1,2$ it is easy to see that

$$
z^{l i}, w^{l i} \in \Xi_{\rho_{l}}^{l}\left(\Gamma^{l, i}, 1\right), z^{l 1} \in \Xi_{\rho_{l}}^{l}\left(\Gamma^{l, 1}, z^{l 1}\right)
$$


Applications of Lemma 3.8-4 with (3.84) and Fact 3.9-4,5, that of the former of Lemma 3.8-3 with (3.84) and Fact 3.9-6, and those of the former of Lemma 3.8-3 with (3.84) and that $\varphi_{i j}, \varphi_{i j}^{*} \in C_{0}^{\infty}\left(U_{i j}\right)$ give

$$
\begin{aligned}
& \left(\lambda_{l j 1}+w\right)\left(z^{l 1}, w^{l 1}\right) \in \Xi_{\rho_{l}}^{l}\left(\Gamma^{l, 1}, z^{l 1}\right), \\
& \lambda_{1 j k} \in \Xi_{\rho_{l}}^{l}\left(\Gamma^{l, 1},\left(z^{l 1}\right)^{3 / 2}\right) \text { for } k \neq 1, \\
& \lambda_{i j k}\left(z^{l i}, w^{l i}\right) \in \Xi_{\rho_{l}}^{l}\left(\Gamma^{l, i}, 1\right) \text { for } i \neq 1, \\
& \varphi_{i j}\left(z^{l i}, w^{l i}\right), \varphi_{i j}^{*}\left(z^{l i}, w^{l i}\right) \in \Xi_{\rho_{l}}^{l}\left(\Gamma^{l, 1}, 1\right) .
\end{aligned}
$$

Lemma 3.11. Let $U$ and $V$ be open sets of $\boldsymbol{R}^{n}$ with $U \subset V$, and let $Z, \tilde{Z}, M_{\alpha}$ with $\alpha \in Z_{+}^{n}$ be positive functions on $V$ satisfying $M_{\alpha+\beta}=M_{\alpha} \cdot M_{\beta}$. Then we have the followings.

1. $\partial^{\beta} a \in \Xi\left(V, M_{\beta} Z, M_{\alpha}\right)$ if $a \in \Xi\left(V, Z, M_{\alpha}\right)$.

2. Let $a \in C^{\infty}(V)$ and assume that supp $d a \subset U$, that $\left.a\right|_{U} \in \Xi\left(U,\left.Z\right|_{U},\left.M_{\alpha}\right|_{U}\right)$, and that $|a| \leq C Z$ for some constant $C$. Then $a \in \Xi\left(V, Z, M_{\alpha}\right)$.

3. Let $a, b \in C^{\infty}(V)$ and assume that $a \in \Xi\left(V, Z, M_{\alpha}\right)$, that supp $a \cap \operatorname{supp} d b \subset$ $U$, that $\left.b\right|_{U} \in \Xi\left(U,\left.\tilde{z}\right|_{U},\left.M_{\alpha}\right|_{U}\right)$, and that $|b| \leq C Z$ for some constant $C$. Then $a \cdot b \in \Xi\left(V, Z \cdot \tilde{Z}, M_{\alpha}\right)$.

1 and 2 are obvious. 3 is a simple consequence of Leibniz rule. We omit the details.

Lemma 3.12. Let $l \neq l^{\prime}$, and define functions on $\boldsymbol{R}_{T_{0}}^{3}$ by

$$
a_{1}=G_{l^{\prime}} \cdot G_{l}^{-1}, a_{2}=t^{\kappa} G_{l}\langle\xi\rangle^{-3 / 2}, a_{3}=G_{l} H_{l}^{-1} .
$$

Let $\chi_{1}(s) \in C^{\infty}(\boldsymbol{R})$ supported in $[1 / 2, \infty)$ and $=1$ on $[1, \infty)$, and set

$$
\begin{aligned}
& b_{0}=\chi_{1}\left(e a_{1}\right) \cdot \chi_{1}\left(R^{-1} a_{2}\right), b_{1}=\chi_{1}\left(\varepsilon a_{3}^{-1}\right), b_{2}=\chi_{1}\left(\varepsilon a_{3}\right), \\
& b_{3}=\chi_{1}\left(\varepsilon^{-1} a_{3}\right) \cdot \chi_{1}\left(\varepsilon^{-1} a_{3}\right) \text { for } e \in(0,5), R \in(20, \infty), \varepsilon \in\left[\varepsilon_{1} / 4, \varepsilon_{1}\right]
\end{aligned}
$$

where $\varepsilon_{1}$ is the constant in (3.51).

Then we have that

$$
\begin{aligned}
& \operatorname{supp} b_{0} \subset \Gamma_{1} \cap \Gamma_{2} \cap \mathscr{D}^{1}, b_{0} \in \Xi_{\rho_{0}}^{1}(1), \\
& b \cdot b_{i} \in \Xi_{\rho_{0}}^{l}(1) \text { if } b \in \Xi_{\rho_{0}}^{l}(1) \quad \text { with supp } \quad b \subset \Gamma_{l} \cap \mathscr{D}^{l}, i=1,2,3 .
\end{aligned}
$$

Proof of Lemma 3.12. We note that

$$
a_{1} \in \Xi_{\rho_{0}}^{l}\left(\Gamma_{1} \cap \Gamma_{2} \cap \mathscr{D}^{l}, a_{1}\right), a_{2} \in \Xi_{\rho_{l}}^{l}\left(\Gamma_{l}, a_{2}\right), a_{3} \in \Xi_{\rho_{l}}^{l}\left(\Gamma^{l, 1} \cap \Gamma^{l, 3}, a_{3}\right) .
$$

Indeed, the latter two are clear from (3.80) and the first one follows from (3.80), Lemma 3.8-1, the fact that 


$$
G_{l^{\prime}} \in \Xi_{\rho^{\prime}}^{l}\left(\Gamma_{l^{\prime}} \cap \mathscr{D}^{l}, G_{l^{\prime}}\right)
$$

which follows from Lemma 3.8-1, the fact that $\Psi_{l^{\prime}} \leq 10^{1 / 3} \Psi_{l}$ on $\mathscr{D}^{l}$ which

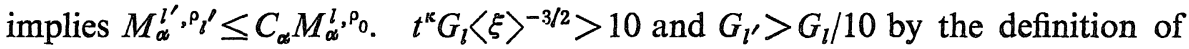
$\chi_{1}$ and the range of $e, R$, which implies the first one of (3.86). So, the former two of (3.87) and an application of the latter of Lemma 3.8-3 with $F(y)=$ $\chi_{1}\left(e y_{1}\right) \cdot \chi_{1}\left(R^{-1} y_{2}\right)$ yields $b_{0} \in \Xi_{\rho_{0}}^{l}\left(\Gamma_{1} \cap \Gamma_{2} \cap \mathscr{D}^{l}, 1\right)$ so that consequently the first one of (3.86) and Lemma 3.11-2 shows $b_{0} \in \Xi_{\rho_{0}}^{l}(1)$. To see the last one, we observe from (3.30), the range of $\varepsilon$, and the definition of $\chi_{1}$ that

$$
\mathscr{D}^{l} \cap \Gamma_{l} \cap \operatorname{supp} d b_{i} \subset \Gamma^{l, 3}, i=1,2,3 \text {. }
$$

In fact,

$$
\begin{aligned}
& \text { supp } d b_{1} \subset\left\{(t, x, \xi) \in \boldsymbol{R}_{T_{0}}^{3} ; a_{3}(t, x, \xi) \in[\varepsilon, 2 \varepsilon]\right\}, \\
& \text { supp } d b_{2} \subset\left\{a_{3} \in[1 /(2 \varepsilon), 1 / \varepsilon]\right\}, \operatorname{supp} b_{3} \subset\left\{a_{3} \in[\varepsilon / 2,2 / \varepsilon]\right\}, \\
& \mathscr{D}_{1}^{(3)} \cap \boldsymbol{R}_{T_{0}}^{3}=\left\{a_{3} \in(\varepsilon / 10,10 / \varepsilon)\right\},
\end{aligned}
$$

so that $U_{i} \operatorname{supp} d b_{i} \subset \mathscr{D}_{l}^{(3)}\left(\varepsilon_{1} / 2\right)$ which implies (3.88). Since the latter of Lemma 3.8-3 yields $b_{i} \in \Xi_{\mathrm{P}_{l}}^{l}\left(\Gamma^{l, 3}, 1\right)$ from the last one of (3.86) and the definition of $b_{i}$, an application of Lemma 3.11-3 with $U=\Gamma^{l, 3}, V=\mathbb{R}_{T_{0}}^{3}, b=b_{\dot{z}}, a=b$, where the notations in the proof are on the right, gives the last one of (3.86) in view of supp $b \subset \Gamma_{l} \cap \mathscr{D}^{l}$. The proof is complete.

Lemma 3.12 yields from the definitions (3.51), (3.52),

$$
\varphi^{l i}, \varphi_{*}^{l i} \in \Xi_{\rho_{0}}^{l}(1)
$$

and similarly an application of the latter of Lemma 3.8-3 with $F=\chi$ in (3.51), $U=\Gamma_{l}, a=a_{2}$ in Lemma 3.12 gives from (3.86) and Fact 3.9-2 that $\tilde{\varphi}_{l} \in \Xi_{\rho_{l}}^{l}\left(\Gamma_{l}, 1\right)$ so that from the fact that $d \widetilde{\varphi}_{l} \subset \Gamma_{l}$ which follows from the definition of $\tilde{\varphi}_{l}$ and that $d \tilde{\chi}=0$ on $(-\infty, 1 / 2]$, Lemma $3.11-2$ and the boundedness of $\tilde{\varphi}_{l}$ yield that

$$
\tilde{\varphi}_{l} \in \Xi_{\rho_{l}}^{l}(1) \text { and supp } d \tilde{\varphi}_{l} \subset \Gamma_{l} .
$$

From the definition (3.57), and (3.85), (3.80), (3.89), Lemma 3.11-2 yield by taking $U=\Gamma^{l, i, j}$,

$$
\begin{gathered}
\varphi_{l i j}, \varphi_{l i j}^{*} \in \Xi_{\rho_{l}}^{l}(1), \\
\sigma_{l 1 j 1} \in \Xi_{\rho_{0}}^{l}\left(G_{l}^{1 / 3}\right), \sigma_{l 1 j k} \in \Xi_{\rho_{0}}^{l}\left(G_{l}^{1 / 2} H_{l}^{-1 / 6}\right) \text { for } k \neq 1, \\
\sigma_{l 2 j k} \in \Xi_{\rho_{0}}^{l}\left(G_{l}^{1 / 3}\right), \sigma_{l 3 j k} \in \Xi_{\rho_{0}}^{l}\left(H_{l}^{1 / 3}\right) .
\end{gathered}
$$

To define $\varphi_{i}^{*}$ as in (3.28) later, in the similar way as (3.51), (3.52), (3.57) we 
set with the notations in Lemma 3.12,

$$
\varphi_{l j}^{*}=\left[\begin{array}{ccc}
\varphi_{l}^{*} \cdot \varphi_{j}^{*}\left(z^{l 2}, w^{l 2}\right) & \text { on } \Gamma^{l, 2, j} \\
0 & \text { on }\left(\Gamma^{l, 2, \jmath}\right)^{c}
\end{array}\right.
$$

where $\varphi_{l}^{*}=\tilde{\chi}\left(2 l a_{1}\right) \cdot \tilde{\chi}\left(40^{-1} a_{2}\right) \cdot \tilde{\chi}\left(\varepsilon_{1} a_{3}\right)$ with $\tilde{\chi} \in C^{\infty}(\boldsymbol{R})$ supported in $[2 / 3, \infty)$ and $=1$ on $[3 / 4, \infty)$, and $\varphi_{j}^{*} \in C_{0}^{\infty}\left(U_{2 j}\right)$ such that $\varphi_{j}^{*}=1$ on $\operatorname{supp} \varphi_{2 j}$ and $\inf _{\operatorname{supp} \varphi_{j}^{*}} \varphi_{2 j}^{*}>0$.

The existence of such $\varphi_{j}^{*}$ is assured by the fact that $\varphi_{2 j}^{*}=1$ on $\operatorname{supp} \varphi_{2 j}$.

Since for the $\chi$ in (3.51) we have that inf $\chi>0$, and since the function $\operatorname{supp} \tilde{x}$ $\tilde{\chi}(2 s)$ equals to 1 on supp $\chi$, we see from (3.93), (3.51), (3.52), (3.57) that

$$
\begin{aligned}
& \varphi_{l j}^{*}=1 \text { on supp } \varphi_{l 2 j}, \text { and there exists } C>0 \text { such that } \\
& \varphi_{l 2 j}^{*} \geq C \text { on supp } \varphi_{l j}^{*} .
\end{aligned}
$$

As the derivation of (3.89) Lemma 3.8-3, (3.84), Lemma 3.12, and Lemma 3.11-2 show that

$$
\varphi_{l j}^{*} \in \Xi_{\rho_{0}}^{l}(1) .
$$

Now the defintion of $\Gamma_{l}$ gives with the notations in Lemma 3.1

$$
\Psi_{l}=t^{1 /(6 h)}\langle\xi\rangle G_{l}^{-1 / 3} \leq t^{1 /(6 h)+\kappa / 3}\langle\xi\rangle^{1 / 2} \leq t^{1 /(18 h)-1 /(20 h)} \psi_{2}^{-1} \text { on } \Gamma_{l} .
$$

This easily implies that

$$
t^{-1}\left\langle f^{1 /(10 h)} \Psi_{l}\right\rangle \leq 2 \psi_{1}^{-1} \text { on } \Gamma_{l}
$$

so that

$$
M_{\alpha}^{l, \rho} \leq 2^{\alpha}{ }_{1} \psi_{\rho / 2, \alpha} \text { on } \Gamma_{l} \text {, where } \psi_{\rho / 2, \alpha}=\psi_{1}^{-1} \cdot \psi_{2}^{-\alpha_{2}} \cdot \psi_{3, \rho / 2}{ }^{-\alpha_{3}} \text {. }
$$

Thus, since $\Psi_{l} \geq C>0$ on $\Gamma_{l}$ means $G_{l}^{1 / 3} \leq C^{-1} t^{1 /(6 h)}\langle\xi\rangle$ on $\Gamma_{l}$, since the definition of $\Gamma^{l, i}$ and (3.92) imply $\sigma_{l i j k} \in \Xi_{\rho_{0}}^{l}\left(G_{l}^{1 / 3}\right)$, and since supp $\sigma_{l i j k}$, supp $\varphi_{l i j}$, supp $\varphi_{l i j}^{*}$, supp $\varphi_{l j}^{*}$, supp $d \widetilde{\varphi}_{l}$ are all contained in $\Gamma_{l}$, we have from (3.90) (3.92), (3.95), Lemma 3.8-2, Lemma 3.11-2 that $\varphi_{l i j}, \varphi_{i j}^{*}, \varphi_{l j}^{*}, \widetilde{\varphi}_{l} \in \boldsymbol{\Xi}\left(\boldsymbol{R}_{T_{0}}^{3}, 1\right.$, $\left.\psi_{\rho_{0} / 2, \alpha}\right)$ and $\sigma_{l i j k} \in \Xi\left(\boldsymbol{R}_{T_{0}}^{3}, t^{1 /(6 h)}\langle\xi\rangle, \psi_{\rho_{0} / 2, \alpha}\right)$. By the latter of this, Lemma 3.8-5, Fact 3.9-3, we obtain $\sigma_{l i j k} \in t^{1 /(6 h)} \Xi\left(\boldsymbol{R}_{T_{0}}^{3},\langle\xi\rangle, \psi_{\rho_{0 / 2}, \alpha}\right)$. Therefore, noting that

$$
\left(\psi_{2}\right)^{-\rho} \psi_{3, \rho / 2}=\langle\xi\rangle,
$$

we obtain with $T=T_{0}$ and the given $h$

$$
\sigma_{l i j k} \in t^{1 /(6 h)} \Sigma_{\rho_{0} / 2}^{\left(0,-\rho_{0}, 1\right)}, \varphi_{l i j}, \varphi_{l i j}^{*}, \varphi_{l j}^{*} \in \Sigma_{\rho_{0} / 2}^{0} .
$$


Similarly from Lemmas $3.11-1,3.8-2$ and that

$$
\begin{aligned}
& G_{l}^{1 / 3} \Psi_{l}=t^{1 /(6 h)}\langle\xi\rangle, G_{l}^{1 / 3} t^{-1}\left\langle f^{1 /(10 h)} \Psi_{l}\right\rangle \leq 2 t^{1 /(6 h)-1}\langle\xi\rangle, \\
& G_{l}^{1 / 3}\langle\xi\rangle^{-1}=t^{1 /(6 h)} \Psi_{l}^{-1},
\end{aligned}
$$

we have

$$
\begin{aligned}
& \partial_{t} \sigma_{l i j k} \in t^{1 /(6 h)-1} \sum_{\rho_{0} / 2}^{\left(0,-\rho_{0}, 1\right)}, \partial_{x} \sigma_{l i j k} \in t^{1 /(6 h)} \sum_{\rho_{0} / 2}^{\left(0,-\rho_{0}, 1\right)}, \\
& \partial_{\xi} \sigma_{l i j k} \in t^{1 /(6 h)} \sum_{\rho_{0} / 2}^{\left(0,1-\rho_{0}, 0\right)} .
\end{aligned}
$$

If $d_{l_{1}} \equiv 0$ which implies $G_{l_{1}} \leq C\langle\xi\rangle^{2}$, and if $l_{2} \neq l_{1}$, the definitions of $\mathscr{D}^{l_{2}}$ and $\Gamma^{l, i}$ show that $G_{l_{2}} \leq C^{\prime}\langle\xi\rangle^{2}$ on $\cup_{i=1}^{3} \Gamma^{l^{i}}$ and $H_{l} \leq C^{\prime}\langle\xi\rangle^{2}$ for $\Gamma^{l, 2} \cup \Gamma_{l}^{, 3}$ for any $l$. Thus, since $\sigma_{l i j k} \in \Xi_{\rho_{0}}^{l}\left(G_{l}^{1 / 3}\right)$ for any $l$, we have that

$$
\begin{gathered}
\sigma_{l i j k} \in \Sigma^{2 / 3\left(0,-\rho_{0}, 1\right)}, \\
\left|g_{l}\right| \leq C\langle\xi\rangle^{2} \text { on } \cup_{i=1}^{3} \Gamma^{l, i}, \\
|c| \leq C\langle\xi\rangle^{-1 / 3} \text { on } \cup_{l}\left(\Gamma^{l, 2} \cup \Gamma^{l, 3}\right),
\end{gathered}
$$

if either $d_{1}$ or $d_{2}$ is identically zero.

Moreover, since (3.79), (3.92), Lemma 3.8-1 imply that $c \xi \cdot \sigma_{l 1 j k} \in \Xi_{\rho_{0}}^{l}\left(\Gamma^{l, 1}\right.$, $\left.G_{l}^{1 / 2} H_{l}^{1 / 6}\right)$ for $k \neq 1$, and since $G_{l}^{1 / 2} H_{l}^{1 / 6} \leq C\langle\xi\rangle^{3 / 2}$ on $\Gamma^{l, 1}$ if $d_{1} d_{2} \equiv 0$, by Lemmas $3.11-3,3.8-2$ we have that

$$
c \xi \cdot \sigma_{l 1 j k} \in \Sigma_{\rho_{0} / 2}^{3 / 2\left(0,-\rho_{0}, 1\right)} \text { for } k \neq 1 \text { if } d_{1} d_{2} \equiv 0 .
$$

From (3.62) we have that

$$
\varphi_{l i j} \equiv 0 \text { for } i=1,3 \text { if } c \equiv 0 \text {. }
$$

From the fact that $\operatorname{supp} \varphi_{l i j}^{*} \subset \Gamma^{l, i, j},(3.44),(3.39)$, and the definition of $\sigma_{l i j k}$,

$$
C\left|\sigma_{l 2 j k}-\sigma_{l 2 j k^{\prime}}\right| \geq \max _{s}\left|\sigma_{l 2 j s}\right|+G_{l}^{1 / 3} \text { on supp } \varphi_{l j}^{*} \text { if } k \neq k .
$$

End of the proof of Lemma 3.2. We may assume that the set of suffixes of $\left\{U_{i j}\right\}_{j}$ is $\{1, \cdots, J(i)\}$ for some $J(i) \in N$. We numberize the elements of the set $\Lambda=\{(l, i, j) ; l=1,2, i=1,2,3, j=1, \cdots, J(i)\}$ so that $\Lambda=\left\{\nu(s) ; s=1, \cdots, s_{0}\right\}$, $s_{0}=\psi(\Lambda)$, and denote $\varphi_{l i j}$ by $\varphi_{\nu(s)}, \sigma_{l i j k}$ by $\sigma_{\nu(s), k}$ if $\nu(s)=(l, i, j)$. We define

$$
\Phi=\Sigma_{s} \varphi_{v(s)}+\Sigma_{l} \tilde{\varphi}_{l} .
$$

Then since $\Phi \in \Sigma_{\rho_{0} / 2}^{0}, \Phi \geq 1$, we have that $\Phi \in \Xi\left(\boldsymbol{R}_{T_{0}}^{3}, \Phi, \psi_{\rho_{0} / 2, \alpha}\right)$ so that $\Phi^{-1} \in \sum_{\rho_{0 / 2}}^{0}$ by Lemma 3.8-3. We shall define $\varphi_{i}, \sigma_{i j}, \varphi_{0 l}, \varphi_{0}$ in the statement, Case (1). Set $I=\left\{1, \cdots, s_{0}\right\}$ and $\varphi_{0 l}=\tilde{\varphi}_{l} \Phi^{-1}$ for $l=1,2, \varphi_{s}=\varphi_{v(s)} \Phi^{-1}, \sigma_{s k}=\sigma_{v(s) k}$ for $s \in I$. 
Case (2). Set $I=\left\{1, \cdots, s_{0}\right\}, \Lambda_{1}=\{(l, i, j) \in \Lambda, i \neq 1\}, \Lambda_{2}=\Lambda \backslash \Lambda_{1}, I_{i}=\{s \in I$; $\left.\nu(s) \in \Lambda_{i}\right\}$. The definitions of $\varphi_{i}, \sigma_{i j}, \varphi_{0 l}$ are the same as in case (1).

Case (3). Taking account of (3.102), (3.15) we set $\Lambda_{3}=\{(l, i, j) \in \Lambda ; i=2\}(\neq \phi)$. With a numbering $\Lambda_{3}=\left\{\nu^{\prime}(s) ; s=1, \cdots, s_{3}\right\}$ and the definition $I=\left\{1, \cdots, s_{3}\right\}$ we set $\varphi_{s}=\varphi_{\nu^{\prime}(s)} \Phi^{-1}, \varphi_{s}^{*}=\varphi_{l j}^{*}$, where $\nu^{\prime}(s)=(l, 2, j)$, and $\sigma_{s k}=\sigma_{\nu^{\prime}(s) k}$ for $s \in I, \varphi_{0}=$ $\left(\tilde{\varphi}_{1}+\tilde{\varphi}_{2}\right) \Phi^{-1}$. Note that $\Sigma_{s} \varphi_{s}+\varphi_{0} \geq 1$ from (3.102).

Then the conclusion of Lemma 3.2 follows from (3.14), (3.15), (3.29), (3.51), (3.56), (3.59), (3.60), (3.62), (3.63), (3.78), (3.93), (3.94), (3.97) (3.101), (3.103). The proof is complete.

\section{§4. Pseudodifferential Operators Used in the Proof of Proposition 2.3}

Let $S_{\rho, \theta}^{m}(\boldsymbol{R}), 1 \geq \rho \geq \theta \geq 0, \theta<1$ be the usual symbol class i.e. the set of all $a(x, \xi) \in C^{\infty}\left(\boldsymbol{R}^{2}\right)$ satisfying for any $\alpha, \beta$

$$
\left|a_{(\alpha)}^{(\beta)}(x, \xi)\right| \leq C_{\alpha \beta}\langle\xi\rangle^{m+\theta \alpha-\rho \beta} \quad \text { with some constant } C_{\alpha \beta}
$$

where $a_{(\alpha)}^{(\beta)}=\partial_{\xi}^{\beta} \partial_{x}^{\alpha} a$ which is a Frechet space with the topology defined by seminorms

$$
|a|_{l}^{(m)}=\max _{|\alpha|+|\beta| \leq l} \sup _{x, \xi}\left|a_{(\alpha)}^{(\beta)}\right|\langle\xi\rangle^{-m-\theta \alpha+\rho \beta} .
$$

We recall the definition (2.2) of the function $f$. We define ${ }^{f} a \in C^{\infty}\left(\boldsymbol{R}^{2}\right)$ for $a \in C^{\infty}\left(\boldsymbol{R}^{2}\right)$ by

$$
{ }^{f} a(x, \xi)=a(x, f(x) \xi) .
$$

We denote $L^{2}(\boldsymbol{R})$ norm by \|\| and the symbol in $S_{p, \theta}^{2 m}(\boldsymbol{R})$ of $a(x, D) b(x, D)$ for $a, b \in S_{\boldsymbol{p}, \theta}^{m}(\boldsymbol{R})$ with some $\rho, \theta, m$ by $a \circ b$. Then we prove an interpolation lemma.

Lemma 4.1. (1) Suppose $m^{\prime} \geq m$ and let $B$ be a bounded set of $S_{1,0}^{m}(\boldsymbol{R})$ and $b \in S_{1,0}^{m^{\prime}}(\boldsymbol{R})$ with $|b| \geq C_{0}\langle\xi\rangle^{m^{\prime}}$ when $|\xi| \geq R$ for some $C_{0}>0, R>0$. Then there exists $C>0$ such that

$$
\begin{gathered}
\|f a(x, D) u\| \leq C\left(\varepsilon^{m^{\prime}-m}\left\|{ }^{f} b(x, D) u\right\|+\varepsilon^{-\max (m, 0)}\|u\|\right), \\
\text { for } a \in B, u \in \mathcal{S}(\boldsymbol{R}), \varepsilon \in(0,1] .
\end{gathered}
$$

(2) Let $m^{\prime}, m, b$ as in (1) and $a \in S_{1, \theta}^{m}(\boldsymbol{R})$ with $\theta>0$. Then there exists $C>0$ such that

$$
\left\|f^{\max (m, 0)} a(x, D) u\right\| \leq C\left(\varepsilon^{m^{\prime}-m}\left\|\Lambda_{m^{\prime}} u\right\|+\varepsilon^{-\max (m, 0)}\|u\|\right),
$$




$$
\begin{aligned}
& \text { for } u \in \mathcal{S}(\boldsymbol{R}), \varepsilon \in(0,1] \text {, where } \\
& \Lambda_{s}=\left(\langle f(x) \xi\rangle^{s}\right)(x, D) \text { for } s \in \boldsymbol{R} .
\end{aligned}
$$

Proof. (1) Let $\chi \in C^{\infty}(\boldsymbol{R})$ with $\chi=1$ on $[2 R, \infty)$ and $\chi=0$ 'on $(-\infty, R]$. For $a \in B$ and $b$ we set $b_{\varepsilon}=\varepsilon^{m^{\prime}-m} b \chi, c_{\varepsilon}=\varepsilon^{-\max (m, 0)} b /|b| \chi, a_{1}=a \cdot \chi\left(2^{-1} \bullet\right)$, $a_{0}=a-a_{1}, s_{\varepsilon}=a_{1} / \varphi_{\varepsilon}$ for $|\xi| \geq 2 R$ and $=0$ for $|\xi|<2 R$ where $\varphi_{\varepsilon}=\varepsilon^{m^{\prime-m}} b+$ $\varepsilon^{-\max (m, 0)} b /|b|$ for $|\xi|>R$. Then $\left\{a_{1}\right\}_{a \in B},\left\{a_{0}\right\}_{a \in B}$ are bounded in $S_{1,0}^{m}$ and $S_{1,0}^{0}$ respectively, and $\varepsilon^{\max (m, 0)} c_{\varepsilon}$ is bounded in $S_{1,0}^{0}$ in $\varepsilon$ by the assumption on $b$. From the assumption on $b$ it is easy to see that there exists $C_{\alpha \beta}>0$ for any $\alpha, \beta$ such that

$$
\begin{aligned}
& \left|s_{\varepsilon(\alpha)}^{(\beta)}\right| \leq C_{\alpha \beta} \varphi_{\varepsilon}^{-1}\langle\xi\rangle^{m-\beta} \text { for }|\xi|>R \text { and any } a \in B, \\
& \left|b_{\varepsilon(\alpha)}^{(\beta)}\right| \leq C_{\alpha \beta} \varepsilon^{m^{\prime}-m}|b|\langle\xi\rangle^{-\beta} \leq C_{\alpha \beta} \varphi_{\varepsilon}\langle\xi\rangle^{-\beta} \text { for }|\xi|>R,
\end{aligned}
$$

which imply that $\varepsilon^{m-m^{\prime}} b_{\varepsilon}$ is bounded in $S_{1,0}^{m^{\prime}}$ in $\varepsilon$ and that $S_{\varepsilon}$ is bounded in $S_{1,0}^{0}$ in $\varepsilon$ from the inequality $\langle\xi\rangle^{m} \leq C\left|\varphi_{\varepsilon}(x, \xi)\right|$ when $|\xi|>R$. We note that $a=s_{\varepsilon} b_{\varepsilon}+s_{\varepsilon} c_{\varepsilon}+a_{0}$. Then we need the followings:

(4.3) There exists $C_{\alpha \beta}$ for any $\theta \in(0,1)$ such that for any $e \in S_{1,0}^{k}$

$$
\left|\left({ }^{f} e\right)_{(\alpha)}^{(\beta)}\right| \leq C_{\alpha \beta}|e|_{\alpha|+| \beta \mid}^{(k)}\langle f(x) \xi\rangle^{k-\theta \alpha}\langle\xi\rangle^{\theta \alpha-\beta} \text {. }
$$

$$
\begin{aligned}
& \text { For any } e \in C^{\infty}\left(\mathbb{R}^{2}\right), \\
& \begin{aligned}
\left({ }^{f} e\right)_{(\alpha)}^{(\beta)}(x, \xi)= & \sum_{\left(\alpha^{\prime}, \beta^{\prime}\right) \in D}\left(\delta^{2}-x^{2}\right)^{-2\left(\alpha-\alpha^{\prime}\right)} \psi_{\alpha^{\prime}, \beta^{\prime}}(x) f^{\beta}(x)(f(x) \xi)^{\left(\beta^{\prime}-\beta\right)} \\
& \times^{f}\left(e_{\left(\beta^{\prime}\right)}^{\left(\alpha^{\prime}\right)}\right)(x, \xi),|x|<\delta
\end{aligned}
\end{aligned}
$$

where $D_{\alpha, \beta}=\left\{\left(\alpha^{\prime}, \beta^{\prime}\right) \in \mathbb{Z}_{+}^{2} ; \alpha \geq \alpha^{\prime}, \beta^{\prime} \geq \beta, \alpha^{\prime}+\beta^{\prime}-\beta \leq \alpha, \beta^{\prime} \geq \min \left(1, \alpha-\alpha^{\prime}\right)\right\}$ and $\psi_{\alpha^{\prime}, \beta^{\prime}}$ are polynomials depending only on $\alpha, \beta, \alpha^{\prime}, \beta^{\prime}, f$ such that $\psi_{\alpha^{\prime}, \beta^{\prime}}=1$ when $\alpha=\alpha^{\prime}$ (which implies $\beta=\beta^{\prime}$ ).

(4.4) is proved by induction on $\alpha$ and (4.3) is a simple consequence of chain rule and inequality that

$$
\left|(f(x) \xi)_{(\alpha)}^{(\beta)}\right| \leq C_{\alpha \beta \theta}\left(\langle\xi\rangle\langle\langle f(x) \xi\rangle)^{\theta \alpha}\langle f(x) \xi\rangle\langle\xi\rangle^{-\beta} .\right.
$$

Taking $\theta=2^{-1}$ in (4.3) we see that

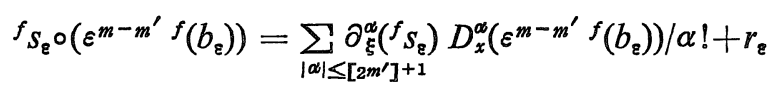

with $r_{\mathrm{\varepsilon}} \in S_{1,1 / 2}^{0}$ bounded in $\varepsilon$ and $a \in B$ in $S_{1,1 / 2}^{0}$.

From (4.4) each term in the summation can be written as $\varepsilon^{m-m^{\prime}}{ }^{f} a_{\varepsilon, \alpha}$ where $a_{\varepsilon, \alpha}=\sum_{\left(\alpha^{\prime}, \beta^{\prime}\right) \in D_{\alpha, 0}} g_{\alpha^{\prime}, \beta^{\prime}} \xi^{\beta^{\prime}} \partial_{x}^{\alpha^{\prime}} \partial_{\xi}^{\beta^{\prime}} b_{\varepsilon} \cdot \partial_{\xi}^{\alpha} S_{\varepsilon}$ with $g_{\alpha^{\prime}, \beta^{\prime}} \in C_{0}^{\infty}(\boldsymbol{R})$ depending only on $\alpha, \beta, \alpha^{\prime}$, 
$\beta^{\prime}, f$ so that $a_{\varepsilon, \alpha}$ is bounded in $S_{1,0}^{m-\alpha}$ in $\varepsilon$ and $a \in B$ in view of (4.2).

Now we have

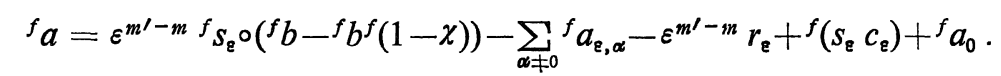

Since the case $m \leq 0$ is trivial from (4.3), one can prove (1) by induction on $[m]$ using (4.3) and applying induction hypothesis to the summation in (4.5). This completes the proof of (1).

(2) It is easy to check that

(4.6) there exists $C_{\alpha \beta \theta}$ for any $\theta \in(0,1)$ such that for any $e \in S_{1, \theta}^{k}$ with $k>0$,

$$
\left|\left(f^{k} e\right)_{(\alpha)}^{(\beta)}\right| \leq C_{\alpha \beta \theta}|a|_{|\alpha|+|\beta|}^{(k)}\langle f \xi\rangle^{k}\langle\xi\rangle^{\theta \alpha-\beta} .
$$

Suppose that $m>0$. Set $a_{0}=f^{m} a\langle f \xi\rangle^{-m}$. Then,

$$
a_{0} \circ\langle f \xi\rangle^{m}=\sum_{|\alpha| \leq[m /(1-\theta)]} \partial_{\xi}^{\alpha} a_{0} D_{x}^{\alpha}\left(\langle f \xi\rangle^{m}\right) / \alpha !+r, r \in S_{1, \theta}^{0} .
$$

Using (4.3) and Leibniz rule it is easy to see that for $\alpha \neq 0$

$$
a_{\alpha} \equiv i^{-\alpha}\left(a\langle f \xi\rangle^{-m}\right)^{(\alpha)}\left(\langle f \xi\rangle^{m}\right)_{(\alpha)} \in S_{1, \theta}^{m-(1-\theta) \alpha} .
$$

Now when $m>0$.

$$
f^{m} a=a_{0} \circ\langle f \xi\rangle^{m}-\sum_{0<|\alpha| \leq[m /(1-\theta)+1} \alpha !^{-1} f^{m} a_{\alpha}-r
$$

and when $m \leq 0$, (2) is trivial so that one can show (2) by induction on $[m /(1-\theta)]$. Indeed, when $m>0$, we only have to estimate the first and last term on the right of the above equality by using the boundedness of $a_{0}$ and $r$ on $L^{2}$ and (1) and apply the induction hypothesis to the summation by noting $[\{m-(1-\theta) \alpha\} /(1-\theta)]=[m /(1-\theta)]-\alpha$ and $\max (m-(1-\theta) \alpha, 0) \leq m$. The proof is complete.

The next lemma is one on the commutation.

Lemma 4.2. (1) Let $a_{i} \in S_{1,0}^{m}(\boldsymbol{R})$ with $s \geq 0$. Then there exists $C>0$ such that

(4.7) $\left\|\left[{ }^{f} a(x, D), f^{s}\right] u\right\| \leq C\left(\varepsilon\left\|\Lambda_{m} f^{s} u\right\|+\varepsilon^{-\max (m-1,0)}\|u\|\right), u \in \mathcal{S}, \varepsilon \in(0,1]$.

(2) Let $a_{i} \in S_{1,0}^{m_{i}}, i=1,2$. Then there exists $C>0$ such that with the motation $m=m_{1}+m_{2}$,

$$
\begin{gathered}
\left\|\left({ }^{f}\left(a_{1} a_{2}\right)-{ }^{f} a_{1} \circ{ }^{f} a_{2}\right)(x, D) u\right\| \leq C\left(\varepsilon\left\|\Lambda_{m} u\right\|+\varepsilon^{-\max (m-1,0)}\|u\|\right), \\
\text { for } u \in \mathcal{S}, \varepsilon \in(0,1] .
\end{gathered}
$$


Corollary 4.3. (1) Let $b \in S_{1,0}^{m}(\boldsymbol{R})$ and assume $b$ satisfies the assumption for $b$ in Lemma 4.1-(1). Then there exists $C>0$ such that

$$
C^{-1} \leq\left(\left\|f^{s} f b(x, D) u\right\|+\|u\|\right) /\left(\left\|\left.\right|^{f} b \circ f^{s}(x, D) u\right\|+\|u\|\right) \leq C, u \in \mathcal{S}, u \neq 0 .
$$

(2) Let $a_{i} \in S_{1,0}^{m_{i}}(\boldsymbol{R})$ and assume that $a_{i}$ satisfies the assumption for $b$ in Lemma 4.1-(1) for any $i$. Then there exists $C>0$ such that

$$
\begin{aligned}
& C^{-1} \leq\left(\|\|^{f}\left(a_{1} a_{2}\right)(x, D) u\|+\| u \|\right) /\left(\left\|\left.\right|^{f} a_{1} \circ^{f} a_{2}(x, D) u\right\|+\|u\|\right) \\
& \leq C, u \in \mathcal{S}, u \neq 0 \text {. }
\end{aligned}
$$

Corollary 4.3 follows from Lemma 4.2.

Proof of Lemma 4.2. (1) We prove (1) by induction on [m]. It is trivial if $m \leq 0$ and we assume $m>0$. From (3.64),

(4.11) for any positive $k, \varepsilon$, and $j \in \mathbb{N}$,

$$
f^{\varepsilon j}\left(\frac{d}{d x}\right)^{j}\left(f^{k}\right)=\psi_{\varepsilon, j, k} f^{k} \quad \text { for some } \quad \psi_{\varepsilon, j, k} \in C_{0}^{\infty}(\boldsymbol{R}) .
$$

From (4.3),

$$
f^{f} a f^{s}=\sum_{\alpha \leq[m]}^{f}\left(\partial_{\xi}^{\alpha} a\right) f^{\alpha} D_{x}^{\alpha}\left(f^{s}\right) / \alpha !+r \text { with } r \in S_{1,1 / 2}^{0} .
$$

From (4.11) and induction hypothesis, for $\alpha>0$,

$$
\left\|\left[{ }^{f}\left(\partial_{\xi}^{\alpha} a\right) f^{\alpha} D_{x}^{\alpha}\left(f^{s}\right)\right](x, D) u\right\| \leq C_{\alpha}\left(\left\|\Lambda_{m-1} f^{s} u\right\|+\varepsilon^{-\max (m-1,0)}\|u\|\right),
$$

the right hand side of which is estimated by that of (4.7) from Lemma 4.1(1). This proves (1).

(2) is proved in the similar way by using the fact that from (4.4), $f^{\alpha} D^{\alpha}\left({ }^{f} a_{2}\right)={ }^{f} a_{2, \alpha}$ for some $a_{2, \alpha} \in S_{1,0}^{m_{2}}$. The details are omitted.

Definition 4.4. Let $h \in \mathbb{N}, T \in(0,1)$ be given. Assume the notations in Definition 3.1 and set $\widetilde{\psi}_{1}=t \psi_{2}$. Let $d \in \mathbb{R}^{3}$ with $d_{1} \leq 0$. Then we say that $a(t, x, \xi) \in C^{\infty}\left(\boldsymbol{R}_{T}^{3}\right)$ belongs to $\Sigma^{d}=\Sigma^{d}(T, h)$ if for any $\alpha$

$$
\begin{gathered}
\left|\partial^{\alpha} a(t, x, \xi)\right| \leq C_{\alpha} \tilde{\psi}_{1^{1}-\alpha_{1}\left(\langle\xi\rangle^{-1 / 2}\right)^{d_{2}-\alpha_{2}} \psi_{3,1 / 2}^{d_{3}-\alpha_{3}}} \\
\text { with some constant } C_{\alpha} .
\end{gathered}
$$

We write $\Sigma=\Sigma(T, h)=\bigcup_{d}\left(\bigcup_{\rho} \Sigma_{\rho}^{d} \cup \Sigma^{d}\right)$.

Definition 4.5. We set for $\rho \in[0,1 / 2]$ and $d \in \mathbb{R}^{3}$

$$
\begin{aligned}
& \mu_{\rho}(d)=-\left(\rho d_{3}+2^{-1} d_{2}\right), \mu(d)=-2^{-1}\left(d_{1}+d_{3}\right), \nu(d)=-2^{-1} d_{2}+d_{3}, \\
& \sigma_{\rho}(d)=d_{3}+\max \left(0, \mu_{\rho}(d)-2^{-1} d_{1}\right), \sigma(d)=\nu(d)+\max (0, \mu(d)) .
\end{aligned}
$$


Then,

$$
\begin{aligned}
& \psi_{2}^{d_{2}} \psi_{3, \rho}^{d_{3}}=\left\langle t^{e}{ }_{h} \xi\right\rangle^{\mu_{\rho}(d)}\langle\xi\rangle^{d_{3}},
\end{aligned}
$$

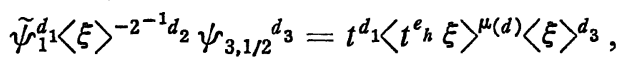

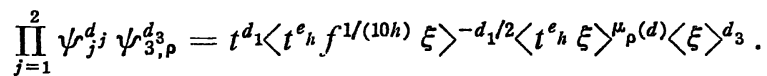

(4.14) $\left\{t^{-d_{1}} a_{1}(t, \cdot)\right\}_{t \in(0, T)}$ is bounded in $S_{1-\rho, 1 / 2}^{\sigma_{\rho}(d)}$ for $a \in \Sigma_{\rho}^{d}$, and in $S_{1 / 2,1 / 2}^{\sigma(d)}$ for $a \in \Sigma^{d}$.

A linear mapping on $\boldsymbol{R}^{3}$,

$$
A: d \rightarrow\left(d_{1}, \mu(d), \nu(d)\right)
$$

is one to one so that for any $d \in \boldsymbol{R}^{3}$ there exist $J_{\rho}(d), J(d), g_{\rho}(d), g(d) \in \boldsymbol{R}^{3}$ respectively determined uniquely such that

$$
\begin{aligned}
& A\left(J_{\rho}(d)\right)=\left(d_{1}, \mu_{\rho}(d)-d_{1} / 2, d_{3}\right), \\
& A(J(d))=(0, \mu(d), \nu(d)), \\
& A\left(g_{\rho}(d)\right)=\left(d_{1}, \max \left(0, \mu_{\rho}(d)-d_{1} / 2\right), \max \left(0, d_{3}\right)\right), \\
& A(g(d))=\left(d_{1}, \max (0, \mu(d)), \max (0, \nu(d))\right) .
\end{aligned}
$$

We set for $a \in C^{\infty}\left(\boldsymbol{R}_{T}^{3}\right)$ and $E \subset C^{\infty}\left(\boldsymbol{R}_{T}^{3}\right)$,

$$
{ }^{f} a(t, x, \xi)=a(t, x, f(x) \xi),{ }^{f} E=\left\{{ }^{f} a ; a \in E\right\} .
$$

Fact 4.6. 1. $a b \in \Sigma^{d+e}$ if $a \in \Sigma^{d}, b \in \Sigma^{e}$.

2. If $a \in \Sigma_{\rho}^{d}$ and $\varepsilon>0, f^{\varepsilon} \partial^{\alpha f} a={ }^{f} a_{\alpha, \varepsilon}$ with $a_{\alpha, \varepsilon} \in \Sigma_{\rho}^{d-\alpha}$.

3. If $a \in \Sigma^{d}$ and $\varepsilon>0, f^{\varepsilon} \partial^{\alpha f} a={ }^{f} a_{\alpha, \varepsilon}$ with $a_{\alpha, \varepsilon} \in \Sigma^{d-\alpha}$.

4. $\Sigma_{\rho}^{d} \subset \Sigma^{J_{\rho}^{(d)}}$.

5. $\left(\langle\xi\rangle^{-1 / 2}\right)^{J(d)_{2}} \cdot \psi_{3,1 / 2}^{J(d)_{3}}=\widetilde{\psi}_{1^{1}}^{d} \cdot\left(\langle\xi\rangle^{-1 / 2}\right)^{d_{2}} \cdot \psi_{3,1 / 2}^{d_{3}}$ and $\Sigma^{d}=t^{d_{1}} \Sigma^{J(d)}$.

6. $f \Sigma_{p}^{d} \subset \Sigma^{g_{p}}(d)$.

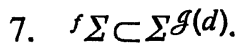

5 follows from (4.13) and 2,3,6,7 follow from the formula which follows from (4.4):

$$
\begin{aligned}
\partial^{\alpha}(f a(t, x, \xi))= & \sum_{\beta}\left(\delta^{2}-x^{2}\right)^{-2\left(\alpha_{2}-\beta_{2}\right)} \gamma_{\beta}(x) f^{\alpha}{ }^{\alpha}(x)(f(x) \xi)^{\beta_{3}-\sigma_{3}} \\
& \times^{f}\left(\partial^{\beta} a\right)(t, x, \xi),|x|<\delta,
\end{aligned}
$$

where the summation is taken over all the set $\left\{\beta=\left(\alpha_{1}, l, \alpha_{3}+j\right) ; l+k \leq \alpha_{2}, \alpha_{3}\right.$ $\left.+j \geq \min \left(\alpha_{2}-l, 1\right)\right\}$ and $\gamma_{\beta}$ is a polynomial depending only on $\alpha, \beta, f$ such that $\gamma_{\beta}=1$ for $\beta=\alpha$. 
We need Lemma 2 in [1] in this section and its easy extention in the next one. We recall the definition of a pair of weight functions in [1]. We say a pair of positive functions $\Phi, \varphi$ on $\boldsymbol{R}_{x}^{n} \times \mathbb{R}_{\xi}^{n}$ form a pair of weight functions if it satisfies the followings:

(4.17) there exist positive constants $C, c, \varepsilon$ such that (i) $c \leq \Phi(x, \xi) \leq$ $C(1+|\xi|), C \geq \varphi(x, \xi) \geq c(1+|\xi|)^{\varepsilon-1}$; (ii) $\varphi \Phi \geq c$; (iii) $\frac{\Phi(x, \xi)}{\varphi(x, \xi)} \sim \frac{\Phi(y, \eta)}{\varphi(y, \eta)}$ whenever $|\xi| \sim|\eta|$, where $A \sim B$ means that $|A| B \mid$ and $|B| A \mid$ are bounded; (iv) $C^{-1} \Phi(y, \eta) \leq \Phi(x, \xi) \leq C \Phi(y, \eta), C^{-1} \varphi(y, \eta) \leq \varphi(x, \xi) \leq$ $C \varphi(y, \eta)$ for all

$(y, \eta) \in U(x, \xi)=\{(y, \eta) ;|y-x|<c \varphi(x, \xi),|\eta-\xi|<c \Phi(x, \xi)\}$.

Then we have the following.

Lemma 4.7. Let $C>1, C_{0}>1, c>0, \varepsilon>0$, let $k, l \in N$, and let $\left(M_{1}, \cdots\right.$, $\left.M_{k+l}, m_{1}, \cdots, m_{k+l}\right)$ be a permutation of $2(k+l)$ real numbers. Let $B$ be a set of pairs of weight functions $(\Phi, \varphi)$ satisfying (4.17) with the $C, c$, $\varepsilon$ given above and that $\sup \left\{\frac{\Phi(x, \xi)}{\varphi(x, \xi)} \cdot \frac{\varphi(y, \eta)}{\Phi(y, \eta)} ;|\xi| \leq 4\right.$ and $|\eta| \leq 4$, or $\left.4^{-1}|\eta| \leq|\xi| \leq 4|\eta|\right\} \leq C_{0}$ according to (iii). Then, there exists constant $C^{\prime}>0$ and $L \in N$ having the following property: Suppose that $b(x, y, \xi, \eta),(x, y, \xi, \eta) \in \mathbb{R}^{2 n} \times \mathbb{R}^{2 n}$ is a smooth function in $(y, \eta)$ for each fixed $(x, \xi)$ and assume that for some $(\Phi, \varphi) \in B$,

$$
\sup _{x, y, \xi, \eta} D_{y}^{\alpha} D_{\eta}^{\beta} b \cdot\left(a_{\Phi, \varphi}^{|\alpha|,|\beta|}\right)^{-1}(x, y, \xi, \eta)<\infty \quad \text { for any } \alpha, \beta \in \mathbb{Z}_{+}^{n}
$$

where

$$
\begin{aligned}
a_{\Phi, \varphi}^{p, q}(x, y, \xi, \eta) & =\sum_{\substack{r_{1}+\cdots+r_{k}=q \\
r_{k+1}+\cdots+r_{k+l}=p, r_{1}, \cdots, r_{k+l} \in Z_{+}}} \prod_{j=1}^{k} \sup _{Q \in \mathbb{K}}\left(\Phi^{M_{j}-r_{j}}(Q) \varphi^{m_{j}}(Q)\right) \\
& \times \prod_{j=k+1}^{k+l} \sup _{Q \in \mathbb{K}}\left(\Phi^{M_{j}}(Q) \varphi^{m_{j}-r_{j}}(Q)\right)
\end{aligned}
$$

where $K$ is the covex hull of $(x, \xi),(x, \eta),(y, \xi),(y, \eta)$.

Then,

$$
a(x, \xi)=O S-\iint e^{-i y \cdot \eta} b(x, y+x, \xi, \eta+\xi) d y d \eta,
$$

where $O S-\iint e^{-i y \cdot \eta} d y d \eta$ denotes the oscillatory integral and the above one is well-defined by (4.17)-(i), satisfies the estimate

$$
\begin{aligned}
& |a(x, \xi)| \leq C^{\prime}|b|_{L}^{\Phi, \varphi}\left(\Phi^{\Sigma M_{j} \cdot \varphi^{\Sigma m} j}\right)(x, \xi), \\
& \text { where }|b|_{j}^{\Phi, \varphi}=\max _{|\alpha|+|\beta| \leq j} \sup _{x, y, \xi, \eta}\left|D_{y}^{\alpha} D_{\eta}^{\beta} b\right|\left(a_{\phi, \varphi}^{|\alpha|,|\beta|}\right)^{-1}(x, y, \xi, \eta) .
\end{aligned}
$$


Proof of Lemma 4.7 is parallel to that of Lemma 2 in [1], but we will give a brief discription of proof in the appendix.

Fact 4.8. Set $\Phi_{t}(x, \xi)=\psi_{1,1 / 2}(t, \xi), \varphi(x, \xi)=\langle\xi\rangle^{-1 / 2}$, for $(x, \xi) \in \boldsymbol{R} \times \boldsymbol{R}$. It is easy to check that for any $t \in(0, T),\left(\Phi_{t}, \varphi\right)$ is a pair of weight functions on $\boldsymbol{R}_{x} \times \boldsymbol{R}_{\xi}$ with $C=10, c=1 / 10, \varepsilon=1 / 2$, and that $\left\{\left(\Phi_{t}, \varphi\right) ; t \in(0, T)\right\}$ is a set of weight functions on $\boldsymbol{R}_{x} \times \boldsymbol{R}_{\xi}$ as $B$ in Lemma 4.7 .

Lemma 4.9. Let $a_{1} \in \Sigma^{d}, a_{2} \in \Sigma^{e}$ and set for $N \in \boldsymbol{N}$,

$$
r_{N}\left[a_{1}, a_{2}\right]=\int_{0}^{1} r_{\theta}\left[a_{1}, a_{2}\right](1-\theta)^{N-1} d \theta
$$

where

$$
r_{\theta}\left[a_{1}, a_{2}\right]=(2 \pi)^{-1} \text { OS }-\iint e^{-i y \cdot \eta} a_{1}(t, x, \xi+\theta \eta) a_{2}(t, x+y, \xi) d y d \eta .
$$

Then, $r_{N}\left[a_{1}, a_{2}\right] \in \Sigma^{d+e}$.

Lemma 4.10. Let $a \in \Sigma^{d}$ and $g(\theta, x, y) \in C^{\infty}\left([0,1] \times \boldsymbol{R}^{2}\right)$ with $\sup _{\theta, x, y}\left|\partial_{x, y}^{\alpha} g\right|$ $<\infty$ for all $\alpha$. We set

$$
r_{\theta}(t, x, \xi)=O S-\iint e^{-i y \cdot \eta} g(\theta, x, y) a(t, x+\theta y, \xi+\theta \eta) d y d \eta
$$

Then

$$
\sup _{\theta, t, x, \xi}\left|\partial_{t, x, \xi}^{\alpha} r_{\theta}\right| \tilde{\psi}_{1}^{-d_{1}+\alpha_{1}}\left(\langle\xi\rangle^{-1 / 2}\right)^{-d_{2}+\alpha_{2}} \psi_{1,1 / 2}^{-d_{3}+\alpha_{3}}<\infty \text { for any } \alpha .
$$

Remark 4.11. Let $a_{\theta}(t, x, y, \xi, \eta) \in C^{\infty}\left([0,1]_{\theta} \times \boldsymbol{R}_{x, y, \xi, \eta}^{4 n}\right)$ satisfying that with $\delta \in[0,1)$ and $M \geq 0$

$$
\left|\partial_{t}^{i} \partial_{x}^{\alpha} \partial_{y}^{\beta} \partial_{\xi}^{\gamma} \partial_{\eta}^{\nu} a_{\theta}(t, x, y, \xi, \eta)\right| \leq C_{i \alpha \beta \gamma \nu}(t, x, \xi)(1+|y|)^{M}(1+|\eta|)^{M+\delta|\beta|}
$$

where $C_{i \alpha \beta \gamma \nu}$ is assumed to be locally bounded in $(t, x, \xi)$ and to be independent of $\theta$. Then

(1) for any compact $K \subset \boldsymbol{R}^{2 n}, O S-\iint e^{-i y \cdot \eta} a_{\theta}(t, x, y, \xi, \eta) d y d \eta$ is continuous in $(\theta, x, \xi) \in[0,1] \times K$,

(2) one can differentiate the above oscillatory integral in $(t, x, \xi)$ by differentiation under integral sign any times. These are easily verified from the definition of the oscillatory integral.

Proof of Lemma 4.9. We assume the notations in Fact 4.8. From Remark 4.11

$$
\partial^{\alpha} r_{N}\left[a_{1}, a_{2}\right]=\sum_{\beta \leq \alpha}\left(\begin{array}{c}
\alpha \\
\beta
\end{array}\right) r_{N}\left[\partial^{\alpha-\beta} a_{1}, \partial^{\beta} a_{2}\right]
$$


Setting $b_{\beta \theta}(t, x, y, \xi, \eta)=\left(\partial^{\alpha-\beta} a_{1}\right)(t, x, \xi+\theta \eta)\left(\partial^{\beta} a_{2}\right)(t, x+y, \xi)$, we have in view of Fact 4.6-5 that there exists $C_{i j}$ such that for any $\beta \leq \alpha$,

$$
\begin{aligned}
\left|\partial_{y}^{i} \partial_{\eta}^{j}\left(b_{\beta \theta}(t, x, y-x, \xi, \eta-\xi)\right)\right| \leq & C_{i j} t^{d_{1}+e_{1}-\alpha_{1}} \sup _{Q \in \xi_{\eta}} \Phi_{t}^{m_{1}} \cdot \varphi^{m_{2}}(Q) \\
& \times \sup _{Q \in \xi_{\xi \eta}} \Phi_{t}^{m_{1}^{\prime}} \cdot \varphi^{m_{2}^{\prime}}(Q),
\end{aligned}
$$

where $\overline{\xi \eta}$ is the segment joining $\xi$ to $\eta$ and

$$
m_{1}=J(d-\alpha+\beta)_{3}-j, m_{2}=J(d-\alpha+\beta)_{2}, m_{1}^{\prime}=J(e-\beta)_{3}, m_{2}^{\prime}=J(e-\beta)_{2}-i .
$$

By virtue of Fact 4.8 we get from (4.23) and Lemma 4.7 that there exists $C_{a}$ such that for any $\beta \leq \alpha$,

$$
\begin{aligned}
& \left|r_{\theta}\left[\partial^{\alpha-\beta} a_{1}, \partial^{\beta} a_{2}\right]\right| \leq C_{\alpha} t^{d_{1}+e_{1}-\alpha_{1}}\left\{\Phi_{t}^{J(d+e-\alpha)_{3}} \cdot \varphi^{J\left(d+e-\alpha_{2}\right)}\right\}(\xi) \\
& \quad \text { for } \theta \in[0,1] .
\end{aligned}
$$

(4.22) and (4.24) prove Lemma 4.9.

Lemma 4.10 is proved in the similar way. The proof is omitted.

Corollary 4.12. In the following, for (ii) and (iv) we suppose that $a \in \Sigma^{d}$, $b \in \Sigma^{e}$ with $\nu(d) \geq 0, \mu(d) \geq 0$, and for the others we supose that $a \in \Sigma_{\rho}^{d}, b \in \Sigma^{e}$ with $d_{3} \geq 0, \mu_{\rho}(d) \geq 0$.

Then we have

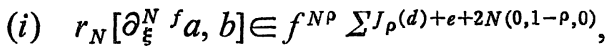

(ii) $r_{N}\left[\partial_{\xi}^{N} f a, b\right] \in f^{N / 2} \Sigma^{d+e+N(0,1,0)}$,

(iii) $r_{N}\left[\partial_{\xi}^{N} f a, D_{x}^{N} b\right] \in f^{N \rho} \Sigma^{J_{\rho}(d)+e+N(0,1-2 \rho, 0)}$,

(iv) $r_{N}\left[\partial_{\xi}^{N} f a, D_{x}^{N} b\right] \in f^{N / 2} \sum^{d+e}$,

(v) $\quad r_{M}^{*}\left[\partial_{\xi}^{N} D_{x}^{N} f a\right] \in f^{N \rho / 2} \Sigma^{J_{\rho}(d)+N(0,1-2 \rho, 0)}+t^{d_{1}} \Sigma^{(0, N, 0)}$

where by definition,

$$
r_{M}^{*}[c]=\int_{0}^{1} r_{\theta}^{\sharp}[c](1-\theta)^{M-1} d \theta
$$

where

$$
r_{\theta}^{\sharp}[c]=(2 \pi)^{-1} O S-\iint e^{-i y^{\eta}} c(t, x+\theta y, \xi+\theta \eta) d y d \eta .
$$

Proof. (i) $f \sum_{\rho}^{d} \subset \Sigma^{J_{\rho}^{(d)}}$ from Fact 4.6-4 and 7, the assumption on $d$, and the definition $\mathcal{G}, J_{\rho}$, and we have

$$
\begin{aligned}
& \partial_{\xi}^{N} f a=f^{N \rho} a_{N} \text { with } \\
& a_{N}=(f\langle\xi\rangle\langle f \xi\rangle)^{(1-\rho) N}\langle\xi\rangle^{-(1-\rho) N}\langle f \xi\rangle^{(1-\rho) N} f \partial_{\xi}^{N} a .
\end{aligned}
$$

Using (4.3) and (4.6), and noting $\langle\xi\rangle^{(1-\rho) N} \partial_{\xi}^{N} a \in \Sigma^{J_{\rho}(d)}$ we see that $a_{N} \in$ 


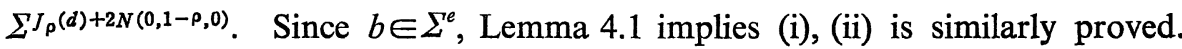
(iii), (iv) are particular cases of (i), (ii) respectively. Next we consider (v). Using (4.26) and Leibniz rule we see that $D_{\xi}^{N} \partial_{x}^{N}{ }^{f} a=f^{2 N \mathrm{P} / 3} \tilde{a}_{N}$ for some $\tilde{a}_{N} \in$ $\Sigma^{J_{\rho}(d)+N(0,1-2 \rho, 0)}$ so that

$$
\begin{aligned}
& r_{M}^{*}\left[\partial_{\xi}^{N} D_{x}^{N} f a\right] \\
& =(2 \pi)^{-1} \int_{0}^{1}(1-\theta)^{M-1}\left(O S-\iint e^{-i y \eta} f^{2 N \rho / 3}(x+\theta y)\right. \\
& \left.\quad \cdot \tilde{a}_{N}(t, x+\theta y, \xi+\theta \eta) d y d \eta\right) d \theta
\end{aligned}
$$

Using Taylor expansion at $x$ of order $N_{1}$ and integrating by parts in $\eta$ we see that the right hand side of (4.27) equals to, with some $g(\theta, x, y) \in$ $C^{\infty}\left([0,1] \times \boldsymbol{R}^{2}\right)$ satisfying $\sup _{\theta, x, y}\left|\partial_{x, y}^{\alpha} g\right| \leq C_{N, N_{1}, \rho}$ for any $\alpha$,

$$
\begin{aligned}
& (2 \pi)^{-1}\left\{\sum_{j<N N_{1}} j !^{-1}\left(\frac{d}{d x}\right)^{j}\left[f^{2 N \rho / 3}(x)\right]\right. \\
& \quad \times \int_{0}^{1} \theta^{2 j}(1-\theta)^{M-1}\left(O S-\iint e^{-i y^{\eta}} D_{\xi}^{j} \tilde{a}_{N}(t, x+\theta y, \xi+\theta \eta) d y d \eta\right) d \theta \\
& \left.\quad+\int_{0}^{1} \theta^{2 N_{1}}(1-\theta)^{M-1}\left(O S-\iint e^{-i y^{\eta}} D_{\xi}^{N} \tilde{a}_{N}(t, x+\theta y, \xi+\theta \eta) g(\theta, x, y) d y d \eta\right) d \theta\right\} .
\end{aligned}
$$

By Lemma 4.10 the integral in each term of the summation belong to $\Sigma^{J_{\rho}(d)+N(0,1-2 \rho, 0)}$ and the last term does to $\Sigma^{\tilde{d}}$ with $\tilde{d}=J_{\rho}(d)+N(0,1-2 \rho, 0)$ $-\left(0,0, N_{1}\right)$. Since $N_{1}$ is an arbitrary positive integer, we have proved $(v)$ in view of (3.64) and Lemma 3.8-5. The proof is complete.

We define $a_{t} \in C^{\infty}\left((0, T) ; C^{\infty}\left(\boldsymbol{R}^{2}\right)\right)$ for $a \in C^{\infty}\left(\boldsymbol{R}_{T}^{3}\right)$ by $a_{t}(\cdot)=a(t, \cdot)$. Then taking account of (4.14),

$$
\begin{aligned}
& a_{t} \circ b_{t}=\sum_{\alpha<N} \partial_{\xi}^{\alpha} a_{t} \cdot D_{x}^{\alpha} b_{t} / \alpha !+r_{N}\left[\partial_{\xi}^{N} a, D_{x}^{N} b\right] /(N-1) !, \\
& a_{t}^{\sharp}=\sum_{\alpha<N} \partial_{\xi}^{\alpha} D_{x}^{\alpha} \bar{a}_{t} / \alpha !+r_{N}^{*}\left[\partial^{N} D_{x}^{N} \bar{a}\right] /(N-1) !,
\end{aligned}
$$

for $a, b \in \Sigma$ where in general we denote by $c^{*}$ the symbol in $S_{\rho, \theta}^{m}$ of formally adjoint of the operator $c(x, D), c \in S_{\rho, \theta}^{m}(\boldsymbol{R})$ on $\mathcal{S}(\boldsymbol{R})$.

We also define $a \circ b \in \Sigma, a^{\sharp} \in C^{\infty}\left(\boldsymbol{R}_{T}^{3}\right)$ for $a, b \in \Sigma$ by

$$
(a \circ b)_{t}=a_{t} \circ b_{t}, \quad a_{t}^{*}=a_{t}^{\sharp} .
$$

Lemma 4.13. $a \circ b \in \Sigma^{d+e}$ if $a \in \Sigma^{d}, b \in \Sigma^{e}$.

This is an immediate consequence of (4.28), Lemma 4.9, and Fact 4.6-1.

Lemma 4.14. (1) Let $a \in \Sigma_{\rho}^{d}$ and suppose that $\mu(d) \geq 0$. Then there exists 
C $>0$ such that

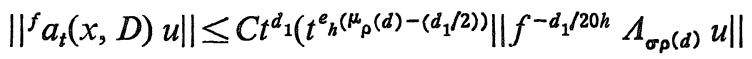

$$
\begin{aligned}
& \left.+t^{e{ }{ }{ }_{\rho}(d)}\left\|\Lambda_{v_{\rho}(d)+d_{3}} u\right\|+\left\|\Lambda_{d_{3}} u\right\|+\|u\|\right) \quad \text { for } u \in \mathcal{S}, t \in(0, T) \text {. }
\end{aligned}
$$

(2) Let $a \in \Sigma^{d}$ and suppose that $\mu(d) \geq 0$. Then there exists $C>0$ such that

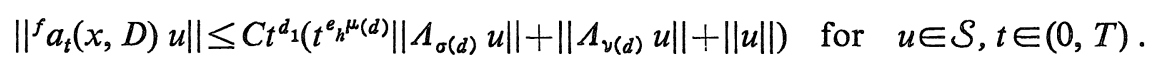

(3) Let $a \in \Sigma^{d}$ and suppose that $\mu(d) \geq 0$, then there exists $C>0$ such that

$$
\begin{aligned}
& \left\|a_{t}(x, D) u\right\| \leq C t^{d_{1}}\left(t^{e} h^{\mu(d)}\|u\|_{\sigma(d)}+\|u\|_{\nu(d)}+\|u\|\right) \text { for } u \in \mathcal{S}, t \in(0, T) \\
& \quad \text { where }\|u\|_{s}=\left\|\langle D\rangle^{s} u\right\| .
\end{aligned}
$$

(4) Let $a \in \Sigma^{d}$ and suppose that $\mu(d) \geq 0$. Then there exists a constant $C>0$ such that

$$
\begin{aligned}
& \left.\left\|f^{\max (\sigma(d), 0)} a_{t}(x, D) u\right\| \leq C t^{d_{1}\left(t^{e_{h}}{ }^{\mu(d)}\right)}\left\|\Lambda_{\sigma(d)} u\right\|+\left\|\Lambda_{\nu(d)} u\right\|+\|u\|\right) \\
& \quad \text { for } u \in \mathcal{S}, t \in(0, T) .
\end{aligned}
$$

Fact 4.15. Set $\left.\Psi_{1}=t^{s}\langle\xi\rangle, \Psi=f^{s^{\prime}} t^{s}\langle\xi\rangle, t \in(0,1), \xi \in \boldsymbol{R},|x|\langle\delta ; s\rangle 0, s^{\prime}\right\rangle$ 0 . Then for any $\varepsilon>0$,

$$
\begin{aligned}
& \left|\partial^{\alpha} \Psi_{1}\right| \leq C_{\alpha} \Psi_{1} t^{-\alpha_{1}\langle\xi\rangle^{1-\alpha_{3}}} \\
& \left|\partial^{\alpha} \Psi_{2}\right| \leq C_{\alpha \varepsilon} \Psi_{2}\left(\left\langle t^{s} \xi\right\rangle \Psi_{2}^{-1}\right)^{2 \alpha_{2}} t^{-\alpha_{1}}\langle\xi\rangle^{-\alpha_{3}} .
\end{aligned}
$$

Proof of Lemma 4.14. (1) Set

$$
\Psi=t^{d_{1}}\left(t^{e_{h} \mu_{\rho}(d)}\langle\xi\rangle^{\mu_{\rho}(d)}+1\right)\langle\xi\rangle^{d_{3}}\left(t^{-e_{h} d_{1} / 2} f^{-d_{1} /(20 h)}\langle\xi\rangle^{-d_{1} / 2}+1\right) .
$$

Then it is easy to see using Fact 4.15 and Lemma 3.8-3 that

$$
\text { for any } \varepsilon>0, \alpha \in \mathbb{Z}_{+}^{3},\left|\partial^{\alpha} \Psi\right| \leq C_{\alpha \varepsilon} t^{-\alpha_{1}\left\langle t^{e} h \xi\right\rangle^{\alpha \alpha}{ }_{2}\langle\xi\rangle^{-\alpha_{3}} \Psi} \text {. }
$$

Using (4.32) and (4.16) it is easy to check that $\partial^{\alpha} \Psi \in t^{-\alpha_{1}} \Sigma_{\rho}^{d^{\alpha}}$ with $d^{\alpha}=d+\alpha_{3}$ $(0,2 \rho,-1)+\varepsilon \alpha_{2}(0,-2,0)$ for any $\varepsilon>0$ and $\alpha \in \mathbb{Z}_{+}^{3}$, and that the estimate $\left|\partial^{\alpha} f \Psi\right| \leq C_{\alpha \varepsilon} t^{-\alpha_{1}}\langle\xi\rangle^{\varepsilon \alpha_{2}-\alpha_{3} f} \Psi$ holds for any $\varepsilon>0$ and $\alpha \in \mathbb{Z}_{+}^{3}$. In particular,

$$
\begin{aligned}
& f^{\varepsilon} \partial_{x}^{k} f \Psi \in{ }^{f} \sum_{\rho}^{d+k / 4(0,-2,0)}, \partial_{x}^{k} f \Psi \in \Sigma^{J_{\rho}(d)+\varepsilon(0,-1,0)}, \\
& \quad \text { for any } \varepsilon>0 .
\end{aligned}
$$

Setting $b=a \Psi^{-1}$, and using (4.28), (4.32), (4.33), and Corollary 4.12-(i) we see that

$$
\begin{aligned}
& b \in \Sigma_{\rho}^{0}, \Psi \in \Sigma_{\rho}^{d},{ }^{f} b \circ f \Psi={ }^{f} a+{ }^{f} a_{1}+{ }^{f} a_{2} \text { for some } \\
& a_{1} \in \Sigma^{d^{\prime}}, a_{2} \in t^{d_{1}} \Sigma^{0} \quad \text { with } d^{\prime} \text { satisfying }
\end{aligned}
$$




$$
\sigma_{\rho}\left(d^{\prime}\right) \leq \sigma_{\rho}(d)-1 / 4, \mu_{\rho}\left(d^{\prime}\right)=\mu_{\rho}(d), d_{1}^{\prime}=d_{1} .
$$

If we denote by $S_{d}(u)$ the expression defined by deleting $C$ from the right hand side of the inequality in (1), we have that

$$
\begin{gathered}
S_{d^{\prime}}(u) \leq C S_{d}(u) \text { and } \quad\left\|f \Psi_{t}(x, D) u\right\| \leq C S_{d}(u), \\
\text { with } C \text { independent of } t \in(0,1], u \in \mathcal{S} .
\end{gathered}
$$

Indeed, the latter is clear and the former is easily shown by the properties of $d^{\prime}$, Corollary 4.3-(1), and Lemma 4.1-(1).

Now, since (1) is clear for $d$ with $\sigma_{\rho}(d) \leq 0$, in particular with $\left[4 \sigma_{\rho}(d)\right] \leq$ $-1,(1)$ can be shown by induction in $\left[4 \sigma_{\rho}(d)\right]$ in view of $(4.34),(4.35),(4.14)$, and Fact 4.6.

(2) and (3). They can be proved similarly by induction on $[2 \sigma(d)]$ by setting $\Psi=t^{d_{1}}\left(t^{e_{h} h^{\mu(d)}}\langle\xi\rangle^{\mu(d)}+1\right)\langle\xi\rangle^{\nu(d)}$ instead of the $\Psi$ in the proof of (1) and by using for (3) the standard interpolation in the Sobolev spaces instead of Lemma 4.1-(1). We omit the proofs.

(4) is clear when $\left[2 \sigma\left(d^{\prime}\right)\right] \leq-1$, and we prove it by induction on $[2 \sigma(d)]$. Assume $\sigma(d) \geq 0$ and (4) is true for $d^{\prime}$ with $\left[2 \sigma\left(d^{\prime}\right)\right] \leq[2 \sigma(d)]-1$. It is easy to see that

$$
\begin{gathered}
{\left[a_{t}(x, D), f^{\sigma(d)}\right]=f^{\max (\sigma(d-(0,0,1)), 0)} a_{1 t}(x, D)+a_{2}(x, D)} \\
\quad \text { for some } a_{1} \in \Sigma^{d-(0,0,1)}, a_{2} \in t^{d_{1}} \Sigma^{0} .
\end{gathered}
$$

Let $S_{d}(u)$ be the expression defined by removing the right of the inequality of (4). Then,

$$
\begin{aligned}
& S_{d-(0,0,1)}(u) \leq C S_{d}(u) \text { and }\left\|a_{t}(x, D) f^{\sigma(d)} u\right\| \leq C S_{d}(u) \text { with } C \\
& \text { independent of } t \in(0,1], u \in \mathcal{S} .
\end{aligned}
$$

In fact, the former follows from Lemma 4.1-(1) and the latter does from Lemma 4.1-(1) and the inequality

$$
\text { If } s^{\prime} \geq s>0,\left\|f^{s^{\prime}} u\right\|_{s} \leq C_{s s^{\prime}}\left(\left\|\Lambda_{s} u\right\|+\|u\|\right), u \in \mathcal{S},
$$

which can be shown by expanding the symbol $\langle\xi\rangle^{s} \circ f^{s^{\prime}}$ and using Lemma 4.1-(2), -(1) in this order. (4.36), (4.37), and the induction hypothesis show (4). The proof is complete.

Remark on notations. The letters $T$ and $h$ in the definitions of $\Sigma, B_{h, T}$ are in common for all notations if we do not write these letters.

Recall the definition of $\mathcal{S}_{T}((2.11))$. If $a \in \Sigma(T, h)$ and $u \in \mathcal{S}_{T}, a_{t}(x, D) u_{t}$ 
as a function of $(t, x) \in(0, T) \times \boldsymbol{R}$ also belongs to $\mathcal{S}_{T}$ and $\partial_{t}\left(a_{t}(x, D) u_{t}\right)=$ $\left(\partial_{t} a\right)_{t}(x, D) u_{t}+a_{t}(x, D)\left(\partial_{t} u\right)_{t}$.

Definition 4.16. For $a \in \Sigma(T, h)$ and, we define the linear operator on $\mathcal{S}_{T}$, which we denote by $O p a$ or $a(t, x, D)$, by assigning $u$ to the function $(t, x) \rightarrow$ $\left(a_{t}(x, D) u_{t}\right)(x)$. Furthermore, let $\mathscr{P}_{T}$ be the set of all $q(t, x, \tau, \xi) \in C^{\infty}\left(\boldsymbol{R}_{T}^{4}\right)$ being a polynomial in $\tau$ with coefficients in $\Sigma\left(T_{0}, h\right)$ in $(t, x, \xi)$. With the unique expression $q(t, x, \tau, \xi)=\sum_{k \leq m} a_{k}(t, x, \xi) \tau^{k}$ we define the operator on $\mathcal{S}_{T}$, denoted by $O p q$ or $q(t, x, D)$, sending $u$ to $\sum_{k \leq m} a_{k}(t, x, D) D_{t}^{k} u$.

$q$ is determined by $O p q$ in the above. Indeed, $(O p q) u=0$ for any $u$ means $q=0$, since for $u=\chi\left(t ; t_{0}\right)\left(t-t_{0}\right)^{k} v(x)$ where $k \leq m, \chi \in C_{0}^{\infty}((0, T))$ with $\chi=1$ near $t_{0} \in(0, T)$, and $v \in \mathcal{S}$ and $q$ as above, $u \in \mathcal{S}_{T}$ and $(O p q) u\left(t_{0}, \cdot\right)=$ $i^{-k} k ! a_{k}\left(t_{0}, x, D\right) v$. We call $q$ the symbol of $O p q$.

For $q_{j} \in \mathscr{P}_{T}, j=1,2$ with $q_{j}=\sum_{k \leq m_{j}} a_{j k} \tau^{k}, a_{j k} \in \Sigma$, we define $q_{1} \circ q_{2}$ by

$$
\begin{aligned}
& q_{1} \circ q_{2}(t, x, \tau, \xi) \\
& \quad=O S-\iint e^{-i y \eta} \sum_{j \leq m_{1}}(2 \pi j !)^{-1} \partial_{\tau}^{j} q_{1}(t, x, \tau, \xi+\eta) D_{t}^{j} q_{2}(t, x+y, \xi) d y d \eta .
\end{aligned}
$$

Then,

$$
\begin{aligned}
& q_{1} \circ q_{2}(t, x, D)=q_{1}(t, x, D) q_{2}(t, x, D) \\
& q_{1} \circ q_{2}=\sum_{1 \leq m_{1}} l !^{-1}\left(\sum_{s=0}^{N-1} s !^{-1} \partial_{\xi}^{s} \partial_{\tau}^{l} q_{1} \circ D_{x}^{s} D_{t}^{l} q_{2}\right. \\
& \quad+\left(N_{l}-1\right) !^{-1} r_{N_{l}}\left[\partial^{N} \partial_{\tau}^{l} q_{1}, D_{x}^{N} D_{t}^{l} q_{2}\right] \text { for any } N_{l} \in \mathbb{N}
\end{aligned}
$$

where, by definition,

$$
r_{N}\left[q_{1}, q_{2}\right]=\sum_{\substack{k \leq m_{1} \\ j \leq m_{2}}} r_{N}\left[a_{1 k}, a_{2 j}\right] \tau^{k+j} .
$$

Actually these are true when $q_{i}$ are monomials in $\tau$ so that they are true in general, since all terms are bilinear in $q_{1}$ and $q_{2}$.

For $E \subset \Sigma(T, h)$, we set

$O p E=$ the set of all operators with the symbol in $E$.

Definition 4.17. For real numbers $k \geq 0, l \geq 0, N \geq k$ we define $A^{k, l, N}$ (resp. $\left.B^{k, l, N}\right)=$ the set of all symbols being a finite sums of symbols of the form:

$$
\begin{aligned}
& a+f^{N} b ; a \in{ }^{f} \sum_{1 / 2}^{d}\left(\text { resp. }{ }^{f} \Sigma^{d}\right), b \in \Sigma^{e} \text { with } \\
& \left.d \in \Delta^{k, l}, e \in \Delta_{k, l} \text { (resp. } d, e \in \Delta_{k, l}\right)
\end{aligned}
$$

where 


$$
\begin{aligned}
& \Delta^{k, l}=\left\{d \in \boldsymbol{R}^{3} ; 0 \geq d_{1} \geq-2 l, \mu_{1 / 2}(d) \geq 0, d_{3} \geq 0, \sigma_{1 / 2}(d)=k\right\}, \\
& \Delta_{k, l}=\left\{d \in \boldsymbol{R}^{3} ; 0 \geq d_{1} \geq-2 l, \tilde{\mu}(d) \geq 0, \nu(d) \geq 0, \sigma(d)=k\right\}
\end{aligned}
$$

with the notation

$$
\tilde{\mu}(d)=\mu\left(\left(0, d_{2}, d_{3}\right)\right),
$$

and we set

$$
\begin{aligned}
& \mathcal{A}^{k, l, N}\left(\operatorname{resp} . \mathscr{B}^{k, l, N}\right)=O p A^{k, l, N}\left(\operatorname{resp} . O p B^{k, l, N}\right), \\
& A^{k, N}\left(\operatorname{resp} . B^{k, N}\right)=A^{k, 0, N}\left(\operatorname{resp} . B^{k, 0, N}\right) \\
& \mathscr{A}^{k, N}\left(\operatorname{resp} . \mathscr{B}^{k, N}\right)=\mathscr{A}^{k, 0, N}\left(\operatorname{resp} . \mathscr{B}^{k, 0, N}\right)
\end{aligned}
$$

From Fact 4.6-4

$$
A^{k, l, N} \subset B^{k, l, N}
$$

and clearly,

$$
\begin{aligned}
& A^{k_{1}, l_{1}, N_{1}}\left(\text { resp. } B^{k_{1}, l_{1}, N_{1}}\right) \subset A^{k_{2}, l_{2}, N_{2}}\left(\text { resp. } B^{k_{2}, l_{2}, N_{2}}\right) \\
& \text { if } k_{1} \leq k_{2}, l_{1} \leq l_{2}, N_{1} \geq N_{2} .
\end{aligned}
$$

In general, for a ring $R$ and $E_{i} \subset R, a_{i} \in R, i \in$ finite set $I$, the set $\sum_{i \in I} a_{i} E_{i}$ is defined by $\left\{\sum_{i \in I} a_{i} e_{i} ; e_{i} \in E_{i}\right\}$. We use this notation for the ring of symbols with the product as functions and the one of their defining operators on $\mathcal{S}_{T}$ in Definition 4.16.

\section{Lemma 4.18.}

(1) $A_{1} A_{2} \in \mathcal{A}^{k_{1}+k_{2}, l_{1}+l_{2}, N}$ (resp. $\mathscr{B}^{k_{1}+k_{2}, l_{1}+l_{2}, N}$ )

if $A_{i} \in \mathcal{A}^{k_{i}, l_{i}, N}$ (resp. $\left.\mathscr{B}^{k_{i}, l_{i}, N}\right)$ and $N \geq k_{1}+k_{2}$.

(2) If $b \in C^{\infty}\left(\boldsymbol{R}_{T}^{3}\right)$ satisfies with $d \in \Delta_{k_{1}, 0}$,

$$
\begin{gathered}
\left|\partial^{\alpha} b(t, x, \xi)\right| \leq C_{\alpha} t^{-\alpha_{1}}\left(\langle\xi\rangle^{-1 / 2}\right)^{d_{2}} \psi_{3,1 / 2}{ }^{d_{3}}\langle\xi\rangle^{-\alpha_{3}} \text { for all } \alpha, \\
O p\left(a \circ^{f} b-a^{f} b\right), O p\left({ }^{f} b \circ a-a^{f} b\right) \in \mathscr{B}^{\max \left(k_{1}+k_{2}-1 / 2,0\right), N} \\
\text { for } a \in B^{k_{2}, N}, N \geq \max \left(k_{1}+k_{2}, 1\right) .
\end{gathered}
$$

(3) Suppose that $a \in \Sigma_{1 / 2}^{(0,-1,1)}$ satisfies

$$
\partial_{x} a \in \Sigma_{1 / 2}^{(0,-1,1)}, \partial_{\xi} a \in \Sigma_{1 / 2}^{0} .
$$

Then we have the followings:

(i) $O p\left({ }^{f} a \circ b-{ }^{f} a \cdot b\right), O p\left(b \circ{ }^{f} a-b \cdot{ }^{f} a\right) \in \mathcal{A}^{k+1 / 2, l, N}\left(\right.$ resp. $\left.\mathscr{B}^{k+1 / 2, l, N}\right)$ if $b \in A^{k, l, N}$ (resp. $B^{k, l, N}$ ) with $N \geq 1 / 2+k$. 
(ii) $O p{ }^{f} \bar{a}-\left(O p{ }^{f} a\right)^{*} \in \mathcal{A}^{1 / 2, N}+O p \Sigma^{(0, N, 0)}$ for any $t$ and $N \geq 1$, where for $q=\sum_{k} a_{k} \tau^{k} \in \mathscr{Q}_{T}$ we denote by $(O P q)^{*}$ the operator with the symbol $q^{*}=$ $\sum_{k} \sum_{j=0}^{k}\left(\begin{array}{l}k \\ j\end{array}\right) D_{t}^{k-j} a_{k}^{k} \tau^{j}$ so that

$$
((O p q) u, v)=\left(u,(O p q)^{*} v\right) \text { for } u, v \in \mathcal{S}_{T},
$$

where $(, \quad)$ denotes the inner product if $L^{2}((0, T) \times \boldsymbol{R})$.

(iii) If $b$ satisfies (4.41), $\left[O p^{f} a, O p^{f} b\right] \in O p{ }^{f} \Sigma^{d}+f^{N} O p \Sigma^{(0, N, 0)}$ for any $N \geq 1$.

(iv) If $b$ satisfies (4.42), $O p\left({ }^{f} a \circ^{f} b-{ }^{f}(a b)\right) \in \mathcal{A}^{1, N}$ for any $N \geq 1$.

(4) For any $a \in \Sigma_{\rho}^{d}\left(\right.$ resp. $\left.\Sigma^{d}\right), s \in[0, \infty)$, and $N \in N$, there exists $b \in$ $\Sigma_{\rho}^{d-(0,0,1)}\left(\right.$ resp. $\left.\Sigma^{d-(0,0,1)}\right)$ such that

$$
\operatorname{supp} b \subset \operatorname{supp} a, f^{s} \circ f^{f} a-\left(f^{f} a+{ }^{f} b\right) \circ f^{s} \in f^{N} t^{d_{1}} \Sigma^{(0, N, 0)} .
$$

(4)' For any $a \in \Sigma_{\rho}^{d}\left(\right.$ resp. $\left.\Sigma^{d}\right), s \in[0, \infty)$, and $N \in N$, there exists $b \in \Sigma_{\rho}^{d-(0,0,1)}$ (resp. $\left.\Sigma^{d-(0,0,1)}\right)$ such that

$$
\operatorname{supp} b \subset \operatorname{supp} a,{ }^{f} a \circ f^{s}-f^{s}\left({ }^{f} a+{ }^{f} b\right) \in f^{N} t^{d_{1}} \Sigma^{(0, N, 0)} .
$$

(5) For any $a \in \Sigma^{d}, s \in(0, \infty)$, and $\varepsilon \in(0, s)$, and $N \in \mathbb{N}$ there exists $b \in$ $\Sigma^{d-(0,0,1)}$ such that

$$
f^{s} a-a \circ f^{s}-b \circ f^{s-\varepsilon} \in t^{d_{1}} \Sigma^{(0, N, 0)} .
$$

Proof. (1) Let us prove the former case. We may assume

$$
A_{i}=O p\left({ }^{f} a_{i}+f^{N} b_{i}\right), \text { with } a_{i} \in \Sigma_{1 / 2}^{d^{i}}, b_{i} \in \Sigma^{e^{i}}, d^{i} \in \Delta^{k_{i}, l_{i}}, e^{i} \in \Delta_{k_{i}, l_{i}} .
$$

Now,

$$
\left({ }^{f} a_{1}+f^{N} b_{1}\right) \circ\left({ }^{f} a_{2}+f^{N} b_{2}\right)={ }^{f} a_{1} \circ{ }^{f} a_{2}+{ }^{f} a_{1} \circ\left(f^{N} b_{2}\right)+f^{N} b_{1} \circ\left({ }^{f} a_{2}+f^{N} b_{2}\right),
$$

and the first term $\in A^{k_{1}+k_{2}, l_{1}+l_{2}, N}$ from (4.28), Fact 4.6-2 and -4, and Corollary 4.12-(iii), and for the third one this is also clearly true in view of Fact 4.6-4. Since for $\alpha>0$

$$
\partial_{\xi}^{\alpha} f a_{1}=f \partial_{\xi}^{\alpha-1} f\left(\partial_{\xi} a_{1}\right) \in f \sum^{J_{1 / 2}(d)-(\alpha-1)(0,0,1)},
$$

Leibniz rule shows

$$
\partial_{\xi}^{\alpha} f a_{1} \cdot D_{x}^{\alpha}\left(f^{N} b_{2}\right) \in f^{N} \sum^{J_{1 / 2}^{(d)+e}} \text { for any } \alpha,
$$

which would show that the second term also $\in A^{k_{1}+k_{2}, l_{1}+l_{2}, N}$ in view of Corollary 4.12-(iii). This proves the former one, and the latter is similary proved by using Fact 4.6-3 and -7 , and Corollary 4.12-(iii) and (iv). 
(2) We first note that

$$
b \in \cap_{\rho} \sum_{\rho}^{d^{\rho}} \quad \text { with } d^{\rho} \text { satisfying } J_{\rho}\left(d^{\rho}\right)=d,
$$

and that for $k \in \boldsymbol{N}$ we have

$$
\begin{aligned}
& f^{\varepsilon} \partial_{x}^{k}(f b) \in{ }^{f} \Sigma^{d} \quad \text { for } \varepsilon>0, \partial_{x}^{k}\left(f^{f} b\right) \in \bigcap_{\varepsilon>0} \Sigma^{d-(0, \varepsilon, 0)}, \\
& \partial_{\xi}^{k}\left({ }^{f} b\right) \in \bigcap_{1>\varepsilon \geq 0} f^{\varepsilon} \Sigma^{d+2(k-\varepsilon)(0,1,0)} .
\end{aligned}
$$

(4.43) follows from (4.15), (4.41), and that $d_{1}=0$ which follows from $d \in \Delta_{k_{1}, 0}$. The first one of (4.44) are easy consequences of (4.41) and (4.16) which imply that

$$
\begin{gathered}
\left|\partial^{\alpha}{ }^{f} b\right| \leq C_{\alpha}\langle\xi\rangle^{\varepsilon-\alpha_{3}} t^{-\alpha_{1}}\left(\langle f \xi\rangle^{-1 / 2}\right)^{d} f^{f} \psi_{3,1 / 2}{ }^{d_{3}} \\
\text { for all } \alpha \text { and } \varepsilon \in(0,1), \\
\partial_{\xi}^{k} f^{\prime}=f^{\varepsilon}\langle\xi\rangle^{-k+\varepsilon}(f\langle\xi\rangle\langle f \xi\rangle)^{k-\varepsilon} f\left(\langle\xi\rangle^{k-\varepsilon} \partial_{\xi}^{k} b\right) \\
\text { for } \varepsilon \in[0,1) \text { and } k \in N .
\end{gathered}
$$

The second one follows from (4.45), since $d \in \Delta_{k_{1}, 0}$ implies that $\nu(d) \geq 0$, $\mu(d) \geq 0, d_{1}=0$. The last one follows from (4.46), (4.41), (4.3), (4.6), Fact 4.6-7, and that $d \in \Delta_{k_{1}, 0}$ as in the proof of Corollary 4.12-(i).

We may assume that $a={ }^{f} a_{1}+f^{N} b_{1}$ with $a_{1}, b_{1}$ in $\sum^{k_{2}(0,-2,0)}$, for proof of (2), because $\left\langle t^{e}{ } \xi\right\rangle \leq\langle\xi\rangle$ and $d_{1}=0$. (4.41), (4.44), Fact 4.6-2, and Leibniz rule show that for all $k \in N$

$$
\begin{aligned}
& \partial_{\xi}^{k} b_{1} \cdot f^{1 / 2} D_{x}^{k} f b \in \Sigma^{\left(k_{1}+k_{2}-k(2)(0,-2,0)\right.}, \\
& \partial_{\xi}^{k}\left({ }^{f} b\right) \cdot D_{x}^{k}\left(f^{N} b_{1}\right) \in f^{N} \Sigma^{\left(k_{1}+k_{2}-k / 2\right)(0,-2,0)}, \\
& \partial_{\xi}^{k} f \cdot D_{x}^{k f} a_{1}, \partial_{\xi}^{k} a_{1} \cdot D_{x}^{k}\left({ }^{f} b\right) \in{ }^{f} \Sigma^{\left(k_{1}+k_{2}-k / 2\right)(0,-2,0)} .
\end{aligned}
$$

An application of Corollary 4.12-(i) with $\rho=1 / 4$ and of Corollary 4.12-(ii) and -(iv) respectively show that the reminder term $r_{M}$ in the asymptotic expansion (4.18) with $N=M$ of ${ }^{f} b \circ a$ and $a \circ^{f} b$ belong to $B^{0, N}$ for any large $M$, in view of (4.43) and the second one of (4.44). So (4.47) shows (2).

(3) We first note that from (4.42) and (4.16),

$$
\begin{aligned}
& \partial_{x} f a \in \Sigma^{(0,-2,0)}, f \partial_{\xi} a \in \Sigma^{0}, f^{\varepsilon} \partial_{x}^{k} f a \in{ }^{f} \Sigma_{1 / 2}^{(0,-1,1)+(k-1)(0,-1,0)} \\
& \quad \text { for any } \varepsilon>0 \text { and } k \in N
\end{aligned}
$$

To simplify the notation we set

$$
\tilde{r}_{N}[a, b]=r_{N}\left[\partial_{\xi}^{N} a, D_{x}^{N} b\right],
$$




$$
I_{N}[a, b]=\sum_{k=1}^{N-2} \partial_{\xi}^{k} a \cdot D_{x}^{k} b / k !, \quad \text { for } a, b \in \Sigma .
$$

(i) To show the former one we may assume $b={ }^{f} a_{1}+f^{N} b_{1}, a_{1} \in \Sigma_{1 / 2}^{d}, b_{1} \in \Sigma^{e}$, $d \in \Delta^{k, l}, e \in \Delta_{k, l}$. The second one of (4.48) and Fact 4.6-2 and -3 show that

$$
\begin{aligned}
& I_{M}\left[{ }^{f} a,{ }^{f} a_{1}\right] \in{ }^{f} \sum_{1 / 2}^{d+(0,-1,0)}, I_{M}\left[{ }^{f} a, f^{N} b_{1}\right] \in f^{N} \Sigma^{e+(0,-1,0)}, \\
& \tilde{r}_{M}\left[{ }^{f} a_{1},{ }^{f} a\right] \in f^{(M+1) / 2} \sum^{J_{1 / 2}(d)+(0,-1,0)} \\
& \tilde{r}_{M}\left[{ }^{f} a, f^{N} b_{1}\right] \in f^{(M+1) / 2} \sum^{e+(0,-1,0)} \\
& \quad \text { for any } M>0
\end{aligned}
$$

and using the first and third one of (4.48) we see that

$$
\begin{aligned}
& I_{M}\left[{ }^{f} a_{1},{ }^{f} a\right] \in{ }^{f} \sum_{1 / 2}^{d+1 / 2(0,-1,1)}, I_{M}\left[b_{1},{ }^{f} a\right] \in \Sigma^{e+(0,-1,0)}, \\
& \tilde{r}_{M}\left[{ }^{f} a_{1},{ }^{f} a\right] \in f^{M / 2} \Sigma^{f_{1 / 2}(d)+(0,-1,0)}, \tilde{r}_{M}\left[b_{1},{ }^{f} a\right] \in \Sigma^{e+(0,-1,0)}, \\
& \quad \text { for any } M .
\end{aligned}
$$

This shows the former of (i). The latter is shown in the similar way.

(ii) The second of (4.42), Fact 4.6-2, and Leibniz rule show that

$$
\partial_{\xi}^{\alpha} D_{x}^{\alpha} \bar{a} \in{ }^{f} \sum_{1 / 2}^{1 / 2(0,-1,1)} \text { for any } \alpha \in N,
$$

and that

$$
r_{N}^{\sharp}\left[\partial_{\xi}^{N} D_{x}^{N}{ }^{f} \bar{a}\right]=r_{N}^{\sharp}\left[\partial_{\xi}^{N-1} D_{x}^{N-1} \partial_{\xi} D_{x}\left({ }^{f} \bar{a}\right)\right] \in f^{(N-1) / 4} \Sigma^{(0,-1,0)}+\Sigma^{(0, N-1,0)}
$$

from (4.48), Corollary 4.12-(v). This proves (ii).

(iii) (4.42) (4.44) and (4.48) show that

$$
\begin{aligned}
& I_{N}\left[{ }^{f} a,{ }^{f} b\right], I_{N}\left[{ }^{f} b,{ }^{f} a\right] \in{ }^{f} \Sigma^{d}, \\
& \tilde{r}_{N}\left[{ }^{f} a,{ }^{f} b\right] \in f^{(N+1) / 2} \Sigma^{d+(0, N-1-8,0)} \text { for any } \varepsilon, \\
& \tilde{r}_{N}\left[{ }^{f} b,{ }^{f} a\right] \in f^{N / 4} \Sigma^{d+(N / 2-1)(0,1,0)},
\end{aligned}
$$

as in the proof of (2). So (iii) is proved.

(iv) From (4.42) and the last one of (4.48)

$$
\partial_{\xi}^{k}\left({ }^{f} a\right) \cdot D_{x}^{k}\left({ }^{f} b\right) \in^{f} \Sigma_{1 / 2}^{(0,-1,1)} \text { for any } k \in \boldsymbol{N} .
$$

From (4.42) and the first one of (4.48)

$$
\tilde{r}_{N}\left[{ }^{f} a,{ }^{f} b\right] \in f^{(N+1) / 2} \Sigma^{(0,-2,0)} .
$$

This proves (iv).

(4) Let us define inductively $a_{j} \in f^{j / 2} \Sigma_{\rho}^{d-(0,0, j)}$ for $j \in \mathbb{Z}_{+}$so that 


$$
a_{0}=a,
$$

$(*)_{j}$

$$
\begin{gathered}
f^{s}{ }^{f} a_{j}-{ }^{f} a_{j} \circ f^{s}-f^{s}{ }^{f} a_{j+1} \in t^{d_{1}} f^{N} \Sigma^{(0, N, 0)}, \\
a_{j}=f^{j / 2} a_{j}^{\prime} \text { for some } a_{j}^{\prime} \text { in } \Sigma_{\rho}^{d-(0,0, j)}
\end{gathered}
$$

with supp $a_{j}^{\prime} \subset \operatorname{supp} a$.

Assume $a_{i}, 0 \leq i \leq j$ has been chosen. Since $a_{j}=f^{1 / 2} a_{j}^{\prime}$ with $a_{j}^{\prime} \in \Sigma_{\rho}^{d-(0,0, j)}$, and since

$$
k !^{-1} f^{k} D_{x}^{k}\left(f^{s}\right)=g_{k} f^{s+1 / 2} \text { with } g_{k} \in C_{0}^{\infty}(\boldsymbol{R}) \text { for any } k \in \boldsymbol{N},
$$

setting

$$
a_{j+1}^{\prime}=-\sum_{k=1}^{N} g_{k} \partial_{\xi}^{k} a_{j}^{\prime} \quad \text { and } \quad a_{j+1}=f^{(j+1) / 2} a_{j+1}^{\prime}
$$

where

$$
\tilde{N}=4(N+\|d\|+1) \text { with }\|d\|=\sum_{j=1}^{3}\left|d_{j}\right|
$$

we obtain

$$
\begin{aligned}
& f^{s}{ }^{f} a_{j}-{ }^{f} a_{j} \circ f^{s}-f^{s}{ }^{f} a_{j+1}=-\tilde{N} !^{-1} r_{\tilde{N}+1}\left[\partial_{\xi}^{\tilde{N}+1}{ }^{f} a_{j}, D_{x}^{\tilde{N}+1} f^{s}\right], \\
& a_{j+1}^{\prime} \in \Sigma^{d-(0,0, j+1)} \text { with supp } a_{j+1}^{\prime} \subset \operatorname{supp} a
\end{aligned}
$$

so that $(*)_{j}$ holds in view of Corollary 4.12-(ii) and the fact that $a_{j} \in \Sigma^{\mathcal{g}_{p}(d)} \subset$ $t^{d_{1}} \Sigma^{\|d\|(0,-2,0)}$ which follows from Lemma $3.8-2$ and 5. Since $f^{j / 4}=\langle\xi\rangle^{-j / 4}$ $(f\langle\xi\rangle\langle f \xi\rangle)^{j / 4}\langle f \xi\rangle^{j / 4}$, and since $\langle\xi\rangle^{j / 4} \Sigma^{d-(0,0, j)} \subset \Sigma_{\rho}^{d}$, we have

$$
f^{j / 2 f} \sum_{\rho}^{d-(0,0, j)} \subset f^{j / 4} t^{d_{1}} \Sigma^{(|| d||-j / 4)(0,-2,0)}
$$

in view of (4.3), (4.6), Fact 4.6-6. Now by setting $b=\sum_{j=1}^{N} a_{j}$ and by using $(*)_{j}$,

$$
f^{s} \cdot{ }^{f} a-a \circ f^{s}-{ }^{f} b \circ f^{s}-f^{s} \cdot f_{\tilde{N}^{+1}} \in f^{N} t^{d_{1}} \Sigma^{(0, N, 0)}
$$

which proves the former of (4) in view of

$$
\Sigma^{(\|d\|-(\tilde{N}+1) / 4)(0,-2,0)} \subset \Sigma^{(0, N, 0)} .
$$

The latter is similar.

(4)' By the asymptotic expansion, for any $a \in \Sigma_{1 / 2}^{d}$ (resp. $\Sigma^{d}$ ) and $s \geq 0$,

$$
{ }^{f} a \circ f^{s}=\sum_{j \leq \mathbb{L}}{ }^{f}\left(\partial^{j} a\right) f^{j} D_{x}^{j}\left(f^{s}\right) / j !+M !^{-1} r_{M+1}\left[\partial_{\xi}^{M+1}{ }^{f} a, D_{x}^{M+1} f^{s}\right] .
$$

The summation can be written as $f^{s}\left({ }^{f} a+{ }^{f} b\right)$ with $b \in \Sigma_{1 / 2}^{d-(0,0,1)}$ (resp. $\left.\Sigma^{d-(0,0,1)}\right)$ satisfying supp $b \subset \operatorname{supp} a$ as in the proof of (4). Since $\sum_{1 / 2}^{d} \subset \Sigma^{\mathscr{I}_{\mathrm{p}}(d)}$ (resp. 
$\left.\Sigma^{d} \subset \Sigma^{\mathscr{g}(d)}\right)$, the last term is in

$$
f^{(M+1) / 2} \Sigma^{\mathcal{G}_{\mathrm{p}}(d)+(M+1)(0,1,0)}\left(\text { resp. } f^{(M+1) / 2} \sum^{\mathscr{g}(d)+(M+1)(0,1,0)}\right)
$$

by Corollary 4.12-(ii). By Lemma 3.8-2 and -5 for any $N$ there exists $M \in \mathbb{N}$ such that the last term is in

$$
f^{N} t^{d_{1}} \Sigma^{(0, N, 0)}\left(\operatorname{resp} . f^{N} t^{d_{1}} \Sigma^{(0, N, 0)}\right) .
$$

This completes the proof.

(5) The proof is similar to that of (4). One can define inductively $a_{j}, j=1, \ldots$, $\tilde{N}=N+3(\|d\|+1)+1$, in $\Sigma^{d-(0,0, j)}$ so that

$$
\begin{aligned}
& a_{0}=a, \\
& f^{s_{j}} O p a_{j}-\left(O p a_{j}\right) f^{s_{j}}-f^{s_{j+1}} O p a_{j+1} \in t^{d_{1}} \text { Op } \Sigma^{(0, N, 0)}, 0 \leq j \leq \tilde{N}-1 \\
& \text { where } s_{j}=s-j \varepsilon /(\tilde{N}+1) \text {. }
\end{aligned}
$$

Then setting $b=-\sum_{j=1}^{\tilde{N}-1} a_{j} \circ f^{s_{j}-s+\varepsilon}$ we have

$$
\begin{aligned}
& b \in \Sigma^{d-(0,0,1)}, \\
& f^{s} O p a-(O p a) f^{s}+(O p b) f^{s-\varepsilon}-f^{s} \tilde{N} O p \quad a_{\tilde{N}} \in t^{d_{1}} O p \Sigma^{(0, N, 0)}
\end{aligned}
$$

which show the assertion. The proof is complete.

Lemma 4.19. Let $\|\cdot\|=\|\cdot\|_{L^{2}\left(\boldsymbol{R}_{x}\right)}$ 。

(1) Let $a \in \Sigma_{1 / 2}^{d}(T, h)$ with $\sigma_{1 / 2}(d)=k, d_{1}=-2 l, l \geq 0$, and $\mu_{1 / 2}(d) \geq 0$. Then, there exists $C>0$ such that for any $u \in \mathcal{S}_{T}$

$$
\left\|\left(\left(O p{ }^{f} a\right) u\right)_{t}\right\| \leq C t^{-2 l}\left(t^{e}{ }^{l}\left\|\Lambda_{k} f^{1 /(10 h)} u_{t}\right\|+\left\|\Lambda_{k-1} u_{t}\right\|+\left\|u_{t}\right\|\right), t \in(0, T) .
$$

(2) Let $a \in \Sigma^{d}(T, h)$ with $\sigma(d)=k, d_{1}=-2 l, l \geq 0$, and $\tilde{\mu}(d) \geq 0$. Let $s \geq 0$. Then, there exists $C>0$ such that for any $u \in \mathcal{S}_{T}$,

$$
\begin{aligned}
& \left\|f^{s+\max (\sigma(d), 0)}((O p a) u)_{t}\right\| \leq C t^{-2 l}\left(t^{e_{h}}\left\|\Lambda_{k} f^{s} u_{t}\right\|+\left\|\Lambda_{k-l} u_{t}\right\|+\left\|u_{t}\right\|\right), \\
& t \in(0, T)
\end{aligned}
$$

(3) Let $a \in \Sigma^{d}(T, h)$ and $s \geq 0$ be as in (2). Then there exists $C>0$ such that for any $u \in \mathcal{S}_{T}$,

$$
\left\|f^{s}\left(\left(O p{ }^{f} a\right) u\right)_{t}\right\| \leq C t^{-2 l}\left(t^{e_{h}}\left\|\Lambda_{k} f^{s} u_{t}\right\|+\left\|\Lambda_{k-l} u_{t}\right\|+\left\|u_{t}\right\|\right), t \in(0, T) .
$$

Proof. (1) Since $\mu_{1 / 2}(d)+d_{3}=k-l$ and $\mu_{1 / 2}(d) \geq 0,\left\langle t^{e}{ }_{h \xi}\right\rangle^{\mu_{1 / 2}{ }^{(d)}}\langle\xi\rangle^{d_{3}} \leq$ $\langle\xi\rangle^{k-l}$ so that $a \in \Sigma_{1 / 2}^{-2 l, 0,0)+(k-l)(0,-1,1)}$. So, by applying Lemma 4.13-(1) with $u_{t}$ for $u$ and with $(-2 l, 0,0)+(k-l)(0,-1,1)$ for $d$ and by estimating the term $\left\|f^{l /(10 h)} \Lambda_{k} u_{t}\right\|$ by Corollary $4.3-(1)$ one obtain the desired inequality. 
(2) From Lemma 4.13-(4) and Corollary 4.3-(1) one can easily see that for $A \in$ $f^{\max (k, 0)} O p \Sigma^{(-2 l,-2(k-l), 0)}$ and $s^{\prime} \geq s \geq 0$, there exists $C>0$ such that for $u \in \mathcal{S}_{T}$,

$$
\left\|\left(A f^{s^{\prime}} u\right)_{t}\right\| \leq C t^{-2 l}\left(t^{e h^{l}}\left\|\Lambda_{k} f^{s} u_{t}\right\|+\left\|\Lambda_{k-l} u_{t}\right\|+\left\|u_{t}\right\|\right), t \in(0, T) .
$$

As in the proof of (1) we have $a \in \Sigma^{(-2 l,-2(k-l), 0)}$ from $\tilde{\mu}(d) \geq 0$ and $k-l=\tilde{\mu}(d)$ $+\nu(d)$. When $s=0$, (4.51) with $s^{\prime}=s=0$ implies (2). When $s>0$, from Lemma 4.18-(5) and the uniqueness of the symbol,

(4.52) for $\varepsilon \in(0, s)$ there exists $b \in \Sigma^{d-(0,0,1)}, r \in t^{-2 l} \Sigma^{(0,1,0)}$ satisfying $f^{s} a-a \circ f^{s}-b \circ f^{s-\varepsilon}=r$.

We consider separately two subcases: (i) $\max (k, 0) \leq 1 / 2$, (ii) $\max (k, 0)>1 / 2$. In (i), we have $b \in t^{-2 l} \Sigma^{0}$ so that from (4.52) one can derive (2) by using (4.51) with $s^{\prime}=s$ and with $f^{\max (k, 0)} O p a$ for $A$. In (ii), again Lemma 4.18-(5) implies that

$$
\begin{aligned}
& \text { for } \varepsilon \in(0,1 / 2) \text { there exists } b_{1} \in \Sigma^{d-(0,0,2)} \text { and } r_{1} \in t^{-2 l} \Sigma^{(0,1,0)} \\
& \text { satisfying } f^{1 / 2} b-b \circ f^{1 / 2}-b_{1} \circ f^{1 / 2-\varepsilon_{1}}=r_{1} .
\end{aligned}
$$

Take $\varepsilon$ and $\varepsilon_{1}$ in (4.52) and (4.53) as $\varepsilon+\varepsilon_{1}<1 / 2$ so that setting $\varepsilon^{\prime}=1 / 2-\varepsilon$ and $\varepsilon^{\prime \prime}=1 / 2-\varepsilon-\varepsilon_{1}$, we have

$$
f^{k+s} a-f^{k} a \circ f^{s}-f^{k-1 / 2} b \circ f^{s+\varepsilon^{\prime}}-f^{k-1 / 2} b_{1} \circ f^{s+\varepsilon^{\prime \prime}}=f^{k} r+f^{k-1 / 2} r_{1} .
$$

Since $b, b_{1} \in \Sigma^{(-2 l,-2(k-1 / 2-l), 0)}$ from $\tilde{\mu}(d) \geq 0$, one would obtain (2) by applying (4.51) with $O p f^{k} a, O p f^{k-1 / 2} b$, and $O p f^{k-1 / 2} b_{1}$ respectively for $A$ and using Lemma 4.1-(1) to estimate the terms $\left\|\Lambda_{k-1 / 2} f^{s} u_{t}\right\|$ and $\left\|\Lambda_{k-1 / 2-l} u_{t}\right\|$. This completes the proof of (2).

(3) The proof is similar to that of (2). From Lemma 4.18-(4), there exists $b \in \Sigma^{d-(0,0,1)}$ and $r \in \Sigma^{(0,1,0)}$ such that $f^{s}{ }^{f} a-{ }^{f} a \circ f^{s}-{ }^{f} b \circ f^{s}=t^{-2 l} r$. From $\tilde{\mu}(d)$ $\geq 0$ and $k-l=\tilde{\mu}(d)+\nu(d)$ we have that $a, b \in \Sigma^{(-2 l,-2(k-l), 0)}$. Then, Lemma 4.13-(2) and Corollary 4.13-(1) shows (3). The proof is complete.

Now we recall the definition of a norm $\|\cdot\|^{(n)}$ on $\mathcal{S}_{T}$.

Definition 4.20. For $s=0, \cdots, 6$ and $n \geq 1$ we define norms on $\mathcal{S}_{T}$ :

$$
\begin{aligned}
& \|u\|_{(s)}^{(n)}=\sum_{i+2 j \leq s} n^{3 / 2-i / 2-j}\left\|\Lambda_{i / 2} D_{t}^{j} u\right\|^{(n+3-i / 2-j)} \\
& \|u\|_{(s, n)}=\sum_{\substack{i+2 j \leq s \\
i, j \in Z_{+}}}\left\|\Lambda_{i / 2} D_{t}^{j} u\right\|^{(n)}
\end{aligned}
$$

It is easy to see that 


$$
\begin{aligned}
& \|u\|_{(s)}^{(n)} \leq\|u\|_{\left(s^{\prime}\right)}^{(n)},\|u\|_{(s, n)} \leq\|u\|_{\left(s^{\prime}, n\right)}, \text { if } \quad s<s^{\prime}, \\
& \|u\|^{(n)} \leq T^{\alpha}\|u\|^{(n+\alpha)},\|u\|_{(s)}^{(n)} \leq\left(\frac{1+\alpha}{\alpha}\right)^{-3 / 2} T^{\infty}\|u\|_{(s)}^{(n+\alpha)}, \\
& \|u\|_{(s, n)} \leq\|u\|_{(s, n+\alpha),}, \text { for } \alpha>0 .
\end{aligned}
$$

Lemma 4.21. (1) Let $A \in \mathscr{B}^{k, l, N}$. Then there exists $C>0$ such that

$$
\|A u\|^{(n)} \leq C\left(\left\|\Lambda_{k} u\right\|^{(n+l(1-1 /(10 h))}+\left\|\Lambda_{k-l} u\right\|^{(n+2 l)}+\|u\|^{(n+2 l)}\right) .
$$

(2) Let $A \in \mathscr{B}^{k, l, N}$ with $s / 2+k \leq N$. Then there exists $C>0$ such that

$$
\begin{aligned}
\|A u\|_{(s)}^{(n) \leq} \leq & C\left(\left\|\Lambda_{k} u\right\|_{(s)}^{(n+l(1-1 /(10 h))}+\left\|\Lambda_{k-l} u\right\|_{(s)}^{(n+2 l)}+\|u\|_{(s)}^{(n+2 l)}\right) \\
\|A u\|_{(s, n)} \leq & C \sum_{i=0}^{s}\left(\left\|\Lambda_{k} u\right\|_{(s-i, n+l(1-1 /(10 h))+i / 2)}\right. \\
& \left.+\left\|\Lambda_{k-l} u\right\|_{(s-i, n+2 l+i / 2)}+\|u\|_{(s-i, n+2 l+i / 2)}\right) .
\end{aligned}
$$

(3) Let $\alpha \in R$. Then there exists $C>0$ such that for $n \geq \max (0, \alpha)+1$,

$$
\begin{aligned}
& \left\|t^{\infty} u\right\|_{(s, n)} \leq C\|u\|_{(s, n-\alpha)}, \\
& \left\|t^{\infty} u\right\|_{(s)}^{(n)} \leq C\|u\|_{(s)}^{(n-\alpha)} .
\end{aligned}
$$

(4) Let $a \in B_{T}$. Then there exists $C>0$ such that

$$
\|a u\|_{(s, n)} \leq C\|u\|_{(s, n)} .
$$

Proof. (1) We may assume that $A=O p\left({ }^{f} a+f^{N} b\right), a \in \Sigma^{d}, b \in \Sigma^{e}$ with $d, e \in \Delta_{k, 1}$. Since $\sigma(\tilde{d})=k-l, \tilde{\mu}(\tilde{d}) \geq 0, t^{-1}\left\langle t^{e} h \xi\right\rangle^{1 / 2} \geq\langle\xi\rangle^{1 / 2}$, and $\tilde{d}_{1} \geq-2 l$ when $\tilde{d} \in \Delta_{k, 1}$, we see $t^{\tilde{d}_{1}}\left\langle t^{e}{ }^{e} \xi\right\rangle^{-\tilde{d}_{1} / 2+\tilde{\mu}(\tilde{d})}\langle\xi\rangle^{v(\tilde{d})} \leq\left(t^{-1}\left\langle t^{e} h \xi\right\rangle^{1 / 2}\right)^{2 l}\langle\xi\rangle^{k-l}$. So $a, b \in$ $\Sigma^{(-2 l,-2(k-l), 0)}$. Thus (1) is an immediate consequence of Lemma 4.19-(2), -(3). (2) We may assume $A=O p\left({ }^{f} a+f^{N} b\right)$ with $a, b \in \Sigma^{(0,-2 k, 0)}$ from the proof of (1). We set

(4.56) $A^{(l)}=O p\left(D_{t}^{l} a\right)$ if $A=O p a$, by definition.

Then by using Lemma 4.18-(1),

$$
\begin{aligned}
& \Lambda_{i / 2} D_{t}^{j} A=\sum_{m \leq j} \Lambda_{i / 2} A^{(j-m)} D_{t}^{m}, \quad \text { and } \\
& \Lambda_{i / 2} A^{(j-l)} \in \mathscr{B}^{k+(i+j-m) / 2,(j-m) / 2+l, N} .
\end{aligned}
$$

Now by using this, (1), and Lemma 4.2-(2), (2) is proved in a straightforward way.

(3) A simple consequence of Leibniz rule and (1). This completes the proof.

Lemma 4.22. Let $A \in \mathscr{A}^{k, l, N}\left(\right.$ resp. $\left.\mathscr{B}^{k, l_{0} N}\right)$. Then if $N \geq k+j / 2, A^{(j)} \in$ $\mathscr{A}^{k+j / 2, l+j / 2, N}$ (resp. $\mathscr{B}^{k+j / 2, b+j / 2, N}$ ). In particular $\left[D_{t}, A\right] \in \mathcal{A}^{k+1 / 2, l+1 / 2, N}$ (resp. 
$\mathcal{B}^{k+1 / 2, l+1 / 2, N}$ ) when $N \geq k+1 / 2$.

This lemma is easy.

\section{§. The Basic Estimates}

Let $T^{(0)} \in(0,1), a \in B_{h, T}(0)$, and $\tilde{\sigma} \in t^{8} \Sigma_{1 / 2}^{(0,-1,1)}\left(T^{(0)}, h\right)$ with $\varepsilon \in(0,1)$ satisfying

$$
\partial_{\xi} \tilde{\sigma} \in t^{\varepsilon} \Sigma_{1 / 2}^{0}, \partial_{x} \tilde{\sigma} \in t^{\varepsilon} \Sigma_{1 / 2}^{(0,-1,1)}, \partial_{t} \tilde{\sigma} \in t^{\varepsilon-1} \Sigma_{1 / 2}^{(0,-1,1)},
$$

(5.2) $\inf |\operatorname{Im} a|>0$,

and set

$$
L=D_{t}-O p(a f \xi+\sigma), \sigma={ }^{f} \tilde{\sigma} .
$$

Then the following proposition is fundamental for the proof of Proposition 2.3.

Proposition 5.1. There exists $C>0$ and $T_{0} \leq T^{(0)}$ such that for $T \in\left(0, T_{0}\right)$ and $u \in \mathcal{S}_{T}, n \geq 1$,

$$
\|L u\|^{(n)} \geq C\left\{n^{-1 / 2}\left(\left\|D_{t} u\right\|^{(n)}+\left\|\Lambda_{1} u\right\|^{(n)}\right)+n^{1 / 2}\|u\|^{(n+1)}\right\} .
$$

Proof. Let $T \in\left(0, T^{(0)}\right)$ and $u \in \mathcal{S}_{T}$, and set

$$
\begin{aligned}
& A_{1}=O p(\operatorname{Re}(a f \xi+\sigma)), A_{2}=O p(\operatorname{Im}(a f \xi+\sigma)), \\
& L_{1}=D_{t}-A_{1}, L_{2}=A_{2}+n t^{-1} .
\end{aligned}
$$

Then, since $t^{-n} L u=L_{1} v-i L_{2} v$, setting $v=t^{-n} u, n \geq 1$ we have

$$
\begin{aligned}
& \left(\|L u\|^{(n)}\right)^{2}=\left\|L_{1} v\right\|^{2}+J(v)+\left\|L_{2} v\right\|^{2}, \\
& J(v)=-2 \operatorname{Im}\left(L_{1} v, L_{2} v\right),
\end{aligned}
$$

where $\|\cdot\|=\|\cdot\|_{L^{2}((0, T) \times R)}$ and $()=,(,)_{L^{2}((0, T) \times R)}$. Let us show that

(5.5) there exists $T_{0} \in\left(0, T^{(0)}\right)$ such that for any $T \in\left(0, T_{0}\right), n \geq 1, v \in \mathcal{S}_{T}$, $J(v) \geq n / 2\left\|t^{-1} v\right\|^{2}-1 / 5\left(\left\|L_{1} v\right\|^{2}+\left\|L_{2} v\right\|^{2}\right)$.

To do so we first note that

(5.6) $2 i \operatorname{Im}\left(L_{1} v, L_{2} v\right)=\left(v,\left[L_{1}, L_{2}\right] v\right)+\left(\left(L_{1}-L_{1}^{*}\right) v, L_{2} v\right)+\left(\left(L_{2}^{*}-L_{2}\right) v, L_{1} v\right)$.

Next noting

$$
\begin{aligned}
& L_{1}^{*}-L_{1}=A_{1}-A_{1}^{*}, L_{2}^{*}-L_{2}=A_{2}^{*}-A_{2}, \\
& {\left[L_{1}, L_{2}\right]=\left[D_{t}, A_{2}\right]-\left[A_{1}, A_{2}\right]+i n t^{-2},}
\end{aligned}
$$


and using Lemma 4.18-(ii) and -(iv) and (5.1) we see, in view of $\varepsilon \in(0,1)$,

$$
L_{i}^{*}-L_{i} \in \mathcal{A}^{1 / 2,1 / 2}+\text { Op } \Sigma^{0},\left[L_{1}, L_{2}\right]-\mathrm{int}^{-2} \in t^{\varepsilon-1} \mathcal{A}^{1,1} .
$$

From the latter of (5.7),

$$
\begin{aligned}
S_{n} & \equiv i\left(2 \operatorname{Im}\left(L_{1} v, L_{2} v\right)-n\left\|t^{-1} v\right\|^{2}\right) \\
& =\left(t^{t^{-1}} v, A v\right)+\left(\left(L_{1}-L_{1}^{*}\right) v, L_{2} v\right)+\left(\left(L_{2}^{*}-L_{2}\right) v, L_{1} v\right) \\
& \text { for some } A \in \mathcal{A}^{1,1} .
\end{aligned}
$$

So, estimating $\|A v\|,\left\|\left(L_{i}-L_{i}^{*}\right) v\right\|$ by (5.7) and Lemma 4.29-(1), -(2), and using $\|v\| \leq\left\|t^{8-1} v\right\|$ we see that there exists $C_{1}>0$ such that for $T \in\left(0, T^{(0)}\right), n \geq 1$, $v \in \mathcal{S}_{T}$,

$$
\left|S_{n}\right| \leq C_{1}\left(\left\|\Lambda_{1} v\right\|\left\|t^{\varepsilon-1} v\right\|+\left\|\Lambda_{1 / 2} v\right\|^{2}+\|v\|\left\|t^{\mathrm{g}-1} v\right\| \mid\right)+\sum_{i=1}^{2}\left\|L_{i} v\right\|^{2} / 10 .
$$

On the other hand, (5.2) and Lemma 4.1-(i) show that there exist $C_{2}, C_{3}>0$ such that for $T \in\left(0, T^{(0)}\right), v \in \mathcal{S}_{T}, t \in(0, T)$,

$$
\begin{aligned}
& \left\|\operatorname{Im} a(t, \cdot) f D_{x} v(t, \cdot)\right\|_{0} \geq C_{2}\left\|A_{1} v(t, \cdot)\right\|_{0}-C_{3}\|v(t, \cdot)\|_{0} \\
& \text { where }\|\cdot\|_{0}=\|\cdot\|_{L^{2}\left(\boldsymbol{R}_{x}\right)} .
\end{aligned}
$$

Thus, since $\tilde{\sigma} \in t^{\varepsilon} \Sigma^{(0,-1,1)}$ with $\varepsilon>0$ from Lemma 4.29-(1), there exists $T_{1} \in$ $\left(0, T^{(0)}\right)$ and $C_{4}>0$ such that for $T \in\left(0, T_{1}\right), v \in \mathcal{S}_{T}, t \in(0, T)$,

$$
\left\|\left(A_{2} v\right)(t, \cdot)\right\|_{0} \geq C_{0} / 2\left\|\Lambda_{1} v(t, \cdot)\right\|_{0}-C_{4}\|v(t, \cdot)\|_{0}
$$

which means that there exists $C_{5}>0$ such that

$$
\begin{aligned}
& \text { for } \quad T \in\left(0, T_{1}\right), v \in \mathcal{S}_{T}, t \in(0, T), \\
& \left\|\Lambda_{1} v\right\| \leq C_{5}\left(\left\|A_{2} v\right\|+\|v\|\right) .
\end{aligned}
$$

Since $\left\|\Lambda_{1 / 2} v\right\|^{2}=\left(\Lambda_{1 / 2} * \Lambda_{1 / 2} v, v\right)$, and since by noting $\langle f \xi\rangle^{1 / 2} \in S_{1,0}^{1 / 2}(\boldsymbol{R})$ from (4.3), the asymptotic expansion the symbol of $\Lambda_{1 / 2}{ }^{*}$ shows by breaking off after 2 terms, that

$$
\Lambda_{1 / 2}{ }^{*}={ }^{f} a(x, D)+b(x, D) \text { with } a \in S_{1,0}^{1 / 2} \text { and } b \in S_{1,1 / 2}^{-1 / 2},
$$

one can obtain using Lemma 4.2-(ii) and 4.1-(i) that there exists $C_{6}>0$ such that

$$
\left\|\Lambda_{1 / 2} v\right\|^{2} \leq C_{6}\left(\left\|\Lambda_{1} v\right\|+\|v\|\right)\|v\| .
$$

From (5.9) (5.11) it follows by using $\|v\| \leq\left\|t^{8-1} v\right\|$ that there exists $C_{7}>0$ such that for $T \in\left(0, T_{1}\right), v \in \mathcal{S}_{T}, t \in(0, T), n \geq 1$, 


$$
\left|S_{n}\right|-\sum_{i=1}^{2}\left\|L_{i} v\right\| / 10 \leq C_{7} T^{\varepsilon}\left(\left\|A_{2} v\right\|\left\|t^{-1} v\right\|+\left\|t^{-1} v\right\|^{2}\right) .
$$

Now using this and

$$
\left\|A_{2} v\right\| \leq\left\|L_{2} v\right\|+n\left\|t^{-1} v\right\|, v \in \mathcal{S}_{T}
$$

we obtain that there exists $T_{0} \in\left(0, T_{1}\right)$ such that for $T \in\left(0, T_{0}\right), v \in \mathcal{S}_{T}$,

$$
\left|S_{n}\right| \leq \sum_{i=1}^{2}\left\|L_{i} v\right\|^{2} / 5+\left\|t^{-1} v\right\|^{2} / 2 \text {. }
$$

This shows (5.5) which implies that for $T \in\left(0, T_{0}\right), u \in \mathcal{S}_{T}, n \geq 1$,

$$
\left(\|L u\|^{(n)}\right)^{2} \geq n\left\|t^{-1} v\right\|^{2} / 2+4 \sum_{i=1}^{2}\left\|L_{i} v\right\|^{2} / 5 .
$$

Finally we observe that there exists $C_{8}>0$ such that for $T \in\left(0, T^{(0)}\right), u \in \mathcal{S}_{T}$, $n \geq 1$,

$$
\left\|D_{t} u\right\|^{(n)} \leq\left\|L_{1} v\right\|+C_{8}\left\|\Lambda_{1} v\right\|+n\left\|t^{-1} v\right\| .
$$

(5.10), (5.12), (5.14), (5.15) show the desired inequlity. This completes the proof.

Let $L_{i}=D_{t}-O p\left(a_{i} f \xi+\sigma_{i}\right), i=1,2,3$ be three operators as in (5.3) for fixed $\varepsilon$. We define

$$
\begin{aligned}
& M_{n}^{\prime}(u)=M_{n}\left(u, L_{i}\right)=\left\|L_{1} L_{2} u\right\|^{(n)}+\left\|L_{2} L_{1} u\right\|^{(n)}, \\
& M_{n}(u)=M_{n}\left(u, L_{i}\right)=\sum_{\sigma \in \mathcal{G}_{3}}\left\|L_{\sigma(1)} L_{\sigma(2)} L_{\sigma(3)} u\right\|^{(n)},
\end{aligned}
$$

for $u \in \mathcal{S}_{T}, n \geq 1$ where $\mathfrak{S}_{3}$ denotes the symmetric group of degree 3 .

Corollary 5.2. There exists $C>0$ and $T_{0} \in\left(0, T^{(0)}\right)$ such that for $n \geq 1$, $T \in\left(0, T_{0}\right), u \in \mathcal{S}_{T}$.

(i) $n\|u\|^{(n+2)}+n^{-1 / 2} \sum_{i=1}^{2}\left(\left\|D_{t} L_{i} u\right\|^{(n)}+\left\|\Lambda_{1} L_{i} u\right\|^{(n)}\right)+n^{-1} \sum_{k=0}^{2}\left\|\Lambda_{2-k} D_{t}^{k} u\right\|^{(n)}$

$$
\leq C M_{n}^{\prime}(u)
$$

(ii) $n^{3 / 2}\|u\|^{(n+3)}+n^{-1 / 2} \sum_{i \neq j}\left(\left\|D_{t} L_{i} L_{j} u\right\|^{(n)}+\left\|\Lambda_{1} L_{i} L_{j} u\right\|^{(n)}\right)$

$$
+n^{-1} \sum_{i=1}^{3} \sum_{k=0}^{2}\left\|\Lambda_{k} D_{t}^{2-k} L_{i} u\right\|^{(n)}+n^{-2 / 3} \sum_{k=0}^{3}\left\|\Lambda_{3-k} D_{t}^{k} u\right\|^{(n)} \leq C M_{n}(u)
$$

Proof. For the sake of simplicity we denote by $C$ or $C_{i}$ a constant indentdepen of $T, n, u \in \mathcal{S}_{T}$. We only prove that Proposition 5.1 and (i) imply (ii). the similar way one can prove (i) assuming Proposition 5.1. Exept the inequality

$$
S \equiv n^{-3 / 2} \sum_{k=0}^{3}\left\|\Lambda_{3-k} D_{t}^{k} u\right\|^{(n)} \leq C M_{n}(u),
$$


the validity of the others immediately follows from the assumption. So, it suffices to prove only (5.17) assuming all other inequalities in (ii), and those in (i), Proposition 5.1, for any $T \in\left(0, T_{1}\right)$ with uniform consiant $C$ with some $T_{1} \in\left(0, T^{(0)}\right)$. Using the assumption and applying Corollary 4.3-(2) to $\Lambda_{2} \Lambda_{1}$ we see that

$$
\begin{aligned}
S & \leq n^{-3 / 2}\left\{\sum_{k=0}^{2}\left\|\Lambda_{2-k} D_{t}^{k+1} u\right\|+C_{1}\left(\left\|\Lambda_{2} \Lambda_{1} u\right\|^{(n)}+\|u\|^{(n)}\right)\right\} \\
& \leq C_{2}\left\{n^{-1 / 2}\left(M_{n}^{\prime}\left(D_{t} u\right)+M_{n}^{\prime}\left(\Lambda_{1} u\right)\right)+M_{n}(u)\right\} \text { for } T<T_{1} .
\end{aligned}
$$

We note that from Lemma 4.18-(3)-(iv) and (5.1)

$$
\left[L_{i}, D_{t}\right] \in t^{\varepsilon-1} \mathcal{A}^{1,2},\left[L_{i}, \Lambda_{1}\right] \in \mathcal{A}^{1,2},
$$

and that from Lemmas 4.18-(3)-(i) and 4.22,

$$
\begin{aligned}
& {\left[L_{i},\left[L_{j}, D_{t}\right]\right] \in t^{\varepsilon-1} \mathscr{A}^{3 / 2,1 / 2,3 / 2}+t^{\varepsilon-2} \mathscr{A}^{1,2},} \\
& {\left[L_{i},\left[L_{j}, \Lambda_{1}\right]\right] \in \mathscr{A}^{3 / 2,1 / 2,2} .}
\end{aligned}
$$

Therefore, since

$$
\begin{aligned}
& {\left[L_{i} L_{j}, D_{t}\right]=\left[L_{i},\left[L_{j}, D_{t}\right]\right]+\left[L_{j}, D_{t}\right] L_{i}+\left[L_{i}, D_{t}\right] L_{j},} \\
& {\left[L_{i} L_{j}, \Lambda_{1}\right]=\left[L_{i},\left[L_{j}, \Lambda_{j}\right]\right]+\left[L_{j}, \Lambda_{1}\right] L_{i}+\left[L_{i}, \Lambda_{1}\right] L_{j},}
\end{aligned}
$$

we see by Lemma $4.21-(1)$,

$$
\begin{aligned}
& \left\|L_{i} L_{j} D_{t} u\right\|^{(n)} \leq C_{3}\left\{\left\|\Lambda_{3 / 2} u\right\|^{(n+3 / 2-\varepsilon)}+\left\|\Lambda_{1} u\right\|^{(n+2-\varepsilon)}+\|u\|^{(n+2-\varepsilon)}\right. \\
& \left.\quad+\sum_{1 \in\{i, j\}}\left(\left\|\Lambda_{1} L_{l} u\right\|^{(n+1-\varepsilon)}+\left\|L_{l} u\right\|^{(n+1-\varepsilon)}\right)\right\}+\left\|D_{t} L_{i} L_{j} u\right\|^{(n)} \\
& \quad \text { for } T<T_{1} \\
& \left\|L_{i} L_{j} \Lambda_{1} u\right\|^{(n)} \leq C_{3}\left\{\left\|\Lambda_{3 / 2} u\right\|^{(n+1 / 2)}+\left\|\Lambda_{1} u\right\|^{(n+1)}+\|u\|^{(n+1)}\right. \\
& \left.\quad+\sum_{l \in\{i, j\}}\left(\left\|\Lambda_{1} L_{l} u\right\|^{(n)}+\left\|L_{l} u\right\|^{(n)}\right)\right\}+\left\|\Lambda_{1} L_{i} L_{j} u\right\|^{(n)} \\
& \quad \text { for } T<T_{1} .
\end{aligned}
$$

Applying Lemma 4.1-(1) with $\varepsilon=t^{-1}$ and using the assumption we see that

$$
\begin{aligned}
& \left\|\Lambda_{3 / 2} u\right\|^{(n+3 / 2-\varepsilon)}+\left\|\Lambda_{1} u\right\|^{(n+2-\varepsilon)}+\|u\|^{(n+2-\varepsilon)} \\
& \quad \leq C_{4} T^{\varepsilon}\left(n^{-3 / 2}\left\|\Lambda_{3} u\right\|^{(n)}+M_{n}(u)\right) \text { for } T<T_{1} .
\end{aligned}
$$

(5.18) (5.21) and the assumption imply that if $T<T_{1}$,

$$
S \leq C_{5}\left(T^{\varepsilon} n^{-3 / 2}\left\|\Lambda_{3} u\right\|^{(n)}+M_{n}(u)\right) .
$$

This proves (5.17) for sufficiently small $T$. The proof is complete. 


\section{§6. Lemmas on the Commutation}

We first study commutations involving in operators as in (5.3). We prepare three lemmas.

Lemma 6.1. Let $a_{i} \in C^{\infty}\left(\boldsymbol{R}_{y} \times \boldsymbol{R}_{\eta}\right), i=1,2,3$ such that for some $M>0$ and $\delta \in[0,1)$,

$$
\left|\partial_{y}^{\alpha} \partial_{\eta}^{\beta} a_{i}(y, \eta)\right| \leq C_{\alpha \beta}\langle\eta\rangle^{M+\delta|\alpha|} \text { for all } \alpha, \beta .
$$

Then, we have the followings.

(i) If $a_{2}$ is constant in $\eta$,

$$
\begin{aligned}
O s & -\iint e^{-i y \eta} a_{1}(y, \eta) a_{2}(y) d y d \eta \\
& =\iint e^{-i y \eta}\left(a_{1}(y, \eta) a_{2}(0)+D_{\eta} a_{1}(y, \eta) \int_{0}^{1}\left(\partial_{y} a_{2}\right)(\theta y) d \theta\right) d y d \eta .
\end{aligned}
$$

(ii) If $a_{1}$ and $a_{2}$ are constant in $y$,

$$
\begin{aligned}
O s & -\iint e^{-i y \eta} a_{1}(\eta) a_{2}(\eta) a_{3}(y, \eta) d y d \eta \\
& =O S-\iint e^{-i y \eta}\left(a_{1}(0) a_{2}(0) a_{3}(y, \eta)+\int_{0}^{1}\left(\partial_{\eta} a_{1}\right)(\theta \eta) d \theta \cdot a_{2}(0) D_{y} a_{3}(y, \eta)\right. \\
& \left.+a_{1}(0) \int_{0}^{1}\left(\partial_{\eta} a_{2}\right)(\theta \eta) d \theta \cdot D_{y} a_{3}(y, \eta)+\prod_{i=1}^{2} \int_{0}^{1}\left(\partial_{\eta} a_{i}\right)(\theta \eta) d \theta \cdot D_{y}^{2} a_{3}(y, \eta)\right) d y d \eta .
\end{aligned}
$$

This is an immediate consequence of the integration by parts. We omit the proof.

Lemma 6.2. For $d^{1}, \cdots, d^{k} \in \boldsymbol{R}^{3}$ with $d_{1}^{j} \leq 0$ for any $j$, we denote by $S$

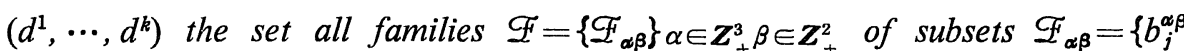
$\left.(t, x, y, \xi, \eta) \in C^{\infty}\left(\boldsymbol{R}_{T}^{5}\right), j=1, \cdots, n(\alpha, \beta)\right\} \cup\left\{\lambda^{i, j} \in \boldsymbol{Z}_{+}^{3}, \mu^{i, j} \in \boldsymbol{Z}_{+}^{2}, i=1, \cdots, k\right.$, $\left.j=1, \cdots, n(\alpha, \beta) ; \sum_{i=1}^{k} \lambda^{i, j}=\alpha, \sum_{i=1}^{k} \mu^{i, j}=\beta\right\}$ where $n(\alpha, \beta) \in \boldsymbol{N}$ such that for any $r \in Z_{+}^{2}$ there exists $C_{\alpha \beta \gamma}>0$ such that for $j=1, \cdots, n(\alpha, \beta)$

$$
\begin{aligned}
& \left|\partial_{y, \eta}^{\gamma} b_{j}^{\alpha \beta}(t, x, y, \xi, \eta)\right| \leq C_{\alpha \beta \gamma} t \sum_{i=1}^{k} d_{1}^{i}-\alpha_{1} \sum_{\sum_{i=1}^{k} \gamma^{i}=\gamma} \prod_{i=1}^{k} \\
& \left\{\sup _{0 \leq \theta \leq 1} \Phi_{t}^{J\left(d^{i}-\lambda^{i, j}\right)_{3}-\mu i, j}{ }_{2}^{-\gamma i}{ }_{2}(x, \xi+\theta(\eta-\xi))\right. \\
& \left.\times \sup _{0 \leq \theta \leq 1} \varphi^{J\left(d^{i}-\lambda^{i, j}\right)_{2}-\mu i, j}{ }_{1}^{-\gamma i}{ }_{1}(x, \xi+\theta(\eta-\xi))\right\}
\end{aligned}
$$

where $\Phi_{t}, \varphi$ are functions in Fact 4.8.

We denote by $|\mathscr{F}(\alpha, \beta)|_{\gamma}$ the infimum of constants $C_{\alpha \beta \gamma}$ satisfying (6.1) for $\mathscr{F}=$ $\left\{\mathscr{F}_{\alpha \beta}\right\}$. We denote by $F\left(d^{1}, \cdots, d^{k}\right)$ for $d^{1}, \cdots, d^{k}$ as above the set of all $b(t, x, y$, 
$\xi, \eta) \in C^{\infty}\left(\boldsymbol{R}_{T}^{5}\right)$ such that there exists $\mathscr{I} \in S\left(d^{1}, \cdots, d^{k}\right)$ satisfying with the notations as above

$$
\partial_{t, x, \xi}^{\alpha} \partial_{y, \eta}^{\beta} b(t, x, y, \xi, \eta)=\sum_{j=1}^{n(\alpha, \beta)} b_{j}^{\alpha \beta}(t, x, y, \xi, \eta) \text { for any } \alpha, \beta .
$$

Then we have the followings.

(1) Let $b_{1} \in F\left(d^{1}, \cdots, d^{k}\right), b_{2} \in F\left(e^{1}, \cdots, e^{l}\right)$, and set $b=b_{1} \cdot b_{2}$. Let $\mathscr{F}^{(1)} \in S$ $\left(d^{1}, \cdots, d^{k}\right), \mathscr{F}^{(2)} \in S\left(e^{1}, \cdots, e^{l}\right)$ be families for $b_{1}, b_{2}$ as above respectively. Then $b \in F\left(d^{1}, \cdots, d^{k}, e^{1}, \cdots, e^{l}\right)$ and one can find a family $\mathscr{F}$ for $b$ as above such that

$$
\left|\mathscr{F}^{\prime}(\alpha, \beta)\right|_{\gamma} \leq 2^{|\alpha|+|\beta|+|\gamma|} \sup _{\substack{\alpha^{1}+\alpha^{2}=\alpha \\ \beta^{1}+\beta^{2}=\beta \\ \gamma^{1}+\gamma^{2}=\gamma}}\left|\mathscr{F}^{(1)}\left(\alpha^{1}, \beta^{1}\right)\right|_{\gamma^{1}}\left|\mathscr{F}^{(2)}\left(\alpha^{2}, \beta^{2}\right)\right|_{\gamma^{2}} .
$$

(2) Let $d^{1}, \cdots, d^{k}$ as above be given. Then there exist $C_{\alpha}>0$ and $L_{\alpha} \in \mathbb{N}$ for any $\alpha \in \mathbb{Z}_{+}^{3}$ such that if $b \in F\left(d^{1}, \cdots, d^{k}\right)$, if $\mathscr{F} \in S\left(d^{1}, \cdots, d^{k}\right)$ is a family for $b$ as above, and if

$$
b_{0}(t, x, \xi)=O S-\iint e^{-i(y-x)(\eta-\xi)} b(t, x, y, \xi, \eta) d y d \eta,
$$

we have

$$
\begin{aligned}
& \left|\partial^{\alpha} b_{0}(t, x, \xi)\right| \leq C_{\alpha}\left(\sup _{\substack{\lambda+(0, \mu)=\alpha \\
|\nu| \leq L_{\alpha}}} n(\lambda, \mu)\left|\mathscr{F}_{(\lambda, \mu)}\right|_{\vartheta}\right) t \sum_{i=1}^{k} d_{1}^{i}-\alpha_{1} \\
& \quad \times\left(\Phi_{t} \sum_{i=1}^{k} J\left(d^{i}\right)_{3}+J(-\alpha)_{3}\right)(x, \xi) \cdot\left(\varphi \sum_{i=1}^{k} J\left(d^{i}\right)_{2}+J(-\alpha)_{2}\right)(x, \xi) \\
& \quad \text { for any } \alpha \in \mathbb{Z}_{+}^{3} .
\end{aligned}
$$

Indeed, (1) is a simple concequence of Leibniz rule and (2) follows by noting

$$
\begin{aligned}
\partial^{\alpha} b_{0}(t, x, \xi)= & \sum_{\lambda+(0, \mu)=\alpha}\left(\begin{array}{l}
\alpha \\
\lambda
\end{array}\right) \text { oS }-\iint e^{-i(y-x)(\eta-\xi)} \\
& \times \partial_{i, x, \xi}^{\lambda} \partial_{y, \eta}^{\mu} b(t, x, y, \xi, \eta) d y d \eta,
\end{aligned}
$$

by using (6.2), and by applying Lemma 4.7 with $B$ in Fact 4.8 and $l=k$.

Let us define the topology in $\Sigma^{d}$ by the semi-norms

$$
|a|_{\alpha}^{(d)}=\sup _{t, x, \xi}\left|\partial^{\alpha} a\right| \tilde{\psi}_{1}^{-d_{1}+\alpha_{1}}\left(\langle\xi\rangle^{-1 / 2}\right)^{-d_{2}+\alpha_{2}} \cdot \psi_{3,1 / 2}^{-d_{3}+\alpha_{3}} \text { for } \alpha \in \mathbb{Z}_{+}^{3} .
$$

Then $\Sigma^{d}$ becomes a Frechet space.

Lemma 6.3. Let $a_{1} \in \Sigma^{d}, a_{2} \in \Sigma^{e}$, and $\tau_{i} \in \Sigma^{(0,-2,0)}, i=1,2$ satisfying $\partial_{x} \tau_{i} \in \Sigma^{(0,-2,0)}$ and $\partial_{\xi} \tau_{i} \in \Sigma^{0}$. Set $\pi_{i}=\tau-\tau_{i}(t, x, \xi)$ and for $\theta \in[0,1]$, define

$$
\begin{aligned}
& I_{1, \theta}=I_{1, \theta}^{\pi_{1}}\left[a_{1}, a_{2}\right]=O S-\iint e^{-i y \eta} a_{1}(t, x, \xi+\theta \eta) \pi_{1}(t, x, \tau, \xi+\theta \eta) a_{2}(t, x+y, \xi) \\
& \quad d y d \eta,
\end{aligned}
$$




$$
\begin{aligned}
& I_{2, \theta}=I_{2, \theta}^{\pi_{1}, \pi_{2}}\left[a_{1}, a_{2}\right]=O S-\iint e^{-i y \eta} a_{1}(t, x, \xi+\theta \eta)\left(\pi_{1} \pi_{2}\right)(t, x, \tau, \xi+\theta \eta) \\
& \quad \times a_{2}(t, x+y, \xi) d y d \eta, \\
& I_{3, \theta}=I_{3, \theta}^{\pi_{1}}\left[a_{1}, a_{2}\right]=O S-\iint e^{-i y \eta} a_{1}(t, x, \xi+\theta \eta) \pi_{1}(t, x+y, \tau, \xi) \\
& \quad \times a_{2}(t, x+y, \xi) d y d \eta .
\end{aligned}
$$

Then, for $i=1$ or 3 there exists $b_{j, \theta}(t, x, \xi) \in C^{\infty}\left([0,1]_{\theta} \times \boldsymbol{R}_{T}^{3}\right), j=1,2$ such that $\left\{b_{j, \theta}\right\}_{\theta}$ and $\left\{b_{2, \theta}\right\}_{\theta}$ are bounded in $\Sigma^{d+e}$ and $\Sigma^{d+e-(0,1,0)}$ respectively and satisfying $I_{i, \theta}=b_{1, \theta} \pi_{1}+b_{2, \theta}$, and for $i=2$ there exist $b_{j, \theta}(t, x, \xi) \in C^{\infty}\left([0,1]_{\theta} \times \boldsymbol{R}_{T}^{3}\right), j=1$, $\cdots, 4$ such that $\left\{b_{1, \theta}\right\}_{\theta},\left\{b_{2, \theta}\right\}_{\theta} \cup\left\{b_{3, \theta}\right\}_{\theta}$, and $\left\{b_{4, \theta}\right\}_{\theta}$ are bounded in $\Sigma^{d+e}$, $\sum^{d+e-(0,1,0)}$, and $\sum^{d+e-(0,2,0)}$ respectively and satisfying

$$
I_{2, \theta}=b_{1, \theta} \pi_{1} \pi_{2}+\sum_{j=1}^{2} b_{1+j, \theta} \pi_{j}+b_{4, \theta} .
$$

Proof. Applying Lemma 6.1-(ii) for $i=1,2$ and -(i) for $i=3$ we obtain

$$
\begin{aligned}
I_{1, \theta}= & O S-\iint e^{-i y \eta} a_{1}(t, x, \xi+\theta \eta)\left(\pi_{1}(t, x, \tau, \xi) a_{2}(t, x+y, \xi)\right. \\
& \left.-\tilde{\tau}_{1}(\theta, t, x, \xi, \eta) D_{x} a_{2}(t, x+y, \xi)\right) d y d \eta, \\
I_{2, \theta}= & O S-\iint e^{-i y \eta} a_{1}(t, x, \xi+\theta \eta)\left(\left(\pi_{1} \pi_{2}\right)(t, x, \tau, \xi) a_{2}(t, x+y, \xi)\right. \\
& -\sum_{\{k, j\}=\{1,2\}} \tilde{\tau}_{k}(\theta, t, x, \xi, \eta) \pi_{j}(t, x, \tau, \xi) D_{x} a_{2}(t, x+y, \xi) \\
& \left.+\left(\tilde{\tau}_{1} \tilde{\tau}_{2}\right)(\theta, x, \xi, \eta) D_{x}^{2} a_{2}(t, x+y, \xi)\right) d y d \eta, \\
I_{3, \theta}= & O S-\iint e^{-i y \eta}\left(a_{1}(t, x, \xi+\theta \eta) \pi_{1}(t, x, \tau, \xi)-\theta \partial_{\xi} a_{1}(t, x, \xi+\theta \eta)\right. \\
& \left.\times \tilde{\tilde{\tau}}_{1}(t, x, y, \xi)\right) a_{2}(t, x+y, \xi) d y d \eta,
\end{aligned}
$$

where, by definition, for $a \in \Sigma$,

$$
\tilde{a}(\theta, t, x, \xi, \eta)=\theta \int_{0}^{1} \partial_{\xi} a(t, x, \xi+\theta s \eta) d s, \tilde{\tilde{a}}(t, x, y, \xi)=\int_{0}^{1} \partial_{x} a(t, x+s y, \xi) d s .
$$

Now the desired results follow from Lemma 6.2 in view of the assumption on $a_{i}, \tau_{i}$.

For $q(t, x, \tau, \xi) \in C^{\infty}\left(\boldsymbol{R}_{T}^{4}\right)$ we define ${ }^{f} q \in C^{\infty}\left(\boldsymbol{R}_{T}^{4}\right)$ by

$$
{ }^{f} q(t, x, \tau, \xi)=q(t, x, \tau, f(x) \xi) .
$$

Now, we are ready to prove the following two commutation lemmas.

Lemma 6.4. Let $L_{i}=O p \pi_{i}, i=1,2,3$ with $\pi_{i}=\tau-\tau_{i}, \tau_{i}={ }^{f} \tilde{\tau}_{i}, \tilde{\tau}_{i} \in \sum_{1 / 2}^{(0,-1,1)}$ satisfying with $\varepsilon \in(0,1)$,

$$
\partial_{x} \tilde{\tau}_{i} \in \Sigma_{1 / 2}^{(0,-1,1)}, \partial_{\xi} \tilde{\tau}_{i} \in \Sigma_{1 / 2}^{0}, \partial_{t} \tilde{\tau}_{i} \in t^{\varepsilon-1} \Sigma_{1 / 2}^{(0,-1,1)} .
$$


Then, for $A=O p a, a \in \Sigma$,

(i) $A L_{i}-O p\left(a \pi_{i}\right) \in\left\{\begin{array}{l}\mathcal{A}^{1, N} \text { if } a=O p \tau_{j} \text { for some } j, N \geq 1, \\ \mathcal{A}^{k+1 / 2, l, N} \text { if } A \in \mathcal{A}^{k, l, N} \text { and } N \geq k+1 / 2, \\ \mathcal{B}^{k+1 / 2, l, N} \text { if } A \in \mathscr{B}^{k, l, N} \text { and } N \geq k+1 / 2,\end{array}\right.$

(ii) $L_{i} L_{j}-O p\left(\pi_{i} \pi_{j}\right) \in t^{8-1} \mathscr{A}^{1, N}$ for $N \geq 1$,

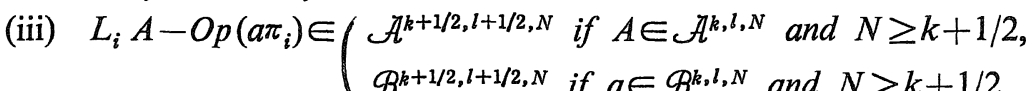

(iv) $A O p\left(\pi_{i} \pi_{j}\right)-O p\left(a \pi_{i} \pi_{j}\right)=A_{i} L_{i}+A_{j} L_{j}+A_{i j}$ with

(1) $A_{i}, A_{j} \in f^{1 / 2} \mathscr{A}^{k+1 / 2, l, N-1 / 2}, A_{i j} \in \mathcal{A}^{k+1, l, N}$ if $A \in \mathcal{A}^{k, l, N}, N \geq k+3 / 2$,

(2) $A_{\imath}, A_{j} \in \mathscr{B}^{k+1 / 2, l, N}, A_{i j} \in \mathscr{B}^{k+1, l, N}$ if $A \in \mathscr{B}^{k, l, N}, N \geq k+1$,

(3) $A_{i}, A_{j} \in f^{1 / 2} \mathscr{A}^{1, N}, A_{i j} \in \mathcal{A}^{3 / 2, N}$ if $A=O p \tau_{l}$ for some $l, N \geq 3 / 2$,

(v) $A L_{i} L_{j}-O p\left(a \pi_{i} \pi_{j}\right)=A_{i} L_{i}+A_{j} L_{j}+A_{i j}$ with

(1) $A_{i}, A_{j} \in f^{1 / 2} \mathcal{A}^{k+1 / 2, l, N-1 / 2}, A_{i j} \in t^{\varepsilon-1} \mathscr{A}^{k+1, l, N}$ if

$A \in \mathcal{A}^{k, l, N}, N \geq k+3 / 2$,

(2) $A_{i}, A_{j} \in \mathscr{B}^{k+1 / 2, l, N}, A_{i j} \in t^{\varepsilon-1} \mathscr{B}^{k+1, l, N}$ if $\mathscr{A} \in \mathscr{B}^{k, l, N}, N \geq k+1$,

(vi) $L_{i} L_{j} L_{k}-O p \pi=\sum_{k=1}^{3} A_{1} L_{1}+A_{i j k}$

where $\pi=\prod_{i=1}^{3} \pi_{i}$, with $A_{l} \in t^{\varepsilon-1} \mathcal{A}^{1,3}$ and $A_{i j k} \in t^{\varepsilon-1} \mathcal{A}^{3 / 2,1 / 2,3}$.

To state the next lemma we make a definition.

Definition 6.5. Let $L_{i}, i=1,2,3$, be three operators as in Lemma 6.4. We define

$\mathcal{C}=\mathcal{C}\left(L_{\imath}\right):=$ the set of all operators on $\mathcal{S}_{T}$ of the form:

$$
\begin{aligned}
& \sum_{i \neq j} A_{i j} L_{i} L_{j}+\sum_{i=1}^{3} A_{i} L_{i} \text { with } A_{i j} \in O p^{f} \Sigma_{1 / 2}^{(-1,0,0)}+f^{1 / 2} \mathcal{A}^{1 / 2,1 / 2,3}, \\
& A_{i} \in O p^{f} \Sigma_{1 / 2}^{(-2,0,0)}+f^{1 / 2} \mathcal{A}^{1,1,3}:
\end{aligned}
$$

$F:=F\left(L_{i}\right)=$ the set of all symbols $q$ with $O p q \in \mathcal{C}\left(L_{i}\right)$;

$E:=$ the set of symbols of the form

$$
\begin{aligned}
& \sum_{k=0}^{2} a_{k} \tau^{k} \text { with } \\
& a_{2} \in t^{-1}\left(\sum^{(0,1,0)}+f^{3 / 2} \Sigma^{(0,1,0)}\right), a_{1} \in t^{-1} B^{1 / 2,1 / 2,3 / 2}, \\
& a_{0} \in \sum_{k=0}^{\min (2, j)} \sum_{l=0}^{3} \sum_{j=0}^{3-l} t^{-(k / 2+3-l-j)} B^{(l+j) / 2, l / 2,3 / 2} ; \\
& \mathcal{C}_{0}:=O p E .
\end{aligned}
$$

Lemma 6.6. Let $L_{i}, \tau_{i}, \tilde{\tau}_{i}, \pi_{\sharp}, i=1,2,3$ be as in Lemma 6.4 and set $\pi=\prod_{i=1}^{3} \pi_{i}$ and let $\varphi \in \Sigma_{1 / 2}^{0}$.

(i) $\pi \circ{ }^{f} \varphi-\pi \cdot{ }^{f} \varphi \in F+E$.

(ii) Let $q(t, x, \tau, \xi)=\sum_{k+l \leq 3} a_{k l}(t, x) \xi^{l} \tau^{k}, a_{k l} \in B_{h, T}, a_{30}=1$. Assume that $\pi$ 
$(t, x, \tau, \xi)={ }^{f} q(t, x, \tau, \xi)$ when $(t, x, \xi) \in \operatorname{supp}{ }^{f} \varphi$. Then it follows that $\pi^{f} \varphi-{ }^{f} \varphi \circ{ }^{f} q \in F+E$.

Before the proof of these lemmas we note

Fact 6.7. (1) If $a \in \Sigma_{1 / 2}^{d}$ (resp. $\Sigma^{d}$ ) with $\mu_{1 / 2}(d) \geq 0$ and $d_{3} \geq 0$ (resp. $\mu(d) \geq 0$ and $\nu(d) \geq 0$ ), $\partial_{\xi}^{j} f a \in f^{j / 2} \Sigma^{J_{1 / 2}(d)+(0, j, 0)}$ (resp. $\partial_{\xi}^{j f} a \in f^{j / 2} \Sigma^{d+(0, j, 0)}$ ). This follows from Fact 4.6-4 and $-7,(4.3)$, and (4.6). (See the proof of Corollary $4.12-$ (i).)

(2) Let $a \in A^{k, l, N}$ (resp. $B^{k, l, N}$ ). Then $\partial_{\xi}{ }^{j}$ a is a sum of the symbols of the form: $a_{1}+f^{N} a_{2} ; a_{1} \in f^{j / 2} \Sigma^{J_{1 / 2}(d)+(0, j, 0)} \cap f^{j f} \sum_{1 / 2}^{d-(0,0, j)}$ (resp. $f^{j / 2} \Sigma^{d+(0, j, 0)} \cap f^{j f}$ $\left.\Sigma^{d-(0,0, j)}\right), a_{2} \in \sum^{e+(0, j, 0)}$ with $d \in \Delta^{k, l}$ (resp. $\left.\Delta_{k, l}\right), e \in \Delta_{k, l}$.

(3) Let $\tau_{\imath}$ be symbols in Lemma 6.4. Then from the proof of Corollary 4. 12-(i),

$$
\partial_{\xi}^{j} \tau_{i} \in f^{(j+1) / 2} \Sigma^{(0, j-1,0)} \cap f^{j f} \Sigma_{1 / 2}^{(1-j)(0,0,1)} \cap f^{j f} \Sigma^{(1-j)(0,0,1)}
$$

for $j \neq 0 . \quad \tau_{i}$ as well as $\tilde{\tau}_{i}$ also satisfies (6.4), and

$$
f^{\theta} \partial_{x}^{j} \tau_{i} \in{ }^{f} \Sigma_{1 ; 2}^{(0,-j, 1)} \cap^{f} \Sigma^{(0,-j-1,0)}
$$

for $j \neq 0, \theta>0$. This follows from (4.16).

Proof of Lemma 6.4. (i) follows from Lemma 4.18-(3)-(i) and -(iv). (ii) follows from (i) and the assumption on $\tau_{i}$. (iii) follows from Lemmas 4.18(3)-(i) and 4.22. Let us show (1) of (iv). We have

$$
\begin{aligned}
& a \circ\left(\pi_{i} \pi_{j}\right)-a \pi_{i} \pi_{j}=\sum_{1=1}^{M-1} l !^{-1} \partial_{\xi}^{l} a(t, x, \xi) D_{x}^{l}\left(\pi_{i} \pi_{j}\right)(t . x . \tau, \xi) \\
& \quad+(2 \pi)^{-1}(M-1) !^{-1} \int_{0}^{1}(1-\theta)^{M-1}\left(O S-\iint e^{-i y^{\eta}} \partial_{\xi}^{M} a(t, x, \xi+\theta \eta)\right. \\
& \left.\quad \times D_{x}^{M}\left(\pi_{i} \pi_{j}\right)(t, x+y, \tau, \xi) d y d \eta\right) d \theta .
\end{aligned}
$$

From Fact 6.7-(2) and -(3) the summation in (6.5) takes the form:

$$
b_{i} \pi_{i}+b_{j} \pi_{j}+b_{i j} \text { with } b_{i}, b_{j} \in f^{1 / 2} \mathcal{A}^{k+1 / 2, l, N-1 / 2}, b_{i j} \in \mathcal{A}^{k+1, l, N} .
$$

Since $b_{i}=f^{1 / 2} c_{i}$ for some $c_{i} \in \mathcal{A}^{k+1 / 2, l, N-1 / 2}$, the summation defines the operator in the desired form by (i). The oscillatory integrals in the integral terms are sums of terms with the notation in Lemma 6.3:

$$
r_{\theta}\left[\partial_{\xi}^{M} a, D_{x}^{l} \tau_{i} D_{x}^{M-l} \tau_{j}\right], 1 \leq l \leq M ; I_{3, \theta}^{\pi_{k}}\left[\partial_{\xi}^{M} a, D_{x}^{M} \tau_{l}\right],\{k, l\}=\{i, j\} .
$$

Thus from Lemma 4.9 and 6.3, the integral term takes the form (6.6) when $M \geq 2 N$ so that this term defines the operator in the desired form (i). (2) and (3) can be shown in the similar way. 
In view of the equality

$$
A L_{i} L_{j}-O p\left(a \pi_{i} \pi_{j}\right)=A\left(L_{i} L_{j}-O p\left(\pi_{i} \pi_{j}\right)\right)+O p\left(a \circ\left(\pi_{i} \pi_{j}-a \pi_{i} \pi_{j}\right)\right),
$$

(v) follows immediately from (ii), (iv), Lemma 4.18-(1), and the fact that $A^{k, l, N} \subset B^{k, l, N}$ in general.

To see (iv) we note that

$$
\begin{gathered}
L_{i} L_{j} L_{k}-O p \pi=\left(L_{j} L_{k}-O p\left(\pi_{j} \pi_{k}\right)\right) L_{i}+\left[L_{i}, L_{j} L_{k}-O p\left(\pi_{j} \pi_{k}\right)\right] \\
+\left[D_{t}, O p\left(\pi_{j} \pi_{k}\right)\right]-\left(O p \tau_{i} \cdot O p\left(\pi_{j} \pi_{k}\right)-O p\left(\tau_{i} \pi_{j} \pi_{k}\right)\right) .
\end{gathered}
$$

The first and last terms have the desired forms respectively from (ii) and (iv). From (i) and (ii) we see

$$
\left[L_{i}, A\right] \in \mathcal{A}^{k+1 / 2, l+1 / 2, N} \text { if } A \in \mathcal{A}^{k, l, N} \text { and } N \geq k+1 / 2 .
$$

So the second term has the desired form in view of (ii) and that in general

$$
t^{-1} \mathcal{A}^{k, l, N} \subset \mathcal{A}^{k+1 / 2, l+1 / 2, N} \text { if } N \geq k+1 / 2 \text {. }
$$

Finally, the symbol of the third term equals to $-D_{t} \tau_{j} \cdot \pi_{k}-D_{t} \tau_{k} \cdot \pi_{j}$. Hence, one can easily see, from the fact that $\partial_{t} \tau_{i} \in t^{\varepsilon-1} \mathscr{A}^{1, N}$ for $N \geq 1$ and (i), that this term the desired form. This completes the proof.

Remark 6.8. The presence of the factor $f^{1 / 2}$ for $A_{i}$ in (v)-(i) in Lemma 6.4 is important.

Proof of Lemma 6.6. (i) We have

$$
\begin{aligned}
& \pi \circ^{f} \varphi=\sum_{s=0}^{3} s !^{-1} \sum_{j=0}^{N-1} j !^{-1} \partial_{\tau}^{s} \partial_{\xi}^{j} \pi \cdot D_{t}^{s} D_{x}^{j} f \varphi(t, x, \tau, \xi) \\
& +\sum_{s=0}^{2} s !^{-1}(N-1) !^{-1}(2 \pi)^{-1} \int_{0}^{1}(1-\theta)^{N-1}\left(O S-\iint e^{-i y \eta} \partial_{\tau}^{s} \partial_{\xi}^{N} \pi(t, x, \tau, \xi+\theta \eta)\right. \\
& \left.\times D_{z}^{s} D_{x}^{N} \varphi(t, x+y, \xi) d y d \eta\right) d \theta .
\end{aligned}
$$

Since $f^{\lambda} D_{t}^{s} D_{x}^{j f} \varphi \in{ }^{f} \Sigma_{1 / 2}^{(-s,-j, 0)}$ for any $\lambda>0$ from Fact $4.6-2$, in view of Fact $6.7-(3)$ the first summation minus $\pi \cdot f \varphi$ takes the form

$$
\sum_{k \neq 1} b_{k l} \pi_{k} \pi_{l}+\sum_{k=1}^{3} b_{k} \pi_{k}+b_{0}
$$

with $b_{k l} \in f \Sigma_{1 / 2}^{(-1,0,0)}+f^{1 / 2} \bigcap_{N \geq 3 / 2} A^{1 / 2, N}, b_{k} \in{ }^{f} \Sigma_{1 / 2}^{(-2,0,0)}+f^{1 / 2} \bigcap_{N \geq 3 / 2} A^{1,1 / 2, N}$ for $k \neq 0$, $b_{0} \in \Sigma^{f(-3,0,0)}+f^{1 / 2} \bigcap_{N \geq 3 / 2} A^{3 / 2,1, N}$.

The symbols of the form (6.10) are in $F+E$ in view of Lemma 6.4-(i) and $-(\mathrm{v})$. On the other hand, the oscillatory integrals in the integral terms are 
sums of terms with notations in Lemma 6.3 respectively in $s$ as in the following.

$$
\begin{aligned}
& s=0: I_{1, \theta}^{\pi}{ }_{p}\left[\partial_{\xi}^{N-k} \tau_{q} \partial_{\xi}^{k} \tau_{r}, D_{x}^{N}{ }^{f} \varphi\right],\{p, q, r\}=\{1,2,3\}, 0<k<N ; \\
& I_{2, \dot{p}}^{\pi_{p}, \pi_{q}}\left[\partial_{\xi}^{N} \tau_{r}, D_{x}^{N} f_{\varphi}\right],\{p, q, r\}=\{1,2,3\} ; \\
& r_{\theta}\left[\prod_{i=1}^{3} \partial_{\xi}^{j_{i}} \tau_{i}, D_{x}^{N} f_{\varphi}\right], \sum_{i=1}^{3} j_{i}=N, j_{i}>0 \text {. } \\
& s=1: I_{1, \theta}^{\pi}\left[\partial_{\xi}^{N} \tau_{q}, D_{t} D_{x}^{N}{ }^{f} \varphi\right], p \neq q ; r_{\theta}\left[\partial_{\xi}^{N-k} \tau_{p} \partial_{\xi}^{k} \tau_{q}, D_{t} D_{x}^{N} f_{\varphi}\right], 0<k<N \text {. } \\
& s=2: r_{\theta}\left[\partial_{\xi}^{N} \tau_{p}, D_{t}^{2} D_{x}^{N} f_{\varphi}\right] \text {. }
\end{aligned}
$$

Thus, by Lemmas 6.3 and 4.9 the summation of the integral terms takes the form

$$
\begin{aligned}
& \sum_{k \neq 1} c_{k l} \pi_{k} \pi_{l}+\sum_{k=1}^{3} c_{k} \pi_{k}+c_{0} \\
& \quad \text { with } c_{k l} \in f^{(N+1) / 2} \Sigma^{(0,-1,0)}, c_{k} \in f^{(N+1) / 2} \Sigma^{(-1,-1,0)} \text { for } k \neq 0, \\
& \quad c_{0} \in f^{(N+1) / 2} \Sigma^{(-2,-1,0)} .
\end{aligned}
$$

From Lemma 6.4 -(i) and (v) the symbols of the form (6.11) are in $F+E$ when $N \geq 6$. This proves (i).

(ii) We have that

$$
\begin{aligned}
& { }^{f} \varphi \circ{ }^{f} q-{ }^{f}(\varphi q)=\sum_{j=1}^{N-1} j !^{-1} \partial_{\xi}^{j} f \varphi \cdot D_{x}^{j} f q \\
& +(N-1) !^{-1} \sum_{k<3} r_{N}\left[\partial_{\xi}^{N} f \varphi, a_{k l}(f \xi)^{l}\right] \tau^{k} .
\end{aligned}
$$

Since $\partial_{\xi}^{N} f_{\varphi} \in f^{N / 2} \Sigma^{(0, N, 0)}$, the second summation $\in E$ for $N \geq 6$ in view of the definition of $E$. On the other hand ,from the assumption, for $j \neq 0$.

$$
\partial_{\xi}^{j} f^{\prime} \varphi \cdot \partial_{x}^{j} f^{\prime} q=\sum_{\substack{I \subset\{1,2,3\} \\
0<\notin(I) \leq 3}} \prod_{i \in I^{c}} \pi_{i} \cdot\left(\sum_{\substack { J=\left(j_{j}\right) \in N^{\sharp}(I) \\
\begin{subarray}{c}{\sum_{k} j_{k}=i{ J = ( j _ { j } ) \in N ^ { \sharp } ( I ) \\
\begin{subarray} { c } { \sum _ { k } j _ { k } = i } }\end{subarray}} C_{I, J} \partial_{\xi}^{j} f^{\prime} \varphi \cdot \prod_{k \in I} \partial_{x}^{j k} \tau_{k}\right)
$$

where $\prod_{i \in I^{c}} \pi_{i}$ means 1 when $I^{c}=\phi$.

Since $\partial_{\xi}^{j} f \varphi \cdot \prod_{k \in I} \partial_{x}^{j} \tau_{k} \in f^{j-1 / 2 f} \Sigma^{(0,-\sharp,(I), 0)}$, this is the symbol of the from (6.10) so that it is in $F+E$ from the proof of (i). This completes the proof.

Lemma 6.9. Let $\tau_{i} \in{ }^{f} \Sigma_{0}^{(0,0,2)}, i=1,2,3$ and set $\pi=\prod_{i=1}^{3}\left(\tau-\tau_{i}\right)$. Let $\varphi \in \Sigma^{0}$ and let $q(t, x, \tau, \xi)=\sum_{k+l \leq 3} a_{k l}(t, x) \xi^{l} \tau^{k}, a_{k l} \in B_{h, T}$. Assume that $\pi(t, x, \tau, \xi)$ $={ }^{f} q(t, x, \tau, \xi)$ when $(t, x, \xi) \in \operatorname{supp}{ }^{f} \varphi$. Then,

$$
\pi \circ f \varphi-{ }^{f} q \circ f \varphi \in E .
$$

Proof. We have 


$$
\begin{aligned}
& \pi \circ{ }^{f} \varphi=\sum_{j+k \leq 3}(j ! k !)^{-1} D_{\tau}^{j} D_{\xi}^{k} \pi \cdot \partial_{t}^{j} \partial_{x}^{k}{ }^{f} \varphi+\sum_{j+k=4}(j !(k-1) !)^{-1} \\
& \quad \times r_{k}\left[D_{\tau}^{j} D_{\xi}^{k} \pi, \partial_{t}{ }^{j} \partial_{x}{ }^{k}{ }^{f} \varphi\right] .
\end{aligned}
$$

From (4.3) and (4.6), it follows that if $\tau_{j}={ }^{f} \tilde{\tau}_{j}$ with $\tilde{\tau}_{j} \in \Sigma_{0}^{(0,0,2)}$,

$$
\begin{aligned}
& \partial_{\xi}^{k} \tau_{j}=f^{k f} \partial_{\xi}^{k} \tilde{\tau}_{j}=f^{\lambda}(f\langle\xi\rangle\langle f \xi\rangle)^{k-\lambda} \\
& \quad \times^{f}\left(\langle\xi\rangle^{k-\lambda} \partial_{\tilde{\xi}}^{k} \tilde{\tau}_{j}\right)\langle\xi\rangle^{-k+\lambda} \in f^{\lambda} \Sigma^{(k-\lambda)(0,2,0)}
\end{aligned}
$$

for $k \in N, \lambda \in \boldsymbol{R}, 1 \leq \lambda<k$. This implies particularly

$$
\begin{aligned}
& \partial_{\xi}^{4} \tau_{j} \in f^{3 / 2} \Sigma^{5(0,1,0)}, \partial_{\xi}^{3} \tau_{j} \in f \Sigma^{4(0,1,0)} \cap f^{3 / 2} \Sigma^{3(0,1,0)}, \\
& \partial_{\xi}^{2} \tau_{j} \in f^{3 / 2} \Sigma^{(0,1,0)} \cap f \Sigma^{2(0,1,0)} .
\end{aligned}
$$

Using this and Lemma 4.3, one can easily see that the second summaiton in (6.12) takes the form

$$
f^{3 / 2}\left(b_{2} \tau^{2}+b_{1} \tau+b_{0}\right) \text { with } b_{2} \in \Sigma^{(0,1,0)}, b_{1} \in \Sigma^{(-1,0,0)}, b_{0} \in \Sigma^{(-2,-1,0)},
$$

which is in $F+E$. On the other hand, from the assumption

$$
D_{\tau}^{j} D_{\xi}^{k} \pi \cdot \partial_{\xi}^{j} \partial_{x}^{k} f \varphi=D_{\tau}^{j} D_{\xi}^{k} q \cdot \partial_{t}^{j} \partial_{x}^{k} f_{\varphi}
$$

so that the first summation is equals to ${ }^{f} q \circ{ }^{f} \varphi$. This completes the proof.

Lemma 6.10. Let $L_{i}=D_{t}-a_{i}(t, x) f(x) D_{x}, a_{i} \in B_{h, T}, i=1,2,3$.

(1) $L_{1} L_{2}=O p\left(\prod_{i=1}^{2}\left(\tau-a_{i} f \xi\right)\right)+t^{l / h-1}$ bf $D_{x}$ for some $b \in B_{h, T}$.

(2) Assume that $a_{1}-a_{2} \in E_{h, T} \backslash\{0\}$. Then there exists $r \in \mathbb{N}$ such that any differential operator of the form $L=b_{1} f D_{x}+b_{2} D_{t}, b_{i} \in t^{r} f^{r} B_{h, T}$ can be expressed as $L=c_{1} L_{1}+c_{2} L_{2}, c_{i} \in B_{h, T}$.

(3) Assume that $a_{i}-a_{j} \in E_{h, T} \backslash\{0\}$ for any distinct $i, j$. Then there exists $r \in \mathbb{N}$ such that any differential operator of the form $R=\sum_{0<i+j \leq 2} b_{i j} f^{i} D_{x}^{i} D_{t}^{j}, b_{i j} \in t^{r} f^{r} B_{h, T}$ can be expressed as $R=\sum_{i \neq j} c_{i j} L_{i} L_{j}+\sum_{i} c_{i} L_{i}, c_{i j}, c_{i} \in B_{h, T}$.

(4) Set $Q=O p\left(\prod_{i=1}^{3}\left(\tau-a_{i} f \xi\right)\right)$. Then for any $\alpha \geq 0,\left[Q, f^{\alpha}\right]$ is expressed as $\sum_{i \neq j} b_{i j} L_{i} L_{j}+\sum_{i} b_{i} L_{i}+t^{1 / h-1} c_{1} f D_{x}+c_{0}$ with $b_{i j}, b_{i}, c_{i} \in B_{h, T}$.

(5) Let $R=\sum_{i+j \leq m} a_{i j} f^{i} D_{x}^{i} D_{t}^{j}, a_{i j} \in B_{h, T}, m \geq 1$, and let $a \in B_{T}$. Then, [R, $\left.a\right]$ can be written in the form $\sum_{i+j \leq m-1} b_{i j} f^{i} D_{x}^{i} D_{t}^{j}, b_{i j} \in B_{h, T}$.

(1) is trivial, (2) and (3) is a simple consequence of Lagrange's interpolation formula and (1), and (4) is that of Leibniz rule and (1). (5) is proved by a direct calculation. The details are omitted. 
Lemma 6.11. Let $L_{j}=D_{t}-A_{j}, L_{j}^{\prime}=D_{t}-A_{j}^{\prime}$, with $A_{j}$ and $A_{j}^{\prime}$ in $\Sigma^{(0,-2,0)}$, $j=1,2,3$, and let $R=D_{t}^{3}+\sum_{j=0}^{2} B_{j} D_{t}^{j}, B_{j} \in O p \Sigma^{-2(0,3-j, 0)}$. Then if $B \in O p \Sigma^{(0,6,0)}$, $L_{1} L_{2} L_{3} B-B R$ can be expressed as $\sum_{i \neq j} B_{i j}^{\prime} L_{i}^{\prime} L_{j}^{\prime}+\sum_{i} B_{i}^{\prime} L_{i}^{\prime}+B_{0}^{\prime}$ with $B_{i j}^{\prime} \in$ $t^{-1} O p \Sigma^{0}, B_{i}^{\prime} \in t^{-2}$ Op $\Sigma^{0}$ for $i \neq 0, B_{0}^{\prime} \in t^{-3}$ op $\Sigma^{0}$.

Lemma 6.11 is proved in a straightforward way by using Lemma 4.13 and the fact that $[L, A] \in O p \Sigma^{(-1,-1,0)+\infty}$ if $L=D_{t}-A_{1}$ with $A_{1} \in O p \Sigma^{(0,-2,0)}$, and if $A \in O p \Sigma^{\alpha}$.

Lemma 6.12. Let $\varphi \in{ }^{f} \Sigma^{0}$ and $q=\sum_{i+j \leq 2} a_{i j} f^{j} \tau^{i} \xi^{j}, a_{i j} \in B_{h, T}$. Then

$$
q \circ \varphi-q \cdot \varphi, \varphi \circ q-q \cdot \varphi \in E .
$$

Proof. Set $b_{i}=\sum_{j \leq 2-i} a_{i j} \xi^{j}$ so that $q=\sum_{i \leq 2}{ }^{f} b_{i} \tau^{i}$. Since $b_{i}$ satisfies (4.41) in Lemma 4.18-(2), we have $\varphi \circ{ }^{f} b_{i}-\varphi \cdot{ }^{f} b_{i},{ }^{f} b_{i} \circ \varphi-\varphi^{f} b_{i} \in B^{3 / 2-i, N}$ for $i<2$ and any $N$. $b_{2}=a_{20}$ and $\varphi \circ a_{20}-\varphi \cdot a_{20} \in{ }^{f} \Sigma^{(0,0,-1)}+f^{3 / 2} \Sigma^{(0,1,0)}$, since $\partial_{\xi}^{k} \varphi \in f^{k / 2} \sum^{k(0,1,0)} \cap$ $f^{k f} \Sigma^{(0,0,-k)}$. Thus, we have proved the second statement and first one is also clear, since moreover, ${ }^{f} b_{i} \circ\left(\tau^{i} \circ \varphi-\tau^{i} \varphi\right) \in E$ for $i \neq 0$ in view of Lemma 4.18-(1).

Lemma 6.13. Let $\varphi \in{ }^{f} \Sigma^{0}$ and $q \in E$. Then $q \circ \varphi, \varphi \circ q \in E$.

Proof. Let $q=\sum_{i \leq 2} a_{i} \tau^{i} \in E$. By virtue of the fact that ${ }^{f} \Sigma^{(0,1-k, 0)} \subset \Sigma^{(0,1-k, 0)}$, $k \in \boldsymbol{N}$, one can show in the same way as in the proof of Corollary 4.12-(i) that

$$
\varphi \circ a_{2}, a_{2} \circ \varphi \in t^{-1}\left({ }^{f} \Sigma^{(0,1,0)}+f^{3 / 2} \Sigma^{(0,1,0)}\right) .
$$

It follows from Lemma 4.18-(1) that $\sum_{i \leq 1} \varphi \circ a_{i} \cdot \tau^{i}, \sum_{i \leq 1} a_{i} \circ \varphi \cdot \tau^{i} \in E$, and also $a_{i} \circ$ $\left(\tau^{i} \circ \varphi-\tau^{i} \varphi\right) \in E$ for $i \neq 0$ by Leibniz rule. These facts prove the result.

Definition 6.14. Let $\varphi \in \Sigma_{1 / 2}^{0}(T, h)$. Then we define the set $\sum_{\varphi}^{d}, d \in \boldsymbol{R}^{3}$ with $d_{1} \leq 0$ by

$$
\sum_{\varphi}^{d}:=\left\{{ }^{f} a ; a \in \sum_{1 / 2}^{d}, \operatorname{supp} a \subset \operatorname{supp} \varphi\right\} .
$$

Definition 6.15. Let $\varphi \in \Sigma_{1 / 2}^{0}$ and $q=\sum_{i+j \leq 3} a_{i j} \tau^{i} \xi^{j}$ with $a_{i j} \in B_{h, T}$ and $a_{30}=1$ be given. We define

$\Delta_{\alpha, i}=\left\{d \in \boldsymbol{R}^{3} ; d_{1}=-\alpha_{1}, \sigma_{1 / 2}(d)=\alpha_{1}+\alpha_{2}-i / 2, \mu_{1 / 2}(d) \geq 0\right\} \quad$ for

$\alpha \in \boldsymbol{Z}_{+}^{3}, i \in \boldsymbol{Z}_{+} ; F_{i}=$ the set of all finite sums of symbols in

$\left\{f^{\left(i-\alpha_{1}\right) / 2} a^{f} \tilde{\partial}^{\alpha} q ; a \in \sum_{\varphi}^{d}, d \in \Delta_{\alpha, i}, 0<|\alpha| \leq i\right\}$ for

$i \in \boldsymbol{N}$, where $\tilde{\partial}^{\alpha}=\partial_{\tau}^{\alpha} \partial_{\xi}^{\alpha} \partial_{x}^{\alpha}$. 
We also define

$$
\begin{aligned}
& q_{\alpha, i}=f^{\left(i-\alpha_{1}\right) / 2} \psi^{d_{\alpha i} i f \tilde{\partial}^{\alpha} q} \text { for } i \in N \text { and } \alpha \in \mathbb{Z}_{+}^{3} \text { with } 0<|\alpha| \leq i, \\
& q_{0,0}={ }^{f} q \text {, and } \\
& \widetilde{F}=\left\{q_{\alpha, i} ; 0<|\alpha| \leq i\right\} \text { for } i \in N \text {, where } \\
& d_{\alpha, i}=\left(-\alpha_{1}, 0,0\right)+\left(\alpha_{1} / 2+\alpha_{2}-i / 2\right)(0,-1,1) \text { for } \alpha \in \mathbb{Z}_{+}^{3} \text { and } i \in \mathbb{Z}_{+}, \\
& \psi^{d}={ }^{d} \tilde{\psi}^{d}, \tilde{\psi}^{d}=\psi_{1}^{d_{1}} \psi_{2}^{d} \psi_{3,1 / 2}^{d_{3}} \text { for } d \in R^{3} .
\end{aligned}
$$

Fact 6.16. (1) $f^{\varepsilon} \partial^{a} f b \in{ }^{f} \sum_{1 / 2}^{d-a}$ for any $b \in \sum_{1 / 2}^{d}$ and $\varepsilon>0$.

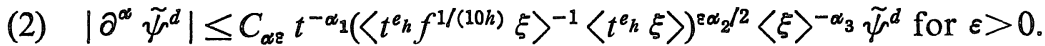

(3) $f^{\varepsilon \alpha_{2}} \partial^{\alpha} \psi^{d} \in t^{-\alpha_{1} f} \sum_{1 / 2}^{d-\alpha_{3}(0,-1,1)}$ for $\varepsilon>0$.

(4) If $q=a \tau^{i} \xi^{j}$ with $a \in B_{h, T}, i+j \leq 3$, and $a=1$ when $i=3$ and $j=0$, we have $q_{\alpha, i}=f^{\left(i-\alpha_{1}\right) / 2 f}\left(b \psi_{1}^{-\alpha_{1}}\langle\xi\rangle^{j+\alpha_{1} / 2-i / 2}\right) \tau^{b}$

with $\alpha_{b}+j+l \leq 3, j \in \mathbb{Z}_{+}, l \leq 2$, and $b \in \Sigma_{0}^{0}$ satisfying $\left|\partial^{\alpha} b\right| \leq C_{\alpha} t^{-\alpha}\langle\xi\rangle^{-\alpha_{3}}$.

Proof. (1) Nothing but Fact 4.6-2. (2) A result of the estimates that

$$
\left|\partial^{\infty}\left(t^{s} f^{s^{\prime}} \xi\right)\right| \leq C_{\alpha}\left\langle t^{s} f^{s^{\prime}} \xi\right\rangle\left(\left\langle t^{s} f^{s^{\prime}} \xi\right\rangle^{-1}\left\langle t^{s} \xi\right\rangle\right)^{\varepsilon{ }_{2}}\langle\xi\rangle^{-\alpha_{3}} \cdot t^{-\alpha_{1}}
$$

for $\varepsilon>0, s \geq 0, s^{\prime} \geq 0$, and Lemma 3.8-3.

(3) A simple consequence of (4.16) and (2).

(4) This is shown by a direct calculation.

Lemma 6.17. (1) Let $q=a \tau^{k}$ with $a \in \in^{f} \Sigma^{d}+f^{3 / 2} \Sigma^{d}, \sigma(d)+k \leq 3 / 2,-d_{1}+$ $k \leq 3, k \leq 2, \tilde{\mu}(d)=\mu\left(\left(0, d_{2}, d_{3}\right)\right) \geq 0$. Then, $q \in E$.

(2) $F_{j} \subset E$ if $j \geq 3$.

(3) $d_{\alpha, i} \in \Delta_{\alpha, i}, \sum_{1 / 2}^{d} \subset \Sigma_{1 / 2}^{d \alpha, i}$ for $d \in \Delta_{\alpha, i}$.

(4) $a \tilde{\psi}^{-d} \in \Sigma_{1 / 2}^{0}$ if $a \in \Sigma_{1 / 2}^{d}$.

(5) $a q_{\alpha, i} \in F_{i}$ for $a \in \Sigma_{\varphi}^{0}$ and $i \in \mathbb{N}$.

(6) any $q \in F_{i}$ can be expressed in the form of a sum of symbols of the form $a q_{\alpha_{0} i}$ with $a \in \Sigma_{\varphi}^{0}$ and $0<|\alpha| \leq i$.

Proof. (1) follows from the inequality that

$$
\tilde{\psi}_{1}^{d}\langle\xi\rangle^{-d_{2} / 2} \psi_{3,1 / 2}^{d_{3}} \leq \tilde{\psi}_{1}^{-(3-k)}\langle\xi\rangle^{-(3-k) / 2+\sigma(d)} .
$$

(2) follows from Fact 4.16-(4) and (1). (3) is clear from the definition. (4) follows from Fact 4.16-(2). (5) follows from (3) and the definition. (6) follows from (3), (4), and the definition of $F_{i}$.

Lemma 6.18. Let $\varphi$ and $q$ as in Definition 6.15 be given and suppose that $i \leq 2$ and $a \in \Sigma_{\varphi}^{0}$. Then, 


$$
a \circ q_{\alpha, i}-a \cdot q_{\alpha, i}, q_{\alpha, i} \circ a-a \cdot q_{\alpha, i} \in \sum_{i<j \leq 2} F_{j}+E .
$$

Proof. In view of Fact 6.16-(4) and -(2) for $i \in \boldsymbol{N}$ one can express $q_{\alpha, i}$ as $\sum_{1=0}^{2} f^{\left(i-\alpha_{1}\right) / 2} f_{l} b^{l}$ where $b_{l}=0$ if $\alpha_{1}+l>3$ and where $b_{l}$

satisfies $\left|\partial^{\beta} b_{l}\right| \leq C_{\beta \varepsilon} \psi_{1}^{-\alpha_{1}}\langle\xi\rangle^{3-l-\alpha_{1} / 2-i / 2-\beta_{3}+\varepsilon \beta_{2}} \cdot t^{-\beta_{1}}$ for $\varepsilon>0$. Thus we have in view of Corollary 4.12-(i) and Lemma 6.17-(1),

$$
a \circ q_{\alpha, i}-\sum_{k=0}^{N} D_{\xi}^{k} a \cdot \partial_{x}^{k} q_{\alpha, i} / k !, q_{\alpha, i} \circ a-\sum_{s=0}^{3} \sum_{k=0}^{N}(s ! k !)^{-1} D_{\tau}^{s} D_{\xi}^{k} q_{\alpha, i} \cdot \partial_{t}^{s} \partial_{x}^{k} a \in E
$$

for sufficiently large $N$ (in fact $N \geq 5$ ). So, in view of Lemma 6.17-(2): it suffices to show that

$$
D_{\xi}^{s} a \cdot \partial_{x}^{s} q_{\alpha, i} \in F_{i+s}, D_{\tau}^{k} D_{\xi}^{s} q_{\alpha, i} \cdot \partial_{t}^{k} \partial_{x}^{s} a \in F_{i+s+k} \text { for } s \in \boldsymbol{N} .
$$

To do so we note that $a={ }^{f} \tilde{a}$ with some $\tilde{a} \in \Sigma_{1 / 2}^{0}$ satisfying supp $\tilde{a} \subset \operatorname{supp} \varphi$, from the assumption. Then

$$
\begin{aligned}
D_{\xi}^{s} a \cdot \partial_{x}^{s} q_{\alpha, i}= & \sum_{\substack{k+|\beta| \leq s \\
\beta_{1}=0}} e_{k, \beta}^{\alpha, i}(x){ }^{f} D_{\xi}^{s} \tilde{a} \cdot f^{\left(i+s-\alpha_{1}\right) / 2} f^{k / 4} \cdot \partial_{x}^{k} \psi^{d \alpha, i} \\
& \times(f \xi)^{\beta_{2} f} \tilde{\partial}^{\alpha+\beta} q,
\end{aligned}
$$

where $e_{k, \beta}^{\alpha, i} \in C_{0}^{\infty}(\boldsymbol{R})$ with $e_{k, 0}^{\alpha, i}=0$ if $|\alpha|=i=0$, and

$$
\begin{aligned}
D_{\tau}^{k} D_{\xi}^{s} q_{\alpha, i} \cdot \partial_{t}^{k} \partial_{x}^{s} a= & \sum_{\substack{\beta=\left(k, \beta_{2}, 0\right) \\
\beta_{2} \leq s}}\left(\begin{array}{c}
s \\
\beta_{2}
\end{array}\right) f^{\left(i+s-\alpha_{1}\right) / 2+s / 2} \\
& \times \partial_{t}^{k} \partial_{x}^{s} a^{f} \partial_{\xi}^{s-\beta_{2}} \tilde{\psi}^{d_{\alpha, i} f} \partial^{\alpha+\beta} q .
\end{aligned}
$$

By Fact $6.16-(3),{ }^{f} D_{\xi}^{s} \tilde{a} f^{k / 4} \partial_{x}^{k} \psi^{d \alpha, i}(f \xi)^{\beta_{2}} \in \sum_{\varphi}^{d \alpha+\beta, i+s}$ so that $D_{\xi}^{s} a \cdot \partial_{x}^{s} q_{\alpha, i} \in F_{i+s}$, for when $|\alpha|=i=0, \beta \neq 0$ for non trivial terms in the summation of (6.14).

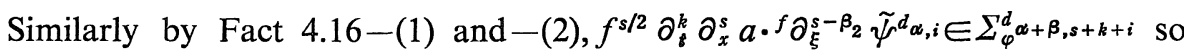
that in view of the fact that when $|\alpha|=i=0, \beta_{2}<s$ implies that $\partial_{\xi}^{s-\beta_{2}} \widetilde{\psi}^{d \alpha, i=0}$ in the summation of (6.15), we obtain the latter of (6.13). This completes the proof.

Lemma 6.19. Let $\varphi$ and $q$ as in Definition 6.15 be given. Set

$$
\Delta=\left\{(\alpha, i) \in \boldsymbol{Z}^{3} \times \boldsymbol{Z}_{+} ; \alpha=0 \text { and } i=0 \text {, or } 0<|\alpha| \leq i \leq 2\right\}, \Delta^{\prime}=\Delta \backslash\{(0,0)\},
$$
and $Q_{\lambda}=O p q_{\alpha, i}$ for $\lambda=(\alpha, i) \in \Delta$. Then for any $s \in \boldsymbol{N}$ there exists $l \in N$ such that for any $\mu \in \Delta$ and $A_{l}, \cdots, A_{s} \in O p \sum_{\varphi}^{0}$ one can find $A_{\lambda j k}, \lambda \in \Delta^{\prime}, j=l, \cdots, l_{s}$, $k=l, \cdots, s, \in O p \Sigma_{\varphi}^{0}$ such that

$$
\left[Q_{\mu}, A_{1} \cdots A_{s}\right]-\sum_{\lambda, j} Q_{\lambda} A_{\lambda j 1} \cdots A_{\lambda j s} \in \mathcal{C}_{0} .
$$


Proof. Set $q_{\lambda}=q_{\alpha, i}$ for $\lambda=(\alpha, i) \in \Delta$. Let $a \in \Sigma_{\varphi}^{0}$ and $\mu=(\alpha, i)$. By Lemma 6.18, $a \circ q_{\mu}-q_{\mu} \circ a \in \Sigma_{i<j \leq 2} F_{j}+E$. By Lemma 6.18 and 6.17-(6), for any $q \in F_{j}$ with $j \leq 2$ one can find $a_{\lambda} \in \Sigma_{\varphi}^{0}$ for all $\lambda \in \Delta_{j} \equiv\{\lambda \in \Delta ; \lambda=(\alpha, i)$ with $j \leq i \leq 2\}$ such that $q-\Sigma_{\lambda \in \Delta_{j}} q_{\lambda} \circ a_{\lambda} \in E$. These two facts shows the Lemma for $s=1$. Next we assume that $s>1$ and that the Lemma is true for $1, \cdots, s-1$ so that $1_{i} \in \mathbb{N}, 1 \leq i \leq s-1$ as in the statement exist. Then for any $\nu \in \Delta$ there exist $A_{\lambda j k}^{\nu}, \lambda \in \Delta^{\prime}, j=1, \cdots, l_{s-1}, k=1, \cdots, s-1, \in O p \sum_{\varphi}^{0}$ such that $\left[Q_{\nu}, A_{1} \cdots A_{s-1}\right]-$ $\Sigma_{\lambda, j} Q_{\lambda} A_{\lambda j 1}^{\nu} \cdots A_{\lambda j s-1}^{\nu} \in \mathcal{C}_{0}$, and there exist $A_{\lambda j} \in O p \Sigma_{\varphi}^{0}, \lambda \in \Delta^{\prime}, j=1, \cdots, l_{1}$ such that $\left[Q_{\mu}, A_{s}\right]-\Sigma_{\lambda, j} Q_{\lambda} A_{\lambda j} \in \mathcal{C}_{0}$. Since $\left[Q_{\mu}, A_{1} \cdots A_{s}\right]=\left[Q_{\mu}, A_{1} \cdots A_{s-1}\right] A_{s}+A_{1} \cdots$ $A_{s-1}\left[Q_{\mu}, A_{s}\right]$, the Lemma also holds for $s$ in view of Lemma 6.13.

Next corollary is important for estimation of commutators in $\$ 7$.

Corollary 6.20. Let $\tilde{\tau}_{i}, \tau_{i}, \pi_{i}, L_{i}$, for $i=1,2,3$ be as in Lemma 6.4. Let $\varphi$ and $q$ be as in Definition 6.15. Suppose that $q(t, x, \tau, \xi)=\prod_{i=1}^{3}\left(\tau-\tilde{\tau}_{i}(t, x, \xi)\right)$, $(t, x, \xi) \in \operatorname{supp} \varphi$. Then one can find $1_{s} \in N$ for any $s \in \mathbb{N}$ with $s \geq 2$ such that for any $A_{l}, \cdots, A_{s} \in O p \Sigma_{\varphi}^{0}$ there exist $A_{l j} \in O p \Sigma_{\varphi}^{0}$ and $Q_{l} \in \mathcal{C}\left(L_{i}\right), l=1, \cdots, 1_{s}$, $j=1, \cdots, s-1$, such that

$$
\left[O p^{f} q, A_{1} \cdots A_{s}\right]-\sum_{l=1}^{l_{s}} Q_{l} A_{l 1} \cdots A_{l s-1} \in C_{0} .
$$

Proof. Assume the notations $\Delta^{\prime}, q_{\lambda}$ in the statement and the proof of Lemma 6.19. By Lemma 6.19 one can find $l_{s} \in N$ such that for any $A_{j} \in O p$ $\Sigma_{\varphi}^{0}, j=1, \cdots, s$, there exist $A_{\lambda j k}=O p a_{\lambda j k}$ with $a_{\lambda j k} \in \Sigma_{\varphi}^{0}$ for $\lambda \in \Delta^{\prime}, j=1, \cdots, l_{s}$, $k=1, \cdots, s$, such that

$$
\left[O p{ }^{f} q, A_{1} \cdots A_{s}\right]-\Sigma_{\lambda, j} O p\left(q_{\lambda} \circ a_{\lambda j k}\right) A_{\lambda j 2} \cdots A_{\lambda j s} \in \mathcal{C}_{0} .
$$

Since $q_{\lambda} \circ a_{\lambda j 1} \in F_{1}+F_{2}+E$ by Lemma 6.18 , it turns out in view of Lemma 6.13 that the proof is complete if we show that

$$
O p p \in \mathcal{C}+\mathcal{C}_{0} \text { if } p \in F_{1} \cup F_{2} \text {. }
$$

To see this we may assume that $p=f^{\left(i-\alpha_{1}\right) / 2} a^{f} \tilde{\partial}^{\alpha} q$ with $a={ }^{f} \tilde{a}, \tilde{a} \in \Sigma_{1 / 2}^{d}$ with $d \in \Delta_{\alpha, i}$ and supp $\tilde{a} \subset \operatorname{supp} \varphi, 0<|\alpha| \leq i \leq 2$. We devide the argument into two cases: (1) $\alpha_{2}+\alpha_{3}>0$ (2) $\alpha_{2}=\alpha_{3}=0$. First we consider (1). From the assumption on $q, \tilde{\partial}^{\alpha} q \cdot \tilde{a}$ is a sum of the following form:

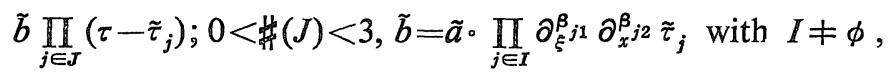

$$
\begin{aligned}
& I \cap J=\phi, \sharp(I \cup J)=3-\alpha_{l}, \sum_{j \in I} \beta_{j l}=\alpha_{l+1},\left|\beta_{j}\right|>0 \text { for } \beta_{j}=\left(\beta_{j 1}, \beta_{j 2}\right) \text {. }
\end{aligned}
$$

From the definition of $d_{\alpha, i}$ and the assumption on $\tau_{j}, b \in \Sigma_{1 / 2}^{e}$ with $e=\left(-\alpha_{1}\right.$, 
$0,0)+\#(I) / 2(0,-1,1)$. Thus, in view of $i>\alpha_{1}, p$ can be written in the form:

$$
\sum_{k \neq l} b_{k l} \pi_{k} \pi_{l}+\sum_{k=1}^{3} b_{k} \pi_{k} ; b_{k l} \in f^{1 / 2} \bigcap_{N \geq 1 / 2} A^{1 / 2, N}, b_{k} \in f^{1 / 2} \bigcap_{N \geq 1} A^{1, \alpha_{1} / 2, N},
$$

which belongs to $F+E$ by Lemma $6.4-(\mathrm{i}),(\mathrm{v})$. Next we pass to (2). $p=a$. $\sum_{\beta(I)=3-\alpha_{1}}(3-\#(I)) ! \prod_{i \in I} \pi_{i}$ and $\left.a \in{ }^{f} \sum_{1 / 2}^{(-\alpha}, 0,0\right)$. So, Lemma 6.4-(i) and (v) imply that $p \in F+E$. Tnis completes the proof.

Next we shall study the localization of the operator $Q_{\mu_{\nu}}$ in (2.17).

Definition 6.21. Let $q=\sum_{i+j \leq 3} a_{i j} \tau^{i} \xi^{j}$ with $a_{i j} \in B_{h, T}$ and $a_{30}=1$. We define for $\nu=\left(\nu_{1}, \nu_{2}\right), \mu=\left(\mu_{1}, \mu_{2}\right) \in Z_{+}^{2}$,

$$
q_{\nu \mu}=f^{\mu_{2}} \cdot \partial_{x}^{\mu} \mu^{2} f\left(\partial_{\tau}^{\nu_{1}} \partial_{\xi^{2}}^{\nu} \partial_{t}^{\mu} q\right) \cdot\langle f \xi\rangle^{\left(\nu_{2}-\mu_{2}\right) / 2}\left\langle t^{e} h f \xi\right\rangle^{\left(\nu_{1}-\mu_{1}\right)^{\prime 2}} t^{-\nu_{1}+\mu_{1}} .
$$

Lemma 6.22. $\langle f \xi\rangle^{-1 / 2} q_{\nu \mu} \in E$ when $l \in \mathbb{Z}_{+}, l+|\nu|+|\mu| \geq 3, \mu_{1} \leq 2$, and $|\nu|+|\mu|>0$. Before the proof we note that

$$
\text { for } \varepsilon \in \boldsymbol{R}, \alpha \in Z_{+}^{3},\left|\partial^{\alpha}\left\langle t^{\varepsilon} \xi\right\rangle\right| \leq C_{\alpha \varepsilon}\left\langle t^{\varepsilon} \xi\right\rangle t^{-{ }_{1}}\langle\xi\rangle^{-\alpha_{3}} \text {. }
$$

Proof of Lemma 6.22. If $q=a \tau^{i} \xi^{j}, i \leq 2, a \in B_{h, T}$, and if $\nu \leq(i, j)$, we have

$$
\begin{aligned}
& q_{\nu \mu}=\sum_{1 \leq \mu_{2}} g_{\mu_{\nu l}} \partial_{t}^{\mu} \partial_{x}^{l} a \cdot(f \xi)^{i-\nu_{2}} \cdot\langle f \xi\rangle^{\left(\nu_{2}-\mu_{2}\right) / 2}\left\langle t^{e_{h}} f \xi\right\rangle^{\left(\nu_{1}-\mu_{1}\right) / 2}
\end{aligned}
$$

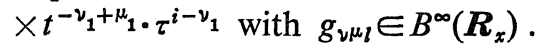

If $q=\tau^{3}$,

$$
\begin{aligned}
& q_{\nu \mu}=0 \text { when } \nu_{2}+|\mu|>0, \text { and } \\
& q_{\left(\nu_{l}, 0\right), 0}=t^{-\nu_{1}}\left\langle f^{e}{ }_{h} f \xi\right\rangle^{\nu_{1} / 2} \cdot \frac{3 !}{\left(3-\nu_{1}\right) !} \cdot \tau^{3-\nu_{1}} \text { when } \nu_{1} \leq 3 .
\end{aligned}
$$

Thus using the fact that $\partial_{t}^{\mu} \partial_{x}^{l} a \cdot t^{\mu_{1}}=t^{\min \left(\mu_{1}, 1\right) / h} \cdot b_{l}$ for some $b_{l} \in B_{h, T}$, the inequalities that $\min \left(\mu_{1}, 1\right) / h-\mu_{1} /(20 h) \geq 0$ for $2 \geq \mu_{1} \geq 0$ and that $t^{e}{ }_{h}\langle\xi\rangle \leq\left\langle t^{e}{ }^{h} \xi\right\rangle$, and (6.17) we see that when $l \in \mathbb{Z}_{+},|\nu|+|\mu|>0$, and $\mu_{1} \leq 2,\langle f \xi\rangle^{-l / 2} q_{\nu \mu}$ is a sum of symbols of the form:

$$
\begin{aligned}
& { }^{f} a \tau^{k} ; k \leq 2, \nu_{1}+\mu_{1} / 2+k \leq 3, a \in t^{-\mu_{1} / 2} \Sigma^{d} \text { with } \\
& d_{1}=-\nu_{1}, \sigma(d)=3-k-(|\nu|+|\mu|+l) / 2, d_{3}=0, \\
& \text { and } a \text { satisfies the estimate that }
\end{aligned}
$$$$
\left|\partial^{\alpha} a\right| \leq C t^{-\mu_{1} / 2-\alpha_{1}} \cdot \tilde{\psi}_{1}^{-\nu_{1}} \cdot\langle\xi\rangle^{\sigma(d)-\nu_{1} / 2-\alpha_{3}} \text {. }
$$

In view of the definition of $E$ the symbols in (6.20) are in $E$ when $l+|\nu|+|\mu|$ $\geq 3$. So the proof is complete. 
We define a similar set of operators as $\mathcal{C}$.

Definition 6.23. Let $L_{i}, i=1,2,3$ as in Lemma 6.4. We define $C^{\prime}=C^{\prime}\left(L_{i}\right)$ =the set of all operators on $\mathcal{S}_{T}$ of the form:

$$
\begin{aligned}
& \sum_{i \neq j} A_{i j} L_{i} L_{j}+\sum_{i} A_{i} L_{i}+A_{0} \\
& \text { with } A_{i j} \in \mathscr{B}^{1 / 2,1 / 2,3}, A_{i} \in \mathscr{B}^{1,1,3} \text { for } i \neq 0, A_{0} \in \mathscr{B}^{3 / 2,3 / 2,3 / 2} ;
\end{aligned}
$$

$F^{\prime}=F^{\prime}\left(L_{i}\right)=$ the set of all symbols $q$ with $O p q \in \mathcal{C}^{\prime}\left(L_{i}\right)$.

We shall show an analogous result of (6.16).

Lemma 6.24. Let $\varphi \in \Sigma^{0}$, and let $q$ be as in Definition 6.21. Let $\pi_{i}, \tau_{i}, \tilde{\tau}_{i}, L_{i}$, $\varepsilon \in(0,1)$, and $\pi=\prod_{i=1}^{3} \pi_{\imath}$ be as in Lemma 6.4. We suppose that $\varepsilon \geq 1 /(10 h)$, and that

$$
q(t, x, \tau, \xi)=\prod_{i=1}^{3}\left(\tau-\tilde{\tau}_{i}(t, x, \xi)\right),(t, x, \xi) \in \operatorname{supp} \varphi .
$$

Then, $q_{\nu \mu} \circ{ }^{f} \varphi \in F^{\prime}\left(L_{i}\right)+E$ if $0<|\nu|+|\mu|<3$.

For the proof of the lemma we make a definition.

Definition 6.25. We recall Definitions 3.7 and 6.15. With $U=\boldsymbol{R}_{T}^{3}$, and $M_{\alpha}^{(1)}=\tilde{\psi}^{-\alpha}, M_{\alpha}^{(2)}=\psi^{\prime-\alpha}$, for $\alpha \in \mathbb{Z}_{+}^{3}$ where $\psi^{\prime d}=\tilde{\psi}_{1}^{d^{1}}\langle\xi\rangle^{-d_{2} / 2} \psi_{3,1 / 2}{ }^{d_{3}}$ for $d \in \boldsymbol{R}^{3}$ by definition, we set for a positive function $Z$ on $U$,

$$
\Xi^{(i)}(Z)=\Xi\left(U, Z, M_{\alpha}^{(i)}\right), i=1,2 \text {. }
$$

Clearly,

$$
\Sigma_{1 / 2}^{d}=\Xi^{(1)}\left(\psi^{d}\right), \Sigma^{d}=\Xi^{(2)}\left(\psi^{\prime d}\right),
$$

so that

$$
\Sigma_{1 / 2}^{d} \subset t^{d_{1}} \Sigma^{\left(0, d_{1}+d_{2}, d_{3}\right)} .
$$

Indeed, using $\psi^{d}=t^{d_{1}}\left(t^{-d_{1}} \psi^{d}\right) \leq t^{d_{1}} \psi^{\left(0, d_{1}+d_{2}, d_{3}\right)}$ and Lemma 3.8-5 and 2 we have $\Xi^{(1)}\left(\psi^{d}\right)=t^{d_{1}} \Xi^{(1)}\left(t^{-d_{1}} \psi^{d}\right) \subset t^{d_{1}} \Xi\left(\psi^{\left(0, d_{1}+d_{2}, d_{3}\right)}\right)$ which implies $(6.22)$. We also have

$$
\begin{gathered}
t^{\varepsilon} \Sigma^{d+(0,0,1)} \subset \Sigma^{d+(-1,0,0)} \text { when } \varepsilon \geq 1 /(10 h), \\
t^{\varepsilon-1 / 2} B^{k, l, N} \subset B^{k, l+1 / 2, N} \text { if } k \geq l+1 / 2 \text { and } \varepsilon \geq 1 /(20 h) .
\end{gathered}
$$

Indeed, $t^{\varepsilon} \psi_{3,1 / 2}=t^{2}\left\langle t^{e}{ }_{h \xi}\right\rangle^{-1 / 2}\langle\xi\rangle \leq t^{\varepsilon-e_{h} / 2}\langle\xi\rangle^{-1 / 2} \leq t^{-1}\left\langle t^{e}{ }_{h} \xi\right\rangle^{1 / 2}$ when $\varepsilon \geq 1 /(10 h)$. So (6.23) holds by (6.21), Lemma 3.8-5 and 2. And if $d \in \Delta_{k_{0} b}$ with $k, l$ as in 
(6.24), $\psi^{\prime d}=\widetilde{\psi}_{1}^{d} \cdot\left\langle t^{e}{ }^{k} \xi\right\rangle^{\tilde{\mu}_{(d)}} \cdot\langle\xi\rangle^{\nu(d)} \leq \widetilde{\psi}^{d_{1}}\langle\xi\rangle^{\nu(d)+\tilde{\mu}_{(d)}} \leq \widetilde{\psi}_{1}^{-21}\langle\xi\rangle^{k-l}$ from the inequalities $d_{1} \geq-2 l$ and $\tilde{\mu}(d) \geq 0$, so that $t^{\varepsilon-1 / 2} \psi^{\prime d} \leq \tilde{\psi}_{1}^{-2 l}\langle\xi\rangle^{k-l-1 / 2} t^{-1 / 2+1(20 h)}$ $\langle\xi\rangle^{1 / 2} \leq \widetilde{\psi}_{1}^{-2 l-1}\langle\xi\rangle^{k-l-1 / 2}$ from $\varepsilon \geq 1 /(20 h)$. Thus, $t^{\varepsilon-1 / 2} \Sigma^{d} \subset \Sigma^{e}$ with $e=$ $(-21-1,-(k-l-1 / 2), 0) \in \Delta_{k, l+1 / 2}$, which shows (6.24) in view of the definition of $B^{k, l, N}$.

Proof of Lemma 6.24. We have

$$
\begin{aligned}
q_{\nu \mu} \circ \varphi= & \sum_{i=0}^{2} i !^{-1}\left\{\sum_{j=0}^{N} j !^{-1} \partial_{\tau}^{i} \partial_{\xi}^{j} q_{\nu \mu} \cdot D_{t}^{i} D_{x}^{j} f_{\varphi}\right. \\
& \left.+r_{N+1}\left[\partial_{\tau}^{i} \partial_{\xi}^{N+1} q_{\nu \mu}, D_{t}^{i} D_{x}^{N+1} f^{f} \varphi\right] / N !\right\} .
\end{aligned}
$$

We assume that $0<|\nu|+|\mu|<3$. Since from (6.20), $q_{\nu \mu}=\sum_{k=0}^{2}{ }^{f} a_{k} \tau^{k}$ with $a_{k}$ $(t, x, \xi) \in C^{\infty}\left(\boldsymbol{R}_{T}^{3}\right)$ satisfying $\left|\partial^{\alpha} a_{k}\right| \leq C_{\alpha} t^{-\left(3-k-\alpha_{1}\right)}\langle\xi\rangle^{5 / 2-k-\alpha_{3}}$ which implies by Lemma 3.8-5 that the notation in Definition 3.7, $a_{k} \in t^{-(3-k)} \Xi\left(\boldsymbol{R}_{T}^{3},\langle\xi\rangle^{5 / 2-k}, M_{\alpha}\right)$ with $M_{\alpha}=t^{-\alpha_{1}}\langle\xi\rangle^{-\alpha_{3}}$, the terms $r_{N+1}[\cdot]$ in (6.25) are in $E$ for large $N$, for indeed, by Corollary 4. 12-(i) with $\rho=1 / 4$ they can be written in the form $\sum_{k=0}^{2} b_{k} \tau^{k}$ with $b_{k} \in t^{-(3-k)} f^{3} \Sigma^{0}$ for $N \geq 12$, and $t^{-2} f^{3} \Sigma^{0} \subset t^{-1} B^{1 / 2,1 / 2,3}, t^{-3} f^{3} \Sigma^{0} \subset B^{3 / 2,3 / 2,3}$. A direct calculation shows that

$$
\partial_{\tau}^{i} \partial_{\xi}^{i} q_{\nu \mu}=\sum_{\substack{l \leq j \\ m \leq \mu_{2}}} C_{l n m} f^{\mu_{2}-m} \partial_{x^{2}}^{\mu-m}\left(f^{j-l}\right) f^{l} \cdot f_{l_{l m}} \cdot t^{i} q_{\left(\nu_{1}+i, \nu_{2}+j-l\right)\left(\mu_{1}, m\right)}
$$

with $a \in C^{\infty}\left(\boldsymbol{R}_{T}^{3}\right)$ satisfying

$$
\left|\partial^{\alpha} a_{l m}\right| \leq C_{\alpha}\langle\xi\rangle^{\left(-\mu_{2}+m-j-l\right) / 2-\alpha_{3}} \cdot\left\langle t^{e} h \xi\right\rangle^{-i / 2} t^{-\alpha_{1}} .
$$

By (6.26) one can easily see that

$$
\partial_{\tau}^{i} \partial_{\xi}^{j} q_{\nu \mu} \cdot D_{t}^{i} D_{x}^{j} f \varphi=\sum_{\substack{l \leq j \\ m \leq \mu_{2}}} f_{l m} q_{\left(\nu_{1}+i, \nu_{2}+l\right)\left(\mu_{1}, \mu_{2}-m\right)} \text { with }
$$

another $a_{l m} \in \Sigma^{(j-l+m)(0,1,0)}$ such that $a_{0 m}=0$ if $\mu_{2}>0$ and $m \neq 0$, and such that supp $a_{l m} \subset \operatorname{supp} \varphi$.

For non trivial terms in the summation in (6.27), $\left|\left(\nu_{1}+i, \nu_{2}+l\right)+\left(\mu_{1}, \mu_{2}-m\right)\right|$ $>0$, since when $\mu_{2}>0, a_{l m} \neq 0$ implies that $\mu_{2}-m+l>0$. So noting that $a \in f \Sigma^{0}$ and $q \in E$ implies $a q \in E$, the fact that by $\mu_{1} \leq 2$, Lemma 6.22 , and that $a_{l m} \in \Sigma^{0}$, we have only to show that

(6.28) ${ }^{f} a q_{\nu^{\mu}} \in F^{\prime}$ if $a \in \Sigma^{0}$ with supp $a \subset \operatorname{supp} \varphi$, and if $0<|\nu|+|\mu|<3$.

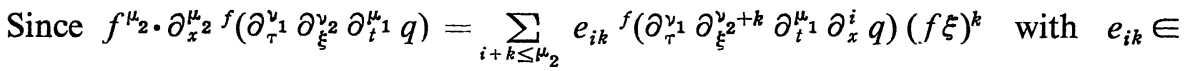
$B^{\infty}\left(R_{x}\right)$, and since supp $a \subset \operatorname{supp} \varphi,{ }^{f} a q_{\nu \mu}$ with $0<|\nu|+|\mu|<3$ can be expressed 
as a sum of the following symbols:

(1) The case $\nu_{2}+|\mu|>0$.

${ }^{f} b \cdot \prod_{s \in I}\left(\tau-\tau_{s}\right) ; 0<H(I)<3, b=a_{1} a_{2} \prod_{s \in J} \partial^{\gamma^{s}} \tau_{s}$ with

$J \neq \phi, I \cap J=\phi, \#(I \cup J)=3-\nu_{1}, \sum_{s \in J} \gamma^{s}=\left(\mu_{1}, i, \nu_{2}+k\right)$ with

$i+k \leq \mu_{2}, \gamma^{s} \neq \phi, a_{1}=a g \tilde{\psi}^{-\nu_{1}}$ with $g \in B^{\infty}\left(\boldsymbol{R}_{x}\right), a_{2}=\tilde{\psi}_{1}^{\mu_{1}\langle\xi\rangle \nu^{\left(\nu_{2}-\mu_{2}\right) / 2} \xi^{k}}$.

(2) The case $\nu_{2}=|\mu|=0 .\left(\nu_{1}=|\mu|+|\nu|>0\right.$ then.)

$$
{ }^{f} b \prod_{s \in I}\left(\tau-\tau_{s}\right) ; \#(I)=3-\nu_{1}, b=a t^{-\nu_{1}}\left\langle t^{e}{ }_{h} \xi\right\rangle^{\nu_{1} / 2} .
$$

We consider the case (1) first. We set $b_{1}=a_{2} \prod_{s \in J} \partial^{\gamma^{s}} \tau_{s}$ and $b_{2}=\prod_{s \in J} b_{2 s}$ with $b_{2 s}=\tilde{\psi} \gamma_{l}^{\gamma^{s}} \cdot\langle\xi\rangle^{\left(\gamma_{3}^{s}-\gamma_{2}^{s}\right) / 2} \partial^{\gamma^{s}} \tau_{s}$. From (6.17) we have $\left\langle t^{e}{ }_{h} \xi\right\rangle^{-\gamma_{1}^{s} / 2}\langle\xi\rangle^{\left(\gamma_{3}^{s}-\gamma_{2}^{s}\right) / 2} \in$

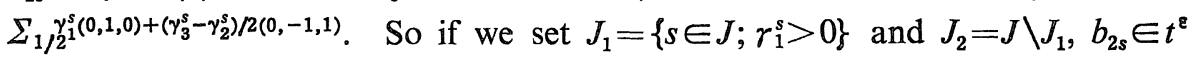
$\Sigma_{1 / 2}^{(0,0,1)}$ for $s \in J_{l}$ and $b_{2 s} \in \Sigma_{1 / 2}^{(0,-1,1) / 2}$ for $s \in J_{2}$ in view of (6.22) and Lemma3. $8-2$. Hence, by (6.23) and Lemma $3.8-2, b_{2 s} \in \Sigma^{(-1,0,0)}$ for any $s \in J$ which implies $b_{2} \in \Sigma^{(J)(-1,0,0)}$. Since $a_{2}=b_{0} b_{2}$ with $b_{0}=\xi^{k}\langle\xi\rangle^{-k}\langle\xi\rangle^{\left(i+k-\mu_{2}\right) / 2} \in \Sigma^{0}$, we have $a_{2} \in \Sigma^{m(J)(-1,0,0)}$, and therefore $b \in \Sigma^{(3-\sharp(I))(-1,0,0)}$. We have shown that ${ }^{f} a q_{\nu \mu}$ takes the following form in the case (1):

$$
\sum_{k \neq 1} b_{k l} \pi_{k} \pi_{l}+\sum_{k=1}^{3} b_{k} \pi_{k} ; b_{k l} \in f \Sigma^{(-1,0,0)}, b_{k} \in f \Sigma^{(-2,0,0)} .
$$

It is clear from (6.17) that in the case (2), ${ }^{f} a q_{\nu \mu}$ also takes the form (6.29). Now, by Lemma $6.4-(\mathrm{i})$ and (v), ${ }^{f} a q_{\nu \mu}$ with $0<|\nu|+|\mu|<3$ defines an operator of the form of the right hand side of $(v)$ in the same lemma with $k=l=1 / 2$ and $N=3$, and from (6.24), $t^{\varepsilon-1} \mathscr{B}^{3 / 2,1 / 2,3} \subset \mathscr{B}^{3 / 2,3 / 2,3}$ which completes the proof.

We shall use for proof of Proposition 2.3 the localization operator of the form $\Phi^{n}, n \in N$ with $\Phi=O p \varphi, \varphi \in \Sigma_{1 / 2}^{0}$, and $\varphi^{n}$ being an element of partitions of unity of $\boldsymbol{R}_{T}^{3}$ which is contained in $\Sigma_{1 / 2}^{0}$ and any element $\psi$ of which satisfies supp $d \psi \subset\left\{t^{\varepsilon}\langle\xi\rangle \geq c\right\}$ for some $c>0$ and $0<\varepsilon \leq e_{h}$. Such partitions of unities have been constructed in Lemma 3.2. So, we need to know that $\Phi^{n}-O p \varphi^{n}$ is small in norm.

Lemma 6.26. Let $0<\varepsilon \leq e_{h}$ and $c>0$. Let $a, b \in \Sigma_{1 / 2}^{0}(T, h)$ whose gradients supported in $D_{\varepsilon} \equiv\left\{t^{\varepsilon}\langle\xi\rangle \geq c\right\}$. Then, if $T$ is small, $O p\left({ }^{f} a{ }^{f} b\right)-O p\left({ }^{f} a{ }^{f} b\right)$ $\in t^{\varepsilon} \mathcal{A}^{0, N}$ for any $N>0$.

Proof. Since $\left\langle t^{e} h \xi\right\rangle \leq C t^{\varepsilon}\langle\xi\rangle$ in $D_{\varepsilon}$, for $\tilde{a} \in \Sigma_{1 / 2}^{0}$ supported in $D_{\varepsilon}$ 


$$
\left|\partial^{\alpha} \partial_{x}^{k} \partial_{\xi}^{l} \tilde{a}\right| \leq C_{\alpha k l} t^{\varepsilon(k+l) / 2}\langle\xi\rangle^{(k-l) / 2} \cdot \tilde{\psi}^{-\alpha} .
$$

If $T$ is small, $|\xi| \geq 1$ on $D_{\mathrm{g}}$ so that using (4.16) and Lemma $3.8-5$ one can show that for $\tilde{a}$ as above,

$$
f^{\varepsilon} \partial^{\alpha} f_{\tilde{a}} \in t^{\varepsilon\left(\alpha_{2}+\alpha_{3}\right) / 2} f_{1 / 2} \sum_{1 / 2}^{\left(-\alpha_{1}, 0,0\right)+\left(\alpha_{2}-\alpha_{3}\right) / 2(0,-1,1)} \text { for } \varepsilon>0,
$$

and that ${ }^{f} a$ also satisfies (6.30) if $k+l>0$, which imply that $\partial_{x}^{k}{ }^{f} a \cdot \partial_{\xi}^{k} f b \in$ $t^{\varepsilon f} \Sigma_{1 / 2}^{0}$ for $k>0$ and that $r_{N}\left[\partial_{\xi}^{N} f a, t^{-N^{\varepsilon / 2}} D_{x}^{N} f b\right] \in f^{N / 2} \Sigma^{0}$ in view of Lemma 3.8-5 and Corollary 4.12-(i). This completes the proof.

Corollary 6.27. Let $a$ be as in the above lemma. Then, if $T$ is small, for any $n \geq 2$ we have

$$
\left(O p^{f} a\right)^{n}-O p\left(\left(f^{f} a\right)^{n}\right) \in t^{\varepsilon} \mathcal{A}^{0, N} \text { for } N>0 .
$$

Next lemma is the one to handle the reminder term in Corollary 6.27 when the localization of $q_{\nu \mu}$ is carried out.

Lemma 6.28. Let $q$ be as in Definition 6.21. There exists $N_{0}>0$ such that if $0<|\nu|+|\mu|<3$, for any $a \in t^{\varepsilon} \mathcal{A}^{0, N}$ with $N>N_{0}$ and $\varepsilon>0$, we have

$$
q_{\nu \mu} \circ a-a \circ q_{\nu \mu}-\sum_{\substack{0<|\alpha|+|\beta|<3 \\ \beta_{1}=\mu}} a_{\alpha \beta} \circ q_{\alpha \beta} \in E
$$

with $a_{\alpha \beta} \in t^{\varepsilon} \Sigma^{0}$.

Proof. Let $a \in t^{\mathrm{e}}\left({ }^{f} \Sigma_{1 / 2}^{0}+f^{N} \Sigma^{0}\right)$ with $\varepsilon>0$ and let $\nu, \mu \in Z_{+}^{2}$ with $0<|\nu|+$ $|\mu|<3$. Using (6.26), the fact that $\partial^{\alpha} b \in t^{\varepsilon} \sum^{d-\alpha}$ (resp. $f^{\varepsilon^{\prime}} \partial^{\alpha} b \in t^{\varepsilon} \sum_{1 / 2}^{d-\alpha}$ for $\varepsilon^{\prime}>0$ ) if $b \in t^{\varepsilon} \Sigma^{d}$ (resp. $b \in t^{\varepsilon}{ }^{f} \sum_{1 / 2}^{d}$ ) which follow from (6.21), Lemma $3.8-5$, and (4.16), and the one that $f^{s}\left\langle t^{e} h \xi\right\rangle^{s}\left\langle t^{e} h f \xi\right\rangle^{-s} \in \Sigma_{1 / 2}^{0}$ for $s>0$, we have that when $i, j \in Z_{+}$and $N>\frac{i+j}{2}>0$,

$$
\begin{aligned}
& \partial_{\tau}^{i} \partial^{i} q_{\nu \mu} \partial_{i}^{i} \partial_{x}^{j} a=\sum_{\substack{l \leq j \\
m \leq \mu_{2}}} b_{l m} q_{\left(v_{1}+i, v_{2}+j-l\right)\left(\mu_{1}, m\right)} \\
& \text { with } b_{l m} \in t^{\varepsilon}\left({ }^{f} \sum_{1 / 2}^{\left(-\mu_{2}+m-l\right) / 2(0,-1,1)}+f^{(N-(i+j) / 2)} \Sigma^{\left(\mu_{2}-m+l\right)(0,1,0)}\right) \\
& \text { such that } b_{j m}=0 \text { if } \mu_{2}>0 \text { and } \mu_{2}>m .
\end{aligned}
$$

We note that from the fact that

$$
\text { if } a \in B^{k, l, N} \text { and } b \in B^{k^{\prime}, l^{\prime}, N} \text { with } N \geq k+k^{\prime}, a b \in B^{k+k^{\prime}, l+l^{\prime}, N}
$$

which follows from the fact that $d+e \in \Delta_{k+k^{\prime}, l+l^{\prime}}$ if $d \in \Delta_{k, l}$ and $e \in \Delta_{k^{\prime}, l^{\prime}}$, that $g(d)=d$ if $d \in \Delta_{k, l}$, and Fact 4.6-7, and we also note that $f^{l / 2} a \in \Sigma^{(0,1,0)}$ if 
$a \in{ }^{f} \Sigma^{(0,1,0)}$, which follows from (4.3) and (4.6). Using these facts we see if $a \in B^{0,2}$ and $q \in E, a q \in E$.

Thus, noting that $\langle f \xi\rangle^{\alpha / 2} a \in t^{\varepsilon} \mathscr{A}^{0, N}$ for $a \in{ }^{f} \sum_{l / 2}^{\alpha / 2(0,1,-1)}+f^{N} \Sigma^{(0, \infty, 0)}$ and $\alpha \geq 0$, and that $A^{0, N} \subset B^{0, N}$, and using (6.32) and Lemma 6.22 we see that

$$
\begin{aligned}
& a q_{\nu \mu} \in E \text { for } a \in{ }^{f} \sum_{1 / 2}^{j / 2(0,1,-1)}+f^{2} \sum^{(0, j, 0)} \text { with } j \in \mathbb{Z}_{+} \text {and } \\
& \nu, \mu \in \mathbb{Z}_{+}^{2} \text { with }|\nu|+|\mu|>0, j+|\nu|+|\mu| \geq 3 \text {, and } \mu_{1} \leq 2 .
\end{aligned}
$$

Since it follows from (6.20) that for $\nu, \mu \in Z_{+}^{2}$ with $0<|\nu|+|\mu|<3, q_{\vartheta \mu}=$ $\sum_{k=0}^{2} f a_{k} \tau^{k}$ with $a_{k}(t, x, \xi) \in C^{\infty}\left(\boldsymbol{R}_{T}^{3}\right)$ satisfying $\left|\partial^{\alpha} a_{k}\right| \leq C_{\alpha} t^{-(3-k)-\alpha_{1}}\langle\xi\rangle^{5 / 2-k-\alpha_{3}}$ which implies by (4.16) that $\left|\partial^{\alpha} f_{k}\right| \leq C_{\varepsilon} t^{-(3-k)-\alpha_{1}}\langle f \xi\rangle^{5 / 2-k}\langle\xi\rangle^{-\alpha_{3}+\varepsilon \alpha_{2}}$ for any $\varepsilon \in(0,1)$, there exists $M_{1} \in N$ such that for any $M \geq M_{1}$ and $i \leq 2$,

$$
\begin{aligned}
& r_{M}\left[\partial_{\xi}^{M} a, D_{x}^{M} q_{\nu \mu}\right], r_{M}\left[\partial_{\tau}^{i} \partial_{\xi}^{M} q_{\nu \mu}, D_{t}^{i} D_{x}^{M} a\right] \in E, \\
& \text { by using the fact that } D_{x}^{M} a_{k} \in t^{-(3-k)} \Sigma^{(5-M / 2)(0,-1,0)},
\end{aligned}
$$

Corollary 4.12-(i), and Lemma 4.19 for the former, and by using the fact that $a_{k} \in t^{-(3-k)} \sum_{1 / 4}^{5(0,-1 / 2,1)}$ and Corollary 4.12-(i) for the latter. In (6.31) we have that $i+j+|\nu|+|\mu|=\mu_{2}-m+l+\left|\left(\nu_{1}+i, \nu_{2}+j-l\right)+\left(\mu_{1}, m\right)\right|$, that $\left|\left(\nu_{1}+i, \nu_{2}+j-l\right)+\left(\mu_{1}, m\right)\right|>0$ if $b_{l m} \neq 0$, and that $\mu_{1} \leq 2$. So, from (6.33) we have in (6.31) that $\partial_{\tau}^{i} \partial_{\xi}^{i} q_{\nu \mu} \cdot \partial_{t}^{i} \partial_{x}^{i} a \in E$ if $i+j+|\nu|+|\mu| \geq 3$ and $N \geq \frac{i+j}{2}+2$. Thus, for $a \in t^{\varepsilon} A^{0, N}$ with $\varepsilon>0$ it follows that

$$
\begin{aligned}
& q_{\vartheta \mu} \circ a-\sum_{i+j \leq 2}(i ! j !)^{-1} D_{\tau}^{i} D_{\xi}^{j} q_{\nu \mu} \cdot \partial_{t}^{i} \partial_{x}^{i} a \in E \\
& \quad \text { if } 0<|\nu|+|\mu|<3 \text { and } N \geq\left(M_{1}+2\right) / 2+2 .
\end{aligned}
$$

Now we claim that for any $d, e \in \boldsymbol{R}^{3}$ with $d_{1}=e_{1}=0, \sigma_{1 / 2}(d) \leq 0, \sigma(e) \leq 0$, there exists $N_{0}>0$ such that

(6.36) for $N>N_{0}, a \in \sum^{f} \sum_{1 / 2}^{d}+f^{N} \Sigma^{e}$, and $\alpha, \beta \in \mathbb{Z}_{+}^{2}$ with $0<|\alpha|+|\beta|<3$, there exist $a_{\lambda \gamma} \in \Sigma$ for pairs $(\lambda, \gamma) \in Z_{+}^{2} \times Z_{+}^{2}$ with $\lambda \geq \alpha, \gamma \geq \beta$ and with $\gamma_{1}=\beta_{1}$ and $|\lambda|+|\gamma|<3$, such that $a_{\alpha \beta} \in^{f} \Sigma_{1 / 2}^{1 / 2(0,1,-1)+d}+f^{N / 2} \Sigma^{(0,1,0)+e}, a_{\lambda \gamma} \in{ }^{f} \Sigma_{1 / 2}^{d}+$ $f^{N / 2} \Sigma^{e}$ for $(\lambda, \gamma) \neq(\alpha, \beta)$, satisfying that

$$
a \circ q_{\alpha \beta}-a \cdot q_{\alpha \beta}-\sum_{(\lambda, \gamma)} a_{\lambda \gamma} q_{\lambda \gamma} \in E .
$$

It is not difficult to show Lemma 6.28 using (6.31), (6.33), (6.35), (6.36). The details are omitted and we shall prove (6.36).

Since for any $\varepsilon>0$ and any $b \in C^{\infty}\left(\boldsymbol{R}_{T}^{3}\right)$ satisfying with $d \in \boldsymbol{R}^{3}$ that $\mid \partial^{\alpha} b$ 
$(t, x, \xi) \mid \leq C_{\alpha} t^{-\alpha_{1}\langle\xi\rangle^{-\alpha_{3}} \cdot \psi^{\prime d}}$ for any $\alpha \in Z_{+}^{3}$ (see Definition 6. 25 for the definition of $\left.\psi^{\prime d}\right)$, we have by (4.16) that $f^{\varepsilon} \partial^{\alpha}{ }^{f} b={ }^{f} b_{\alpha}$ with $b_{\alpha}(t, x, \xi) \in C^{\infty}\left(\boldsymbol{R}_{T}^{3}\right)$ satisfying $\left|\partial^{\beta} b_{\alpha}\right| \leq C_{\beta} t^{\alpha_{1}-\beta_{1}}\langle\xi\rangle^{-\alpha_{3}-\beta_{3}} \cdot \psi^{\prime d}$, and since $\left|\partial^{\alpha} \psi^{\prime d}\right| \leq C_{\alpha} \psi^{\prime d} t^{-\alpha_{1}}\langle\xi\rangle^{-\alpha_{3}}$ by (6.17), for any $i \in N$

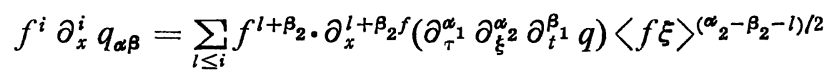

$$
\begin{aligned}
& \times\left\langle t^{e} h \xi \xi\right\rangle^{\left(\alpha_{1}-\beta_{1}\right) / 2} t^{-\alpha_{1}+\beta_{1}} \cdot{ }^{f} b_{l}
\end{aligned}
$$

with $b_{l} \in C^{\infty}\left(\boldsymbol{R}_{T}^{3}\right)$ satisfying $\left|\partial^{\alpha} b_{l}(t, x, \xi)\right| \leq C_{\alpha} t^{-\alpha_{1}}\langle\xi\rangle^{l / 2-\alpha_{3}}$. We may assume that $a={ }^{f} a_{1}+f^{N} a_{2}$ with $a_{1} \in \Sigma_{1 / 2}^{d}, a_{2} \in \Sigma^{e}$ for $d, e$ as in (6.36). Since $\partial_{\xi}^{i} a_{1}$ $\langle\xi\rangle^{l / 2} \in \Sigma^{d-1 / 2(i-l)(0,-1,1)}$ and $\partial_{\xi}^{i} a_{2}\langle\xi\rangle^{l / 2} \in \Sigma^{e-(i-l)(0,-1,0)}$ for $i, l \in Z_{+}$with $i \geq l$, we have by (6.37) that when $N>M_{1}$ and $i \in N$ with $i \leq M_{1}$ where $M_{1}$ is the one in (6.34),

$$
\begin{aligned}
\partial_{\xi}^{i} a D_{x}^{i} q_{\alpha \beta}= & \sum_{l \leq i} q_{\alpha \beta+(0, l)} b_{l} \text { for some } b_{l} \in f^{f} \sum^{d-1 / 2(i-l)(0,-1,1)} \\
& +f^{N-i} \sum^{e+(i-l)(0,1,0)}
\end{aligned}
$$

We take $N_{0}=2 M_{1}+2$ so that $N-i \geq N / 2 \geq 2$ if $N>N_{0}$. From (6.33) and the assumption on $d, e$, we have that

$$
\text { if }|\alpha|+|\beta|+l \geq 3, q_{\alpha \beta+(0, l)} b_{l} \in E .
$$

And we also have in (6.38) that

$$
\begin{gathered}
b_{0} \in{ }^{f} \sum_{1 / 2}^{d-1 / 2(0,-1,1)}+f^{N / 2} \Sigma^{e+(0,1,0)}, \\
b_{l} \in{ }^{f} \sum_{1 / 2}^{d}+f^{N / 2} \Sigma^{e} \text { for all } l .
\end{gathered}
$$

In view of (6.34), (6.38) (6.41) prove (6.36) with $N_{0}$ as in the above by the asymptotic expansion of the symbol $a \circ q_{\alpha \beta}$ with breaking off it after $M_{1}$ terms.

Next we shall study effects of localization by the partitions of unities in Lemma 3.2.

Definition 6.29. Let $X$ be a subset of $\boldsymbol{R}_{T}^{3}$ and let $a \in \Sigma$. We say $a$ belongs to $\Sigma_{\rho, X}^{d}, d \in \boldsymbol{R}^{3}$ with $d_{1} \leq 0, \rho \in[0,1 / 2]$ (resp. $\Sigma_{X}^{d}, d \in \boldsymbol{R}^{3}$ with $d_{1} \leq 0$ ) if $a$ satisfies

$$
\begin{aligned}
& \sup _{(t, x, \xi) \in X}\left|\partial^{\alpha} a\right| \psi_{1}^{-d_{1}} \cdot \psi_{2}^{-d_{2}} \cdot \psi_{3, \rho}^{-d_{3}}<\infty \\
& \text { (resp. } \left.\sup _{(t, x, \xi) \in X}\left|\partial^{\alpha} a\right| \tilde{\psi}_{1}^{-d_{1}}\langle\xi\rangle^{d_{2} / 2} \psi_{3,1 / 2}^{-d_{3}}<\infty\right)
\end{aligned}
$$

It is clear that 
$a \in \Sigma_{\rho, X}^{d}$ (resp. $\left.\Sigma_{X}^{d}\right)$ and supp $a \subset X$ implies $a \in \Sigma_{\rho}^{d}\left(\right.$ resp. $\left.\Sigma^{d}\right)$,

$a \in \Sigma_{\rho, X}^{d}$ (resp. $\Sigma_{X}^{d}$ ) implies $\partial^{\alpha} a \in \Sigma_{\rho, X}^{d-\alpha}$ (resp. $\Sigma_{X}^{d-\infty}$ )

$a \in \Sigma_{\rho_{X} X}^{d,}$ (resp. $\Sigma_{\rho_{,} X}^{d}$ ) and $b \in \Sigma_{\rho_{,} X}^{e}$ (resp. $\sum_{X}^{e}$ ) implies $a b \in \Sigma_{\rho,}^{d+e}$

(resp. $\sum_{X}^{d+e}$ ),

$a, b \in \Sigma_{\rho, X}^{d}\left(\operatorname{resp} . \Sigma_{X}^{d}\right)$ implies $a+b \in \Sigma_{\rho_{,} X}^{d}\left(\operatorname{resp} . \Sigma_{X}^{d}\right)$.

We set

$$
\Sigma_{X}=\bigcup_{d}\left(\bigcup_{\rho} \Sigma_{\rho, X}^{d} \cup \Sigma_{X}^{d}\right)
$$

Lemma 6.30. Let $a \in C^{\infty}\left(\mathbb{R}_{T}^{3}\right)$ satisfying with some $k, l \geq 0$ that $\left|\partial^{\alpha} a(t, x, \xi)\right| \leq C_{\alpha} t^{-k-\alpha_{1}}\langle\xi\rangle^{l-\alpha_{3}}$ for any $\alpha \in \mathbb{Z}_{+}^{3}$, and let $b \in \Sigma_{1 / 2}^{d}\left(\right.$ resp. $\left.\Sigma^{d}\right)$. Then, for any $N \in \mathbb{N}$ there exists $M \in \mathbb{N}$ such that

$$
{ }^{f} a \circ{ }^{f} b-\sum_{j \leq \mathbb{I}} \partial_{\xi}^{j f} a \cdot D_{x}^{j f} b / j !,{ }^{f} b \circ{ }^{f} a-\sum_{j \leq M I} \partial_{\xi}^{j f} b \cdot D_{x}^{j f} a / j ! \in t^{d_{1}-k} f^{N} \Sigma^{(0, N, 0)} .
$$

Proof. Since $\Sigma_{1 / 2}^{d} \subset \Sigma^{J_{1 / 2}{ }^{(d)}}$, it suffices to consider the latter case that $b \in \Sigma^{d}$. Since $a \in t^{-k} \sum_{1 / 4}^{l(0,-1 / 2,1)}$, Fact $4.6-7$ and Corollary 4.2-(i) show the first one. Next, from (4.16), we have for any $\varepsilon>0$ that $\left|\partial^{\alpha} f_{a}(t, x, \xi)\right| \leq$ $C_{\alpha \varepsilon} t^{-k-\alpha_{1}}\langle\xi\rangle^{l-\alpha_{3}+\varepsilon \alpha_{2}}$ so that $D_{x}^{M f} a \in t^{-k} \Sigma^{(l+\varepsilon)(0,-2,0)}$. Thus, Corollary 4.12-(ii) shows the second one. The proof is complete.

The following lemma is an easy consequence of (4.16).

Lemma 6.31. (1) Let $a \in \Sigma_{\rho, X}^{d}$ (resp. $\Sigma_{X}^{d}$ ). Then, for any $\varepsilon>0$ and $\alpha \in Z_{+}^{3}$, there exists $a_{\alpha \varepsilon} \in \Sigma_{\rho, X}^{d-\alpha}\left(\right.$ resp. $\left.\sum_{X}^{d-\alpha}\right)$ such that $f^{\varepsilon \alpha_{2} \cdot \partial^{\alpha} f} a={ }^{f} a_{\alpha \varepsilon}$ with supp $a_{\alpha \varepsilon} \subset \operatorname{supp} a$.

(2) Let $a \in C^{\infty}\left(\mathbb{R}_{T}^{3}\right)$ satisfying the estimates of the a in Lemma 6.30. Then, for any $\varepsilon>0$ and $\alpha \in \mathbb{Z}_{+}^{3}$, there exists $a_{\alpha \varepsilon} \in C^{\infty}\left(\mathbb{R}_{T}^{3}\right)$ such that $f^{\varepsilon \alpha_{2}} \cdot \partial^{\alpha} f_{a}={ }^{f} a_{\alpha \varepsilon}$ with the estimates that $\left|\partial^{\beta} a_{\alpha \varepsilon}(t, x, \xi)\right| \leq C_{\beta} t^{-k-\alpha_{1}-\beta_{1}}\langle\xi\rangle^{l-\alpha_{3}-\beta_{3}}$.

Lemma 6.32. Let $D_{t}-O p^{f} a$ with $a \in C^{\infty}\left(\mathbb{R}_{T}^{3}\right)$ satisfying the estimates for a in Lemma 6.30. Let $b \in \Sigma_{1 / 2}^{d}\left(\right.$ resp. $\left.\Sigma^{d}\right)$. Then, for any $N \in \mathbb{N}$ there exists $c \in t^{-k} \sum_{1 / 2}^{d+(l-1 / 2)(0,-1,1)}+\sum_{1 / 2}^{d+(-1,0,0)} \quad$ (resp. $t^{-k} \Sigma^{d+(l-1 / 2)(0,-2,0)}+\Sigma^{d+(-1,0,0)}$ ) with supp $c \subset \operatorname{supp} b$ such that $\left[L, O p{ }^{f} b\right]-O p{ }^{f} c \in f^{N} t^{d_{1}-k} \Sigma^{(0, N, 0)}$.

Proof. This follows from Lemma 6.30 and 6.31 in view of the equality, $\left[L, O p^{f} b\right]=O p D_{t}^{f} b-\left[O p^{f} a, O p^{f} b\right]$.

Lemma 6.33. Let $g(t, x, \xi)=d \xi^{3}+f^{1 / 2} a \xi^{2}$ with $d \in E_{h_{0} T}$ and $a \in B_{h, T^{\circ}} \quad$ Let $X$ be a subset of $\mathbb{R}_{T}^{3}$ and assume that $|g| \leq C t^{-\kappa}\langle\xi\rangle^{3 / 2}$ with $\kappa=3 / 2-1 /(3 h)$ (resp. 
$|g| \leq C\langle\xi\rangle^{2}$ ) on $X$. Then,

$$
\begin{aligned}
& \left|\partial^{\alpha} g\right| \leq C_{\alpha} t^{-\kappa-\alpha_{1}}\langle\xi\rangle^{3 / 2-\alpha_{3}} \cdot\left\langle t^{e}{ }^{h} f^{1 /(10 h)} \xi\right\rangle^{\min (|\alpha|, 1) / 2} \text { on } X \\
& \left(\text { resp. }\left|\partial^{\alpha} g\right| \leq C_{\alpha} t^{-\alpha_{1}}\langle\xi\rangle^{2+\min \left(1, \alpha_{2}\right)-\alpha_{3}} \text { on } X\right)
\end{aligned}
$$

Proof. We only prove the former case and the latter one is proved similarly. When $\langle\xi\rangle \leq 2$, the inequality clearly holds. To consider the opposite case, we note that

$$
t^{e} h^{2} f^{1 /(20 h)}\langle\xi\rangle^{1 / 2} \leq\left\langle t^{e}{ } f^{1 /(10 h)} \xi\right\rangle^{1 / 2} .
$$

When $\langle\xi\rangle \geq 2$, using $d \xi^{3}=g-f^{1 / 2} a \xi^{2},|g|^{1 / 3} \leq C\left(|t f|^{1 / 3 h}|\xi|+f^{1 / 6}|\xi|\right)$, and the assumption, we see for $\alpha \neq 0$ and $(t, x, \xi) \in X$ that

$$
\begin{aligned}
\left|\partial^{\alpha} g(t, x, \xi)\right| \leq & C_{\alpha} t^{-\kappa-\alpha_{1}}\langle\xi\rangle^{3 / 2-\alpha_{3}}\left(t^{\kappa / 3+1 /(3 h)} f^{1 /(4 h)}\langle\xi\rangle^{1 / 2}\right. \\
& \left.+t^{\kappa / 3} f^{1 / 7}\langle\xi\rangle^{1 / 6}+t^{\kappa} f^{1 / 3}\langle\xi\rangle^{1 / 2}\right) .
\end{aligned}
$$

Using $\kappa / 3+1 /(3 h)>e_{h} / 2$ and (6.43) show that the first term in the parenthesis on the right can be estimated by $f^{1 /(4 h)-1 /(20 h)}\left\langle t^{e}{ }^{h} f^{1 /(10 h)} \xi\right\rangle^{1 / 2}$, and using $2 \kappa / 3>e_{h} / 2$ and (6.43) show that the second one and the last one are estimated respectively by $f^{1 / 7-1 /(60 h)}\left\langle t^{e}{ }^{h}{ }^{1 /(10 h)} \xi\right\rangle^{1 / 6}$ and $f^{1 / 3-1 /(20 h)}\left\langle t^{e}{ }^{e} f^{1 /(10 h)} \xi\right\rangle^{1 / 2}$. Thus, we get the desired inequality for $\alpha \neq 0$ and for $\alpha=0$ nothing but the assumption. The proof is complete.

Lemma 6.34. Let $g$ and $X$ be as in Lemma 6.33 and let $b \in \Sigma_{1 / 2, X}^{e}$ (resp. $\left.\Sigma_{X}^{e}\right)$. Then, $d b \in t^{-\mathrm{k}} \Sigma_{1 / 2, X}^{-3 / 2(0,-1,1)+e}+f^{1 / 2} \Sigma_{1 / 2}^{-\left(0, \bar{X}^{1,1)+e}\right.}$ (resp. $\left.d b \in \Sigma_{\bar{X}}^{-(0,-2,0)+e}\right)$.

Proof. Let $\chi(\xi) \in C^{\infty}(\boldsymbol{R})$ with $\chi=1$ when $|\xi| \geq 1$ and $\chi=0$ when $|\xi| \leq 1 / 2$. Break up $d b$ as $\chi d b+(1-\chi) d b$. The second term $\in t^{e_{1}} \Sigma_{1 / 2, X}^{N(0,1,-1)}$ (resp. $t^{e_{1}} \Sigma_{X}^{(0, N, 0)}$ ) for any $N \in N$, and expressing the first term as $\xi^{-3} b \chi g-f^{1 / 2} a b \xi^{-1} \chi$, and using the fact that $g \in t^{-\kappa} \sum_{1 / 2, X}^{3 / 2(0,-1,1)}$ (resp. $\Sigma_{X}^{2(0,-2,0)}$ ) by Lemma 6.33, we have in view of (6.34) that $\chi d b \in t^{-\kappa} \Sigma_{1 / 2, X}^{-3 / 2(0,-1,1)}+f^{1 / 2} \sum_{1 / 2, X^{-1,1)+e}}^{-(\text {resp. }}$ $\left.\Sigma_{\bar{X}}^{(0,-2,0)+e}\right)$. This completes the proof.

Lemma 6.35. Let $L_{i}=D_{t}-a_{i}(t, x) f(x) D_{x}$ with $a_{i} \in B_{h, T}, i=1,2,3$, let $b \in{ }^{f} \sum_{1 / 2}^{0}$, and let with $c_{i}, c_{i j} \in B_{h, T}$,

$$
\begin{aligned}
R= & f^{1 / 2}(x)\left(\sum_{i=1}^{3} c_{i}(t, x) f(x) D_{x} \cdot L_{i}\right. \\
& \left.+\sum_{i \neq j} c_{i j}(t, x) L_{i} L_{j}+t^{1 / h-1} c_{0}(t, x) f(x) D_{x}\right) .
\end{aligned}
$$

Then,

$[O p b, R] \in \mathcal{C}_{0}, O p b \cdot R \in \mathcal{C}\left(L_{i}\right)+\mathcal{C}_{0}$. 
Proof. It is easy to see that $b \circ f^{1 / 2} \in f^{1 / 2 f} \Sigma_{1 / 2}^{0}+f^{N} \Sigma^{(0,1,0)}$ for all $N \in \mathbb{N}$. So, the second one follows from Lemma 4.18-(1). Next, we set $B=O p b, C_{i}=$ $f^{1 / 2} c_{i} f D_{x}$ for $i \neq 0, C_{i j}=f^{1 / 2} c_{i j}, C_{0}=t^{1 / h-1} c_{0} f^{3 / 2} D_{x}$. Then,

$$
\begin{aligned}
{[B, R]=} & \sum_{i=1}^{3}\left(\left[B, C_{i}\right] L_{i}+C_{i}\left[B, L_{i}\right]\right)+\sum_{i \neq j}\left(\left[B, C_{i j}\right] L_{i} L_{j}+C_{i j}\left[B, L_{i}\right] L_{j}\right. \\
& \left.+C_{i j}\left[L_{i},\left[B, L_{j}\right]\right]+C_{i j}\left[B, L_{j}\right] L_{i}\right)+\left[B, C_{0}\right]
\end{aligned}
$$

by Lemmas $4.18-(3)-(i)$ and 4.22 ,

$$
\left[B, C_{i j}\right] \in O p^{f} \sum_{1 / 2}^{1 / 2(0,-1,1)}+f^{N} O p \Sigma^{(0, N, 0)} \text { for } N>0
$$

by Lemma $4.18-(4)$,

$$
\left[L_{z},\left[B, L_{j}\right]\right] \in \bigcap_{N \geq 1} \mathcal{A}^{1,1, N}
$$

by Lemmas $4.18-(3)-(i)$ and 4.22 ,

$$
\left[B, C_{0}\right] \in \bigcap_{N \geq 1 / 2} t^{1 / h-1} \mathscr{A}^{1 / 2, N}
$$

by Lemma $4.18-(1)$.

Thus, from Lemma $4.18-(1)$ and that $\mathscr{A}^{k, l, N} \subset \mathcal{B}^{k, l, N}$, all terms are in (6.44) except the first ones in the each one of the second summation. Next, we note

$$
\begin{aligned}
L_{i} L_{j}= & D_{i}^{2}+d_{1}(t, x) f(x) D_{x} D_{t}+d_{2}(t, x) f(x)^{2} D_{x}^{2} \\
& +t^{1 / h-1} d_{3}(t, x) f(x) D_{x},
\end{aligned}
$$

and we let $a \in C^{\infty}\left(\boldsymbol{R}_{T}^{3}\right)$ satisfying the estimate for $a$ in Lemma 6.30. From Lemmas 6.30 and 6.31 , for $b_{1} \in{ }^{f} \sum_{1 / 2}^{1 / 2(0,1,-1)}$ we have $b_{1} \circ f a \in t^{-k}{ }^{f} \Sigma^{(l-1 / 2)(0,-2,0)}+$ $f^{M} t^{-k} \Sigma^{(0, M, 0)}$ for any $M \in N$. So, (6.45) and (6.46) show that $\left[b, C_{i j}\right] L_{z} L_{j} \in$ $\mathcal{C}_{0}$. This completes the proof.

Lemma 6.36. Let $b \in \Sigma_{1 / 2}^{d}\left(\right.$ resp. $\left.\Sigma^{d}\right)$ and let $a \in C^{\infty}\left(\boldsymbol{R}_{T}^{3}\right)$ satisfying $\left|\partial^{\infty} a\right|$ $\leq C_{\alpha} t^{-\alpha_{1}-k}\langle\xi\rangle^{l-\alpha_{3}}$. Then, there exists $c_{1}, c_{2}$, belonging to $t^{-k} \sum_{1 / 2}^{d+(l-1 / 2)(0,-1,1)}$ (resp. $\left.t^{-k} \sum^{d+(l-1 / 2)(0,-2,0)}\right)$ with $\operatorname{supp} c_{i} \subset \operatorname{supp} a \cap \operatorname{supp} b$ satisfying ${ }^{f} a \circ{ }^{f} b-$ ${ }^{f}(a \cdot b)-{ }^{f} c_{1}$ and ${ }^{f} b \circ{ }^{f} a-{ }^{f}(a \cdot b)-{ }^{f} c_{2}$ are in $f^{N} t^{d_{1}-k} \Sigma^{(0, N, 0)}$.

This is an immediate consequence of Lemma 6.30 and 6.32 .

Lemma 6.37. Let $b={ }^{f} \tilde{b}$ with $\tilde{b} \in \Sigma_{1 / 2}^{0}$. Let $L=D_{t}-f(x) a(t, x) D_{x}$ with $a \in B_{h, T}$. Then,

$$
[L, O p b] \in O p\left(\Sigma_{\widetilde{b}}^{(-1,0,0)}+\Sigma_{\widetilde{b}}^{l / 2(0,-1,1)}+f^{N} \Sigma^{(0, N, 0)}\right) \text { for } N \in \mathbb{N} .
$$


Lemma 6.38. Let $g=d \xi^{3}+f^{1 / 2} a \xi^{2}$ with $d \in E_{h, T}$ and $a \in B_{h, T}$, and let $\varphi \in \Sigma_{1 / 2}^{0}$. Assume that $|g| \leq C t^{-\kappa}\langle\xi\rangle^{3 / 2}$ on supp $\varphi$ with $\kappa$ in Lemma 6.33. Then, for any $b \in B_{h, T}$,

$$
{ }^{f} \varphi \circ f(b g) \in t^{-\kappa} \AA^{3 / 2,3} .
$$

Lemma 6.37, 6.38 are immediate consequences of Lemmas 6.30, 6.31, 6.33.

Lemma 6.39. Let $g=d \xi^{3}+f^{1 / 2} a \xi^{2}$ with $d \in E_{h, T}$ and $a \in B_{h, T}$, and let $\varphi \in \Sigma_{1 / 2}^{e}\left(\right.$ resp. $\left.\Sigma^{e}\right)$. Assume that $|g| \leq C t^{-\kappa}\langle\xi\rangle^{3 / 2}$ (resp. $\left.|g| \leq C\langle\xi\rangle^{2}\right)$ on supp $\varphi$. Then,

(1) $d(f \xi)^{l} \circ f \varphi-d(f \xi)^{l} \cdot{ }^{f} \varphi \in t^{-\kappa} f \Sigma_{1 / 2}^{(1-2)(0,-1,1)+e}+f^{1 / 2} f \Sigma_{1 / 2}^{(l-3 / 2)(0,-1,1)}$

(resp. $\left.{ }^{f} \Sigma^{(l-3 / 2)(0,-2,0)+e}\right)$ for any $l \in \mathbb{Z}_{+}$,

(2) $d(f \xi)^{l} \circ f \in \in t^{-\kappa} f \Sigma_{1 / 2}^{(l-3 / 2)(0,-1,1)+e}+f^{1 / 2} \Sigma_{1 / 2}^{(l-1)(0,-1,1)+e}$

(resp. $\left.{ }^{f} \Sigma^{(l-1)(0,-2,0)+e}\right)$ for any $l \in Z_{+}$,

(3) ${ }^{f} \varphi \circ\left(b d(f \xi)^{l}\right)-b d(f \xi)^{l} \cdot{ }^{f} \varphi \in t^{-\kappa} f \sum_{1 / 2}^{(l-2)(0,-1,1)+e}+f^{1 / 2} f \Sigma^{(l-3 / 2)(0,-1,1)+e}$

$$
+t^{e_{1}} f^{N} \Sigma^{(0, N, 0)}
$$

(resp. $\left.{ }^{f} \Sigma^{(l-3 / 2)(0,-2,0)+e}+t^{e_{1}} f^{N} \Sigma^{(0, N, 0)}\right)$ for any $b \in B_{h, T}, l \in Z_{+}$, and $N \in \boldsymbol{N}$,

(4) ${ }^{f} \varphi \circ\left(b d(f \xi)^{l}\right) \in t^{-\kappa} f \Sigma^{(l-3 / 2)(0,-1,1)}+f^{1 / 2} f \sum_{1 / 2}^{(l-1)(0,-1,1)+e}+t^{e_{1}} f^{N} \Sigma^{(0, N, 0)}$

(resp. $\left.{ }^{f} \Sigma^{(l-1)(0,-2,0)}+f^{N} t^{e_{1}} \Sigma^{(0, N, 0)}\right)$ for any $b \in B_{h, T}, l \in \boldsymbol{Z}_{+}$, and $N \in \boldsymbol{N}$.

Proof. (1) and (2) follow from Lemmas 6.30, 31-(1), 34. (3) and (4) follow from Lemmas $6.30,31,34$, and that $d \in E_{h, T}$. Indeed, for any $\varepsilon>0$ and $j \in \boldsymbol{N}$ we have for some $b_{j \varepsilon} \in B_{h, T}$ that $f^{\varepsilon} \partial_{x}^{i}\left(b d(f \xi)^{l}\right)=b_{j \varepsilon} d(f \xi)^{l}$. This completes the proof.

Lemma 6.40. Let $g=d \xi^{3}+f^{1 / 2} a \xi^{2}$ with $d \in E_{h, T}$ and $a \in B_{h, T}$, let $\varphi \in \Sigma_{1 / 2}^{0}$, and let $L=D_{t}-f(x) \lambda(t, x) D_{x}$ with $\lambda \in B_{h, T}$. Assume that $|g| \leq C t^{-\kappa}\langle\xi\rangle^{3 / 2}$ on snpp $\varphi$.

(1) For any $b \in B_{h, T}$ and any $l \in Z_{+}$, there exists $c$ belonging to $t^{-\kappa} f \Sigma_{1 / 2}^{(l-3 / 2)(0,-1,1)}+f^{1 / 2} f \Sigma_{1 / 2}^{(l-1)(0,-1,1)}+f^{N} \Sigma^{(0, N, 0)}$ such that $O{ }^{f} \varphi \cdot b d f^{l} D_{x}^{l} \cdot L=O p c \cdot L$.

(2) For any $l \in \boldsymbol{Z}_{+}$and any $N \in \boldsymbol{N}$, there exist $c_{1}, c_{2}$ belonging respectively to $t^{-\kappa} f \Sigma_{1 / 2}^{(l-3 / 2)(0,-1,1)}+f^{1 / 2} f \Sigma_{1 / 2}^{(l-1)(0,-1,1)}, t^{-\kappa} f \Sigma_{1 / 2}^{(l-3 / 2)(0,-1,1)}$

$+f^{1 / 2} f \Sigma_{1 / 2}^{(l-1)(0,-1,1)+(-1,0,0)}+t^{-\kappa} f \Sigma_{1 / 2}^{(l-1)(0,-1,1)}+f^{1 / 2} f \sum_{1,2}^{(l-1 / 2)(0,-1,1)}$

$+f^{N} \Sigma^{(0, N, 0)}$ such that

$$
d f^{l} D_{x}^{l} \cdot L \cdot O p{ }^{f} \varphi=O p c_{1} \cdot L+O p c_{2} .
$$

(3) For any $b \in B_{h, T}$, any $l \in Z_{+}$, and any $N \in \boldsymbol{N}$, there exist $c_{1}, c_{2}$ being res- 
pectively in $t^{-\kappa f} \Sigma_{1 / 2}^{(l-2)(0,-1,1)}+f^{1 / 2} f \Sigma^{(l-3 / 2)(0,-1,1)}+f^{N} \Sigma^{(0, N, 0)}$, $t^{-k}\left({ }^{f} \Sigma_{1 / 2}^{(l-3 / 2)(0,-1,1)+(-1,0,0)}+f \Sigma_{1 / 2}^{(l-1)(0,-1,1)}\right.$ $+f^{1 / 2}\left(f \sum_{1 / 2}^{(l-1)(0,-1,1)+(-1,0,0)}+{ }^{f} \Sigma_{1 / 2}^{(l-1 / 2)(0,-1,1)}\right)+f^{N} \sum^{(0, N, 0)}$ such that

$$
\left[b d f^{l} D_{x}^{l} \circ L, O p{ }^{f} \varphi\right]=O p c_{1} \circ L+O p c_{2} 。
$$

Proof. (1) follows from Lemma 6.39-(4). Next, as for (2) and (3), setting $A=d f^{l} D_{x}^{l}, B=O p{ }^{f} \varphi$ we have

$$
A L B=A[L, B]+A B L \quad \text { and } \quad b[A L, B]=b A[L, B]+[b A, B] L .
$$

So, (2) and (3) follow from Lemmas 6.36, 37, 39-(1) (3), and the fact that if $r \in \Sigma^{(0, N, 0)}$ with $N \in N, f^{l} \partial_{x}^{l}\left(f^{N} r\right) \in f^{N} \Sigma^{(0, N-l, 0)}$ for any $l \in N$. This completes the proof.

\section{§7. Lemmas on the Estimates}

Remark on notations. Constants denoted by $C$ or $C_{\dot{i}}$ in the inequalities

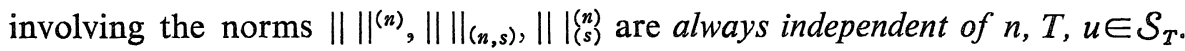
We also remark that $u$ always denotes an element of $\mathcal{S}_{T}$.

Lemma 7.1. (1) For any $k, l \in N$ with $1 \leq k, l \leq 6, n \geq 1, T \in(0,1)$,

$$
\left\|\Lambda_{k / 2} u\right\|^{(n+(l-k) / 2)} \leq C\left(n^{(k-l) / 2}\left\|\Lambda_{l / 2} u\right\|^{(n)}+n^{k / 2}\|u\|^{(n+l / 2)}\right) .
$$

(2) $\left\|D_{t} u\right\|^{(n)} \geq 2^{1 / 2} n\|u\|^{(n+1)}$ for $n \geq 1, T \in(0,1)$.

(3) Let $L_{i}, i=1,2,3$ be three operators as in Corollary 5.2. Then for small $T, n \geq 1$,

$$
\|u\|_{(6)}^{(n)} \leq C M_{n}(u) .
$$

(4) Let $L_{i}$ be as in (3). Then, for small $T, n \geq 1$,

$$
\begin{aligned}
& \sum_{i \neq j} \sum_{k=0}^{2} n^{(l-k) / 2}\left\|\Lambda_{k / 2} L_{z} L_{j} u\right\|^{(n+1-k / 2)} \\
& \quad+\sum_{i=1}^{3} \sum_{k=0}^{4} n^{l-k / 2}\left\|\Lambda_{k / 2} L_{i} u\right\|^{(n+2-k / 2)} \leq C M_{n}(u) .
\end{aligned}
$$

(5) Let $Q$ be an operator on $\mathcal{S}_{T_{0}}$ with $T_{0}$ in the statement of Corollary 5.2, and $L_{i}$ as in (3) of the form,

$$
\begin{gathered}
Q=\sum_{i \neq j} A_{i j} L_{i} L_{j}+\sum_{i=1}^{3} A_{i} L_{i}+A_{0} \text { with } \\
A_{i j} \in t^{-1} \mathscr{B}^{0,0}, A_{i} \in t^{-1} \mathscr{B}^{1 / 2,1 / 2,1 / 2} \text { for } i \neq 0, \\
A_{0} \in \sum_{k=0}^{\min (2, j)} \sum_{l=0}^{3} \sum_{j=0}^{3-1} t^{-(k / 2+3-l-j)} \mathscr{B}^{(l+j) / 2, l / 2,3 / 2)} .
\end{gathered}
$$

Then, for small $T, n \geq 2$, 


$$
\|Q u\|^{(n)} \leq C\left(n^{-1 / 2} M_{n}(u)+\|u\|_{(3)}^{(n-1 /(20 h))}\right) .
$$

(6) $n^{3 / 2-s / 2}\|u\|_{(s, n)} \leq\|u\|_{(s)}^{(n-3+s / 2)}, n \geq 4-s / 2$.

(7) $\sum_{i+j \leq s}\left\|f^{i} D_{x}^{i} D_{i}^{j} u\right\|^{(n)} \leq C\|u\|_{(2 s, n)}, s=1,2,3$.

(8) $\|u\|_{(2 s, n)} \leq C \sum_{i=0}^{s}\left\|f^{s-i} D_{x}^{s-i} D_{t}^{i} u\right\|^{(n)}, s=1,2,3$.

Proof. To see (1) one only have to apply Lemma 4.1-(i) with $\varepsilon=t n^{-1}$. (2) is a direct consequence of a simple integration by parts. (3) and (4) immediately follow from Corollary 5.2 and (1), (2) in this lemma. (5) is proved by straightforward but tedious applications of Lemmas 4.21-(1) and -(3), 7.1-(4), and 4.18-(1). We omit the details. (6) follows from (4.55) and the definition. (7) follows from Lemma 4.1-(1). (8) follows from Lemma 4.1-(1), and (2) in this lemma.

Lemma 7.2. Let $L_{i}$ be three operators as in Corollary 5.2.

(1) Let $Q \in \mathcal{C}^{\prime}\left(L_{i}\right)$. Then, for small $T, n \geq 2$,

$$
\|Q u\|^{(n)} \leq C\left(M_{n-1 /(20 h)}(u)+n^{-1 / 2} M_{n}(u)\right) .
$$

(2) Let $Q \in \mathcal{C}\left(L_{i}\right)$. Then, for small $T, n \geq 2$,

$$
\|Q u\|^{(n)} \leq C\left(M_{n-1 /(20 h)}\left(f^{1 /(20 h)} u\right)+n^{-1 / 2} M_{n}(u)\right) .
$$

(3) Let $q \in \mathcal{C}_{0} \quad$ Then, for small $T, n \geq 2$,

$$
\|Q u\|^{(n)} \leq C\left(n^{-1 / 2} M_{n}(u)+\|u\|_{(3)}^{(n-1 /(20 h))}\right) .
$$

Proof. (1) is easily proved by Lemmas 4.21, and 7.1-(3), -(4). (2) is proved by Lemmas 4.19, 7.1-(3) and -(4) for the estimation, and by Lemmas 4.18-(3), 4.22 for the commutation involving $f^{1 /(20 h)}$ and $L_{i}$. Next, to see (3) we let $Q=O p$ $q$ with $q=-\sum_{k=0}^{2} a_{k} \tau^{k}$ with $a_{k} \in \Sigma$ satisfying the condition for $a_{k}$ in the definition of $E$. Then, it is easy to see using Lemma 4.18-(1) and -(3)-(iv), and Lemma 4.22 that $Q-O p a_{2} \cdot L_{1} L_{2}$ is an operator as in Lemma 7.1-(5) which proves (3) in view of Lemma 7.1-(4). This completes the proof.

Lemma 7.3. Let $L_{i}$ be three operators as in Corollary 5.2. Set $\pi=\prod_{i=1}^{3}\left(\tau-a_{i}\right.$ $\left.(t, x) f(x) \xi-\sigma_{i}(t, x, \xi)\right)$ with the notations just above (5.16). Then we have the followings.

(1) For small $T, n \geq 1$,

$$
C^{-1} M_{n}(u) \leq\|O p \pi u\|^{(n)} \leq C M_{n}(u) .
$$


(2) Let $\varphi, q$ be as in Lemma 6.6 with $T=T_{0}$ being as in Lemma 7.1-(5) and we assume that the assumption in (ii) of that Lemma holds for $\pi, \varphi, q$ here. Then for small $T, n \geq 2$,

$$
\begin{aligned}
\|O p \pi \circ f \varphi u\|^{(n)} \leq & C\left(M_{n-1 /(20 h)}\left(f^{1 /(20 h)} u\right)+n^{-1 / 2} M_{n}(u)+\left\|O p{ }^{f} q u\right\|^{(n)}\right. \\
& \left.+\|u\|_{(3)}^{(n-1 /(20 h))}\right) .
\end{aligned}
$$

Proof. (1) is proved by Lemmas 6.4-(vi), 4.22-(1), and 7.1-(4). Next, from (5.1) and that $a_{i} \in B_{h, T_{0}}, a_{i} \xi+\tilde{\sigma}_{i}$ being in $\sum_{1 / 2}^{(0,-1,1)}\left(T_{0}, h\right)$ satisfies (6.4). Thus, by the assumption one can apply Lemma 6.6-(ii). Now, the desired inequailty follows from Lemmas 7.2-(2), -(3), 4.21-(1). This completes the proof.

Lemma 7.4. Let $\varphi \in \Sigma_{1 / 2}^{0}\left(T_{0}, h\right), q=\sum_{i+j \leq 3} a_{i j}(t, x) \tau^{i} \xi^{j}$ with $a_{30}=1$ and $a_{i j} \in$ $B_{h, T_{0}}$, and $q_{3}$ be the homogeneous part of $q$ of degree 3 in $(\tau, \xi)$. Suppose that $q_{3}=\prod_{j=1}^{3}\left(\tau-\lambda_{j} \xi\right)$ with $\lambda_{j} \in B_{h, T_{0}}$ satisfying $\inf _{t, x}\left|\operatorname{Im} \lambda_{j}\right|>0$. Then, we have the followings.

(1) For small $T$ and $n \geq 2$,

$$
n^{-1 / 2} M_{0, n}(u) \leq C\left(n^{-1 / 2}\left\|O p^{f} q u\right\|^{(n)}+\|u\|_{(4)}^{(n-1)}\right),
$$

where by definition,

$$
M_{0, n}(\cdot)=M_{n}\left(\cdot, L_{0, i}\right), L_{0, i}=D_{t}-\lambda_{i}(t, x) f(x) D_{x} .
$$

(2) For small $T$ and $n \geq 2$,

$$
M_{0, n}\left(O p^{f} \varphi u\right) \leq C\left(\left\|O p^{f} q u\right\|^{(n)}+\|u\|_{(3)}^{(n-1 /(20 h))}+\|u\|_{(4, n)}\right) .
$$

Proof. (1) We just have to apply Lemmas 7.3-(1), 7.1-(7) and -(6) in this order to the left hand side in view of the assumption on $q_{3}$.

(2) Noting the assumption on $q_{3}$ we apply Lemma 7.3-(2) for $\varphi$ with $\varphi$ here and $f^{1 /(20 h)}$ to get that

$$
\begin{aligned}
& \left\|O{ }^{f} q_{3} \circ{ }^{f} \varphi u\right\|^{(n)}+\left\|O p{ }^{f} q_{3} f^{1 /(20 h)} u\right\|^{(n)} \\
& \quad \leq C\left(M_{0, n-1 /(20 h)}\left(f^{1 /(20 h)} u\right)+n^{-1 / 2} M_{0, n}(u)+\left\|O p{ }^{f} q_{3} u\right\|^{(n)}\right. \\
& \quad+\|u\|_{(3-1 /(20 h))}^{(n-1 /(2)} .
\end{aligned}
$$

The desired inequality can be obtained by estimating the left hand side from below by Lemma 7.3, the term involving $q_{3}$ on the right by Lemma 7.1-(7), and the second term on the right by (1). The proof is complete.

Definition 7.5. We define for $\varphi \in \Sigma_{1 / 2}^{0}(T, h)$, 
$\Sigma_{k, \varphi}:=\left\{O p \varphi ; \varphi=\varphi_{1} \circ \cdots \circ \varphi_{k}\right.$ for some $\left.\varphi_{\imath} \in \Sigma_{\varphi}^{0}, i=1, \cdots, k\right\}$ for $k \in N$, $\Sigma_{0, \varphi}:=\left\{\right.$ identity on $\left.\mathcal{S}_{T}\right\}$,

where we define $\varphi_{1} \circ \cdots \circ \varphi_{k}=\varphi_{1} \circ\left(\varphi_{2} \circ \cdots \circ \varphi_{k}\right)$ for $k \geq 2$ inductively.

Lemma 7.6. Let $\varphi \in \Sigma_{1 / 2}^{0}\left(T_{0}, h\right)$ and $q=\sum_{i+j \leq 3} a_{i j} \tau^{i} \xi^{j}, a_{i j} \in B_{h, T_{0}}, a_{30}=1$. Let $L_{\imath}, \pi$ be as in Lemma 7.3 and let $M_{n}(\cdot)=M_{n}\left(\cdot, L_{i}\right)$. We suppose that the assumption on $q$ in Lemma 7.4 holds for $q$ here, and we let $M_{0, n}(\cdot)$ be defined by (7.1). Finally we let $R=D_{t}^{3}+\sum_{k=0}^{2} O p b_{k} D_{t}^{k}$ with $b_{k} \in \Sigma^{-(3-k)(0,2,0)}$. Then, we have the followings.

(1) Let $A \in \Sigma_{k, \varphi}$ with $k \geq 1$ and let $\alpha \geq 0$. Then, one can find $A^{\prime} \in \Sigma_{k, \varphi}$ such that for small $T$ and $n \geq 2$,

$$
\left|M_{n}\left(f^{\alpha} A u-A^{\prime} f^{\alpha} u\right)\right| \leq C\left(n^{-1 / 2}\left\|O p^{f} q u\right\|^{(n)}+\|R u\|^{(n)}+\|u\|_{(4)}^{(n-1)}\right) .
$$

(2) Let $k, A, \varphi$ be as in (1). Then, one can find $A^{\prime} \in \Sigma_{k, \varphi}$ such that for small T and $n \geq 2$,

$$
\left|M_{n}\left(A f^{\alpha} u-f^{\alpha} A^{\prime} u\right)\right| \leq C\left(n^{-1 / 2}\left\|O p^{f} q u\right\|^{(n)}+\|R u\|^{(n)}+\|u\|_{(4)}^{(n-1)}\right) .
$$

(3) Assume in addition that there exists $\tilde{q}=\sum_{i+j \leq 3} \tilde{a}_{i j} \tau^{i} \xi^{i}, \tilde{a}_{i j} \in B_{h, T_{0}}, \tilde{a}_{30}=1$ such that the assumption on $\pi, q, \varphi$ in Lemma 6.6-(ii) holds for $\pi, \tilde{q}, \varphi$ here. Then, for any $k \in \boldsymbol{N}$ there exists $l \in \boldsymbol{N}$ such that for any $A \in \Sigma_{k, \varphi}$ one can find $A_{i} \in \Sigma_{k-1, \varphi}$, $i=1, \cdots, l$ such that for small $T$ and $n \geq 2+1 /(20 h)$,

$$
\begin{aligned}
\left\|\left[O p^{f} \tilde{q}, A\right] u\right\|^{(n)} \leq & C\left\{\sum_{i=1}^{l}\left(M_{n-1 /(20 h)}\left(A_{i} f^{1 /(20 h)} u\right)+n^{-1 / 2} M_{n}\left(A_{i} u\right)\right)\right. \\
& \left.+n^{-1 / 2}\left\|O_{p}{ }^{f} q u\right\|^{(n)}+\|R u\|^{(n-1 /(20 h))}+\|u\|_{(4)}^{(n-1 /(20 h))}\right\} .
\end{aligned}
$$

Proof. We shall prove (1) first. Successive appliaction of Lemma 4.18-(4) shows that one can find $A^{\prime} \in \Sigma_{k, \varphi}$ with $f^{\alpha} A-A^{\prime} f^{\alpha} \in \Sigma^{(0,100)}$. Then, using Lemmas 7.3-(1) and 6.11 with $L_{0, i}$ for $L_{i}^{\prime}$ and with $L_{i}, R$ given here for $L_{i}, R$ respectively, and applying Lemma 7.1-(3) and -(4) we obtain that for small $T$ and $n \geq 1$,

$$
M_{n}\left(\left(f^{\alpha} A-A^{\prime} f^{\alpha}\right) u\right) \leq C\left(n^{-1 / 2} M_{0, n}(u)+\|R u\|^{(n)}\right) .
$$

Now, (1) follows from Lemma 7.4-(1). (2) is proved in the same way using Lemma 4.18-(4)'. We pass to (3). From Corollary 6.20 and Lemma 7.2-(2) and -(3) one can find $l \in \boldsymbol{N}$ for any $k \in \boldsymbol{N}$ such that for any $A \in \Sigma_{k, \varphi}$ one can find $A_{l}, \cdots, A_{1} \in \Sigma_{k-1, \varphi}$ such that for small $T$ and $n \geq 2$, 


$$
\begin{gathered}
\left\|\left[O{ }^{f} \tilde{q}, A\right] u\right\|^{(n) \leq} \\
C\left\{\sum_{i=1}^{l}\left(M_{n-1 /(20 h)}\left(f^{1 /(20 h)} A_{i} u\right)+n^{-1 / 2} M_{n}\left(A_{i} u\right)\right)\right. \\
+n^{-1 / 2} M_{0, n}(u)+\|u\|_{(n)}^{(n-1 /(20 h))} .
\end{gathered}
$$

Now, the desired inequality is clear in view of (1), Lemma 7.4-(1), and (4.55). This completes the proof.

Corollary 7.7. Let $\varphi, q, L_{i}, \pi$ be as in Lemma 7.6 and assume that the assumption on $\pi, q, \varphi$ in Lemma 6.6-(ii) holds for $\pi, \varphi, q$ here. Let $k, k^{\prime} \in \mathbb{N}$. Then, one can find $l \in \mathbb{N}$ such that for any $A \in \Sigma_{2 k+k^{\prime}, \varphi}$, one can find $A_{i j} \in \Sigma_{k^{\prime}, \varphi}$, $i=0, \cdots, k^{\prime}, j=1, \cdots, l$ such that for small $T$ and $n \geq k+3$,

$$
\begin{aligned}
M_{n}(A u) \leq & C\left(\left\|O p^{f} q u\right\|^{(n)}+\sum_{i=0}^{k} \sum_{j=1}^{b} n^{-(k-i) / 2} M_{n-i /(20 h)}\left(A_{i j} f^{i /(20 h)} u\right)\right. \\
& \left.+\left\|f^{k /(20 h)} u\right\|_{(4, n-k /(20 h))}+\|u\|_{(4)}^{(n-1 /(20 h))}\right) .
\end{aligned}
$$

Before the proof we prepare a lemma.

Lemma 7.8. Let $q$ be as in Lemma 7.4 and let $\alpha \geq 0$. Then, for small $T$ and $n \geq 2$,

$$
\left\|\left[O p{ }^{f} q, f^{\infty}\right] u\right\|^{(n)} \leq C\left(n^{-1 / 2}\left\|O p^{f} q u\right\|^{(n)}+\|u\|_{(4)}^{(n-1)}\right) .
$$

Proof. By the assumption on $q_{3}$ one can apply Lemma 6.10-(4) to $Q=O p$ ${ }^{f} q_{3}$ and $\alpha$. Then, using Lemmas 7.1-(4) and -(5) shows that the left hand side of the desired inequality can be dominated by $n^{-1 / 2} M_{0, n}(u)+\|u\|_{(2, n+1)}$ when $T$ is small and $n \geq 1$. Now, the desired inequality is clear from Lemmas 7.4-(1) and 7.1-(6). The proof is complete.

Proof of Corollary 7.7. We claim that for any $l \in \mathbb{N}$ with $l \geq 2$ there exists $s \in \mathbb{N}$ such that for any $A \in \Sigma_{l, \varphi}$ one can find $A_{j} \in \Sigma_{l-2, \varphi}, j=1, \cdots, s$ satisfying that with the notation $Q=O p{ }^{f} q$, for small $T$ and $n \geq 4$,

$$
\begin{aligned}
& M_{n}(A u)+\|Q A u\|^{(n)} \leq C\left\{\|Q u\|^{(n)}+\|u\|_{(4)}^{(n-1 /(20 h))}\right. \\
& \quad+\sum_{j=1}^{s}\left(\left\|A Q_{j} f^{1 /(20 h)} u\right\|^{(n-1 /(20 h))}+n^{-1 / 2}\left\|Q A_{j} u\right\|^{(n)}\right. \\
& \left.\left.\quad+M_{n-1 /(20 h)}\left(A_{j} f^{1 /(20 h)} u\right)+n^{-1 / 2} M_{n}\left(A_{j} u\right)\right)\right\} .
\end{aligned}
$$

Proof of (7.2). Let $l \in N$. Writing $A=A^{\prime} \cdot A^{\prime \prime}$ with $A^{\prime} \in \Sigma_{1, \varphi}, A^{\prime \prime} \in \Sigma_{l-1, \varphi}$ and applying Lemma 7.3-(1), -(2) to $M_{n}(A u)$ in this order shows that $M_{n}(A u)$ is dominated by the expression made from the right hand side of Lemma 7.3(2) substituting $A^{\prime \prime} u$ for $u$ when $T$ is small and $n \geq 2$. Then applying Lemma 7.6-(1) to the first term of this expression and Lemma 4.21-(2) to the last term of it, one finds that there exists $B_{1}, B_{2} \in \Sigma_{l-1, \varphi}$ such that for small $T$ and $n \geq 3$, 


$$
\begin{aligned}
& M_{n}(A u) \leq C\left(\|Q u\|^{(n-1 /(20 h))}+\|u\|_{(4)}^{(n-1 /(20 h))}\right. \\
& \quad+\sum_{j=1}^{2}\left(\left\|Q B_{j} u\right\|^{(n)}+M_{n-1 /(20 h)}\left(B_{j} f^{1 /(20 h)} u\right)+n^{-1 / 2} M_{n}\left(B_{j} u\right)\right) .
\end{aligned}
$$

Lemma 7.6-(3) shows that when $l \geq 2$, thee there exists $s^{\prime} \in \boldsymbol{N}$ and $B_{j}^{\prime} \in \Sigma_{l-1, \varphi}$, $B_{j}^{\prime \prime} \in \Sigma_{l-2, \varphi}, j=1, \cdots, s^{\prime}$ satisfying that for small $T$ and $n \geq 2$,

$$
\begin{aligned}
& \|Q A u\|^{(n)}+\sum_{j=1}^{2}\left\|Q B_{j} u\right\|^{(n)} \leq C\left[\sum _ { j = 1 } ^ { s ^ { \prime } } \left\{M_{n-1 /(20 h)}\left(B_{j}^{\prime} f^{1 /(20 h} u\right)\right.\right. \\
& \left.\quad+M_{n-1 /(20 h)}\left(B_{j}^{\prime \prime} f^{1 /(20 h)} u\right)+n^{-1 / 2}\left(M_{n}\left(B_{j}^{\prime} u\right)+M_{n}\left(B_{j}^{\prime \prime} u\right)\right)\right\} \\
& \left.\quad+\|Q u\|^{(n)}+\|u\|_{(4)}^{(n-1 /(20 h))}\right] .
\end{aligned}
$$

(7.2) follows by first applying (7.3), (7.4) in this order to the left hand side of (7.2), and again to terms involving $B_{j}, B_{j}^{\prime}$, and finally using Lemmas 7.6-(2), 7.8, 4.21-(2) for estimating a term of the form $M_{n-1 /(10 h)}\left(B f^{1 /(10 h)} u\right)$ with $B \in \Sigma_{l-2, \varphi}$ by a term of the form $M_{n-1 /(20 h)}\left(B^{\prime} f^{1 /(20 h)} u\right)+\|Q u\|^{(n)}+\|u\|_{(4)}^{(n-1 /(20 h))}$ with $B^{\prime} \in \Sigma_{l-2, \varphi}$. This proves (7.2).

Using (7.2) iteratively with the aid of Lemmas 7.8 and 4.21-(2) we get that for any $k, k^{\prime} \in N$ there exists $s \in N$ satisfying that for small $T$ and $n \geq k+3$,

$$
\begin{aligned}
& M_{n}(A u) \leq C\left(\|Q u\|^{(n)}+\|u\|_{(4)}^{(n-1 /((20 h))}\right. \\
& \quad+\sum_{i=0}^{k} \sum_{j=1}^{s} n^{-i / 2}\left(\left\|Q A_{i j} f^{(k-i) /(20 h)} u\right\|^{(n-(k-i) /(20 h))}\right. \\
& \left.\left.\quad+M_{n-(k-i) /(20 h)}\left(A_{i j} f^{(k-i) /(20 h)} u\right)\right)\right) .
\end{aligned}
$$

On the other hand for any $A \in \Sigma_{l, \varphi}, l \geq 1$, it follows from Lemmas 7.4-(2), 7.1-(6) and -(7), and 4.21-(2) that one can find $A^{\prime} \in \Sigma_{l-1, \varphi}$ satisfying that for small $T$ and $n \geq 2$,

$$
\|Q A u\|^{(n)} \leq C\left(\left\|Q A^{\prime} u\right\|^{(n)}+\|u\|_{(4)}^{(n-1 /(20 h))}+\|u\|_{(4, n)}\right) .
$$

So, iteration of this process yields that for any $A \in \Sigma_{l, \varphi}, l \geq 1$ we have that for small $T$ and $n \geq 2$,

$$
\|A Q u\|^{(n)} \leq C\left(\|Q u\|^{(n)}+\|u\|_{(4)}^{(n-1 /(20 h))}+\|u\|_{(4, n)}\right) .
$$

Applying this and Lemma 7.8 to terms in the summation in (7.5) we get that

$$
\begin{aligned}
\sum_{i=0}^{k} & \sum_{j=1}^{s} n^{i-/ 2}\left\|Q A_{i j} f^{(k-i) /(20 h)} u\right\|^{(n-(k-i) /(20 h))} \\
& \leq C_{1}\left(\sum _ { i = 0 } ^ { k } n ^ { - i / 2 } \left(\left\|Q f^{(k-i) /(20 h)} u\right\|^{(n-(k-i) /(20 h))}\right.\right. \\
& \left.+\left\|f^{(k-i) /(20 h)} u\right\|_{(4, n-(k-i) /(20 h))}+\|u\|_{(4)}^{(n-1 /(20 h))}\right) \\
& \leq C_{2}\left(\|Q u\|^{(n)}+\|u\|_{(4)}^{(n-1 /(20 h))}+\left\|f^{h /(20 h)} u\right\|_{(4, n-k /(20 h))}\right)
\end{aligned}
$$


This proves Corollary 7.7.

Throughout the following discussions in this section we assume the notations and the assumptions in $\S 3$ and that $p_{3}$ there satisfies the assumption for $q_{3}$ in Lemma 7.4. Thus, $p_{3}(t, x, 1, \zeta(t, x))^{-1} q$ with in (3.6) also satisfies the same assumption, and we assume the notation (7.1) with it. We fix some $T_{0} \in(0,1)$ as in Lemma 3.2 and we remark that the symbols of operators always are in $\mathcal{Q}_{T_{0}}$ and $u$ always $\in \mathcal{S}_{T}, T \in\left(0, T_{0}\right)$.

Note that

$$
\left(1-\mu_{l} \zeta\right)^{3}\left(1+A_{0 l}\right)=p_{3}(t, x, 1, \zeta) .
$$

We set noting (3.17)

$$
\begin{aligned}
& \tilde{L}_{l}=O p\left(\left(1-\mu_{1} \zeta\right)^{-1} f_{l}\right) \\
& L_{l}^{\prime}=\tilde{L}_{l}+O p\left(\left(1+A_{0 l}\right)^{-1}\left(1+\mu_{l} \zeta\right)^{-1}\left((-1)^{l} c b_{l}+d_{l} A_{2 l}\right) f \xi\right)
\end{aligned}
$$

Recall three cases $(1) \sim(3)$ in (3.13). Using Lemma 3.2 and the notation in it we set in case (1),

$$
L_{i j}=\tilde{L}_{l(i)}-O p \tilde{\sigma}_{i j}, i \in I
$$

where

$$
\tilde{\sigma}_{i j}=\left(1-\mu_{l(i)} \zeta\right)^{-1} \sigma_{i j}
$$

In case (2) we set that

$$
\begin{aligned}
& L_{i j}=\tilde{L}_{l(i)}-O p^{f} \tilde{\sigma}_{i j} \text { for } i \in I_{1} \text {, or } i \in I_{2} \text { and } j \neq 1, \text { and that } \\
& L_{i 1}=\tilde{L}_{l(i)}-\left(1-\mu_{l(i)} \zeta\right)^{-1} c_{l} f D_{x}-O p^{f} \tilde{\sigma}_{i j} \text { for } i \in I_{2},
\end{aligned}
$$

where $\tilde{\sigma}_{i j}$ are defined by (7.10). Here, we note that $c_{l}=\left(1+A_{0 b}\right)^{-1}(-1)^{l+1} c_{l}$. See (3.59). In case (3) we set

$$
L_{i j}=\tilde{L}-O p \tilde{\sigma}^{\tilde{\sigma}_{i j}} \text { for } i \in I,
$$

where $\tilde{\sigma}_{i j}$ are defined by (7.10) and $\tilde{L}$ are done, noting $L_{1}=L_{2}$ in case (3), by

$$
\tilde{L}=\tilde{L}_{1} \text {. }
$$

Note that

$$
\operatorname{Im}\left(\mu_{l} /\left(1-\mu_{l} \zeta\right)\right)=\operatorname{Im} \mu_{l} /\left|1-\zeta \mu_{l}\right|^{2}
$$

so that $\tilde{L}_{l}, L_{l}^{\prime}, L_{i j}$ are operators as in (5.3). We set

$$
\varphi_{0 l}=2^{-1} \varphi_{0} \text { for } l=1,2 \text { in case (3), }
$$


and we also set for $N \in \boldsymbol{N}$ with $N \geq 2$,

$$
\begin{aligned}
& \varphi_{N}=\left(\sum_{i \in I} \varphi_{i}^{N}+\sum_{l=1}^{2} \varphi_{0 l}{ }^{N}\right)^{-1 / N}, \\
& \varphi_{i N}=\varphi_{i} \cdot \varphi_{N}, \varphi_{0 l N}=\varphi_{0 l} \cdot \varphi_{N} .
\end{aligned}
$$

Then, $\varphi_{i N}, \varphi_{0 l N} \in \Sigma_{1 / 2}^{0}$ in cases (1) and (2), $\in \Sigma_{0}^{0}$ in case (3), by Lemma 3.8-(3), and

$$
\sum_{i \in I} \varphi_{i N}{ }^{N}+\sum_{l=1}^{2} \varphi_{0 l N}{ }^{N}=1
$$

Lemma 7.9. We assume the above notations and assumptions. Let $Q_{\mu_{\nu}}$ be as in (2.17) with $q$ in (3.6) for $\tilde{Q}$. Then, for small $T$ and $n \geq 2$,

$$
\begin{aligned}
\sum_{0<|\nu|+\mid \mu_{\mid} \leq 2}\left\|Q_{\mu_{\nu}} u\right\|^{(n)} \leq & C\left(\sum_{i \in I} \tilde{M}_{i, n}\left(\Phi_{i N}{ }^{N-1} u\right)+\sum_{l=1}^{2} \tilde{M}_{0 l, n}\left(\Phi_{0 l N}{ }^{N-1} u\right)\right. \\
& +n^{-1 / 2}\left\|O p^{f} q u\right\|^{(n)}+\|u\|_{\left(\begin{array}{l}
(n) \\
(-1 /(20 h))
\end{array}\right)}
\end{aligned}
$$

where

$$
\begin{aligned}
& \Phi_{i N}=O p^{f} \varphi_{i N}, \Phi_{0 l N}=O p{ }^{f} \varphi_{0 l N}, \\
& M_{i, n}(\cdot)=M_{n}\left(\cdot, L_{i j}\right), M_{0 l, n}(\cdot)=M_{n}\left(\cdot, L_{1}=L_{2}=\tilde{L}_{l}, L_{3}=L_{l}^{\prime}\right), \\
& \tilde{M}_{*, n}=M_{*, n-1 /(20 h)}+n^{-1 / 2} M_{*, n}, n \geq 1+1 /(20 h) .
\end{aligned}
$$

Proof. By Lemma 3.2,

$$
\inf t^{2 \kappa / 3}\langle\xi\rangle>0 \text { on } \bigcup_{i \in I} \operatorname{supp} \varphi_{i} \cup \bigcup_{l=1}^{2} \operatorname{supp}\left|d \varphi_{0 l}\right| \text {. }
$$

So, since $0<2 \kappa / 3 \leq e_{h}$, it follows from Lemmas $6.24,6.28$, Corollary 6.27 , and Lemma 7.2-(1) and -(3) that for small $T$ and $n \geq 2$,

$$
\begin{aligned}
& \quad \sum_{0<|\mu|+|\nu| \leq 2}\left\|Q_{\mu_{\nu}} u\right\|^{(n)} \leq C\left\{\Sigma_{i} \tilde{M}_{i, n}\left(\Phi_{i N}{ }^{N-1} u\right)\right. \\
& \left.\quad+\sum_{0<|\nu|+\mid \mu_{\mid} \leq 2}\left\|Q_{\mu_{\nu}} \Phi_{0 l N}{ }^{N} u\right\|^{(n)}+n^{-1 / 2} M_{0, n}(u)+\|u\|_{(3)}^{(n-1 /(20 h))}\right\} .
\end{aligned}
$$

To handle the second summation we set

$$
q^{(l)}=\left(1-\zeta \mu_{l}\right)^{-3} L_{l}^{2}\left(L_{l}+\left(1+A_{0 l}\right)^{-1}\left((-1)^{l} c b_{l}+d_{l} A_{2 l}\right) \xi\right) .
$$

Then,

$$
q=p_{3}(t, x, 1, \zeta(t, x)) q^{(l)}+d_{l} A_{1 l} L_{l} \xi^{2}+g_{1}+f^{1 / 2}\left(p_{2}-a_{0 l} \xi^{2}\right) .
$$

If we extend the definition of $q_{\nu \mu}$ in Definition 6.21 for $q$ of the form with in it with $a_{i j} \in B_{h, T_{0}}$ and $a_{30}=$ const. by the same equality,

$$
Q_{\mu_{\nu}}=O p\left(q_{\mu_{\nu}}^{(l)}+\left(p_{3}(t, x, 1, \zeta(t, x))^{-1}\right.\right.
$$




$$
\left.\left.\times\left(d_{l} A_{1 l} L_{l} \xi^{2}+g_{l}+f^{1 / 2}\left(p_{2}-a_{0 b} \xi^{2}\right)\right)\right) \mu_{\nu}\right) .
$$

By Lemma 6.4,

$$
O p\left(q^{(l)} \mu_{\nu}\right) \Phi_{0 l N} \in \mathcal{C}\left(L_{1}=L_{2}=\tilde{L}_{l}, L_{3}=L_{l}^{\prime}\right)+\mathcal{C}_{0}, 0<|\mu|+|\nu| \leq 2 .
$$

Noting that $\left\langle t^{e_{h}} \xi\right\rangle^{-1} t^{e_{h}}\langle\xi\rangle \leq 1$ when $t \in(0,1),(6.17),(3.65)$, and the fact that $d_{l} \in E_{h, T}$, we see that when $1 \leq|\nu|+|\mu| \leq 2$,

$$
\left(p_{3}(t, x, 1, \zeta(t, x))^{-1} d_{l} A_{1 l} L_{l} \xi^{2}\right)_{\mu_{\nu}}=d_{l}\left(a_{1}{ }^{f} L_{l}+a_{2}\right)
$$

with

$$
a_{1} \in t^{-1 / 2-1 /(20 h) f} \Sigma_{0}^{3 / 2(0,0,1)}, a_{2} \in t^{-1 / 2-1 /(20 h) f} \sum_{0}^{5 / 2(0,0,1)}+t^{-1 f} \Sigma_{0}^{(0,-1,2)},
$$

and

$$
\left(p_{3}(t, x, 1, \zeta(t, x))^{-1} f^{1 / 2}\left(p_{2}-a_{0 l} \xi^{2}\right)\right)_{\mu_{\nu}} \in E .
$$

Using the equality

$$
\begin{aligned}
& d_{l}\left(a_{1}{ }^{f} L_{l}+a_{2}\right) \circ{ }^{f} \varphi_{0 l N} \\
& \quad=d_{l}\left(\left(a_{1}\left(1-\mu_{l} \zeta\right)\right) \circ{ }^{f} D_{t} \varphi_{0 l N}\right)+d_{l}\left(\left(a_{l}\left(1-\mu_{l} \zeta\right)\right) \circ{ }^{f} \varphi_{0 l N}\right) \tau \\
& \quad-d_{l}\left(\left(a_{1} \mu_{l} f^{\prime} \xi\right) \circ{ }^{f} \varphi_{0 l N}\right)+d_{l}\left(a_{2} \circ{ }^{f} \varphi_{0 l N}\right)
\end{aligned}
$$

the fact that (3.19) holds, Lemmas 6.31-(1), 6.33, 6.34, and Corollary 4.12-(i) with $\rho=1 / 4$, we see by asymptotic expansion that

$$
d_{l}\left(a_{1}^{f} L_{l}+a_{2}\right){ }^{f} \varphi_{0 l N} \in E .
$$

Similarly, using (3.19), Lemma 6.33, and Leibniz rule we see $g_{l} \in t^{-k} \sum_{\text {supp } \varphi_{0 l}}^{3 / 2(2,-2)}$ so that Lemma 6.31-(1) and Leibniz rule show that $f^{\nu_{2}} \cdot \partial_{t, x}^{\nu}{ }^{f}\left(\partial_{\xi}^{\mu} g_{l}\right)={ }^{f} g_{l}^{\mu \nu}$ with $g_{l}^{\mu \nu} \in t^{-\kappa} \sum_{\text {supp } \varphi_{0 l}}^{3 / 2(0,0)-\left(\nu_{1}, \nu_{2}, \mu_{2}\right)}$ for $\nu, \mu \in \mathbb{Z}_{+}^{2}$. So, (6.17) and Leibniz rule show that $\left(g_{l}\right)_{\mu_{\nu}} \in t^{-\kappa} f \sum_{\operatorname{supp} \varphi_{0 l}}^{3 / 2(0,0)}$. Thus, since by (6.17) and Lemma 6.31-(2) $\left(g_{l}\right)_{\mu_{\nu}}$ $={ }^{f} \tilde{g}_{l}$ with $\left|\partial^{\alpha} \tilde{g}_{l}\right| \leq C_{\alpha}\langle\xi\rangle^{3-\alpha_{3}}$, we have $\left(g_{l}\right)_{\mu_{\nu}}{ }^{\circ} \varphi_{0 l N} \in t^{-k}{ }^{f} \Sigma^{3 / 2(0,-2,0)}+f^{3 / 2} \Sigma^{0}$ by Lemmas 6.30, 6.31. Since $t^{-1 / 2+1 /(20 h)}\langle\xi\rangle^{1 / 2} \leq t^{-1}\left\langle t^{e} h \xi\right\rangle^{1 / 2}$ when $t \leq 1$, by Lemmas $3.8-2,3.8-5$, and (6.21) we have $t^{-\kappa} f \Sigma^{3 / 2(0,-2,0)} \subset B^{3 / 2,3 / 2,3 / 2}$. Hence,

$$
\left(g_{l}\right)_{\mu \nu} \circ{ }^{f} \varphi_{0 l N} \in E .
$$

Therefore, by $(7.19) \sim(7.22)$, Lemmas 6.13 , and $7.1-(1)$ and $-(3)$ it follows that for small $T, n \geq 2$,

$$
\begin{aligned}
\sum_{1 \leq|\nu|+\mid \mu_{\mid} \leq 2}\left\|Q_{\mu_{\nu}} \Phi_{0 l N}{ }^{N} u\right\|^{(n)} \leq & C\left(\tilde{M}_{0 l, n}\left(\Phi_{0 l N}{ }^{N-1} u\right)+n^{-1 / 2} M_{0, n}(u)\right. \\
& \left.+\|u\|_{(3)}^{(n-1 /(20 h))}\right)
\end{aligned}
$$


Substituting (7.23) into (7.18), estimating $n^{-1 / 2} M_{0, n}(u)$ by Lemma 7.4-(1), and using (4.55) we get the desired inequality. The proof is complete.

\section{§8. The Derivation of (2.13) and (2.14)}

\section{$\S \S 1$. The Derivation of (2.13-1) and (2.14-1)}

(2.13-1) is nothing but Proposition 5.1. On the other hand, noting that $\left\langle t^{e}{ }_{h} \xi\right\rangle^{-1} t^{e}{ }^{e}\langle\xi\rangle \leq 1$ for $t \in(0,1)$, and (6.17), we see that

$$
Q_{\mu_{\nu}} \in O p^{f} \Sigma^{(-1,0,0)} \text { when }|\nu|+|\mu|=1 .
$$

Thus, (2.14-1) follows from Lemma 4.21 and Proposition 5.1.

\section{$\S \S 2$. The Derivation of (2.13-2) and (2.14-2)}

From the assumption of Proposition 2.3,

$$
p_{2}=\prod_{i=1}^{2}\left(\tau-\lambda_{i} \xi\right) \text { with } \lambda_{i} \in B_{h, T_{0}} \text { satisfying } \inf \left|\operatorname{Im} \lambda_{i}\right|>0 .
$$

(2.13-2) is an immediate consequence of Corollary 5.2 and Lemma 6.10-(1), and similarly as $\S \S 1$,

$$
\begin{gathered}
Q_{\mu_{\nu}}=\sum_{j=1}^{2} O p\left(a_{j}\left(\tau-\left(1-\lambda_{j} \zeta\right)^{-1} \lambda_{j} f \xi\right)\right) \bmod O p^{f} \Sigma^{(-1,0,0)} \\
\text { with } a_{j} \in{ }^{f} \Sigma^{(-1,0,0)} \text { when }|\mu|+|\nu|=1 .
\end{gathered}
$$

(2.14-2) is an easy consequence of Lemmas 4.18-(3), 4.21-(1), and Corollary 5.2-(i).

\section{$\S \S 3$. The Derivation of $(2.13-3)$ and (2.14-3)}

$p_{3}$ and $p_{2}$ in Proposition 2.3 satisfy the assumptions for those in $\S 3$. So, identifying the latters with formers respectively, we use the notations, the assumptions, the conventions, and the results in $\S 3$ and in $\$ 7$ after Corollary 7.7.

Here, again, we remark that all symbols of operators are in $\mathcal{Q}_{T_{0}}$, where $T_{0} \in(0,1)$ is as in Lemma 3.2, that $u$ denotes an element in $\mathcal{S}_{T}$ with $T \in\left(0, T_{0}\right)$.

The proof of (2.13) and (2.14) is devided into three cases (1) (3) in (3.13). These cases are considered in order from (1).

Remark on notation. In $\S 8, \S 9$, the constants denoted by $C$ or $C_{i}$ in the estimates are always independet of $T, n, u \in \mathcal{S}_{T}$ as in $\S 7$.

Proposition 8.1. Assume that (1) of (3.13) holds. Then, there exists $r_{0}$, $n_{0} \in \boldsymbol{N}$ with $n_{0} \geq r_{0}+1$ such that for small $T$ and $n \geq n_{0}$, 


$$
\begin{aligned}
& \left\|f^{r_{0}} u\right\|_{\left(4, n-r_{0}\right)}+\|u\|_{(6)}^{(n)} \leq C\|Q u\|^{(n)}, \\
& \left\|Q_{\mu_{\nu}} u\right\|^{(n)} \leq C\left(\|Q u\|^{(n-1 /(20 h))}+n^{-1 / 2}\|Q u\|^{(n)}\right) \text { when } 1 \leq|\nu|+|\mu| \leq 2 .
\end{aligned}
$$

From the assumptions (2.7), (2.8), in all cases (1) (3),

$$
\begin{aligned}
& p_{3}(t, x, \tau, \zeta(t, x) \tau+\xi)=p_{3}(t, x, 1, \zeta(t, x)) \prod_{i=1}^{3}\left(\tau-\lambda_{i}(t, x) \xi\right) \text { with } \\
& \lambda_{i} \in B_{h, T_{0}} \text { satisfying that } \\
& \quad \inf _{i, x}\left|\operatorname{Im} \lambda_{i}\right|>0 \text { and } \lambda_{i}-\lambda_{j} \in E_{h, T_{0}} \text { for any } i, j .
\end{aligned}
$$

Proof of Proposition 8.1. It suffices to prove that two inequalities in the statement replacing $Q$ on the right hand side by $p_{3}(t, x, 1, \zeta(t, x))^{-1} O{ }^{f}{ }^{f} q$ with $q$ in (3.6). In the following we denote this operator by $Q$. In case (1),

$$
\lambda_{i}-\lambda_{j} \in E_{h, T_{0}} \backslash\{0\} \text { if } i \neq j .
$$

So, from Lemma 6.10-(3) one can find $r_{0} \in N$ such that

(8.3) for any $0 \leq i \leq 2$ there exist $c_{k l}, c_{k} \in B_{h, T_{0}}, 0 \leq k \leq 3,1 \leq l \leq 3$ satisfying

$$
t^{r_{0}} \cdot f^{i} D_{x}^{i} D_{l}^{2-i} f^{r_{0}}=\sum_{l \neq k} c_{k l} L_{0, l} L_{0, k}+\sum_{k=1}^{3} c_{k} L_{0, k}+c_{0} .
$$

So, by Lemma 7.1-(4), for small $T$ and $n \geq r_{0}+1$,

$$
\sum_{i=0}^{2}\left\|f^{i} D_{x}^{i} D_{t}^{2-i} f^{r_{0}} u\right\|^{\left(n-r_{0}\right)} \leq C_{0} n^{-1 / 2} M_{0, n}(u),
$$

where by our convention in the beginning of $\S \S 3$ we set

$$
M_{0, n}(\cdot)=M_{n}\left(\cdot, L_{0, i}\right), L_{0, i}=D_{t}-\lambda_{i} f D_{x} .
$$

Thus, from Lemma 7.1-(8), for small $T$ and $n \geq r_{0}+1$,

$$
\left\|f^{r_{0}} u\right\|_{\left(4, n-r_{0}\right)} \leq C_{1} n^{-1 / 2} M_{0, n}(u) \leq C_{2}\left(n^{-1 / 2}\|Q u\|^{(n)}+\|u\|_{(4)}^{(n-1)}\right),
$$

where the right inequality follows from Lemma 7.4-(1). Next, from (3.20), Corollary 6.27, Lemma 4.21, (4.55), Lemma 7.1-(3) we have that for small $T$ and $n \geq 2$,

$$
\begin{aligned}
& \|u\|_{(6)}^{(n)} \leq C_{3}\left(\sum_{i \in I} M_{i, n}\left(\Phi_{i N}{ }^{N} u\right)+\sum_{l=1}^{2} M_{0 l, n}\left(\Phi_{0 l N}{ }^{N} u\right)+T^{2 \kappa / 3}\|u\|_{\left(\begin{array}{l}
(n) \\
6
\end{array}\right)}\right), \\
& \text { where } \Phi_{i N}=O{ }^{f} \varphi_{i N}, \Phi_{0 l N}=O{ }^{f} \varphi_{0 l N} .
\end{aligned}
$$

We set

$$
N_{0}=20 h\left(2 r_{0}+4\right) \text {. }
$$

Lemma 8.2. Let $N_{j} \in N, j=1,2$ with $N_{1}=2 N_{2}+1 \geq 2 N_{0}+1$, and let $\Phi \in$ 
$\Sigma_{1, \varphi_{i}}$ for $i \in I$. Set $J=J(u)=M_{i, n}\left(\Phi^{N_{1}} u\right)$ for $u \in \mathcal{S}_{T}$. Then, for small $T$ and $n \geq N_{2}+3$,

$$
J \leq C\left(\|Q u\|^{(n)}+\|u\|_{(4)}^{(n-1 /(20 h))}+\left\|f^{r_{0}} u\right\|_{\left(4, n-r_{0}-3\right)}\right) .
$$

Proof. We apply Corollary 7.7 for $J$ taking $k=N_{2}$ and $k^{\prime}=1$ to obtain that there exist $l_{0} \in N$ and $B_{j} \in \Sigma_{1, \varphi_{i}}, j=1, \cdots, l_{0}$ such that for small $T$ and $n \geq N_{2}+3$,

$$
\begin{aligned}
J \leq & C_{1}\left(\|Q u\|^{(n)}+\sum_{r=0}^{N_{2}} \sum_{j=1}^{l_{0}} n^{-\left(N_{2}-r\right) / 2} M_{i, n-r /(20 h)}\left(B_{j} f^{r /(20 h)} u\right)\right. \\
& \left.+\left\|f^{2 r_{0}+4} u\right\|_{\left(4, n-2 r_{0}-4\right)}+\|u\|_{(4)}^{(n-1 /(20 h))}\right) .
\end{aligned}
$$

From (3.21), (3.22), (3.24) of Lemma 3.2, $L_{i j}$ can be expressed as $L_{i j}=O p$ $\left(\tau-{ }^{f} \tilde{\tau}_{i j}\right)$ with some $\tilde{\tau}_{i j} \in \Sigma_{1 / 2}^{(0,-1,1)}$ satisfying the condition for $\tilde{\tau}_{j}$ of Lemma 6.4 with $\varepsilon=1 /(6 h)$ and that $q=\prod_{j=1}^{3}\left(\tau-\tilde{\tau}_{i j}(t, x, \xi)\right)$ for $(t, x, \xi) \in \operatorname{supp} \varphi_{i}$. So, it follows from Lemma 6.6, (5.1), Lemma 4.18-(1) by setting $\pi^{(i)}=\prod_{j=1}^{3}\left(\tau-{ }^{f} \tilde{\tau}_{i j}\right)$ for each $i \in I$ that

$$
O p \pi^{(i)} \cdot B_{j}-B_{j} Q-\sum_{k=0}^{2} A_{i j k} D_{t}^{k} \in \mathcal{C}_{0}
$$

with some $A_{i j 2} \in \mathcal{A}^{1 / 2,1 / 2,3}, A_{i j 1} \in \mathcal{A}^{3 / 2,1 / 2,3}+\mathcal{A}^{1,1,3}$,

$$
A_{i j 0} \in \mathcal{A}^{5 / 2,1 / 2,3}+\mathcal{A}^{2,1,3}+t^{1 /(6 h)-1} \mathscr{A}^{3 / 2,1 / 2,3} .
$$

By Lemmas 7.2-(3) and 7.4-(1), for any $R \in \mathcal{C}_{0}$ there exists $C>0$ such that for small $T$ and $n \geq 2$,

$$
\|R u\|^{(n)} \leq C\left(n^{-1 / 2}\|Q u\|^{(n)}+\|u\|_{(4)}^{(n-1 /(20 h))}\right) .
$$

The summation in (8.7) can be setimated by using Lemma 4.2-(1), -(2), and (4.55) as

$$
\sum_{k=0}^{2}\left\|A_{i j k} D_{t}^{k} u\right\|^{(n)} \leq C_{2}\left(\sum_{k=0}^{2}\left\|\Lambda_{5 / 2-k} D_{t}^{k} u\right\|^{(n+1 / 2)}+\left\|D_{t}^{k} u\right\|^{(n+3-k)}\right)
$$

for small $T$ and $n \geq 2$.

From (8.7) (8.9) we get that for small $T$ and $n \geq 2$,

$$
\begin{aligned}
& \left\|\left(O p \pi^{(i)} \cdot B_{j}-B_{j} Q\right) u\right\|^{(n)} \leq C_{3}\left(n^{-1 / 2}\|Q u\|^{(n)}+\|u\|_{(4)}^{n-1 /(20 h))}\right. \\
& \left.\quad+\sum_{k=0}^{2}\left(\left\|\Lambda_{5 / 2-k} D_{t}^{k} u\right\|^{(n+1 / 2)}+\left\|D_{t}^{k} u\right\|^{(n+3-k)}\right)\right) .
\end{aligned}
$$

Since for $0 \leq r \leq N_{2}$ and $n \geq N_{2}+3$,

$$
n-r /(20 h) \geq N_{2}+3-N_{2} /(20 h) \geq(20 h-1)\left(2 r_{0}+4\right)+3,
$$


we have that for $0 \leq r \leq N_{2}$, small $T$, and $n \geq N_{2}+3$,

$$
\begin{aligned}
& \left\|O p \pi^{(i)} B_{j} u\right\|^{\left(n_{r}\right)} \leq C_{4}\left(\|Q u\|^{\left(n_{r}\right)}+n_{r}^{-1}\|Q u\|^{\left(n_{r}\right)}+\|u\|_{(4)}^{\left(n_{r+1}\right)}\right. \\
& \left.\quad+\sum_{k=0}^{2}\left(\left\|\Lambda_{5 / 2-k} D_{i}^{k} u\right\|^{\left(n_{r}+1 / 2\right)}+\left\|D_{b}^{k} u\right\|^{\left(n_{r}+3-k\right)}\right)\right)
\end{aligned}
$$

with the notation that

$$
n_{q}=n-r /(20 h) \text { for } r \in \mathbb{Z}_{+} \text {and } n \geq 1+r /(20 h) \text {. }
$$

Applying Lemma 7.3-(1) to $M_{i, n_{r}}\left(B_{i} f^{r /(20 h)}\right)$ in (8.6), using (8.11), and estimating $\left\|Q f^{r /(20 h)} u\right\|^{\left(n_{r}\right)}$ by Lemma 7.8 and $\left\|f^{r /(20 h)} u\right\|_{(4)}^{\left(n_{r+1}\right)}$ by Lemma 4.21-(2) one obtain that

$$
\begin{aligned}
& M_{i, n_{r}}\left(B_{j} f^{r /(20 h)} u\right) \leq C_{5}\left(\|Q u\|^{\left(n_{r}\right)}+\|u\|_{(4)}^{\left(n_{r}+1\right)}\right. \\
& \quad+\sum_{k=0}^{2}\left(\left\|\Lambda_{5 / 2-k} D_{t}^{k} f^{r /(20 h)} u\right\|^{\left(n_{r}+1 / 2\right)}+\left\|D_{t}^{k} f^{r /(20 h)} u\right\|^{\left(n_{r}+3-k\right)}\right) \\
& \text { for } \quad 0 \leq r \leq N_{2} \text {, small } T, n \geq N_{2}+3
\end{aligned}
$$

We use the inequalities that

$$
\begin{aligned}
& \left\|\Lambda_{5 / 2-k} u\right\|^{(n)} \leq C\left(\left\|\Lambda_{1 / 2} f^{2-k} D_{x}^{2-k} u\right\|^{(n)}+\|u\|^{(n)}\right) \\
& \text { for } 0 \leq k \leq 2, n \geq 1
\end{aligned}
$$

and that

$$
\begin{aligned}
& \left\|\Lambda_{1 / 2} f^{2-k} D_{x}^{2-k} D_{i}^{k} f^{r} 0 u\right\|^{\left(n-r_{0}\right)} \leq C M_{0, n-1 / 2}(u), \\
& \left\|D_{b}^{2} f^{r_{0}} u\right\|^{\left(n-r_{0}\right)} \leq C M_{0, n-1}(u), \\
& \text { for } 0 \leq k \leq 2, \text { small, } T, n \geq r_{0}+1,
\end{aligned}
$$

where (8.13) follows from Corollary 4.13-(ii) and (8.14) is shown by using (8.3), Lemmas 7.1-(3) and -(4), 4.18, 4.21-(1), and (4.55).

When $N_{2} \geq r \geq N_{2}-2$, we have $r /(20 h) \geq 2 r_{0}+3$ and we can apply (8.13), (8.14), and Lemma 7.1-(3) to get that

$$
\begin{aligned}
& M_{i, n_{r}}\left(B_{j} f^{r /(20 h)} u\right) \leq C_{6}\left(\|Q u\|^{\left(n_{r}\right)}+\|u\|_{(4)}^{\left(n_{r+1}\right)}\right. \\
& \left.\quad+M_{0, n_{r}+r_{0}}\left(f^{r /(20 h)-r_{0}} u\right)\right) \\
& \text { for } \quad N_{2} \geq r \geq N_{2}-2 \text {, small } T, n \geq N_{2}+3 .
\end{aligned}
$$

By Lemmas 7.4-(2), 7.8, 4.21-(1), the last term on the right can be dominated by $\left.\|Q u\|^{\left(n_{r}+r_{0}\right)}+\|u\|_{(4)}^{\left(n_{r}+r_{0}-1\right)}+\|u\|_{(3)}^{n_{r}+1}+r_{0}\right)+\left\|f^{r /(20 h)-r_{0}} u\right\|_{\left(4, n_{r}+r_{0}\right)}$. Thus, noting $r /(20 h) \geq 2 r_{0}+3$ in (8.15) and using Lemma 4.21-(4) to estimate $\| f^{r /(20 h)-r_{0}}$ $u \|_{\left(4, n_{r}+r_{0}\right)}$ by $\left\|f^{r_{0}} u\right\|_{\left(4, n-r_{0}-3\right)}$ we get from (8.15) that

$$
\begin{aligned}
& M_{i, n_{r}}\left(B_{j} f^{r /(20 h)} u\right) \leq C_{7}\left(\|Q u\|^{\left(n-r_{0}-3\right)}+\|u\|_{(4)}^{\left(n-r_{0}-3\right)}+\left\|f^{r} u\right\|_{\left(4, n-r_{0}-3\right)}\right) \\
& \text { for } \quad N_{2} \geq r \geq N_{2}-2, \text { small } T, n \geq N_{2}+3 .
\end{aligned}
$$


On the other hand, when $0 \leq r \leq N_{2}-3$, we have applying Lemmas 4.21(2), 7.1-(3), (4.55), and Lemma 7.4-(1) to (8.12) that for small $T$ and $n \geq N_{2}+3$,

$$
\begin{aligned}
& n^{-\left(N_{2}-r\right) / 2} M_{i, n_{r}}\left(B_{j} f^{r /(20 h)} u\right) \leq C_{8} n^{-3 / 2} M_{i, n}\left(B_{j} f^{r /(20 h)} u\right) \leq C_{9}\left(n^{-3 / 2}\right. \\
& \left.\quad \times\|Q u\|^{(n)}+n^{-3 / 2}\|u\|_{(4)}^{(n-1 /(20 h))}+n^{-1 / 2}\|u\|_{(4)}^{(n)}\right) \leq C_{10}\left(n^{-3 / 2}\|Q u\|^{(n)}\right. \\
& \left.\quad+n^{-3 / 2} M_{0, n-1 /(20 h)}(u)+n^{-1 / 2} M_{0, n}(u)\right) \leq C_{11}\left(n^{-1 / 2}\|Q u\|^{(n)}+\|u\|_{(4)}^{(n-1)}\right) .
\end{aligned}
$$

So,

$$
\begin{aligned}
& n^{-\left(N_{2}-r\right) / 2} M_{i, n_{r}}\left(B_{j} f^{r /(20 h)} u\right) \leq C_{11}\left(n^{-1 / 2}\|Q u\|^{(n)}+\|u\|_{(4)}^{(n-1)}\right) \\
& \text { for } 0 \leq r \leq N_{2}-3 \text {, small } T \text {, and } n \geq N_{2}+3 .
\end{aligned}
$$

Now, the desired inequality follows from (8.6), (8.16), (8.17), (4.55), and Lemma 4.21-(4).

By Lemma 8.2 it follows that the term involving $\Phi_{i N}$ in (8.5) can be estimated for large odd $N$, and to estimate the term involving $\Phi_{0 l N}$ we need the following two lemmas.

Lemma 8.3. Let $m \in \mathbb{N}$ with $m \geq 2$. Then, one can find $s \in \boldsymbol{N}$ such that for any $A \in \Sigma_{m, \varphi_{0 l}}$ there exists $A_{j} \in \Sigma_{m-1, \varphi_{0 l}}, j=1, \cdots, s$ satsifying that for small $T$ and $n \geq 3$,

$$
\begin{aligned}
& M_{0 l, n}(A u) \leq C\left\{\sum_{j=1}^{s}\left(M_{0 l, n-1 /(20 h)}\left(A_{j} f^{1 /(20 h)} u\right)+n^{-1 / 2} M_{0 l, n}\left(A_{j} u\right)\right)\right. \\
& \left.+\|Q u\|^{(n)}+\|u\|_{(4)}^{(n-1 /(20 h))}\right\} \text {. }
\end{aligned}
$$

Proof. By Lemma 7.3 we have that for small $T$ and $n \geq 1$,

$$
M_{0 l, n}(A u) \leq C_{1}\left(\left\|\left[O p^{h} q^{(l)}, A\right] u\right\|^{(n)}+\left\|A \cdot O p^{f} q^{(1)} u\right\|^{(n)}\right) .
$$

We set

$$
R=O p\left\{p_{3}(t, x, 1, \zeta(t, x))^{-1} f\left(d_{l} A_{1 l} L_{l} \xi^{2}+f^{1 / 2} p_{2}-a_{0 l} f^{1 / 2} \xi^{2}\right)\right\}
$$

so that

$$
Q=O p^{f} q^{(l)}+R+O p\left(p_{3}(t, x, 1, \zeta(t, x))^{-1} f_{l}\right)
$$

Noting (3.19), we have for any $\varphi \in \Sigma_{\varphi_{0 l}}^{0}$ that

$$
\varphi^{\circ} f\left(p_{3}(t, x, 1, \zeta(t, x))^{-1} g_{l}\right) \in E \quad \text { from Lemma 6.38, }
$$

$[O p \varphi, R] \in \mathcal{C}_{0}$ from Lemmas 6.35, 40-(3), and (6.24),

Op $\varphi \cdot R \in \mathcal{C}+\mathcal{C}_{0}$ with $L_{1}=L_{2}=\tilde{L}_{l}, L_{3}=L_{l}^{\prime}$

from Lemmas 6.35, 40-(1), and (6.24) . 
Writing $A=A^{(1)} \cdots A^{(m)}$ with $A^{(i)} \in \Sigma_{1, \varphi_{0}}$, we have from (8.20) that

$$
\begin{aligned}
& A \cdot O p{ }^{f} q^{(l)}=A\left(Q-R-O p\left(p_{3}(t, x, 1, \zeta(t, x))^{-1} g_{g}\right)\right), \\
& A R=A^{(1)} R A^{(2)} \cdots A^{(m)}+A^{(1)} \sum_{j=2}^{m} A^{(2)} \cdots\left[A^{(j)}, R\right] \cdots A^{(m)} .
\end{aligned}
$$

Thus, noting (8.21), (8.22), Lemma 6.13, and using Lemma 7.2-(2) and -(3) we obiain setting $A_{1}=A^{(2)} \ldots A^{(m)}$ that for small $T, n \geq 2$,

$$
\begin{aligned}
& \left\|A \cdot O p^{f} q^{(l)} u\right\|^{(n)} \leq C_{2}\left(\|Q u\|^{(n)}+M_{0 l, n-1 /(20 h)}\left(f^{1 /(20 h)} A_{1} u\right)\right. \\
& \left.\quad+n^{-1 / 2} M_{0, n}(u)+\|u\|_{(3)}^{(n-1 /(20 h))}\right) .
\end{aligned}
$$

From Lemma 7.6-(1) there exists $A_{2} \in \Sigma_{m-1, \varphi_{0 l}}$ such that for small $T, n \geq 3$,

$$
\begin{aligned}
M_{0 l, n-1 /(20 h)}\left(f^{1 /(20 h)} A_{1} u\right) \leq & M_{0 l, n-1 /(20 h)}\left(A_{2} f^{1 /(20 h)} u\right) \\
& +C_{3}\left(\|Q u\|^{(n-1 /(20 h))}+\|u\|_{(4)}^{(n-1 /(20 h)-1)}\right) .
\end{aligned}
$$

Substituting this into (8.23) and estimating the forth term in (8.23) by Lemma 7.4-(1) we see that

$$
\begin{aligned}
\left\|A \cdot O p^{f} q^{(l)} u\right\|^{(n)} \leq & C_{3}\left(\|Q u\|^{(n)}+M_{0 l, n-1 /(20 h)}\left(A_{2} f^{1 /(20 h)} u\right)\right. \\
& \left.+n^{-1 / 2} M_{0 l, n}\left(A_{1} u\right)+\|u\|_{(4)}^{(n-1 /(20 h))}\right) .
\end{aligned}
$$

Now, applying Lemma 7.6-(3) to $\left\|\left[O p^{f} q^{(l)}, A\right] u\right\|^{(n)}$ taking $\tilde{q}=q^{(l)}$ and $R=Q$, and using (8.18) and (8.24) we see that the desired conclusion holds. This completes the proof of Lemma 8.3.

Lemma 8.4. Let $m \in N$ with $m \geq 2$ and $A \in \Sigma_{m, \varphi_{0}}$. Then, for small $T$ and $n \geq m+1$,

$$
\begin{aligned}
M_{0 l, n}(A u) \leq & C\left(\|Q u\|^{(n)}+\|u\|_{(4)}^{(n-1 /(20 h))}\right. \\
& \left.+\left\|f^{(m-1) /(20 h)} u\right\|_{(4, n-(m-1) /(20 h)}\right) .
\end{aligned}
$$

Proof. Successive use of Lemmas 8.2, 7.8, 4.21-(2) shows that there exist $s \in \boldsymbol{N}$ and $A_{1}, \cdots, A_{s} \in \Sigma_{l, \varphi_{0 l}}$ such that for small $T$ and $n \geq m+1$,

$$
\begin{aligned}
M_{0 l, n}(A u) & \\
\leq & C_{1}\left(\sum_{i=1}^{s} \sum_{j=0}^{m-1} n^{-(m-j-1) / 2} M_{0 l, n-j /(20 h)}\left(A_{i} f^{j /(20 h)} u\right)\right. \\
& \left.+\|Q u\|^{(n)}+\|u\|_{(4)}^{(n-1 /(20 h))}\right) .
\end{aligned}
$$

From Lemma 6.39-(2), and (6.24) there exist $B_{i} \in O p^{f} \sum_{1 / 2}^{2(0,-1,1)}$ for $i=1, \cdots, s$ such that

$$
O p\left(p_{3}(t, x, 1, \zeta(t, x))^{-1} d_{l}(f \xi)^{3}\right) \cdot A_{i}-B_{i} \in \mathcal{C}_{0} .
$$


On the other hand, since we can write $O p\left({ }^{f} L_{l}(f \xi)^{2}\right)=\left(1-\mu_{l} \zeta\right) f^{2} D_{x}^{2} \cdot \tilde{L}_{l}+$ $\sum_{k=1}^{2} a_{k} f^{k} D_{x}^{k}$ with $a_{k} \in B_{h, T_{0}}$, it follows from (3.19), Lemmas 6.40-(2), 6.39-(2), and (6.24) that there exist $B_{i}^{\prime} \in O p{ }^{f} \sum_{1 / 2}^{(0,-1,1)}, i=1, \cdots, s$ such that

$$
O p\left(p_{3}(t, x, 1, \zeta(t, x))^{-1} d_{l} A_{1 l}{ }^{f} L_{l}(f \xi)^{2}\right) \cdot A_{i}-B_{i}^{\prime} \tilde{L}_{l} \in \mathcal{C}_{0}
$$

Thus, since

$$
\begin{aligned}
& p_{3}(t, x, 1, \zeta(t, x))^{-1}{ }^{f} q_{3}={ }^{f} q^{(l)}+p_{3}(t, x, 1, \zeta(t, x))^{-1}\left(d_{l}(f \xi)^{3}\right. \\
& \left.\quad+d_{l} A_{1 l}{ }^{f} L_{l}(f \xi)^{2}\right),
\end{aligned}
$$

it follows from (8.26), (8.27), Lemmas 7.3-(1), 7.2-(3), 7.4-(1), 4.21-(1), 4.18(i) that for small $T$ and $n \geq m+1$,

$$
\begin{aligned}
& M_{0 l, n-j /(20 h)}\left(A_{i} u\right) \leq C_{2}\left\|O p{ }^{f} q^{(l)} A_{i} u\right\|^{(n-j /(20 h))} \\
& \quad \leq C_{3}\left(\left\|p_{3}(t, x, 1, \zeta(t, x))^{-1} O p{ }^{f} q_{3} u\right\|^{(n-j /(20 h))}\right. \\
& \quad+n^{-1 / 2} M_{0, n-j /(20 h)}(u)+\|u\|_{(3)}^{(u-(j+1) /(20 h))}+\left\|B_{i} u\right\|^{(n-1 /(20 h))} \\
& \left.\quad+\left\|B_{i}^{\prime}\left(\tilde{L}_{l}-D_{t}\right) u\right\|^{(n-j /(20 h))}+\left\|B_{i}^{\prime} D_{t} u\right\|^{(n-j /(20 h))}\right) \\
& \quad \leq C_{4}\left(\|Q u\|^{(n-j /(20 h))}+\|u\|_{(4)}^{(n-(j+1) /(20 h))}+\|u\|_{(4, n-j /(20 h))}\right) .
\end{aligned}
$$

Combining this and (8.25) and using Lemmas 4.21-(2), 7.8, (4.55), and Lemma 7.1-(6) we have that for small $T, n \geq m+1$,

$$
\begin{aligned}
& M_{0 l, n}(A u) \leq C_{5}\left\{\sum _ { j = 0 } ^ { m - 1 } n ^ { - ( m - j - 1 ) / 2 } \left(\left\|Q f^{j /(20 h)} u\right\|^{(n-j /(20 h))}\right.\right. \\
& \left.\quad+\left\|f^{j /(20 h)} u\right\|_{(4)}^{(n-1(j+1) /(20 h))}+\left\|f^{j /(20 h)} u\right\|_{(4, n-j /(20 h))}\right) \\
& \left.\quad+\|Q u\|^{(n)}+\|u\|_{(4)}^{(n-1 /(20 h))}\right\} \\
& \quad \leq C_{6}\left(\sum_{j=0}^{m-1} n^{-(m-j-1) / 2}\left\|f^{j /(20 h)} u\right\|_{(4, n-j /(20 h))}+\|Q u\|^{(n)}+\|u\|_{(4)}^{(n-1 /(20 h))}\right) \\
& \quad \leq C_{7}\left(\left\|f^{(m-1) /(20 h)} u\right\|_{(4, n-(m-1) /(20 h))}+\|Q u\|^{(n)}+\|u\|_{\left(\begin{array}{l}
(n-1 /(20 h)) \\
(n)
\end{array}\right) .}\right.
\end{aligned}
$$

This completes the proof of Lemma 8.4.

Remark 8.5. In the proof of Lemmas 8.3 and 8.4 we do not use (1) of (3.13). So these two lemmas hold in all cases of (3.13).

Taking $N=2 N_{0}+1$ in (8.5) and estimating the first (resp. second) summation by Lemma 8.2 (resp. 8.5) we obtain that for small $T$ and $n \geq 2 N_{0}+2$,

$$
\begin{aligned}
\|u\|_{(6)}^{(n)} \leq & C_{4}\left(\|Q u\|^{(n)}+\|u\|_{(4)}^{(n-1 /(20 h))}+\left\|f^{r_{0}} u\right\|_{\left(4, n-r_{0}-3\right)}\right. \\
& \left.+\left\|f^{N_{0} /(10 h)} u\right\|_{\left(4, n-N_{0} /(10 h)\right)}\right) .
\end{aligned}
$$

Since $N_{0} /(10 h)=2\left(2 r_{0}+4\right)>r_{0}+3$, the last term on the right can be dominated 
by $\left.\left\|f^{\prime \prime} 0 u\right\|_{\left(4, n-r_{0}-3\right.}\right)$ in view of Lemma 4.21-(4) and (4.55). So combining this inequality and (8.4), and using (4.55) we get that for small $T$ and $n \geq N_{0}+3$,

$$
\|u\|_{(6)}^{(n)}+\left\|f^{r} u\right\|_{\left(4, n-r_{0}\right)} \leq C_{5}\|Q u\|^{(n)} .
$$

Taking $N=2 N_{0}+2$ in the inequality of Lemma 7.9 we see by the similar argument that for small $T$ and $n \geq 2 N_{0}+3$,

$$
\begin{aligned}
& \sum_{1 \leq|\mu|+\mid \nu^{\prime \leq 2}}\left\|Q_{\mu_{\nu}} u\right\|^{(n)} \leq C_{6}\left(\|Q u\|^{(n-1 /(20 h))}+n^{-1 / 2}\|Q u\|^{(n)}\right. \\
& \left.\quad+\|u\|_{(4)}^{(n-1 /(20 h))}+\left\|f^{\prime} 0 u\right\|_{\left(4, n-r_{0}-3\right)}\right) .
\end{aligned}
$$

Applying (8.29) to the latter two terms on the right we get the second desired inequality. The proof is complete.

Next we consider the case (2).

Proposition 8.6. Assume that (2) of (3.13) holds. Then there exists $r_{0}$, $n_{0} \in \boldsymbol{N}$ with $n_{0} \geq r_{0}+1$ such that for small $T$ and $n \geq n_{0}$,

$\left\|f^{r_{0}} u\right\|_{\left(4, n-r_{0}\right)}+\|u\|_{(6)}^{(n)} \leq C\|Q u\|^{(n)}$,

$\left\|Q_{\mu_{\vartheta}} u\right\|^{(n)} \leq C\left(\|Q u\|^{(n-1 /(20 h))}+n^{-1 / 2}\|Q u\|^{(n)}\right)$ when $1 \leq|\mu|+|\nu| \leq 2$.

Proof. As in the proof of Proposition 8.1 we may assume $Q=p_{3}(t, x, 1, \zeta$ $(t, x))^{-1} O p{ }^{f} q$. Set $\Phi_{i N}=O p{ }^{f} \varphi_{i N}, \Phi_{0 l N}=O p{ }^{f} \varphi_{0 l N}$ for $N \geq 2$. Then using Corollary 6.27, Lemma 4.18-(4) and (5), Lemmas 4.21, 7.2-(3), and the fact that $\sum_{i=0}^{2} A_{i} D_{t}^{i} f^{3 / 2} A \in \mathcal{C}_{0}$ for $A \in O p \Sigma^{(0, M, 0)}, A_{i} \in O p{ }^{f} \Sigma^{M^{\prime}(0,-2,0)}, i=0,1,2$ with $M, M^{\prime} \geq 0$ and $M^{\prime}-2^{-1} M \leq-1$ which can be shown by using Fact 4.6-7, (4.3), (4.6), and Corollary 4.12-(iv) as in the proof of Corollary 4.12-(i), it is not hard to see that for $r \in \mathbb{N}$ we have that

$$
\begin{aligned}
& \left\|f^{r} u\right\|_{(4, n-r)} \leq C_{1}\left(n^{-1 / 2}\|Q u\|^{(n-r-2 \kappa / 3)}\right. \\
& \left.\quad+\|u\|_{(3)}^{(n-2 \kappa / 3-r-1 /(20 h))}+\sum_{i \in I}\left\|f^{r} \Phi_{i N}{ }^{N} u\right\|_{(4, n-r)}+\sum_{i=1}^{2}\left\|f^{r} \Phi_{0 l N}{ }^{N} u\right\|_{(4, n-r)}\right) \\
& \quad \text { for small } T \text { and } n \geq 2 \kappa / 3+r+2
\end{aligned}
$$

In the same way as in the proof of Proposition 8.1 we have from Corollary 6.27, Lemma 4.21, (4.55),

$$
\begin{aligned}
& \|u\|\left(\begin{array}{l}
n \\
6
\end{array}\right) \leq C_{2}\left(\sum_{i \in I}\left\|\Phi_{i N}{ }^{N} u\right\|\left\|_{\left(\begin{array}{l}
n \\
6
\end{array}\right)+\sum_{l=1}^{2}}\right\| \Phi_{0 l N}{ }^{N} u\|\|_{\left(\begin{array}{l}
n \\
6
\end{array}\right)}\right) \\
& \text { for small } T \text { and } n \geq 2 \kappa / 3+1 .
\end{aligned}
$$

In case (2) of (3.13) we have

$$
c \in E_{h, T_{0}} \backslash\{0\}
$$


So setting, keeping (7.11) and a remark just after it in mind, that

$$
\begin{aligned}
\tilde{\tilde{L}}_{l} & :=\tilde{L}_{l}-\left(1-\mu_{l} \zeta\right)^{-1} c_{l} f D_{x} \\
& =\tilde{L}_{l}-\left(1+A_{0 l}\right)^{-1}(-1)^{l+1}\left(1-\mu_{l} \zeta\right)^{-1} c b_{l} f D_{x},
\end{aligned}
$$

and using Lemma 6.10-(2), and Lemmas 4.21, 7.1-(8), one can show that there exists $r_{1} \in N$ such that for any $r \geq r_{1}, r \in N$ we have that $t^{r} f^{r} D_{t}$ and $t^{r} f^{r+1} D_{x}$ can be expressed as a linear combinations of $\tilde{L}_{l}$ and $\tilde{\tilde{L}}_{l}$ with coefficients in $B_{h, T_{0}}$, and that

$$
\begin{aligned}
& \left\|f^{r} \Phi_{i N}{ }^{N} u\right\|_{(4, n-r)} \leq C_{3}\left(\left\|f^{r-r_{1}} \tilde{L}_{l(i)} \Phi_{i N}{ }^{N} u\right\|_{\left(2, n-r+r_{1}\right)}\right. \\
& \left.\quad+\left\|f^{r-r_{1}} \tilde{\tilde{L}}_{l(i)} \Phi_{i N}{ }^{N} u\right\|_{\left(2, n-r+r_{1}\right)}+n^{-1 / 2}\|u\|_{(2)}^{(2-1)}\right), \\
& \left\|f^{r} \Phi_{0 l N}{ }^{N} u\right\|_{(4-r)} \leq C_{3}\left(\left\|f^{r-r_{1}} \tilde{L}_{l} \Phi_{0 l N}{ }^{N} u\right\|_{\left(2, n-r+r_{1}\right)}\right. \\
& \left.\quad+\left\|f^{r-r_{1}} \tilde{\tilde{L}}_{l} \Phi_{0 l N}{ }^{N} u\right\|_{\left(2, n-r+r_{1}\right)}+n^{-1 / 2}\|u\|_{(2)}^{(n-1)}\right),
\end{aligned}
$$

for $n \geq r+1$, where $l(\mathrm{i}), i \in I$ is as in Lemma 3.2. (3.26) and the fact that $c \in E_{h, T_{0}}$ shows

$$
c \xi \in \Sigma_{\operatorname{supp} \varphi_{i}}^{2 / 3(0,-2,0)} \text { for } i \in I_{1} \text {. }
$$

So from (8.33), Lemmas 6.30, 6.31-(1), and (6.42)

$$
\tilde{\tilde{L}}_{l(i)} \Phi_{i N}-\tilde{L}_{l(i)} \Phi_{i N} \in O p^{f} \Sigma^{2 / 3(0,-2,0)}
$$

Using (8.34), (8.36), Lemmas $4.18,4.21$, and the inequality that

$$
\begin{aligned}
& \left\|\Lambda_{\alpha} u\right\|_{(s, n)} \leq C\|u\|_{(s+[2 \alpha]+1, n)} \\
& \text { for } s \in N \text { with } 0 \leq s \leq 5 \text { and } \alpha>0 \text { with } s+2 \alpha \leq 6,2 \alpha \notin N,
\end{aligned}
$$

we have for any $r \in \boldsymbol{N}$ with $r \geq r_{1}$ that for $n \geq r+1$ and $i \in I_{1}$,

$$
\begin{aligned}
& \left\|f^{r} \Phi_{i N}{ }^{N} u\right\|_{(4, n-r)} \leq C_{4}\left(\left\|f^{r-r_{1}} \tilde{L}_{l(i)} \Phi_{i N}{ }^{N} u\right\|_{\left(2, n-r+r_{1}\right)}\right. \\
& \left.\quad+\left\|\Lambda_{2 / 3} f^{r-r_{1}} \Phi_{i N}{ }^{N-1} u\right\|_{\left(2, n-r+r_{1}\right)}+\|u\|_{(3)}^{(n-1)}\right) .
\end{aligned}
$$

Similarly using the fact that by (7.11) and (3.23),

$$
\tilde{\tilde{L}}_{l(1)}-L_{i 1} \in O p^{f} \sum_{1 / 2}^{2 / 3(0,-1,1)},
$$

we have for any $r \in \boldsymbol{N}$ with $r \geq r_{1}$ that for $n \geq r+1$ and $i \in I_{2}$,

$$
\begin{aligned}
& \left\|f^{r} \Phi_{i N}{ }^{N} u\right\|_{(4, n-r)} \leq C_{5}\left(\left\|f^{r-r_{1}} \tilde{L}_{l(i)} \Phi_{i N}{ }^{N} u\right\|_{\left(2, n-r+r_{1}\right)}\right. \\
& \left.\quad+\left\|f^{r-r_{1}} L_{i 1} \Phi_{i N}{ }^{N} u\right\|_{\left(2, n-r+r_{1}\right)}+\|u\|_{(3)}^{(n-1)}\right) .
\end{aligned}
$$

Noting (7.11) and applying the similar argument as above to the first terms on the right of (8.38) and (8.40) we obtain for any $r \in \boldsymbol{N}$ with $r \geq r_{1}$ that for $n \geq r+1$ 
and $i \in I$,

$$
\begin{aligned}
& \left\|f^{r} \Phi_{i N}{ }^{N} u\right\|_{(4, n-r)} \leq C_{6}\left(\sum_{j=1}^{2}\left\|f^{r-r_{1}} L_{i j} \Phi_{i N}{ }^{N} u\right\|_{\left(2, n-r+r_{3}\right)}\right. \\
& \left.\quad+\left\|\Lambda_{2 / 3} f^{r-r_{1}} \Phi_{i N}{ }^{N-1} u\right\|_{\left(2, n-r+r_{1}\right)}+\|u\|_{(3)}^{(n-1)}\right) .
\end{aligned}
$$

Since by using Lemmas 7.1-(8), 4.12-(1), and Cor. 4.3-(ii) the second term on the right of (8.41) is dominated by $\left\|\Lambda_{2 / 3} D_{t} f^{r-r_{1}} \Phi_{i N}{ }^{N-1} u\right\|^{\left(n-r+r_{1}\right)}+\| \Lambda_{2 / 3} f^{r-r_{1}+1}$ $D_{x} \Phi_{i N}{ }^{N-1} u\left\|^{\left(n-r+r_{1}\right)}+n^{-1 / 2}\right\| u \|_{(2)}^{(n-2)}$, repeating the similar argument as above once again we get for any $r \in N$ with $r \geq 2 r_{1}$ that for $n \geq r+1$ and $i \in I$,

$$
\begin{aligned}
& \left\|f^{r} \Phi_{i N}{ }^{N} u\right\|_{(4, n-r)} \leq C_{7}\left(\sum_{j=1}^{2}\left\|L_{i j} \Phi_{i N}{ }^{N} u\right\|_{\left(2, n-r+r_{1}\right)}\right. \\
& \left.\quad+\left\|\Lambda_{2 / 3} L_{i j} \Phi_{i N}{ }^{N-1} u\right\|^{\left(n-r+2 r_{1}\right)}+\|u\|_{(3)}^{\left(n-r+2 r_{1}-1\right)}\right) .
\end{aligned}
$$

On the other hand, using the fact that from (3.19) and Lemma 6.39-(2),

$$
d_{l} f D_{x} \cdot O p \varphi \in t^{-k} \mathcal{A}^{0,3} \text { for } \varphi \in \Sigma_{\varphi_{01}}^{0},
$$

in the similar way as above we have for $r \in N, r \geq r_{1}$ that for $n \geq r+1$

$$
\begin{aligned}
& \left\|f^{r} \Phi_{0 l N}{ }^{N} u\right\|_{(4, n-r)} \leq C_{8}\left(\left\|f^{r-r_{1}} \tilde{L}_{l} \Phi_{0 l N}{ }^{N} u\right\|_{\left(2, n-r+r_{1}\right)}\right. \\
& \left.\quad+\left\|f^{r-r_{1}} L_{l}^{\prime} \Phi_{0 l N}{ }^{N} u\right\|_{\left(2, n-r+r_{1}\right)}+n^{-1 / 2}\|u\|_{(2)}^{\left(n-r+r_{1}+\kappa-2\right)}\right) .
\end{aligned}
$$

Therefore, taking

$$
r=r_{0}:=r_{1}+\max \left(r_{1}, \kappa\right)+1,
$$

and Lemma 7.1-(1) and Corollary 5.2 we obtain from (8.42) and (8.44) that

$$
\begin{aligned}
& \left\|f^{r_{0}} u\right\|_{\left(4, n-r_{0}\right)} \leq C_{9}\left\{n^{-1 / 2}\|Q u\|^{\left(n-r_{0}-1\right)}+\|u\|_{(3)}^{(n-2)}\right. \\
& \quad+\sum_{i \in I}\left(M_{i, n-3}\left(\Phi_{i N}{ }^{N} u\right)+M_{i, n-3}\left(\Phi_{i N}{ }^{N-1} u\right)\right)+\sum_{l=1}^{2}\left(M_{0 l, n-3}\left(\Phi_{0 l N}{ }^{N} u\right)\right. \\
& \left.\left.\quad+M_{0 l, n-3}\left(\Phi_{0 l N}{ }^{N-1} u\right)\right)\right\} \\
& \quad \text { for small } T \text { and } n \geq r_{0}+3 .
\end{aligned}
$$

Estimating the right of (8.32) by Lemma 7.1-(3) we have for small $T$ and $n \geq 2$,

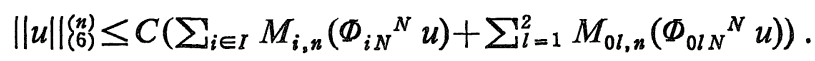

We set

$$
N_{0}:=20 h\left(2 r_{0}+1\right) \text {. }
$$

Lemma 8.7. Let $N_{1}, N_{2} \in N$ with $N_{2} \geq N_{0}$ and $N_{1}=2 N_{2}+3$. Let $A \in \Sigma_{N_{1}, \varphi_{i}}$, $A_{2} \in \Sigma_{N_{1}, \varphi_{i}}$ for $i \in I$. Then for small $T$ and $n \geq N_{2}+3$, 


$$
\sum_{j=1}^{2} M_{i, n}\left(A_{j} u\right) \leq C\left(\|Q u\|^{(n)}+\|u\|_{(4)}^{(n-1 /(20 h))}+\left\|f^{r_{0}+r_{1}} u\right\|_{\left(4, n-r_{0}-r_{1}\right)}\right) .
$$

Proof. Using Corollary 7.7 with $k=N_{2}$ and $k^{\prime}=3$ to $M_{i, n}\left(A_{1} u\right)$, and next estimating the terms of the form $n^{-\left(N_{2}-r\right) / 2} M_{i, n-1 /(20 h)}\left(A f^{r /(20 h)} u\right), A \in \Sigma_{3, \varphi_{i}}$, $0 \leq r \leq N_{2}$ on the ritht of the resulting inequality by using (7.3), (7.6), Lemmas 7.8, 4.21-(1), 7.1-(6) we get for some $B_{1}, \cdots, B_{s} \in \Sigma_{2, \varphi_{i}}$ that for small $T$ and $n \geq N_{2}+3$,

$$
\begin{aligned}
& M_{i, n}\left(A_{1} u\right) \leq C_{1}\left(\|Q u\|^{(n)}\right. \\
& \quad+\sum_{r=0}^{N_{2}+1} \sum_{j=1}^{s} n^{-\left(N_{2}+1-r\right) / 2} M_{i, n-r /(20 h)}\left(B_{j} f^{r /(20 h)} u\right) \\
& \left.\quad+\left\|f^{N_{2} /(20 h)} u\right\|_{\left(4, n-N_{2} /(20 h)\right)}+\|u\|_{(4)}^{(n-1 /(20 h))}\right) .
\end{aligned}
$$

Using Corollary 7.7 with $k=N_{2}$ and $k^{\prime}=2$ we have for some $B_{s+1}, \cdots, B_{s+s^{\prime}} \in \Sigma_{2, \varphi_{i}}$ that for small $T$ and $n \geq N_{2}+3$,

$$
\begin{aligned}
& M_{i, n}\left(A_{2} u\right) \leq C_{2}\left(\|Q u\|^{(n)}\right. \\
& \quad+\sum_{r=0}^{N_{2}} \sum_{j=s+1}^{s+s^{\prime}} n^{-\left(N_{2}-r\right) / 2} M_{i, n-r /(20 h)}\left(B_{j} f^{r /(20 h)} u\right) \\
& \left.\quad+\left\|f^{N_{2} /(20 h)} u\right\|_{\left(4, n-N_{2} /(20 h)\right)}+\|u\|_{(4)}^{(n-1 /(20 h))}\right)
\end{aligned}
$$

Let us estimate terms involving $B_{j}$ in (8.49) and (8.50). To do so we let $\Phi \in \Sigma_{2, \varphi_{i}}$ and we write $\Phi=\Phi_{1} \Phi_{2}, \Phi_{j} \in \Sigma_{1, \varphi_{i}}$. From Lemma 6.6,Op $\mu . \Phi_{1}-\Phi_{1} Q$ $\in \mathcal{C}+\mathcal{C}_{0}$ with $L_{j}=L_{i j}$ and $\pi$ there. So using (3.23) and the last fact of (3.26) we have

$$
\begin{aligned}
& \text { Op } \pi \cdot \Phi_{1}-\Phi Q-\sum_{k=0}^{2} \tilde{A_{k}} \tilde{L}_{I(i)}{ }^{k} \in \mathcal{C}_{0} \\
& \text { with } \tilde{A_{2}} \in \mathcal{A}^{1 / 2,1 / 2,3}, \tilde{A_{1}} \in \mathcal{A}^{3 / 2,1 / 2,3}, \\
& \tilde{A_{0}} \in \mathcal{A}^{3 / 2,3 / 2,3}+\mathcal{A}^{2,1,3}+t^{-1+1 /(6 h)} \mathcal{A}^{3 / 2,1 / 2,3} .
\end{aligned}
$$

Then for any $\alpha \geq 0$ we have that for small $T$ and $n \geq 3$,

$$
\begin{aligned}
& M_{i, n}\left(\Phi f^{\infty} u\right) \leq C_{1}^{\prime}\left\|O p \pi \cdot \Phi f^{\alpha} u\right\|^{(n)} \quad(\text { Lemma 7.3-(1)) } \\
& \quad \leq C_{2}^{\prime}\left(\left\|Q \Phi_{2} f^{\infty} u\right\|^{(n)}+\sum_{k=0}^{2}\left\|\tilde{A}_{k} \tilde{L}_{l(i)} \Phi_{2} f^{\alpha} u\right\|^{(n)}+n^{-1 / 2}\|Q u\|^{(n)}\right. \\
& \left.\quad+\|u\|_{(4)}^{(n-1 /(20 h))}\right) \quad((8.51), \text { Lemmas } 6.13,7.2-(3), 7.4-(1), 4.21-(2)) \\
& \quad \leq C_{3}^{\prime}\left(\|Q u\|^{(n)}+M_{0 l(i), n}\left(\Phi_{2} f^{\alpha} u\right)+\left\|f^{\alpha} u\right\|_{(4, n+1-1 /(20 h)}+\|u\|_{(4)}^{(n-1 /(20 h))}\right. \\
& \left.\quad+\left\|\Lambda_{3 / 2} \tilde{L}_{l(i)} \Phi_{2} f^{\alpha} u\right\|^{(n+1 / 2(1-1) /(10 h))}\right) \quad((7.6), \text { Lemmas } \\
& \\
& \quad 7.8,4.18-(1), 4.21-(1) \text { and }(2)) .
\end{aligned}
$$

By Corollary 4.3 we have that for small $T$ and $n \geq 2$,

$$
\begin{aligned}
\left\|\Lambda_{3 / 2} \tilde{L}_{l(i)} \Phi_{2} f^{\alpha} u\right\|^{(n)} \leq & C_{8}^{\prime}\left(n^{-1 / 2} M_{0 l(i), n-1}\left(\Phi_{2} f^{\alpha} u\right)\right. \\
& \left.+\left\|\Lambda_{1 / 2} f D_{x} \tilde{L}_{l(i)} \Phi_{2} f^{\alpha} u\right\|^{(n)}\right) .
\end{aligned}
$$


It is not hard to check using Lemma 4.18-(4) that for any $r \in \mathbb{N}$,

$$
\begin{aligned}
& f D_{x} \tilde{L}_{l(i)}\left[\Phi_{2}, f^{r}\right]=f^{r} \tilde{B}_{1} D_{t}+f^{r} \tilde{B}_{2} \\
& \text { with } \tilde{B}_{1} \in \mathcal{A}^{1 / 2,3}, \tilde{B}_{2} \in \mathcal{A}^{3 / 2,3}+\mathcal{A}^{1,1 / 2,3} .
\end{aligned}
$$

From (3.26) and Lemma 6.39-(2)

$$
\left(d_{b(i)} f \xi\right) \circ \varphi \in^{f} \Sigma^{0} \text { for } \varphi \in \Sigma_{\varphi_{i}} .
$$

Using (8.54), (8.55), (7.8), and noting the manner of choice of $r_{1}$ (cf. (8.34)), one can show in a straightforward way by taking care of commutation that for $n \geq r_{1}+1$

$$
\begin{aligned}
& \left\|\Lambda_{1 / 2} f D_{x} \tilde{L}_{l(i)} \Phi_{2} f^{r_{1}} u\right\|^{\left(n-r_{1}\right)} \leq C_{5}^{\prime}\left(\|u\|_{\left(4, n-r_{1}\right)}+\|u\|_{(3)}^{(n-1)}\right. \\
& \left.\quad+\left\|\tilde{L}_{l(i)}{ }^{2} \Phi_{2} u\right\|_{(1, n)}+\left\|\tilde{L}_{l(i)} L_{l(i)}^{\prime} \Phi_{2} u\right\|_{(1, n)}\right) .
\end{aligned}
$$

From (8.52), (8.53), (8.56), (4.55), Lemmas 7.1-(4), 4.21-(2) and (4) we have for any $r \in N$ with $r \geq N_{2}-2$ which implies $r /(20 h) \geq 2 r_{0}+1$,

$$
\begin{aligned}
& M_{i, n-r /(20 h)}\left(\Phi f^{r /(20 h)} u\right) \leq C_{6}^{\prime}\left(\|Q u\|^{(n-1 /(20 h))}\right. \\
& \quad+\|u\|_{(4)}^{\left.\left(n-(r+1) /(20 h)+r_{1}\right)\right)}+M_{0 l(i), n-r /(20 h)}\left(\Phi_{2} f^{r /(20 h)} u\right) \\
& \quad+M_{0 l(i), n-r /(20 h)+r_{1}}\left(\Phi_{2} f^{r /(20 h)-r_{1}} u\right) \\
& \left.\quad+\left\|f^{r /(20 h)-r_{1}} u\right\|_{\left(4, n+r_{1}-(r+1) /(20 h)+1 / 2\right)}\right) .
\end{aligned}
$$

From (8.52), (4.55), Lemmas 7.1-(4) and (6), 4.21-(2) we have for any $r \in \mathbb{N}$ with $0 \leq r \leq N_{2}-3$ that for small $T$ and $n \geq N_{2}+3$,

$$
\begin{gathered}
n^{-\left(N_{2}-r\right) / 2} M_{i, n-r /(20 h)}\left(\Phi f^{r /(20 h)} u\right) \leq C_{7}^{\prime}\left(n^{-3 / 2}\|Q u\|^{(n)}\right. \\
\left.+n^{-1}\|u\|_{(4)}^{(n-1 /(20 h))}+n^{-1} M_{0 l(i), n}\left(\Phi_{2} f^{r /(20 h)} u\right)\right) .
\end{gathered}
$$

Now from (3.26) and Lemma 6.39-(2) for $\varphi \in \Sigma_{\varphi_{i}}^{0}$,

$$
\begin{aligned}
& \left(d_{l(i)}(f \xi)^{3}+d_{l(i)}(f \xi)^{2} A_{1 l(i)}{ }^{f} L_{l(i)}\right) \circ \varphi=b_{1} \tau+b_{2} \\
& \quad \text { with some } b_{l} \in{ }^{f} \Sigma^{(0,-2,0)}, b_{2} \in{ }^{f} \Sigma^{(0,4,0)}+f \Sigma^{(-1,-2,0)} .
\end{aligned}
$$

So from (8.59) we have for any $A \in \Sigma_{1, \varphi_{i}}$ that for small $T$ and $n \geq 2$.

$$
\begin{aligned}
& M_{0 l(i), n}(A u) \leq C\left(M_{0, n}(A u)+\|u\|_{(4, n)}+\|u\|_{(3)}^{(n-1)}\right) \\
& \quad(\text { by Lemmas 7.3-(1), 4.21-(1)) } \\
& \quad \leq C^{\prime}\left(\|Q u\|^{(n)}+\|u\|_{(4, n)}+\|u\|_{(3)}^{(n-1 /(20 h))}\right)
\end{aligned}
$$

(by Lemma 7.4-(2)).

Substituting $f^{\alpha} u, \alpha \geq 0$ for $u$ in (8.60) and using Lemmas 7.8, 4.21 we have for any $A \in \Sigma_{1_{0} \varphi_{i}}, \alpha \geq 0$ that for small $T$ and $n \geq 2$, 


$$
M_{0 l(i), n}\left(A f^{\alpha} u\right) \leq C\left(\|Q u\|^{(n)}+\left\|f^{\alpha} u\right\|_{(4, n)}+\|u\|_{(4)}^{(n-1 /(20 h))}\right) .
$$

Applying this inequality to (8.57) and (8.58) we get using Lemmas 4.21-(4) and 7.1-(6) that when $r \geq N_{2}-2$, for small $T$ and $n \geq N_{2}+3$,

$$
\begin{aligned}
M_{i, n-r /(20 h)}\left(\Phi f^{r /(20 h)} u\right) \leq & C_{8}^{\prime}\left(\|Q u\|^{(n-r /(20 h))}+\|u\|_{(4)}^{\left(n-r_{0}-r_{1}\right)}\right. \\
& \left.+\left\|f^{r_{0}+r_{1}} u\right\|_{\left(4, n-r_{0}-r_{1}\right)}\right),
\end{aligned}
$$

and that when $0 \leq r \leq N_{2}-3$, for small $T$ and $n \geq N_{2}+3$,

$$
\begin{aligned}
n^{-\left(N_{2}-r\right) / 2} M_{i, n-r /(20 h)}\left(\Phi f^{r /(20 h)} u\right) \leq & C_{9}^{\prime}\left(n^{-1}\|Q u\|^{(n)}\right. \\
& \left.+n^{-1 / 2}\|u\|_{(4)}^{(n-1 /(20 h))}\right)
\end{aligned}
$$

Applying (8.61) and (8.62) to the terms involving $B_{j}$ in (8.49) and (8.50), noting $N_{2} \geq 2 r_{0}+1$, and using Lemma 4.21-(4) and (4.55) we get the desired inequality. The proof of Lemma 8.7 is complete.

We set

$$
N_{1}=2 N_{0}+3
$$

Then by Lemma 8.7 we have that for $i \in I$, small $T$, and $n \geq N_{0}+3$,

$$
\begin{aligned}
M_{i, n}\left(\Phi_{i N_{1}}{ }^{N_{1}} u\right)+M_{i n}\left(\Phi_{i N_{1}}{ }^{N_{1}-1} u\right) \leq & C_{11}\left(\|Q u\|^{(n)}+\|u\|_{(4)}^{(n-1 /(20 h))}\right. \\
& \left.+\left\|f^{r_{0}+r_{1}} u\right\|_{\left(4, n-r_{0}-r_{1}\right)}\right)
\end{aligned}
$$

By Remark 8.5 we can use Lemma 8.4 to conclude that for any $l$, small $T$, and $n \geq N_{1}+1$

$$
\begin{aligned}
& M_{0 l, n}\left(\Phi_{0 l N_{1}}^{N_{1}} u\right)+M_{0 l, n}\left(\Phi_{0 l N_{1}}^{N_{1}-1} u\right) \leq C_{12}\left(\left.\|Q u\|\right|^{(n)}\right. \\
& +\|u\|_{(4)}^{(n-1 /(20 h))}+\left\|f^{\left(N_{1}-1\right) /(20 h)} u\right\|_{\left.\left(4, n-\left(N_{1}-1\right) / 20 h\right)\right)} \\
& \left.+\left\|f^{\left(N_{1}-2\right) /(20 h)} u\right\|_{\left(4, n-\left(N_{1}-2\right) /(20 h)\right)}\right) \text {. }
\end{aligned}
$$

Since $\left(N_{1}-2\right) /(20 h) \geq 2\left(2 r_{0}+1\right)$, the rest of proof of Proposition 8.6 is now straightforward by using (8.46), (8.47), (8.64), (8.65), and by taking $N=N_{1}$ in Lemma 7.9 as in the proof of Proposition 8.1 .

Q.E.D.

Finally we consider the case (3).

Proposition 8.8. Assume that (3) of (3.13) holds. Set for $s=1,2, n \geq 1$, and $u \in \mathcal{S}_{T}$,

$$
|| u\left\|^{(s, n)}=\sum_{k=0}^{s} n^{k / 2}\right\| \Lambda_{(s-k) / 2} u \|^{(n+(s+k) / 2)} .
$$

Then there exists $n_{0} \in \boldsymbol{N}$ such that for small $T$ and $n \geq n_{0}$, 


$$
\begin{aligned}
\|u\|_{(6)}^{(n)} & +\sum_{k=1}^{2}\left\|\tilde{L}^{k} u\right\|^{(3-k, n)} \leq C\|Q u\|^{(n)}, \\
& \sum_{1 \leq \mid \mu_{1+|\nu| \leq 2}}\left\|Q_{\mu_{y}} u\right\|^{(n)} \leq C\left(\|Q u\|^{(n-1 /(20 h))}+n^{-1 / 2}\|Q u\|^{(n)}\right) .
\end{aligned}
$$

Proof. Let $\varphi_{i}^{*} \in \Sigma_{0}^{0}, i \in I$ be in Lemma 3.2 and define for $s, j, k \in\{1,2,3\}$ with $s \neq j$,

$$
b_{s j k}=\left[\begin{array}{cl}
\sigma_{i k} /\left(\sigma_{i s}-\sigma_{i j}\right) \varphi_{i}^{*} & \text { on supp } \varphi_{i}^{*} \\
0 & \text { on }\left(\operatorname{supp} \varphi_{i}^{*}\right)^{c}
\end{array}\right.
$$

Then $b_{i j k}$ satisfies with the notations in Definitions 3.1 and 4.4

$$
\left|\partial^{\alpha} b_{s j k}\right| \leq C_{\alpha} \tilde{\psi}_{1}^{-\alpha_{1}} \cdot \psi_{2}^{-\alpha_{2}} \cdot \psi_{3,1 / 6}^{-\alpha_{3}} .
$$

Proof of (8.66). It suffices to consider this on supp $\varphi_{i}^{*}$. First from (3.28),

$$
\left|b_{s j k}\right| \leq C \text {. }
$$

Next from (3.22)' and (3.28) we have that for $\alpha$ with $\alpha_{1}>0$

$$
\begin{aligned}
\left|\partial^{\alpha} \sigma_{i k}\right| & \leq C_{\alpha} t^{-1+1 /(6 h)} \tilde{\psi}_{1}^{-\alpha_{1}+1} \psi_{2}^{-\alpha_{2} \cdot \psi_{3,0}^{1-\alpha_{3}}} \\
& \leq C_{\alpha}^{\prime} t^{-1+1 /(6 h)+\kappa / 3}\langle\xi\rangle^{1 / 2} \tilde{\psi}_{1}^{-\alpha_{1}+1} \psi_{2}^{-\alpha_{2}} \cdot \psi_{3,0}^{-\alpha_{3}} \cdot\left|\sigma_{i s}-\sigma_{i j}\right| \\
& \leq C_{\alpha}^{\prime} \tilde{\psi}_{1}^{-\alpha_{1}} \psi_{2}^{-\alpha_{2} \cdot} \psi_{3,0}^{-\alpha_{3}}\left|\sigma_{i s}-\sigma_{i j}\right|,
\end{aligned}
$$

that for $\alpha$ with $\alpha_{1}=0$ and $\alpha_{2}>0$,

$$
\begin{aligned}
& \left|\partial^{\alpha} \sigma_{i k}\right| \leq C_{\alpha} t^{1 /(6 h)} \psi_{2}^{-\alpha_{2}+1} \cdot \psi_{3,0}^{1-\alpha_{3}} \leq C_{\alpha}^{\prime} t^{1 /((6 h)+\kappa / 3}\langle\xi\rangle^{1 / 2} \\
& \quad \times \psi_{2}^{-\alpha_{2}+1} \psi_{3,0}^{-\alpha_{3}} \cdot\left|\sigma_{i s}-\sigma_{i j}\right| \leq C_{\alpha}^{\prime} \cdot \psi_{2}^{-\alpha_{2}} \cdot \psi_{3,0}{ }^{-\alpha_{3}} \cdot\left|\sigma_{i s}-\sigma_{i j}\right|,
\end{aligned}
$$

and that for $\alpha$ with $\alpha_{3}>0$ and $\alpha_{1}=\alpha_{2}=0$,

$$
\begin{aligned}
\left|\partial^{\alpha} \sigma_{i k}\right| & \leq C_{\alpha} \psi_{3,0}{ }^{2 / 3-\alpha_{3}} \leq C_{\alpha}^{\prime} t^{k / 3}\langle\xi\rangle^{1 / 6} \psi_{3,0}{ }^{-\alpha_{3}} \cdot\left|\sigma_{i s}-\sigma_{i j}\right| \\
& \leq C_{\alpha}^{\prime}\left\langle t^{e}{ }_{h} \xi\right\rangle^{1 / 6} \psi_{3,0}{ }^{-\alpha_{3}} \cdot\left|\sigma_{i s}-\sigma_{i j}\right| .
\end{aligned}
$$

(8.67) (8.70) and Leibniz rule show (8.66).

The fact that $\varphi_{i}^{*}=1$ on supp $\varphi_{i}$ and Lagrange's interpolation formula show

$$
\begin{aligned}
& L \varphi_{i 2}=b_{121}\left(L-\sigma_{i 2}\right) \varphi_{i 2}+b_{212}\left(L-\sigma_{i 1}\right) \psi_{i 2}, \\
& L^{2} \varphi_{i 2}=\sum_{k, j, s: \text { distinct }} b_{k s k} b_{k j k}\left(L-\sigma_{i s}\right)\left(L-\sigma_{i j}\right) \varphi_{i 2},
\end{aligned}
$$

where we adopt the convention (3.16) on $L$ and $\varphi_{i 2}$ is defined by (7.15). (8.66) implies

$$
b_{s j k} \in \Sigma^{0} .
$$


We set

$$
B_{s j k}=O p^{f} b_{s j k} .
$$

Now we recall the definition (7.17) of $\Phi_{i N}, \Phi_{0 l N}$. Using for $\Phi=\Phi_{i 2}$ identities that

$$
\begin{aligned}
& L_{i m} \Phi=\left[L_{i m}, \Phi\right]+\Phi L_{i m}, \\
& L_{i l} L_{i j} \Phi=\left[L_{i l},\left[L_{i j}, \Phi\right]\right]+\left[L_{i j}, \Phi\right] L_{i l}+\left[L_{i l}, \Phi\right] L_{i j}+\Phi L_{i l} L_{i j}, \\
& \tilde{L}^{2} \Phi=[\tilde{L},[\tilde{L}, \Phi]]+\Phi \tilde{L}^{2}+2[\tilde{L}, \Phi] \tilde{L},
\end{aligned}
$$

and using (8.71), (8.72), Lemmas 4.18, 4.22, 6.4, and (6.24) show

$$
\begin{aligned}
& \tilde{L}_{i 2}=B_{121} L_{i 1} \Phi_{i 2}+B_{212} L_{i 2} \Phi_{i 2}+\sum_{j=1}^{2} A_{j} L_{i j}+A_{0} \\
& \text { with } A_{j} \in \mathscr{B}^{0,3} \text { for } j \neq 0, A_{0} \in \mathcal{B}^{1 / 2,1 / 2,3}, \\
& \tilde{L}^{2} \Phi_{i 2}=\sum_{k, j, s: \text { distinct }} B_{k s k} B_{k j k} L_{i s} L_{i j}+\sum_{s \neq j} B_{s j} L_{i s} L_{i j} \\
& \quad+\sum_{s=1}^{3} B_{s} L_{i s}+\tilde{B} \tilde{L}+B_{0}
\end{aligned}
$$

with $B_{s j} \in \mathscr{B}^{0,3}, B_{s} \in \mathscr{B}^{1 / 2,1 / 2,3}$ for $s \neq 0, \tilde{B} \in \mathscr{B}^{1 / 2,1 / 2,3}, B_{0} \in \mathscr{B}^{1,1,3}$.

Using (8.74), (8.75), and using Lemma 4.21-(1), (4.55) show that for $n \geq 2$,

$$
\begin{aligned}
& \sum_{k=1}^{2}\left\|\tilde{L}^{k} \Phi_{i 2}{ }^{2} u\right\|^{(3-k, n)} \leq C_{l}\left(\sum_{s \neq j} \sum_{k=1}^{2}\left\|L_{i s} L_{i j} \Phi_{i 2}{ }^{k} u\right\|^{(1, n)}\right. \\
& \quad+\sum_{s=1}^{3} \sum_{k=1}^{2}\left\|L_{i s} \Phi_{i 2}{ }^{k} u\right\|^{(2, n)}+\left\|\tilde{L} \Phi_{i 2} u\right\|^{(2, n-1 /(10 h))}+\|u\|_{(3)}^{(u-1 /(20 h))} \\
& \quad+n^{-1}\|u\|\left(\begin{array}{l}
(n) \\
2
\end{array}\right) .
\end{aligned}
$$

If $L_{0}=D_{t}-A$ with $A=O p{ }^{f} a$ and $a \in \Sigma_{1 / 2}^{(0,-1,1)}$ satisfying (4.42), Lemmas 4. 18(3)-(i), 4.22, and the fact that $t^{-1} \mathscr{A}^{k, l, N} \subset \mathcal{A}^{k+1 / 2, l+1 / 2, N}$ when $N \geq k+1 / 2$ show

$$
\left[L_{0}, B\right] \in t^{\lambda} \mathscr{A}^{k+1 / 2, l+1 / 2, N} \quad \text { if } B \in t^{\lambda} \mathscr{A}^{k, l, N} \text { with } n \geq k+1 / 2 .
$$

So if $L_{0 j}, j=1,2$ are such operators as $L_{0}$ and $\Phi \in t^{\lambda} \mathscr{A}^{0,3}$ with $\lambda \geq 0$, using Lemma 4.21-(1), (4.55) show that for $n \geq 2+\lambda$,

$$
\begin{aligned}
& \left\|L_{01} \Phi u\right\|^{(2, n)} \leq C\left(\left\|L_{01} u\right\|^{(2, n-\lambda)}+\|u\|_{(3)}^{(n-1 /(20 h)-\lambda)}+n^{-1 / 2}\|u\|_{(2)}^{(n-\lambda)}\right), \\
& \left\|L_{01} L_{02} \Phi u\right\|^{(1, n)} \leq C^{\prime}\left(\left\|L_{01} L_{02} u\right\|^{(1, n-\lambda)}\right. \\
& \quad+\sum_{j=1}^{2}\left(\left\|L_{0 j} u\right\|^{(2, n-\lambda-1 /(20 h))}+n^{-1 / 2}\left\|L_{0 j} u\right\|^{(2, n-\lambda)}\right) \\
& \left.\quad+\|u\|_{(3)}^{(n-1) /(10 h)-\lambda)}+n^{-1}\|u\|_{(1)}^{(n-\lambda)}\right) .
\end{aligned}
$$

Applying (8.77) to each term with $k=2$ of summations on the right and to the third term, and using Lemma 4.21-(2) we obtain 


$$
\begin{aligned}
& \sum_{k=1}^{2}\left\|\tilde{L}^{k} \Phi_{i 2}{ }^{2} u\right\|^{(3-k, n)} \leq C_{2}\left(\sum_{s \neq j}\left\|L_{i s} L_{i j} \Phi_{i 2} u\right\|^{(1, n)}\right. \\
& \left.\quad+\sum_{s=1}^{3}\left\|L_{i s} \Phi_{i 2} u\right\|^{(2, n)}+\|\tilde{L} u\|^{(2, n-1 /(20 h))}+\|u\|_{(3)}^{(n-1 /(20 h))}+n^{-1}\|u\|_{\left(\begin{array}{l}
(n) \\
(2)
\end{array}\right)}\right) .
\end{aligned}
$$

Similarly we have that for $n \geq 2$,

$$
\begin{aligned}
& \sum_{k=1}^{3}\left\|\tilde{L}^{k} \Phi_{0 l 2}^{2} u\right\|^{(3-k, n)} \leq C_{3}\left(\sum_{k=1}^{2}\left\|\tilde{L}^{k} \Phi_{0 l 2} u\right\|^{(3-k, n)}+\|u\| \|_{(3)}^{(n-1 /(20 h))}\right. \\
& \left.\quad+n^{-1 / 2}\|u\|_{(2)}^{(n)}\right)
\end{aligned}
$$

and setting $\Phi_{i}=\Phi_{i 2}{ }^{2}-O p^{f}\left(\varphi_{i 2}{ }^{2}\right), \Phi_{0 l}=\Phi_{0 l 2}{ }^{2}-O p^{f}\left(\varphi_{0 l 2}{ }^{2}\right)$, and noting $\Phi_{i}, \Phi_{0 l} \in$ $t^{2 \mathrm{k} / 3} \mathcal{A}^{0,3}$ from (3.20) and Lemma 6.26 we have that

$$
\begin{aligned}
& \sum_{k=1}^{2}\left(\left\|\tilde{L}^{k} \Phi_{i} u\right\|^{(3-k, n)}+\left\|\tilde{L}^{k} \Phi_{0 l} u\right\|^{(3-k, n)}\right) \leq C_{4}\left(\sum_{k=1}^{2}\left\|\tilde{L}^{k} u\right\|^{(3-k, n-2 \kappa / 3)}\right. \\
& \left.\quad+\|u\|_{(3)}^{(n-2 \kappa / 3)}\right), n \geq 3
\end{aligned}
$$

Note that by Lemma 4.21, $\left\|\Phi_{i} u\right\|_{(6)}^{(n)}+\left\|\Phi_{0 l} u\right\|_{(6)}^{(n)} \leq C_{5}\|u\|_{(6)}^{(n-2 \kappa / 3)}$ for $n \geq 2$. Now using (8.78) (8.80), (4.55), Lemmas 4.21-(1), 7.1-(3) and (4) show that there exists $n_{1} \in \boldsymbol{N}$ and $T_{1} \in\left(0, T_{0}\right)$ such that for $T \in\left(0, T_{1}\right), n \geq n_{1}$,

$$
\begin{aligned}
& \|u\|\left\|_{(6)}^{(n)}+\sum_{k=1}^{2}\right\| \tilde{L}^{k} u \|^{(3-k, n)} \leq C_{6}\left(\sum_{i \in I}\left\|\Phi_{i 2} u\right\|_{(6)}^{(n)}+\sum_{l=1}^{2}\left\|\Phi_{0 l 2} u\right\|_{(6)}^{(n)}\right. \\
& +\sum_{i \neq j}\left\|L_{i s} L_{i j} \Phi_{i 2} u\right\|^{(1, n)}+\sum_{s=1}^{3}\left\|L_{i s} \Phi_{i 2} u\right\|^{(2, n)} \\
& \left.+\sum_{l=1}^{2} \sum_{k=1}^{2}\left\|\tilde{L}^{k} \Phi_{0 l 2} u\right\|^{(3-k, n)}\right) \leq C_{7}\left(\sum_{i \in I} M_{i, n}\left(\Phi_{i 2} u\right)+\sum_{l=1}^{2} M_{0, n}\left(\Phi_{0 l 2} u\right)\right)
\end{aligned}
$$

where we use that $\tilde{L}=L_{0, i}$ for any $i$ from the assumption (3.13)-(3). As in the proof of Proposition 8.1 we may assume that $Q=p_{3}(t, x, 1, \zeta(t, x))^{-1} O p{ }^{f} q$. Let $\Phi \in \Sigma_{1, \varphi_{i}}$ and $\pi=\prod_{j=1}^{3}\left(\tau-\tilde{\mu} f \xi-{ }^{f} \tilde{\sigma}_{i j}\right)$ where $\tilde{\mu}(t, x)=\mu(t, x) /(1-(\zeta \cdot \mu)(t, x))$ with the convention (3.16), and note that $p_{3}(t, x, 1, \zeta(t, x))^{-1}{ }^{f} q=\pi$ on supp $\varphi_{i}$. From Lemmas 6.9, 6.12

$$
\begin{aligned}
& \qquad p \pi \cdot \Phi-Q \cdot \Phi \in \mathcal{C}_{0}, \\
& {\left[Q_{3}, \Phi\right] \in \mathcal{C}+\mathcal{C}_{0} \text { with } \mathcal{C}=\mathcal{C}\left(L_{1}=L_{2}=L_{3}=\tilde{L}\right),} \\
& {\left[Q_{2}, \Phi\right] \in \mathcal{C}_{0},}
\end{aligned}
$$

where $Q_{j}=p_{3}(t, x, 1, \zeta(t, x))^{-1} O p{ }^{f} q_{j}$ with the homogeneous part $q_{j}$ of degree $j$ in $(\tau, \xi)$ of $q$.

Using (8.82), (8.83), Lemmas 7.3-(1), 7.4-(1), 4.21 one can easily deduce that for small $T$ and $n \geq 2$,

$$
M_{i, n}(\Phi u) \leq C_{8}\left(\|\left. Q u\right|^{(n)}+\sum_{k=1}^{2}\left(\left\|\Lambda_{(3-k) / 2} \widetilde{L}^{k} u\right\|^{(n+((3-k) / 2)(1-(1 / 10 h))}\right.\right.
$$




$$
\left.\left.+\left\|\tilde{L}^{k} u\right\|^{(n+3-k)}\right)+\|u\|_{(4)}^{(n-(1 / 20 h))}\right) .
$$

Next we let $\Phi_{0} \in \Sigma_{1, \varphi_{0 l}}$. In case (3) we have $g_{l}=f^{1 / 2} a_{0 l} \xi^{2}$ so that

$$
f^{1 / 2} p_{2}=g_{l}+f^{1 / 2} \sum_{j=1}^{2} a_{j l} \xi^{2-j} L^{j}
$$

So from Lemma 6.38,

$$
\Phi_{0} \cdot Q_{2}=\sum_{k=0}^{2} A_{k} \tilde{L}^{k} \text { with } A_{k} \in \mathcal{A}^{(3-k) / 2,3} \text { for } k=1,2, A_{0} \in t^{-\kappa} \mathcal{A}^{3 / 2,3} \text {. }
$$

Using this and (8.83) which holds for $\Phi \in \in^{f} \Sigma_{1 / 2}^{0}$ we obtain similarly as (8.84) that for small $T$ and $n \geq 2$,

$$
\begin{aligned}
& M_{0, n}\left(\Phi_{0} u\right) \leq C_{9}\left(\|Q u\|^{(n)}+\sum_{k=1}^{2}\left(\left\|\Lambda_{(3-k) / 2} \tilde{L}^{k} u\right\|^{(n+((3-k) / 2)(1-(1 / 10 h))}\right.\right. \\
& \left.\left.\quad+\left\|\tilde{L}^{k} u\right\|^{(n+3-k)}\right)+\|u\|_{(4)}^{(n-1 /(20 h))}\right)
\end{aligned}
$$

Now the rest of proof is straightforward by using (8.81), (8.84), (8.85), and Lemma 7.9. We omit the details. Propositions 8.1, 8.6, 8.8 mean (2.13), (2.14) respectively in cases (1), (2), (3).

\section{§9. Proof of Proposition 2.3}

We just have to (2.10) for proof of Proposition 2.3, because the standard argument gives the desired concluion. When $m=1$, or when $m=2$ and $k_{2}=1$, (2.13) contains (2.10). And the remaining cases are contained in the following Proposotion.

Proposition 9.1. Suppose the assumptions and notations in Proposition 2.3. Suppose that in (2.6), the number of paires of $(i, j)$ with $j \neq 0$ is greater than or equal to 2. Set for $s=0, \cdots, 2 m$ and $n \geq 1$,

$$
\|u\|^{[s, n]}=\sum_{i+j \leq 2 s} n^{m-(3 / 2)-(i / 2)-j}\left\|\Lambda_{i / 2} D_{t}^{j} u\right\|^{(n+m-(j / 2)-j)}, u \in \mathcal{S}_{T} .
$$

Then, there exists $C>0, n_{0} \geq 1$, and $T \in\left(0, T_{0}\right)$ such that for $T \in\left(0, T_{0}\right), n \geq n_{0}$, and $u \in \mathcal{S}_{T}$

$$
\|u\|^{[2 m, n]} \leq C\|Q u\|^{(n)} .
$$

Proof. We note that $m \geq 2$ from the assumption. In view of (2.7) the partial fraction decomposition, or rather the theory of resultants shows that one can write

$$
p_{m}+f^{1 / 2} p_{m-1}^{\prime}=\prod_{i} \prod_{j \neq 0}\left(p_{i j}+f^{1 / 2} p_{i j}^{\prime}\right)+q_{m-2}
$$


with the notation $p_{m-1}^{\prime}(t, x, \tau, \xi)=p_{m-1}(t, x, \tau,-\zeta(t, x) \tau+\xi)$ where $p_{i j,}^{\prime}, q_{m-2}$ are in $C^{\infty}\left(\boldsymbol{R}_{T_{0}}^{4}\right)$ being polynomials in $(\tau, \xi)$ with their coeficients in $B_{T_{0}}$ respectively of degrees $i-1, m-2$. We may neglect perturbations of terms of order $\leq m-2$ in $\tilde{Q}$ for proof of (9.1) in view of (9.1) and Lemma 4.21-(1) so that we may assume

$$
\tilde{Q}=p_{m}(t, x, 1, \zeta(t, x))^{-1}\left(p_{m}+f^{1 / 2} p_{m-1}-q_{m-2}\right)(t, x, \tau, \zeta(t, x) \tau+\xi) .
$$

Set for $j \neq 0$,

$$
\begin{aligned}
& \tilde{Q}_{i j}=p_{i j}(t, x, 1, \zeta(t, x))^{-1}\left(p_{i j}+f^{1 / 2} p_{i j}^{\prime}\right)(t, x, \tau, \zeta(t, x) \tau+\xi), \\
& Q_{i j}={ }^{f} \tilde{Q}_{i j}, \quad Q^{i j}=\Pi_{(1, s) \neq(i, j)} Q_{l s} .
\end{aligned}
$$

Then

$$
Q(t, x, \tau, \xi)=\Pi_{i, j} Q_{i j}(t, x, \tau, \xi)
$$

Similarly as above we can write for each $1 \leq k \leq m$,

$$
\xi^{k} \tau^{m-k}=\sum_{i, j} r_{i j k} \tilde{Q}^{i j}+r_{k}
$$

where $r_{i j k}, r_{k}$ are in $C^{\infty}\left(\boldsymbol{R}_{T_{0}}^{4}\right)$ which are polynomials in $(\tau, \xi)$ with coeficients in $B_{T_{0}}$ such that deg $r_{i j k}=i$, degree in $\tau$ of $r_{i j k}=i-1$, deg $r_{k}=m-1$, degree in $\tau$ of $r_{k}=m-2$.

Using (9.4), (2.13), Lemmas 7.1-(2), 4.21-(1), and the inequality $\left\|D_{\imath}^{m} u\right\|^{(n)} \leq$ $C\left(\|Q u\|^{(n)}+\sum_{k=1}^{m} \sum_{l=0}^{m-k}\left\|f^{k} D_{x}^{k} D_{l}^{l} u\right\|^{(n)}\right)$, one finds that for small $T$ and large $n$,

$$
\begin{aligned}
& \|u\|^{[2 m, n]}-C_{1} n^{-3 / 2}\|Q u\|^{(n)} \leq C_{2}\left(n^{-3 / 2} \sum_{k=1}^{m}\left\|f^{k} D_{x}^{k} D_{\xi}^{m-k} u\right\|^{(n)}+n^{-1}\|u\|^{[2 m-2, n-1]}\right) \\
& \quad \leq C_{3}\left(\sum_{i, j}\left\|Q_{i j} \circ Q^{i j}(t, x, D) u\right\|^{(n)}+n^{-1}\|u\|^{[2 m-2, n-1]}\right)
\end{aligned}
$$

Let $e_{1}=(1,0), e_{2}=(0,1) \in \boldsymbol{R}^{2}$. Then, symbolic calculus shows that by extending Definition 6.21 to $q$ of the form in it with $a_{30}=$ cosnt.,

$$
\begin{aligned}
& \left(\tilde{Q}_{i j}\right)_{0, e_{l}}=\sum_{k=0}^{i-1} a_{k l} \tau^{k} \text { with } \\
& a_{k 1} \in t^{1 / 2-1 /(20 h) f} \Sigma^{(i-k-1 / 2)(0,-2,0)}, a_{k 2} \in^{f} \sum^{(i-k-1 / 2)(0,-2,0)} ; \\
& \left(\tilde{Q}_{i j}\right)_{e_{1}, 0^{\circ}}\left(\tilde{Q}_{i^{\prime} j^{\prime}}\right)_{0, e_{1}}-\left(\tilde{Q}_{i j}\right)_{e_{1}, 0} \cdot\left(\tilde{Q}_{i^{\prime} j^{\prime}}\right)_{0, e_{1}}=\sum_{k=0}^{i+i^{\prime}-2} a_{k} \tau^{k} \\
& \text { with } a_{k} \in \sum_{0<l \leq i+b^{\prime}-k-2} t^{-1+1 / 2-1 /(20 h)} B^{i+i^{\prime}-k-l-1,1 / 2, m} \\
& +t^{-1 / 2-1 /(20 h)} B^{i+i^{\prime}-k-2, m} \text {; } \\
& \left(\tilde{Q}_{i j}\right)_{e_{2}, 0^{\circ}} \circ\left(\tilde{Q_{i^{\prime} j^{\prime}}}\right)_{0, e_{2}}-\left(\tilde{Q_{i j}}\right)_{e_{2}, 0} \cdot\left(\tilde{Q_{i^{\prime} j^{\prime}}}\right)_{0, e_{2}}=\sum_{k=0}^{i+i^{\prime}-2} b_{k} \tau^{k}
\end{aligned}
$$


with $b_{k} \in B^{i+i^{\prime}-k-2, m}$;

$$
\begin{aligned}
& {\left[O p Q_{i^{\prime} j^{\prime}}, O p\left(\tilde{Q}_{i j}\right)_{0, e_{l}}\right]=\sum_{k=0}^{i+i^{\prime}-2} A_{k l} D_{t}^{k}} \\
& \text { with } A_{k l} \in \sum_{0<s \leq i+i^{\prime}-k-2} t^{-s+1 / 2-1 /(20 h)} \mathscr{B}^{i+i^{\prime}-k-s-1 / 2, m} \\
& \quad+t^{1 / 2-1 /(20 h)} \mathscr{B}^{i+i^{\prime}-3 / 2-k, m}, A_{k 2} \in \mathscr{B}^{i+i^{\prime}-k-3 / 2, m}
\end{aligned}
$$

From (9.3), (9.7), (9.8), and the equalities

$$
\begin{aligned}
& \left(\tilde{Q}_{i j}\right)_{e_{1}, 0} \cdot\left(\tilde{Q}_{i^{\prime} j^{\prime}}\right)_{0, e_{1}}=\partial_{\tau} Q_{i j} \cdot \partial_{t} Q_{i^{\prime} j^{\prime}}, \\
& \left(\tilde{Q}_{i j}\right)_{e_{2}, 0} \cdot\left(\tilde{Q}_{i^{\prime} j^{\prime}}\right)_{0, e_{2}}={ }^{f}\left(\partial_{\xi} \tilde{Q}_{i j}\right) f D_{x} Q_{i^{\prime} j^{\prime}} \text {, } \\
& \left(\tilde{Q}_{i j}\right)_{e_{l}, 0^{\circ}}\left(\tilde{Q}_{i^{\prime} j^{\prime}}\right)_{0, e_{l}} \circ Q^{i j, i^{\prime} j^{\prime}}-\left(\left(\tilde{Q}_{i j}\right)_{e_{l}, 0} \cdot\left(\tilde{Q}_{i^{\prime} j^{\prime}}\right)_{0, e_{l}}\right) \cdot Q^{i j, i^{\prime} j^{\prime}} \\
& =\left(\left(\tilde{Q}_{i j}\right)_{e_{l}, 0^{\circ}}\left(\tilde{Q}_{i^{\prime} j^{\prime}}\right)_{0, e_{l}}-\left(\tilde{Q}_{i j}\right)_{e_{l}, 0} \cdot\left(\tilde{Q}_{i^{\prime} j^{\prime}}\right)_{0, e_{l}}\right) \circ Q^{i j, i^{\prime} j^{\prime}} \\
& +\left(\left(\tilde{Q}_{i j}\right)_{e_{l}, 0} \cdot\left(\tilde{Q}_{i j}\right)_{0, e_{l}}\right) \circ Q^{i j, i^{\prime} j^{\prime}}-\left(\left(\tilde{Q}_{i j}\right)_{e_{l}, 0} \cdot\left(\tilde{Q}_{i^{\prime} j^{\prime}}\right)_{0, e_{l}}\right) \cdot Q^{i j, i^{\prime} j^{\prime}} \\
& \text { where } Q^{i j, i^{\prime} j^{\prime}}=\Pi_{(l, s) \neq(i, j),\left(i^{\prime}, j^{\prime}\right)} Q_{l s} \text {, }
\end{aligned}
$$

we see that

$$
\begin{aligned}
& Q_{i j} \circ Q^{i j}=Q+\sum_{l=1}^{2} \sum_{(i, j) \neq\left(i^{\prime}, j^{\prime}\right)}\left(\tilde{Q}_{i j}\right)_{e_{l}, 0^{\circ}}\left(\tilde{Q}_{i^{\prime} j^{\prime}}\right)_{0, e_{l}} \circ Q^{i j, i^{\prime} j^{\prime}}+\sum_{k \leq m-2} \tilde{a}_{k} \tau^{k} \\
& \quad \text { with } \tilde{a}_{k} \in \sum_{0<s \leq m-2}\left(t^{-s+1 / 2-1 /(20 h)} B^{m-k-s-1,1 / 2, m}+t^{-1 / 2-1 /(20 h)} B^{m-k-2, m}\right) .
\end{aligned}
$$

This and (2.13) imply that for small $T$ and large $n$,

$$
\begin{aligned}
& \sum_{i, j}\left\|Q_{i j} \circ Q^{i j}(t, x, D) u\right\|^{(n)} \\
& \leq C_{4}\left(\|Q u\|^{(n)}+\sum_{l=1}^{2} \sum_{(i, j) \neq\left(i^{\prime}, j^{\prime}\right)}\left\|Q_{i j^{\prime}} \circ\left(\tilde{Q}_{i^{\prime} j^{\prime}}\right)_{0, e_{l}} \circ Q^{i j, i^{\prime} j^{\prime}}(t, x, D) u\right\|^{(n)}\right. \\
& \left.\quad+\|u\|^{[2 m-3, n-1 / 2]}\right) .
\end{aligned}
$$

By (9.9), (9.6), (2.14), a term in the summation on the right is estimated for small $T$ and large $n$ by

$$
\begin{aligned}
& \left\|\left(\tilde{Q}_{i^{\prime} j^{\prime}}\right)_{0, e_{l}} \circ Q_{i j^{\circ}} \circ Q^{i j, i^{\prime} j^{\prime}}(t, x, D) u\right\|^{(n)}+\|u\|^{[2 m-3, n-1 / 2]} \\
& \leq C_{5}\left(\left\|\left(\tilde{Q}_{i^{\prime} j^{\prime}}\right)_{0, e_{l}} \circ Q^{i^{\prime}, j^{\prime}}(t, x, D) u\right\|^{(n)}+\|u\|^{[2 m-3, n-1 / 2]}\right) \\
& \leq C_{6}\left(\left\|Q_{i^{\prime} j^{\prime}} \circ Q^{i^{\prime} j^{\prime}}(t, x, D) u\right\|^{(n-1) /(20 h)}\right. \\
& \left.\quad+n^{-1 / 2}\left\|Q_{i^{\prime} j^{\prime}} \circ Q^{i^{\prime} j^{\prime}}(t, x, D) u\right\|^{(n)}+\left.\|u\|\right|^{[2 m-3, n-1 / 2]}\right) .
\end{aligned}
$$

Therefore we obtain that for small $T$ and large $n$,

$$
\sum_{i, j}\left\|Q_{i j} \circ Q^{i j}(t, x, D) u\right\|^{(n)} \leq C_{7}\left(\|Q u\|^{(n)}+\|u\|^{[2 m-3, n-1 / 2]}\right) .
$$

The desired conclusion follows from (9.5) and (9.10).

Q.E.D. 


\section{§ 10. Appendix. Proof of Lemma 4.7}

Our proof is parallel to that of Lemma 2 in [1]. So we make our discription brief. We denote the set of all $b$ as in the statement satisfying (4.18) for $(\Phi, \varphi) \in B$ by $\mathcal{U}^{\Phi, \varphi}$, and we set $\mathcal{U}=\bigcup_{(\Phi, \varphi) \in B} \mathcal{U}^{\Phi, \varphi}$. We denote $a(x, \xi)$ as in the above defined for $b \in \mathcal{U}$ by $I(b)(x, \xi)$. The important fact is that constants in proof are uniform on $B$ and $\mathcal{U}$, but we omit to mention this fact for simplicity.

First we assume that the lemma has proved for $b \in \mathcal{V}$ compactly supported in $(y, \eta)$ for each fixed $(x, \xi)$. Let $b \in \mathcal{Q}^{\Phi, \varphi}$ and set $b_{m}=\chi_{m} b$ for $m \in \boldsymbol{N}$ where $x_{m}(x, y, \xi, \eta)=\chi(y / m, \eta / m)$ with $\chi \in C_{0}^{\infty}\left(\boldsymbol{R}^{2 n}\right)$ with $\chi(0)=1$. Then

$$
\left|D_{y}^{\alpha} D_{\eta}^{\beta} b_{m}(. x, y, \xi, \eta)\right| \leq C_{\alpha \beta}|b|_{|\alpha|+|b|}^{\Phi, \varphi} \cdot \sum_{i \leq \mid \beta} \sup _{Q \in \mathbb{K}} \Phi^{-p}(Q) \cdot a_{|\alpha|,|\beta|-p}^{\Phi, \varphi}
$$

with $C_{\alpha \beta}$ independent of $m$, and $I\left(b_{m}\right)$ converges to $I(b)$ at any point. So the lemma for general $b$ follows from the above assumed special case by replacing $k$ by $k+1$ and $\left(M_{1}, \cdots, M_{k+l}, m_{1}, \cdots, m_{k+l}\right)$ by $\left(0, M_{1}, \cdots, M_{k+l}, 0, m_{1}, \cdots, m_{k+l}\right)$. Thus, we just have to prove (4.19) with $C^{\prime}, L$ independent of $b \in \mathcal{U}^{\Phi, \varphi}$ compactly uspported in $(y, \eta)$ for each fixed $(x, \xi)$, and of $(\Phi, \varphi)$.

We note that $I(b)=\iint e^{-i(y-x) \cdot(\eta-\xi)} b(x, y, \xi, \eta) d y d \eta$ then. Let $x_{1} \in C^{\infty}(\boldsymbol{R})$ with $x_{1}(z)=1$ when $|z| \leq 1 / 3, \quad x_{1}(z)=0$ when $|z| \leq 1 / 2$, and write $b=b_{1}+b_{2}$ where $b_{1}(x, y, \xi, \eta)=b(x, y, \xi, \eta) \chi_{1}(|\eta-\xi| /(1+|\xi|))$. Then $b_{i} \in^{\Phi, \varphi}$ and $\left|b_{i}\right|_{j}^{\phi, \varphi} \leq C_{1 j}|b|_{j}^{\Phi, \varphi}$ so that $I(b)=I\left(b_{1}\right)+I\left(b_{2}\right)$. We have

$$
\begin{array}{ll}
b_{1}=0 & \text { when } \quad|\xi-\eta| \geq(1+|\xi|) / 2, \\
b_{2}=0 & \text { when } \quad|\xi-\eta| \leq(1+|\xi|) / 3 .
\end{array}
$$

The integration by parts gives that $I\left(b_{2}\right)=I\left(b_{2}^{\prime}\right)$ with $b_{2}^{\prime} \in \mathcal{Q}^{\Phi, \varphi}$ given by $b_{2}^{\prime}(x, y, \xi, \eta)=|\eta-\xi|^{-2 N}\left(-\Delta_{y}\right)^{N}\left[\left(1+|x-y|^{2 P}\right)^{-1}\left(1+\left(-\Delta_{\eta}\right)^{P}\right) b_{2}\right]$. Taking $P>$

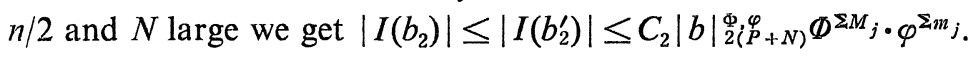

Next choose $\chi_{2} \in C^{\infty}(\boldsymbol{R})$ with $\chi_{2}(z)=1$ when $|z| \leq 1 / 9, \chi_{2}(z)=0$ when $|z| \geq 1 / 2$, and write $b_{1}=b_{11}+b_{12}$ where $b_{11}=b_{1}(x, y, \xi, \eta) \chi_{2}\left(c^{-2}\left(\varphi^{-2}(x, \xi)|y-x|^{2}+\right.\right.$ $\left.\left.\Phi^{-2}(x, \xi)|\xi-\eta|^{2}\right)\right)$. Again $b_{1 i} \in \mathcal{Q}^{\Phi, \varphi}$ and $\left|b_{1 i}\right|_{j}^{\Phi, \varphi} \leq C_{3 j}|b|_{j}^{\Phi, \varphi}$ so that $I\left(b_{2}\right)=$ $I\left(b_{21}\right)+I\left(b_{22}\right)$. We have

$$
\begin{array}{lll}
b_{11}=0 & \text { when } & \varphi^{-1}(x, \xi)|y-x|+\Phi^{-1}(x, \xi)|\eta-\xi| \geq c / 2, \\
b_{12}=0 & \text { when } & \varphi^{-1}(x, \xi)|y-x|+\Phi^{-1}(x, \xi)|\eta-\xi| \leq c / 3 .
\end{array}
$$

Setting $\Phi_{0}=\Phi(x, \xi), \varphi_{0}=\varphi(x, \xi)$ we have that when $(x, y, \xi, \eta) \in \operatorname{supp} b_{11}$, $C^{-1} \leq\left|\Phi(Q) / \Phi_{0}\right| \leq C, C^{-1} \leq\left|\varphi(Q) / \varphi_{0}\right| \leq C$ for $Q \in K$. Then the integration by parts gives $I\left(b_{11}\right)=I\left(b_{11}^{\prime}\right)$ with $b_{11}^{\prime} \in \mathcal{Q}$ given by $b_{11}^{\prime}=\left(1+\Phi_{0}^{2 P}|x-y|^{2 P}\right)^{-1} \times$ 
$\times\left(1+\Phi_{0}^{2 P}\left(-\Delta_{y}\right)^{P}\right) b_{11}$, and $\left|I\left(b_{11}\right)\right|=\left|I\left(b_{11}^{\prime}\right)\right| \leq C_{4}|b|_{2 P}^{\Phi, \varphi} \Phi_{0}^{\Sigma_{1} M_{j}} \cdot \varphi_{0}^{\Sigma_{m} m_{j}}$ by taking $P>n / 2$. Finally we consider $b_{12}$. As in the same way of [1] setting $\Phi_{0}=$ $\Phi(x, \xi), \varphi_{0}=\varphi(x, \xi)$, and $R=\Phi_{0} / \varphi_{0}$ we have that for $(x, y, \xi, \eta) \in \operatorname{supp} b_{12}$ and $Q \in K, C_{0}^{-1} R \leq \Phi(Q) / \varphi(Q) \leq C_{0} R, \Phi_{0} / \Phi(Q) \leq\left(C_{0} c^{-1}\right)^{1 / 2}\left(\Phi_{0} \varphi_{0}\right)^{1 / 2}, \Phi(Q) \leq$ $2 \cdot \max \left(c^{-1} C_{0}, 3 C c^{-1}\right)(|\eta-\xi|+R|y-x|)$. By using the first inequality the next two means respectively

$$
\varphi_{0} / \varphi(Q) \leq C_{5}\left(\Phi_{0} \varphi_{0}\right)^{1 / 2}, \quad \varphi(Q) \leq C_{6}\left(R^{-1}|\eta-\xi|+|y-x|\right) .
$$

Set $b_{21}^{\prime}(x, y, \xi, \eta)=\left(\varphi_{0}^{-2 N}\left(-\Delta_{\eta}\right)^{N}+\Phi_{0}^{-2 N}\left(-\Delta_{y}\right)\right)\left\{b_{21}(x, y, \xi, \eta) /\left(\varphi_{0}^{-2 N}|y-x|^{2 N}+\right.\right.$ $\left.\left.+\Phi_{0}^{-2 N}|\eta-\xi|^{2 N}\right)\right\}$. Then $b_{21}^{\prime} \in \mathcal{U}^{\Phi, \varphi}$ and $I\left(b_{21}\right)=I\left(b_{21}^{\prime}\right)$. Leibniz rule shows that

$$
\begin{aligned}
& \left|b_{21}^{\prime}(x, y, \xi, \eta)\right| \\
& \quad \leq C_{7 N} \varphi_{0}^{-2 N} \Phi_{0}^{-2 N}\left\{\frac{\Sigma_{|\beta| \leq 2 N}\left|D_{\eta}^{\beta} b_{21}(t, y, \xi, \eta)\right| \Phi_{0}^{|\beta|}}{\left(\varphi_{0}^{-1}|y-x|+\Phi_{0}^{-1}|\eta-\xi|\right)^{2 N+(2 N-|\beta|)}}+\right. \\
& \left.\quad+\frac{\Sigma_{|\alpha| \leq 2 N}\left|D_{y}^{\alpha} b_{21}(t, x, \xi, \eta)\right| \varphi_{0}^{\left|\alpha_{1}\right|}}{\left(\varphi_{0}^{-1}|y-x|+\Phi_{0}^{-1}|\eta-\xi|\right)^{2 N+\left(2 N-\left|\Phi_{1}\right|\right)}}\right\} \\
& \quad=C_{7 N} \varphi_{0}^{-2 N} \Phi_{0}^{-2 N}\{I+I I\} .
\end{aligned}
$$

We take $P>\sum_{j=1}^{k+1}\left(\left|M_{j}\right|+\left|m_{j}\right|\right)$ and set $\tilde{M}_{j}=M_{j}+P, \tilde{m}_{j}=m_{j}+P$. Then $\tilde{M}_{j} \geq 0$, $\tilde{m}_{j} \geq 0$. By virtue of $\Phi \varphi \geq c$ a term of the numerator in $I$ is dominated by $c^{-P(k+1)}|b|_{2 N}^{\Phi, \varphi}$ times

$$
\begin{aligned}
& \quad \sum_{r_{1}+\cdots+r_{k}=|\beta|} \Phi_{0}^{|\beta|} \prod_{j=1}^{k} \sup _{Q \in \mathbb{K}} \Phi^{\tilde{M}_{j}-r_{j}}(Q) \varphi^{\tilde{m}_{j}}(Q) \prod_{j=k+1}^{k+l} \sup _{Q \in \mathbb{K}} \Phi^{\tilde{M}_{j}}(Q) \varphi^{\tilde{m}} j(Q) \\
& \leq C_{8} \prod_{j=1}^{k+1}\left(\sup _{Q \in \mathbb{K}} \Phi^{\tilde{M}_{j}}(Q) \varphi^{\tilde{m}_{j}}(Q)\right) \sup _{Q \in \mathbb{K}}\left(\Phi_{0} / \Phi(Q)\right)^{|\beta|} \\
& \leq C_{9}\left(\Phi_{0} \varphi_{0}\right)^{N}(|\eta-\xi|+R|y-x|)^{\Sigma \tilde{M}_{j} \cdot\left(R^{-1}|\eta-\eta|+|y-x|\right)^{\Sigma \tilde{m}_{j}}}
\end{aligned}
$$

Thus $I \leq C_{10}|b|_{2 N}^{\Phi, \varphi} \Phi_{0}^{\Sigma \tilde{M}_{j}-N} \varphi_{0}^{\Sigma \tilde{m}_{j}-N}\left(\varphi_{0}^{-1}|y-x|+\Phi_{0}^{-1}|\eta-\xi|\right)^{\Sigma\left(\tilde{M}_{j}+\tilde{m}_{j}\right)-2 N} . \quad I I$ is dominated by the same expression. Therefore,

$$
\left|I\left(b_{21}\right)\right| \leq C_{11}|b|_{2 N}^{\Phi, \varphi} \cdot \Phi_{0}^{\Sigma \tilde{M}_{j}+n-N} \cdot \varphi_{0}^{\Sigma \tilde{m}_{j}+n-N} \text { if } 2 N>\sum_{i=1}^{k+1}\left(\tilde{M}_{j}+\tilde{m}_{j}\right)+n .
$$

This completes the proof.

\section{References}

[1] Beals, R. and Fefferman, C., Spatially inhomogeneous pseudo-differential operators, Comm. Pure. Appl. Math., 27 (1974), 1-27.

[2] Calderón, A.P., Uniqueness in the Cauchy problem for partial differential operators, 
Amer. J. Math., 80 (1958), 16-36.

[ 3 ] Cohen, P., The non uniqueness in the Cauchy problem, O.N.R. Techn. Report 93, Stanford, 1960.

[4] Hörmander, L., The Analysis of Linear Partial Differential Operators IV, Springer.

[5] Lerner, N., Unicité du problème pour de opérateurs elliptiques, Ann. scient. Ec. Norm. Sup. 4 serie., 17 (1984), 469-505.

[6] Matsumoto, W., Uniqueness in the Cauchy problem for partial differential equations with multiple characteristic roots, J. Math. Kyoro Univ., (1975), 479-525.

[7] Nakane, S., Uniqueness and non uniqueness in the Cauchy problem for a class of operators degenerate type, Proc. Jap. Acad., 58 (1982), 141-149.

[8] Ōkaji, T., Uniqueness in the Cauchy problem for a class of partial differetial operators degenerate on the initial surface, J. Math. Kyoto Univ., 24-3 (1984), 423-439.

[9] Ounaïres, H., Unicité du problème de Cauchy pour des operateurs différentiels à caractéristiques non régulières, These de 3e cycle. Universite Paris Sud, Orsay, 1982.

[10] Pliš, A., A smooth linear elliptic equation without any solution in a sphere, Comm. Pure. Appl. Math., 14 (1961), 599-617.

[11] Raymond, X.R., L'unicité pour les problèmes des Cauchy linéaires du premier order, L'Enseign. Math., 32 (1986), 1-55.

[12] Watanabe, K., Sur l'unicité du problème du Cahuchy pour les opérateur différentiels a caractéristiques de multiplicite constante, Tohoku Math. J. 31 (1979), 151-164.

[13] — L'unicité du prolongment des solutions de equations elliptiques dégénérées, Tohoku Math. J., 34 (1982), 239-249.

[14] Zuily, C., Uniqueness and Non Uniqueness in the Cauchy Problem, Birkhäuser.

[15] - - Unicité du problème de Cauchy pour des opérateurs elliptiques à caractéristiques hautes multiples, Comm. in P.D.E., 10(3) (1985), 219-244. 